\title{
A Heavy Flavor Tracker for STAR
}

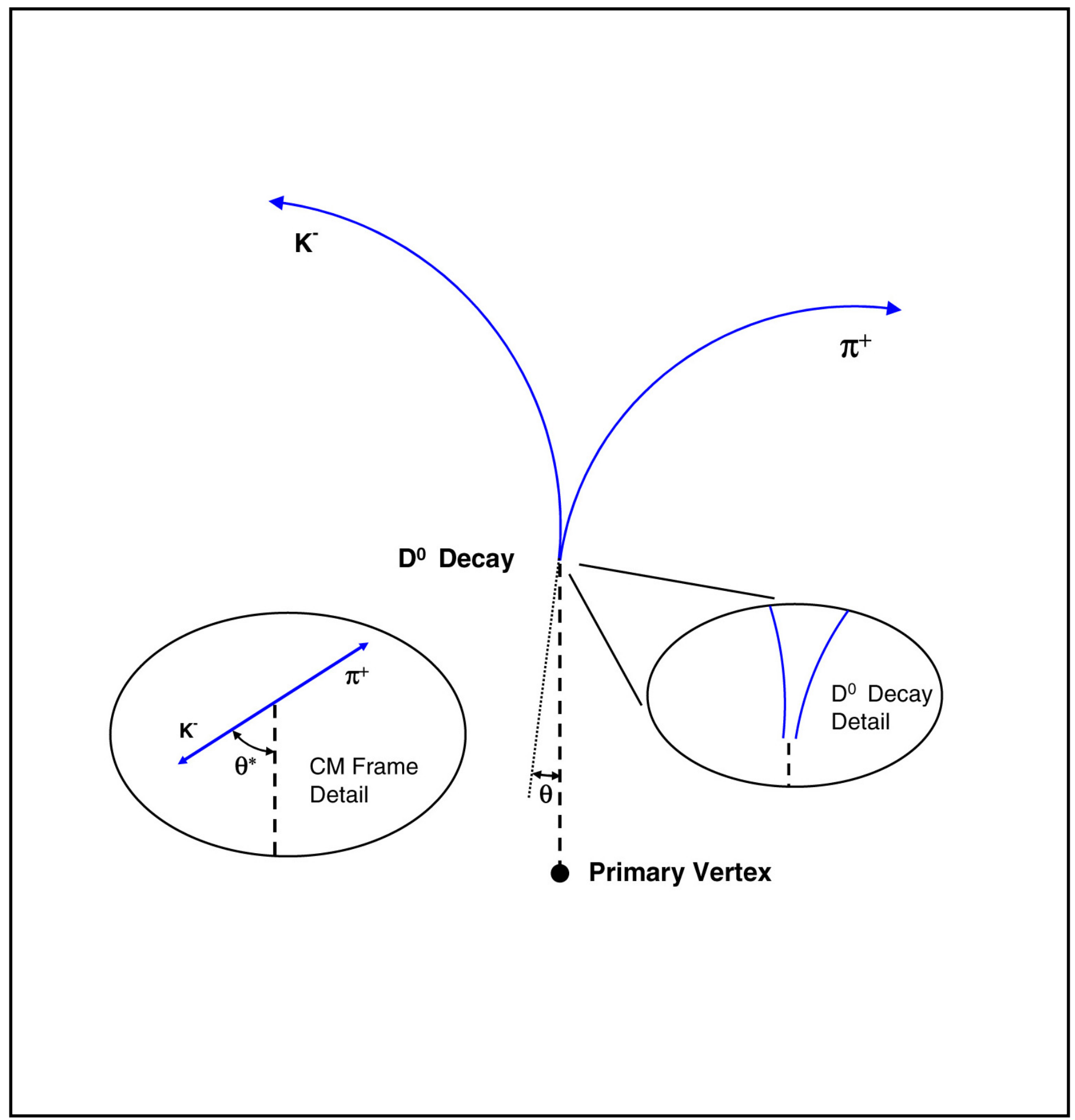


hft-06-08-2006.doc

This page intentionally left blank 


\title{
A Heavy Flavor Tracker for STAR
}

\author{
Z. Xu \\ Brookhaven National Laboratory, Upton, New York 11973 \\ Y. Chen, S. Kleinfelder, A. Koohi, S. Li \\ University of California, Irvine, California \\ H. Huang, A. Tai \\ University of California, Los Angeles, California 90095
}

V. Kushpil, M. Sumbera

Nuclear Physics Institute AS CR, 25068 Rez/Prague, Czech Republic

C. Colledani, W. Dulinski, A. Himmi, C. Hu, A. Shabetai, M. Szelezniak, I. Valin, M. Winter

Institut de Recherches Subatomique, Strasbourg, France

B. Surrow, G. Van Nieuwenhuizen

Massachusetts Institute of Technology, Cambridge, MA 02139

L. Greiner, Y. Lu, H.S. Matis, M. Oldenburg, H.G. Ritter, F. Retiere, A. Rose, L. Ruan, K. Schweda, E. Sichtermann, J.H. Thomas, H. Wieman, N. Xu, Y. Zhang

Lawrence Berkeley National Laboratory, Berkeley, California 94720

I. Kotov

Ohio State University, Columbus, Ohio 43210 
This page intentionally left blank 


\section{Contents}

1 EXECUTIVE SUMMARY ……........................................................................ 15

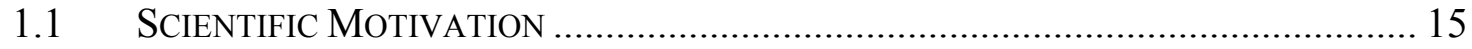

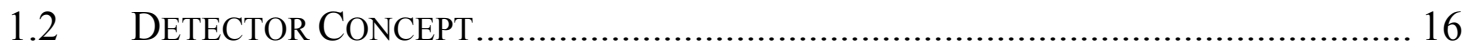

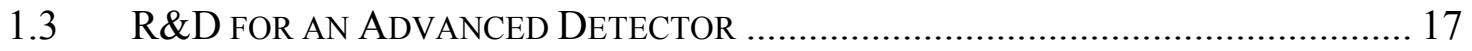

1.4 COORDINATION WITH OTHER NEW STAR DETECTORS ……….......................... 17

2 THE PHYSICS OF THE HFT ……...................................................................... 18

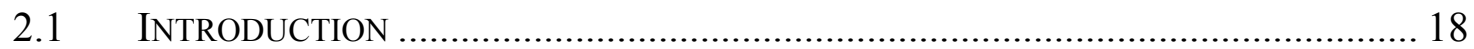

2.2 INITIAL HEAVY QUARK PRODUCTION ............................................................. 20

2.3 The NeEd For Direct Topological ReCONSTRUCtion of OPEN ChaRm ..... 23

2.4 Probing Medium Thermalization: Charm Quark RE-INTERACTIONS ....... 24

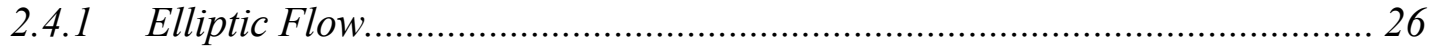

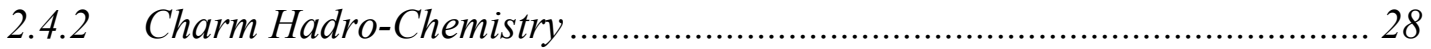

2.5 Probing THE Density of THE Medium: HeAvy QuARK ENERgy LosS .......... 30

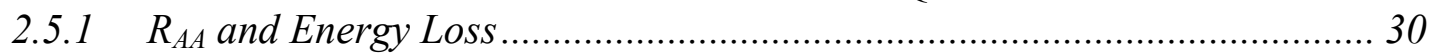

2.5.2 Charm Angular Correlations........................................................... 32

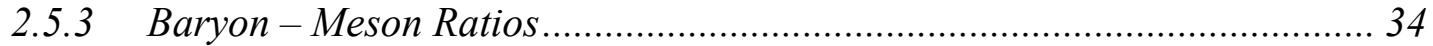

2.6 MEASURING Vector Mesons with DilePtons ............................................... 35

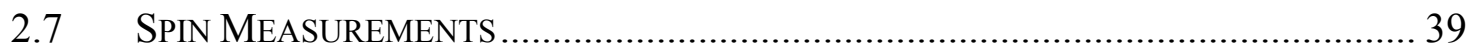

3 HFT OVERVIEW AND SIMULATION ........................................................ 40

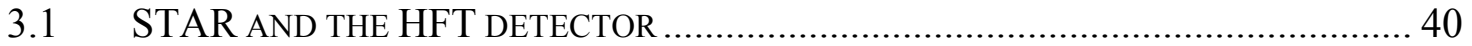

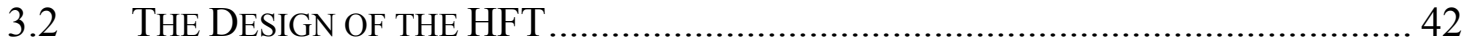

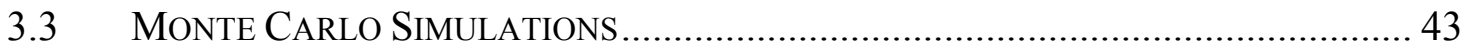

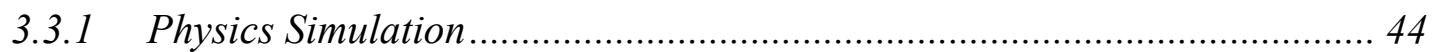

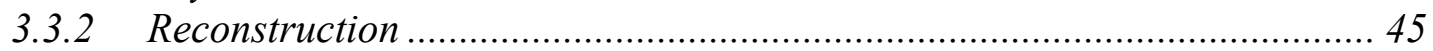

3.3.3 Primary Track Reconstruction Performance ............................................ 49

3.3.4 Analytic Resolution Calculations and Expected Performance .................... 51

3.4 OPEN CHARM RECONSTRUCTION SIMULATION ………………………............. 52

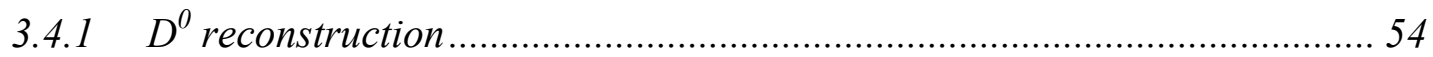

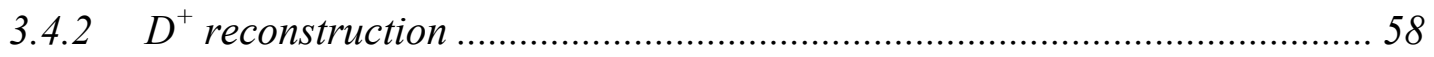

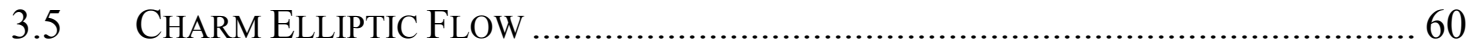

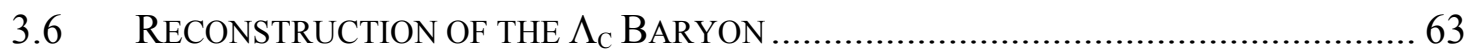

3.7 Hit DENSITY AT THE Front SURFACE OF THE HFT ……….................................. 65

3.7.1 Measured Hit Density at $6 \mathrm{~cm}$ Radius in STAR ........................................... 66

4 CMOS SENSORS .............................................................................................. 67

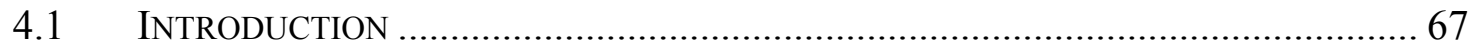

4.2 Main Features and Performance of CMOS Active Pixel Sensors ........ 70

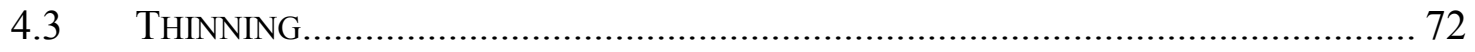

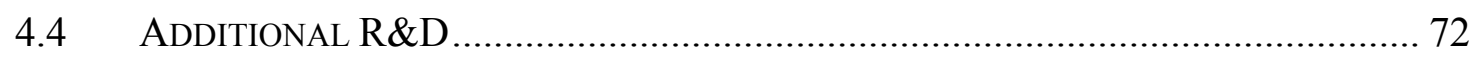

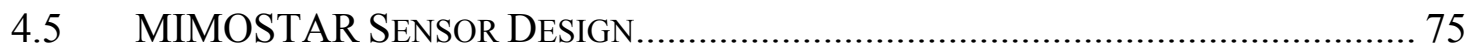

4.6 THE PATH TO A CMOS DETECTOR FOR THE HFT ……................................... 76

4.6.1 MIMOSTAR-3 - a half sized chip ........................................................ 77

4.6.2 MIMOSTAR-4 - a full sized chip ......................................................... 77 


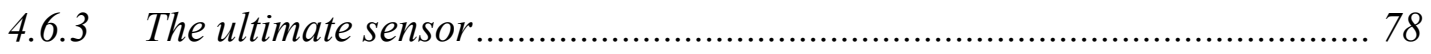

5 DATA ACQUISITION AND READOUT ..................................................... 80

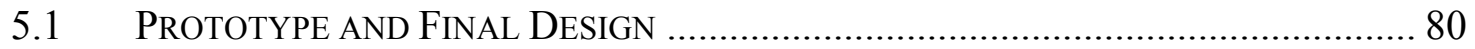

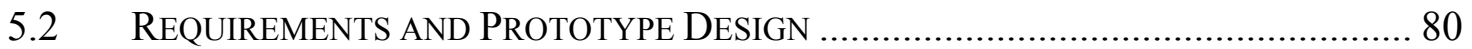

5.3 ARCHITECTURE FOR THE PROTOTYPE SYSTEM............................................... 81

5.4 DATA SYNCHRONIZATION, READOUT AND LATENCY ...................................... 85

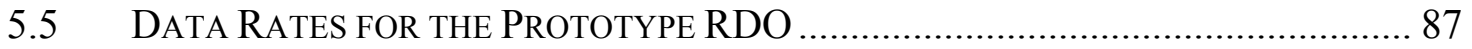

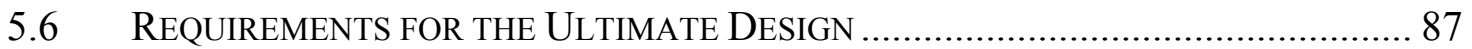

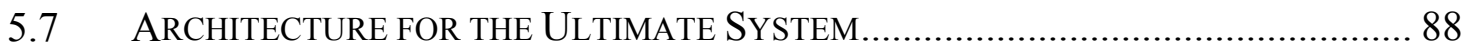

5.8 DATA RATES FOR THE Ultimate RDO ...................................................... 89

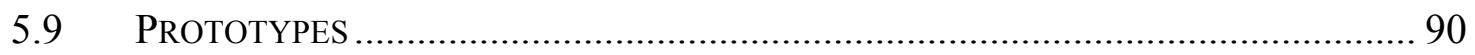

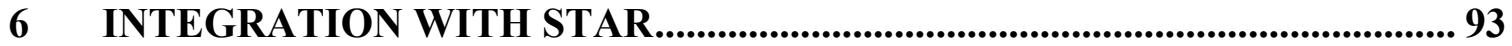

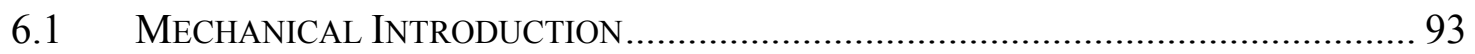

6.2 SUPPORT CARRIAGE FOR RAPID INSTALLATION AND REMOVAL ........................ 93

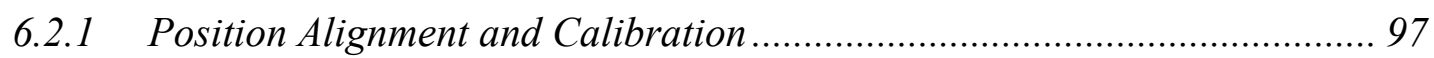

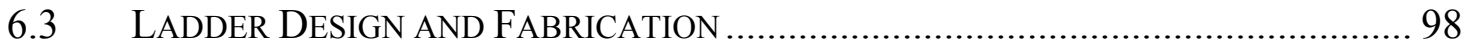

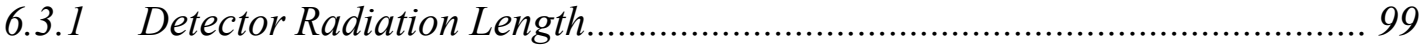

6.3.2 Expected Radiation Exposure ................................................................... 99

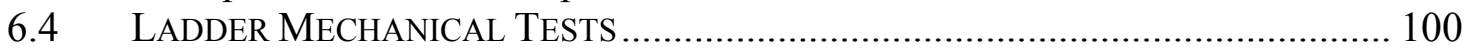

6.4.1 Load Distortion Tests........................................................................... 100

6.4.2 Thermal Distortion Tests .................................................................... 102

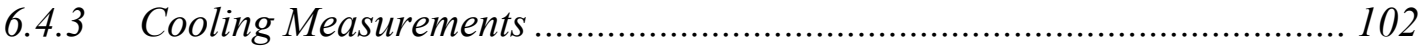

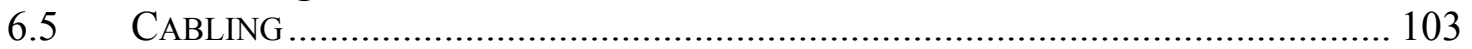

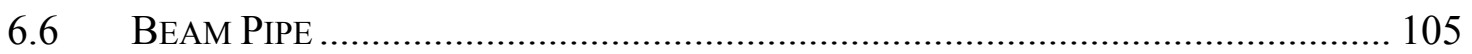

6.6.1 Minimum Beam Pipe Radius Consistent with Injected Beam ................... 106

6.6.2 Beam Pipe Radius - Vacuum Considerations ........................................ 106

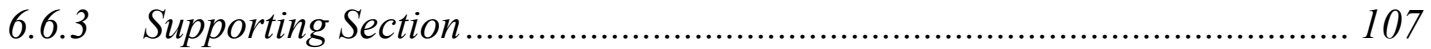

6.6.4 Central Beam Pipe Thin Window Section............................................... 108

6.6.5 RF Background from the Wake Field ............................................... 108

6.6.6 Beam Pipe Insertion and Removal....................................................... 108

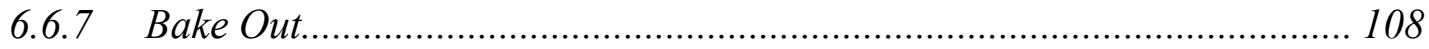

6.7 COMPATIBILITY WITH THE SSD AND OTHER CONE MOUNTED DETECTORS ....... 109

6.8 COMPATIBILITY WITH AN UPGRADED INNER Si TRACKER BARREL.................. 109

7 PRELIMINARY COST AND SCHEDULE ESTIMATES............................. 110

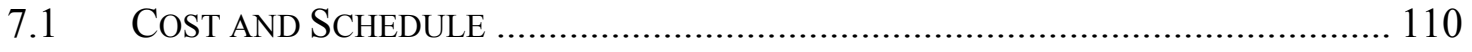

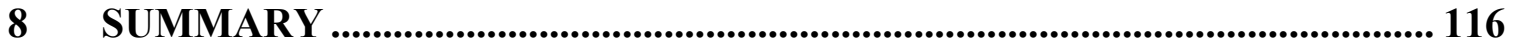

9 APPENDIX I - MIMOSTAR II SPECIFICATIONS ................................... 117

10 APPENDIX II - MIMOSTAR II USERS GUIDE...................................... 118

11 APPENDIX III - HFT PROTOTYPE READ OUT ..................................... 119

12 APPENDIX IV - PRODUCTION SCHEDULE .......................................... 127

13 APPENDIX V - COST AND SCHEDULE PRESENTATION........................ 128

14 APPENDIX VI - REPORT OF THE TECHNICAL COMMITTEE............. 129 


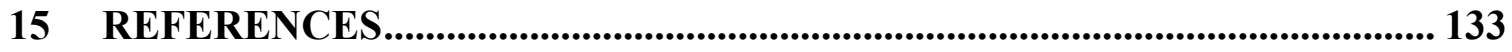




\section{List of Figures}

FIgURE 1: MASSES OF THE SIX QUARK FLAVORS. THE CURRENT AND ADDITIONAL QCD MASSES ARE SHOWN BY BLUE- AND YELLOW-BOXES, RESPECTIVELY. THE FIGURE IS ADAPTED FROM REF. [12].

FIGURE 2: CONTRIBUTIONS TO CHARM PRODUCTION AT RHIC ENERGIES INCLUDE THE INITIAL GLUON FUSION AND $Q \bar{Q}$ ANNIHILATION PHASE (SOLID LINE), A PRE-THERMAL PHASE CHARACTERIZED BY SCATTERING BETWEEN FREE-STREAMING PARTONS, AND A THERMAL PHASE THAT ASSUMES PARTON EQUILIBRATION. THE DISTRIBUTIONS WERE CALCULATED WITH AN ENERGY DENSITY OF $3.2 \mathrm{GEV} / \mathrm{FM}^{3}$ AT THE MOMENT OF THERMAL EQUILIBRATION. AS A REFERENCE, THE FAINT DOTTED LINE IS THE THERMAL PRODUCTION OF CHARM DUE TO AN INITIAL FULLY EQUILIBRATED QGP. THE FIGURE IS FROM REF. [15]. 22

FIGURE 3: TOTAL Cㅡㅡ PRODUCTION CROSS-SECTIONS PER NUCLEON-NUCLEON COLLISION VS. COLLISION ENERGY. THE DASHED LINE DEPICTS A PYTHIA CALCULATION. THE DOT-DASHED LINE DEPICTS A NLO PQCD CALCULATION WITH MRST HO, $\mathrm{M}_{\mathrm{C}}=1.2$ $\mathrm{GEV} / \mathrm{C}^{2}, \mu_{\mathrm{F}}=2 \mathrm{M}_{\mathrm{C}}, \mu_{\mathrm{R}}=2 \mathrm{M}_{\mathrm{C}}$. THE FIGURE IS ADAPTED FROM REF. $[17,19] \ldots \ldots \ldots \ldots . . . .22$

Figure 4: SOLID- AND DASHED-CURVES REPRESENT THE CHARM- (RED) AND BEAUTYHADRON (BLUE) SPECTRA FROM BLAST-WAVE AND PYTHIA MODEL CALCULATIONS, RESPECTIVELY. THE CORRESPONDING HEAVY FLAVOR DECAYED ELECTRON SPECTRA ARE SHOWN AS BLACK CURVES. THE DATA ARE THE MEASURED SINGLE ELECTRON DISTRIBUTIONS MEASURED IN 10\% CENTRAL AU + AU COLLISIONS AT $130 \mathrm{GEV} \mathrm{BY}$ THE PHENIX COLLABORATION. THE FIGURE IS ADAPTED FROM REF. [20]. 23

FIGURE 5: NUCLEAR MODIFICATION FACTOR RAA OF D-MESONS ASSUMING A HYDRODYNAMICALLY INSPIRED PARAMETERIZATION ASSUMING A COLLECTIVE FLOW VELOCITY OF $<\beta_{\mathrm{R}}>=0.4 C$ AND 0.6C FOR D-MESONS. THE CORRESPONDING ELECTRON DECAY-SPECTRA ARE SHOWN BY THE BLUE DASHED (1) AND (2) LINES. 24

FiguRE 6: ELLIPTIC FLOW OF STRANGE HADRONS AT RHIC AS MEASURED BY THE STAR DETECTOR. THE TOP PANEL DEMONSTRATES TYPICAL HYDRO-DYNAMICAL MASS ORDERING UP TO A MOMENTUM $\mathrm{P}_{\mathrm{T}}<2 \mathrm{GEV} / \mathrm{C}$ AND SATURATION AT LARGER MOMENTUM. THE BOTTOM PANEL SHOWS THE SCALING OF ELLIPTIC FLOW WITH THE NUMBER N OF CONSTITUENT QUARKS IN THE SATURATION REGION (BARYONS, N=3; MESONS, $\mathrm{N}=2$ ). 27

Figure 7: NON-PHOTONIC ELECTRON ELLIPTIC FLOW AT RHIC. OPEN AND ClOSED SYMBOLS ARE DATA OF NON-PHOTONIC ELECTRON V2 FROM PHENIX ${ }^{35}$ STATISTICAL ERRORS ARE SHOWN AS SOLID LINES AND THE OPEN BOXES INDICATE THE SIZE OF THE SYSTEMATIC ERRORS. THE SOLID-LINE REPRESENTS THE RESULTS FROM GRECO ET AL. WHERE, IN THIS COALESCENCE CALCULATION, SIMILAR V 2 DISTRIBUTIONS FOR C- AND U-QUARKS ARE ASSUMED. 28

FIGURE 8: THE RATIO OF SUPPRESSION FACTORS IN HOT MATTER FOR CHARM (H) AND LIGHT (L) QUARKS. THE SOLID LINE REPRESENTS RESULTS FROM CALCULATIONS WITH UNRESTRICTED GLUON RADIATION, WHILE THE DASHED LINE IS BASED ON CAlCulations With a CUT ON GLUON ENERGIES $\omega>0.5$ GeV. The Size of ThE 
STATIC MEDIUM TRAVERSED BY THE FAST QUARK IS ASSUMED TO BE 5 FM. THE FIGURE IS FROM REF. [49].

FIGURE 9: NON-PHOTONIC ELECTRON R AA IN CENTRAL AU + AU COLLISIONS FROM STAR (OPEN-SYMBOLS, TOP 5\%) AND PHENIX (FILLED-SYMBOLS, TOP 10\%) FROM REFS. $[56,57,58]$. THEORETICAL PREDICTIONS FOR CHARMED-HADRON AND CHARM AND BEAUTY-HADRON DECAYED ELECTRONS ARE SHOWN AS BLUE- AND YELLOW-BANDS, RESPECTIVELY. IN THESE CALCULATIONS, THE INITIAL GLUON DENSITY WAS ASSUMED TO BE UNREALISTICALLY LARGE: 3500, SEE REF [61] . 32

Figure 10: D-MESON CORRELATION FUNCTIONS FOR 200 GEV P + P COLLISIONS. DEFAULT PARAMETERS IN THE PYTHIA MODEL WERE USED IN THESE CALCULATIONS. A CLEAR BACK-TO-BACK CORRELATION IN THE ANGULAR DISTRIBUTION OF CHARMED MESONS IS OBSERVED (SHOWN BY THE OPEN CIRCLES). THE SOLID-LINE AND THE DIAMONDS REPRESENT THE RESULTS WITH ANGULAR SMEARING FOR $\sigma_{\phi}=\pi / 4$ AND $\pi / 2$, RESPECTIVELY. 33

FiguRE 11: LEFT TOP: TPC DE/DX VS. P IN D + AU COLLISIONS. LEFT BOTTOM: TPC DE/DX VS. P AFTER TOFR PID SELECTION OF $|1-\beta|<0.03$. ClEAN ELECTRON IDENTIFICATION IS ACHIEVED. RIGHT: DE/DX FROM THE TPC AFTER TOFR PID SELECTION FOR $1.0<\mathrm{P}<1.5 \mathrm{GEV} / \mathrm{C}$. 36

FIGURE 12: ELECTRON PT SPECTRA FROM $\gamma$ CONVERSIONS RECONSTRUCTED BY REQUIRING TPC TRACKING ALONE, A TACK IN THE TPC AND 2 HFT HITS. THE REJECTION FACTOR IS ABOUT 20. 37

Figure 13: Di-ElECtRon InVARIANT MASS SPECTRUM. THE SOLID BLACK CURVE IS A PREDICTION FROM R. RALF REF. [74] USING THE FULL STAR ACCEPTANCE. THE RED CURVE AT THE TOP IS THE TOTAL DI-ELECTRON INVARIANT MASS SPECTRUM SEEN WITH THE 2004 CONFIGURATION OF STAR BUT ASSUMING ELECTRON PID FROM FULL TOF COVERAGE. THE GRAY CURVE IS THE CHARM E+E- PAIR INVARIANT MASS DISTRIBUTION AFTER A CUT ON THE HFT DCA $<80$ UM. THE DOT DASHED LINE IS THE DALITZ DECAYS FROM $\pi 0$ AND $\eta$ AFTER REJECTION FROM TPC. 38

Figure 14: THE STAR DETECTOR AT RHIC. THE PRIMARY DETECTOR ELEMENTS AT MIDRAPIDITY ARE SHOWN IN THIS CUT-AWAY VIEW OF THE DETECTOR. FOR SCALE, THE TPC IS 4.2 METERS LONG AND 4 METERS IN DIAMETER. HEAVY ION BEAMS ENTER FROM THE LEFT AND RIGHT WHILE COLLISIONS TAKE PLACE IN THE CENTER OF THE DETECTOR. 40

FiguRE 15: LEFT - THE HFT IS SHOWN EMBEDDED INSIDE THE SSD. Right - SCHEMATIC VIEW OF THE HFT DETECTOR LADDER ARRANGEMENT WITH 6 LADDERS FORMING THE INNER LAYER AND 18 LADDERS FORMING THE OUTER LAYER. THE WIDTH OF THE LADDERS (THE DIMENSION SEEN IN THIS VIEW) IS 19 MM. THE SHINGLED LAYERS PROVIDE CROSS CALIBRATION OF THE LADDERS AND PROVIDE AN OPEN GEOMETRY FOR EASE OF INSTALLATION.

Figure 16: SimUlation BLOCK FLOW DIAGRAM. ONLY SMALL MODIFICATIONS WERE NEEDED TO OPTIMIZE THE STAR RECONSTRUCTION SOFTWARE FOR USE WITH THE HFT. 46 
FIGURE 17: DCA OF TRACKS TO THE PRIMARY VERTEX. THE GEOMETRIC SIGNED DISTANCE OF CLOSEST APPROACH BETWEEN ALL TRACKS $\left(\mathrm{P}_{\mathrm{T}}>200 \mathrm{MEV} / \mathrm{C}\right)$ AND THE VERTEX IS SHOWN FOR TWO CASES: A) DCA TO THE VERTEX DEFINED BY TPC+SSD TRACKS. THE NON-ZERO MEAN OF THIS DISTRIBUTION IS USED TO CORRECT THE VERTEX COORDINATE. B) DCA DISTRIBUTION AFTER ITERATIVE CORRECTION USING TPC+SSD+HFT TRACKS, WITH A SIGNIFICANT IMPROVEMENT IN THE MEAN AND SHAPE OF THE DISTRIBUTION. 47

FIGURE 18: MEAN AND SIGMA OF THE GEOMETRIC - SIGNED DCA DISTRIBUTION AS A FUNCTION OF THE NUMBER OF ITERATIONS USED TO CORRECT THE VERTEX. WITH EACH ITERATION, THE PREVIOUSLY DEFINED VERTEX IS CORRECTED WITH THE MEAN OF THE DISTRIBUTION OF THE TRACK DCA'S. THE FIRST ITERATION USES THE TPC+SSD DEFINED VERTEX AS A VERTEX SEED. 47

Figure 19: Vertex Resolution o(MC Vertex Position - RECONSTRUCTEd VerteX POSITION) AS A FUNCTION OF COLLISION CENTRALITY. THE HFT REFIT VERTEX SHOWS AN ORDER OF MAGNITUDE IMPROVEMENT OVER THE PREVIOUSLY RECONSTRUCTED PRIMARY VERTEX. A) VERTEX RESOLUTION IN X VS. $\mathrm{N}_{\mathrm{CH}}$. B) VERTEX RESOLUTION ALONG THE BEAM DIRECTION. PROBLEMS IN THE VERTEX FIT IN THE Z DIRECTION DRIVE THE DISTRIBUTION WIDER THAN IN THE TRANSVERSE DIRECTION. THE HIGHLIGHTED AREA DEMARCATES THE CENTRALITY REGION WITH MINIMUM (OR BETTER) ACCEPTABLE TPC+SSD VERTEX RESOLUTION. THIS CORRESPONDS TO 0\%$60 \%$ MOST CENTRAL EVENTS. 48

FIGURE 20: THE EFFICIENCY FOR FINDING TRACKS IN CENTRAL AU + AU COLLISIONS IN THE STAR TPC, SSD, AND THE HFT. ACCEPTED TRACKS HAVE 15 TPC HITS (OR MORE), 1 SSD HIT, AND 2 HFT HITS THAT MATCH TO A SINGLE MONTE CARLO TRACK. THE QUOTED EFFICIENCY IS FOR PIONS IN THE DETECTORS ACCEPTANCE; $|\eta|<0.5 \ldots . .49$

Figure 21: RECONSTRUCTED TRACKS WITH FALSE HFT HITS. LEFT - THE NUMBER OF GHOST TRACKS AND RECONSTRUCTED TRACKS PASSING THROUGH THE PIXELS OF THE HFT AS A FUNCTION OF P P. RIGHT - THE RATIO OF GHOST TRACKS TO GOOD TRACKS. THE INCREASED GHOSTING RATE AT LOWER TRANSVERSE MOMENTUM IS A DIRECT CONSEQUENCE OF MULTIPLE COULOMB SCATTERING, AND IS THE MOTIVATING FACTOR TO MAKE THE DETECTOR AS THIN AS POSSIBLE. 50

Figure 22: EFFiCIENCY (A) AND GHOST RATE (B) OF THE HFT, USING AN ANALYTIC CALCUlATIONS. THE LUMINOSITY DEPENDENCE IS GIVEN IN (C) AND (D) FOR 1-4 TIMES THE CURRENT PEAK LUMINOSITY. FINITE AND INFINITE SEARCH WINDOWS ARE REPRESENTED BY SOLID- AND DASHED- LINE, RESPECTIVELY. THE EFFICIENCY AND GHOST RATE FOR EACH LAYER OF THE HFT ARE CALCULATED SEPARATELY AND COMBINED INTO THAT FOR FULL HFT. THE VERTEX, SSD, HFT, AND TPC SEARCH RADII ARE ASSUMED TO BE $300 \mathrm{UM}, 60 \mathrm{UM}, 90 \mathrm{UM}$, AND $1.5 \mathrm{MM}$, RESPECTIVELY. AT NOMINAL RHIC LUMINOSITY $1 \times 10^{27} / \mathrm{CM}^{2} \mathrm{~S}$, THE DENSITY OF BACKGROUND HITS (HITS/CM ${ }^{2}$ ) ARE SUMMARIZED IN TABLE 10. IT WILL BE NECESSARY TO UPGRADE THE SENSORS FOR THE FULL RHICII LUMINOSITIES. 52 
Figure 23: THE DISTANCE OF CLOSEST APPROACH (DCA) DISTRIBUTIONS FOR $\mathrm{D}^{0}$ (TRIANGLES) AND $\mathrm{D}^{+}$(CIRCLES). THE RESUlTS OF THE DCA DISTRIBUTION FOR PRIMARY TRACKS ARE SHOWN IN SQUARES.

Figure 24: THE DECAY TOPOLOGY FOR A $\mathrm{D}^{0}$ DECAYING TO A KAON AND A PION. ISOLATION CUTS TO IDENTIFY THE $\mathrm{D}^{0}$ FROM THE BACKGROUND TRACKS ARE DESCRIBED IN THE TEXT.

FIGURE 25: DISTRIBUTION OF QUANTITIES USED TO DISTINGUISH SIGNAL FROM BACKGROUND. PANELS A1, A2, B1, AND B2 COMPARE THE DIFFERENCE BETWEEN BACKGROUND EVENTS AND D0 MESONS FOR DIFFERENT VARIABLES. THE YELLOW SHADED AREA SHOWS THE REGIONS WHERE WE PLACES CUTS TO ENRICH THE D0 TO BACKGROUND RATIO. THE LOWER PANELS, C1, C2, AND C2, SHOW THE PT DISTRIBUTION FOR SIGNAL AND BACKGROUND EVENT TRACKS WITH A $100 \mu \mathrm{M}$ ERROR

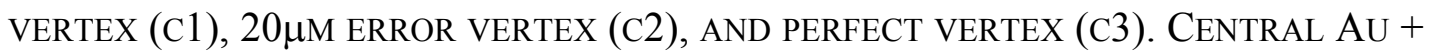
AU COLLISIONS ARE ASSUMED FOR THE BACKGROUND PRIMARY TRACKS.

FIGURE 26: $\mathrm{D}^{0}$ INVARIANT MASS WITH DIFFERENT CUT SETS. THE POWER OF THE CUTS TO ENHANCE THE SIGNAL RELATIVE TO THE BACKGROUND IS CLEAR. 57

Figure 27: THE ABSOLUTE YIELD OF $\mathrm{D}^{0}$ INTO THE TPC + SSD + HFT DIVIDED BY THE INPUT $\mathrm{D}^{0}$ YIELD. FOR COMPARISON, A SIMULATION WITH THICK SI DETECTORS THAT ARE SIMILAR TO THE WAFERS USED IN THE ALICE VERTEX DETECTOR IS ALSO SHOWN (1000 $\mu \mathrm{M}$ EFFECTIVE SILICON THICKNESS).

Figure 28: THE $\mathrm{D}^{+}$DECAYED TRACKS DECAY-LENGTH, DCA, AND COS( $\left.\theta\right)$ DISTRIBUTIONS (TRIANGLES). THE SAME DISTRIBUTIONS FOR PRIMARY TRACKS ARE ALSO SHOWN (SQUARES). 59

FIGURE 29: INVARIANT MASS DISTRIBUTIONS FOR $\mathrm{D}^{+}$FOR SEVERAL $\mathrm{P}_{\mathrm{T}}$ WINDOWS. THE LINES ARE A POLYNOMIAL (UP TO $2^{\mathrm{ND}}$ ORDER) + GAUSSIAN FIT. 59

FIGURE 30: D-MESON EFFICIENCIES AS A FUNCTION OF TRANSVERSE MOMENTUM, TOGETHER WITH FITS. TO THE DISTRIBUTIONS. THE D ${ }^{0}$ EFFICIENCY APPROACHES $10 \%$, WHILE THE $\mathrm{D}^{+}$EFFICIENCY APPROACHES . $3 \%$.

FiguRE 31: D-MESON RATES ESTIMATES: (A) DN/DP T DISTRIBUTIONS FOR D-MESONS. THE INTEGRATED YIELD DN/DY $=0.03$ AS MEASURED IN P + P COLLISIONS AT $200 \mathrm{GEV}$ [REF. 17]. THE NUMBER OF BINARY COLLISIONS $\left(\mathrm{N}_{\text {BIN }}\right)=950$, CORRESPONDS TO THE TOP 10\% MOST CENTRAL AU + AU COLLISIONS AT RHIC AND IS USED TO SCALE THE $\mathrm{Au}+$ AU COLLISIONS; (B) 3- $\sigma$ SIGNIFICANCE D ${ }^{0}$ EFFICIENCY WITH TPC+SSD+HFT. THE DASHED-LINE IS THE FIT TO THE SIMULATION RESULTS; (C) D $\mathrm{D}^{0}$ MESON RATES FROM P + P AND TOP 10\% CENTRAL AU + AU COLLISIONS AT $200 \mathrm{GEV}$. 61

FigURE 32: D-MESON V 2 RATE ESTIMATES: (A) DN/DP T DISTRIBUTIONS FOR D-MESON. THE INTEGRATED YIELD DN/DY $=0.03$ AS MEASURED IN P + P COLLISIONS AT 200 GEV [REF. 17]. THE NUMBER OF BINARY COLLISIONS $\mathrm{N}_{\text {BIN }}=290$, CORRESPONDING TO THE MINIMUM BIAS AU + AU COLLISIONS, IS USED TO SCALE THE AU + AU COLLISIONS. A POWER-LAW SHAPE FROM $\mathrm{P}+\mathrm{P}$ COLLISIONS IS ASSUMED; (B) ASSUMED $\mathrm{V}_{2}$ DISTRIBUTIONS FOR D-MESONS. THE SOLID-LINE IS THE RESULTS OF FIT TO THE MEASURED KAON V 2 [REF. 23]. BOTH CIRCLE- AND DIAMOND-SYMBOLS ARE FROM 
[REF. 46] FOR THE CASE WITH AND WITHOUT CHARM QUARK FLOW, RESPECTIVELY. ERROR BARS SHOWN ARE FROM 15\% SYSTEMATIC ERRORS; (C) $3-\sigma$ SIGNIFICANCE D ${ }^{0}$ EFFICIENCY WITH TPC+SSD+HFT. THE DASHED-LINE IS THE FIT TO THE SIMULATED RESULT; (D) $\mathrm{D}^{0}$ MESON V2 RATES FROM MINIMUM BIAS AU + AU COLLISIONS AT 200 GEV. THE SMALL AND LARGE ERROR BARS ARE FOR 15\% AND 30\% SYSTEMATIC ERRORS, RESPECTIVELY. FOR THE V 2 ANALYSIS, 12 BINS IN $\varphi$ ARE USED. 63

FIGURE 33: $\Lambda_{\mathrm{C}}$ DECAY LENGTH, DCA, AND INVARIANT MASS DISTRIBUTIONS. 64

FIGURE 34: EPITAXIAL SILICON USED AS A SENSOR. IN THIS DESIGN, A PRIMARY IONIZING PARTICLE CREATES FREE CHARGES IN THE EPITAXIALY GROWN SI LAYER THAT IS A FEW TENS OF MICRONS THICK AND A FEW ELECTRONS IN THE BULK LAYERS. THE LIBERATED CHARGES ARE THEN FREE TO DIFFUSE TOWARDS A POTENTIAL WELL STRUCTURE AT THE TOP OF THE SENSOR WHERE THEY ARE EXTRACTED AND READOUT INTO A DAQ SYSTEM. 70

FIGURE 35: WAFER OF RETICLE SIZE SENSORS (LEFT) AND ZOOMED-IN VIEW OF INDIVIDUAL CHIPS (RIGHT). 71

Figure 36: An APS SENSORS DEVElOPED By THE LBNL/UIC GROUP. THE PICTURE SHOWS 16 SEPARATE TEST STRUCTURES. EACH STRUCTURE HAS A $36 \times 36$ ARRAY OF $20 \mu \mathrm{M}$ PIXELS. 73

FIGURE 37: THIS GRAPH SHOWS THE RESULTS OF ONE EVENT TAKEN WITH $1.5 \mathrm{GEV}$ ELECTRONS. EACH BIN REPRESENTS ONE PIXEL AND THE HEIGHT IS PROPORTIONAL TO THE MEASURED CHARGE. SEVERAL ELECTRON HITSELECTRONS CAN BE IDENTIFIED IN THE PLOT. 73

FigurE 38: LADDER LAYOUT - SKETCH OF THE READOUT-TOPOLOGY ON A DETECTOR LADDER. THIS FIGURE SHOWS THE TEN APS AND THE CORRESPONDING CURRENT TO VOLTAGE CONVERSION AND DRIVER ELECTRONICS. THE DRIVERS WILL BE LOCATED OUT OF THE LOW MASS REGION OF THE DETECTOR AND MAY REQUIRE ADDITIONAL COOLING. 82

Figure 39: PRototype DAQ LAYOUT: SCHEMATIC OF DAQ SYSTEM FOR A SINGLE MIMOSTAR4 LADDER. ANALOG DATA IS CARRIED AS DIFFERENTIAL CURRENT ON THE LOW MASS CABLE AT $50 \mathrm{MHZ.} \mathrm{THE} \mathrm{SIGNALS} \mathrm{ARE} \mathrm{DRIVEN} \mathrm{IN} \mathrm{PARALLEL} \mathrm{OVER}$ SHORT $(\sim 1 \mathrm{M})$ TWISTED PAIR CABLES TO THE MOTHERBOARD. ANALOG TO DIGITAL CONVERSION, CDS AND DATA REDUCTION ARE PERFORMED IN THE MOTHERBOARD / DAUGHTER CARDS. THE REDUCED HIT DATA IS TRANSFERRED DIGITALLY TO THE SIU AND CARRIED TO LINUX BASED READOUT PCS VIA AN OPTICAL FIBER. CONTROL, SYNCHRONIZATION, AND EVENT ID TAGGING ARE ACCOMPLISHED IN THE SYNCH/TRIGGER FPGA ON THE MOTHERBOARD. 83

FIGURE 40: A SIMPLE CLUSTER FINDING ALGORITHM FOR THE HFT DETECTOR. ADC DATA FROM TWO MIMOSTAR DETECTOR COLUMNS + 3 PIXELS ARE SENT TO A HIGH/LOW THRESHOLD DISCRIMINATOR. THE RESULTING 2 BITS ARE FED SEQUENTIALLY IN AN 2BIT WIDE SHIFT REGISTER. THE CENTER PIXEL OF A $3 \times 3$ PIXEL WINDOW IS COMPARED TO A HIGH THRESHOLD WITH EACH CLOCK TICK. IF THE THRESHOLD IS EXCEEDED, THE ADDITIONAL CLUSTER IDENTIFICATION CRITERIA ARE CHECKED FOR THE 3 X 3 PIXEL 
WINDOW. IF THE RESULTS MEET THE CRITERA FOR A CLUSTER, THE CENTER PIXEL ADDRESS IS STORED INTO A READOUT FIFO. THIS METHOD IS EXTENDABLE TO ALLOW FOR MULTIPLE SIMULTANEOUS THRESHOLDS AND GEOMETRIC PATTERN TRIGGERS... 84

FIGURE 41: EFFICIENCY VERSUS ACCIDENTALS FOR A CLUSTER FINDING ALGORITHMS RUN ON CLUSTER DATA FROM A MIMOSA5 DETECTOR. NOTE THAT SOME PARAMETER COMBINATIONS OF THIS ALGORITHM ARE ALREADY OVER 98\% EFFICIENT WITH A ACCIDENTALS RATE OF 1-2 HITS / $\mathrm{CM}^{2}$. 85

Figure 42: MULTIPLE EVENT FIFOS ARE FED IN PARALLEL FROM THE CLUSTER FINDER. A SEPARATE EVENT FIFO IS ENABLED FOR ONE FRAME UPON THE RECEIPT OF A TRIGGER FROM THE TCD. THE RESULTING SEPARATE COMPLETE FRAMES ARE THEN PASSED TO STAR DAQ AS THEY ARE COMPLETED IN THE EVENT FIFOS. 86

Figure 43: DATA RATES AT the VARIOUs STAgES OF THE PROTOTYPE MIMOSTAR4 READOUT CHAIN.

FIGURE 44: FUNCTIONAL BLOCK DIAGRAM FOR ULTIMATE SENSOR BASED READOUT SYSTEM. 88

FIGURE 45: DATA RATES IN ULTIMATE HFT READOUT. 89

FiguRE 46: A PROTOTYPE LADDER SHOWING LOW MASS PCB, MIMOSA5 DETECTORS AND DRIVER ELECTRONICS BONDED TO A MECHANICAL CARBON FIBER AND RETICULATED VITREOUS CARBON FOAM BASED CARRIER. 90

FIGURE 47: FUNCTIONAL COMPONENT DIAGRAM OF THE PROTOTYPE READOUT SYSTEM CONSTRUCTED FOR THE READOUT ON A MIMOSA5 BASED LADDER. 91

Figure 48: EARLY PROTOTYPE MOTHERBOARD AND DAUGHTER CARD USED FOR READING OUT MIMOSA5 DETECTORS. 92

FIGURE 49: THE HFT IS SHOWN INTEGRATED WITH THE STAR INNER DETECTORS CONE ASSEMBLY 94

Figure 50: Close UP VIEW OF THE HFT LADDERS. 95

FIGURE 51: DETECTOR SUPPORT STRUCTURE WITH KINEMATIC MOUNTS TO INSURE REPEATABLE DETECTOR POSITIONING. 95

FIGURE 52: KINEMATIC MOUNT STRUCTURE. 96

FIGURE 53: THE HFT IS SHOWN INTEGRATED WITH THE STAR INNER DETECTORS CONE ASSEMBLY.

Figure 54: LADDER ASSEMBLY. THE CURRENT LADDER IS COMPOSED OF A ROW OF 10 THINNED APS DETECTORS ON TOP OF A MATCHING ROW OF THINNED READOUT CHIPS AND A THREE-LAYER ALUMINUM KAPTON CABLE. THE SILICON CABLE STRUCTURE IS BONDED TO A CARBON COMPOSITE "V", CLOSING THE BEAM TO MAKE A RIGID STRUCTURE.

FIGURE 55: A CROSS-SECTION OF THE PROTOTYPE DETECTOR LADDER SHOWING ITS STRUCTURE AND MATERIALS COMPOSITION. 100 
FIGURE 56: A CROSS-SECTION OF THE PROTOTYPE DETECTOR LADDER SHOWING ITS STRUCTURE AND MATERIALS COMPOSITION.

FIGURE 57: MEASURED BEND OF A SILICON/CARBON COMPOSITE LADDER TEST STRUCTURE AND THE CALCULATED BEND SHAPE. THE LADDER WAS RIGIDLY SUPPORTED AT ONE END WITH A 10 GRAM WEIGHT PLACED ON THE OTHER. 101

FIGURE 58: TV HOLOGRAPHY SYSTEM VIEWING TEST LADDER IN A SMALL TRANSPARENT WIND TUNNEL. INSET SHOWS DIFFRACTION PATTERN WITH COLOR MAP OF THE SURFACE DISPLACEMENT. . 102

FIGURE 59: A PROTOTYPE READOUT CABLE FOR THE HFT …........................................ 103

Figure 60: Mechanical Prototype With 4 MiMOSA-5 Detectors Glued to the KAPTON CABLE ASSEMBLY. 104

FIGURE 61: BEAM PIPE STRUCTURE WITH OUTER SUPPORT BARREL IN THE CENTER. ....... 105

FIGURE 62: VIEW OF THE BEAMPIPE MID-SECTION. 106

FIGURE 63: END VIEW SHOWING THE HFT LADDERS BETWEEN SPOKES TO THE INNER BEAM PIPE SUPPORT.

FiguRE 64: A SCHEMATIC VIEW OF THE INSTALLATION ACTIVITIES IN EACH YEAR. INSTALLATION IS TYPICALLY DONE IN THE SUMMER OF EACH YEAR. 110

FIGURE 65: SCHEDULE OVERVIEW AND ROLL-UP OF ACTIVITIES. 111

FIGURE 66: DETAIL FROM THE GANTT CHART THAT TRACKS THE FULL ENTERPRISE OF HFT ACTIVITIES. 


\section{List of Tables}

TABLE 1: CROSS SECTION FOR THE PRODUCTION OF CHARM AND BEAUTY

TABle 2: Charm QUARK FRAGMENTATION FUnCTIONS. ThE LEFT COLUMN IS FROM REF. [44,45]. The Right COLUMN IS FrOM ReF. [43]. THE $\mathrm{D}^{+}$AND $\mathrm{D}^{0}$ YIELDS InClude FEED-DOWN FROM $\mathrm{D}^{*+}$ AND $\mathrm{D}^{* 0}$ DECAYS.

TABLE 3: THE NUMBER OF VECTOR MESON PAIRS WHICH CAN BE RECORDED BY STAR IN ONE RHIC YEAR. WE ASSUME 200 M CENTRAL AU + AU EVENTS WILL BE RECORDED PER RHIC YEAR WHEN DAQ 1000 IS OPERATIONAL. 38

TABLE 4: SElECTED HFT PARAMETERS FOR THE DETECTOR WE PROPOSE TO BUILD. THE SIMULATED PARAMETERS ARE THE SAME EXCEPT THAT WE HAVE ASSUMED THAT THE DETECTOR IS $0.38 \%$ RADIATION LENGTHS THICK, PER LAYER, IN ORDER TO ALLOW FOR THE POSSIBILITY OF AN ONBOARD READOUT CHIP. 43

TABLE 5: OPEN CHARM HADRON PROPERTIES 53

TABle 6: THE CUTS FOR THE D0 AND D+ RECONSTRUCTION AND EFFICIENCY ANALYSIS. THE INPUT D0 AND D+ SPECTRA FOLLOWED AN EXPONENTIAL DISTRIBUTION, $<\mathrm{PT}>=1.3 \mathrm{GEV} / \mathrm{C}$. 56

TABLE 7: AN ESTIMATE OF THE NUMBER OF EVENTS REQUIRED TO ACHIEVE 10\% STATISTICAL ERRORS FOR THE SPECTRA MEASUREMENTS OF 200 GEV P + P COLLISIONS AND $0-10 \% / 0-80 \%$ AU + AU COLLISIONS. A POWER-LAW SHAPE PT DISTRIBUTION FROM THE P + P COLLISION HAS BEEN ASSUMED WITH THE MID-RAPIDITY DN/DY $=0.03$ [REF. 17]. THE NUMBER OF BINARY COLLISIONS IS FROM GLAUBER CALCULATIONS [REF. 23].

TABLE 8: AN ESTIMATE OF THE NUMBER EVENTS REQUIRED FOR 10\% STATISTICAL ERRORS FOR D ${ }^{0}$ V2 MEASUREMENTS OF $200 \mathrm{GEV} \mathrm{0-80 \%} \mathrm{AU} \mathrm{+} \mathrm{AU} \mathrm{COLLISIONS}\left(\mathrm{N}_{\text {BIN }}=290\right)$. A POWER-LAW SHAPE PT DISTRIBUTION FROM THE P + P COLLISION HAS BEEN ASSUMED WITH THE MID-RAPIDITY DN/DY $=0.03$ [REF. 17]. THE NUMBER OF BINARY COLLISIONS IS FROM THE GLAUBOR CALCULATIONS [REF. 23]. 62

TABLE 9: LUMINOSITY AND OTHER PARAMETERS THAT DETERMINE THE PARTICLE FLUX ON THE HFT. 65

TABLE 10: INTEGRATED HIT LOADING ON THE HFT AND ASSOCIATED PILEUP. 65

TABle 11: Hit loAding on the HFT From Au + Au CENTRAL COLLISIONS AND ASSOCIATED PILEUP.

TABle 12: TABLE - CHART OF APS CHIPS THAT THE IRES GROUP HAS PRODUCED IN THE PAST 5 YEARS [].

TABLE 13: COMPARISON BETWEEN MIMOSA-5 CHARACTERISTICS AND MIMOSTAR-4 SPECIFICATIONS.

TABle 14: Silicon REQUiREMENTS For MAXIMUM AVERAGE Au+Au RHIC LUMINOSITY OF $7.0 \times 10^{27} \mathrm{~Hz} / \mathrm{CM}^{2}$ OR $2.5 \mathrm{NB}^{-1} /$ WEEK 
Table 15: Prototype Stage Requirement Summary - Constraints for the

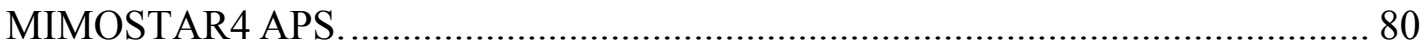

TABLE 16: DATA RATE CALCULATION PARAMETERS....................................................... 87

Table 17: Final Stage Specification Summary - CONSTRaints for the ULTRA

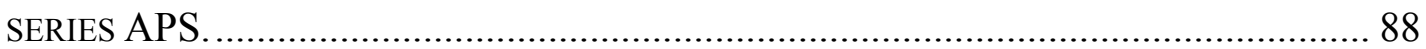

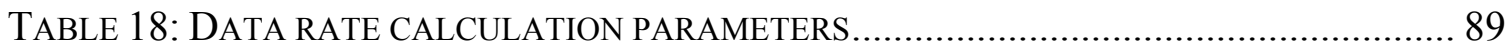

TABLE 19: MATERIALS IN THE BEAM PIPE AND THE FIRST DETECTOR LAYER WITH THEIR TOTAL THICKNESS AND RADIATION LENGTH. FOR DETAILS SEE REF. [] ..................... 99

TABle 20: Estimated COST OF THE HFT COMPONENTS. THE tOTAL COST FOR THE HARDWARE COMPONENTS IS $\$ 3.75 \mathrm{M}$. THIS DOES NOT INCLUDE ENGINEERING LABOR BUT IT DOES INCLUDE APPROXIMATELY \$1M IN MANUFACTURING LABOR TO BE SPENT IN THE MECHANICAL SHOPS AT BNL AND LBL. 114

Table 21: Proposed Funding Profile for the HFT that appears in the BNL Mid TERM PLAN ${ }^{136}$ 115 


\section{Executive Summary}

We propose to construct a Heavy Flavor Tracker (HFT) for the STAR experiment at RHIC. The HFT will bring new physics capabilities to STAR and it will significantly enhance the physics capabilities of the detector at central rapidities.

The HFT will use CMOS Active Pixel Sensors (APS) which are an innovative new technology which have never been used before in a collider experiment. Our APS sensors are only $50 \mu \mathrm{m}$ thick and this opens up a new realm of possibilities for physics measurements. In particular, a thin detector $(0.28 \%$ radiation length per layer $)$ in STAR makes it possible to do the direct topological reconstruction of open charm hadrons by the identification of the charged daughters of the hadronic decay. So, for example, we can directly identify the decays $\mathrm{D}^{0} \rightarrow \mathrm{K}^{-} \pi^{+}$and $\mathrm{D}_{\mathrm{s}}^{+} \rightarrow \mathrm{K}^{-} \pi^{+} \mathrm{K}^{+}$by identifying the kaons and pions with the rest of STAR and then using the pointing resolution of the HFT to find the daughters with a common decay-vertex but where this vertex is displaced by $100 \mu \mathrm{m}$ from the primary vertex.

\subsection{Scientific Motivation}

The primary motivation for the HFT is to extend STAR's capability to measure heavy flavor production by the measurement of displaced vertices and to do the direct topological identification of open charm hadrons. These are key measurements for the heavy ion and spin physics programs at RHIC. Heavy quark measurements will facilitate the heavy ion program as it moves from the discovery phase to the systematic study of the dense medium created in heavy ion collisions as well as the nucleon spin structure in polarized $p+p$ collisions. The primary physics topics to be addressed by the HFT include heavy flavor energy loss, flow and a test of partonic thermalization at RHIC.

A precise measurement of the spectra of D meson states will shed light on several open questions in heavy ion collisions. From the spectra and the production ratios of D states we will be able to extrapolate to the total yield for charm quark production. Furthermore, the open charm production rate is high enough at RHIC that the coalescence process becomes relevant for Charmonium production. Knowledge of the total production cross section for charm quarks is also essential as a baseline for $\mathrm{J} / \psi$ measurements. A meaningful answer to the question of whether the $\mathrm{J} / \psi$ mesons are suppressed or enhanced at RHIC requires knowledge of the charm production in heavy ion reactions.

The heavy quark can also be used to probe the properties of the medium created in heavy ion collisions. The production of gluons is kinematically suppressed for heavy flavors (due to the dead cone effect). As a consequence, heavy flavors should lose less energy in the dense medium. 
An important measurement to be made with the HFT is $\mathrm{R}_{\mathrm{AA}}$; the ratio of charmed meson production in $\mathrm{Au}-\mathrm{Au}$ collisions to the binary-scaled production rate in $\mathrm{p}-\mathrm{p}$ or $\mathrm{d}-\mathrm{Au}$ collisions. (See section 2.5.1). Current measurements using non-photonic electrons as a measure of the abundance of charm and beauty hadrons, indicate that the rate of energy loss for heavy quarks is unexpectedly high and inconsistent with our current understanding in pQCD models. Based on the non-photonic electron data presented at Quark Matter, the whole theory of heavy quark energy loss is uncertain and may be completely wrong; especially in regards to beauty.

Another important measurement to be made with the HFT is a measurement of the elliptic flow of D mesons down to very low $\mathrm{p}_{\mathrm{T}}$ values. (See section 2.4.1). It is generally accepted that elliptic flow is established in the partonic phase. If charm quarks, with a mass much larger than the temperature of the system, undergo elliptic flow then it has to arise from many collisions with the abundant light quarks. Thus, flow of charm quarks can be taken as a probe for frequent re-scatterings of light quarks and is an indication of thermalization that may be reached in the early stages of heavy ion collisions at RHIC. We believe that proof of thermalization constitutes the last step towards the establishment of the QGP at RHIC and this measurement requires a very thin detector to push the measurement down to the lowest momenta where transverse elliptic flow is manifest.

The pixel detector offers the possibility of measuring $\mathrm{e}^{+} \mathrm{e}^{-}$pairs down to the vector meson mass region by removing the gamma conversion background, while electrons and positrons are identified in the TPC and TOF. (See section 2.6). Vector mesons reconstructed in the dilepton channel may have decayed at any stage of the systems evolution, since leptons are very unlikely to re-interact. Thus, studying the low mass $\mathrm{e}^{+} \mathrm{e}^{-}$ spectrum will yield crucial insights into the nature of the system before it reaches the low-density hadronic freeze-out stage. Measuring the low-mass vector mesons through their leptonic decay channel may also yield information about the onset of chiral symmetry restoration.

\subsection{Detector Concept}

The HFT detector brings extremely high precision tracking capabilities to STAR with a resolution of $10 \mu \mathrm{m}$ at the first layer of the detector, over a large pseudo-rapidity range, and with complete azimuthal angular coverage. The HFT will enable STAR to perform high precision measurements of heavy-quark production over the broadest range of phase space, colliding system sizes and energies. It will exploit all of STAR's unique features including particle ID and tracking from the lowest $\mathrm{p}_{\mathrm{T}}$ to the highest. In these respects, the HFT is unique at RHIC.

The HFT will sit inside the STAR TPC and it will surround the interaction vertex. The HFT has two tracking layers composed of monolithic CMOS pixel detectors using 
$30 \mu \mathrm{m} \times 30 \mu \mathrm{m}$ square pixels. These critical innermost tracking layers lie at radii of 1.5 $\mathrm{cm}$ and $5.0 \mathrm{~cm}$, respectively, and these layers are active over $20 \mathrm{~cm} \mathrm{in} \mathrm{z}$ and have $\sim 100$ million pixels. The HFT will provide tracking information for decaying particles that are displaced by 100 microns from the primary event-vertex. The silicon chips for the detector will be thinned to $50 \mu \mathrm{m}$ and will be mounted on low mass carbon fiber structures to minimize pointing errors generated by multiple Coulomb scattering.

Such a thin detector requires a correspondingly thin beam pipe. Therefore, we propose to build a new beam-pipe for the STAR detector which is only $0.5 \mathrm{~mm}$ thick. The construction of such a thin beam-pipe is challenging and it requires a unique design to enable the beampipe to be handled during installation and bakeout.

\subsection{R\&D for an Advanced Detector}

The HFT is an advanced detector. It explores new technologies that have never been used before at RHIC and therefore we propose to evaluate the technology, carefully, with an extended R\&D phase in the project plan. For example, the new beampipe needs to be designed and tested in collaboration with the BNL Collider Accelerator Divison. We have already started an extensive program of R\&D using LDRD funds from LBL, and contributed labor from the IRES group in Strasbourg, France, to develop the basic Si technology and chipset. Progress on this front is excellent and it is described in section 4, however, more work on the chips is required. The readout electronics must be compatible with the large data flow from the Si detectors, and even the software for calibration and tracking will be pushed to new limits. Finally, the mechanical systems to hold the chips and ladders must meet very demanding mechanical tolerances and so this will require advanced engineering prototyping and design. We feel that these challenges are interesting, fun, and can be surmounted but we propose to reduce the risk to the project by exploring these topics with an extended program of $R \& D$ activities.

\subsection{Coordination with Other New STAR Detectors}

The HFT is to be built in coordination with a new, high rate, Intermediate STAR Tracker. The primary purpose of the IST is to extend the tracks found in the TPC and to point them accurately at the outer layer of the HFT. Pointing with an accuracy of $125 \mu \mathrm{m}$, or less, is required in order to independently find hit points in the HFT without resorting to a vertex constraint on the track. Using a vertex constraint works in a high multiplicity environment and so ensures a baseline physics program for the HFT. But, a vertex constraint doesn't work for pp collisions or the most peripheral Au-Au interactions. So the IST is needed in order to collect the low multiplicity data needed to complete our flow measurement program and it is required in order to gather the reference data in $\mathrm{d}-\mathrm{Au}$ or $\mathrm{p}-\mathrm{p}$ collisions for the denominator in our $\mathrm{R}_{\mathrm{AA}}$ measurements. 


\section{The Physics of the HFT}

\subsection{Introduction}

An important goal of high-energy nuclear physics is to understand Quantum Chromodynamics (QCD) at extreme temperatures and energy densities. Under these extraordinary conditions, we believe that the fundamental symmetries of QCD will reveal themselves: quarks and gluons will be the relevant degrees of freedom, color will be deconfined and chiral symmetry will be restored. Calculations within the framework of regularized lattice QCD predicts a fast crossover from ordinary nuclear matter into a deconfined and locally thermalized state of quarks and gluons called the Quark-Gluon Plasma $^{1}$ (QGP).

High-energy nuclear collisions can be characterized by three distinct phases: the initial phase where hard interactions between the partons of the incoming nuclei dominate, an intermediate phase where re-interactions between the constituents in the matter result in collectivity, and a final stage where hadronization, and chemical and thermal freeze-out occur. The matter produced in high-energy nuclear collisions can be investigated by studying the dynamics of the collective expansion of the bulk of the produced particles and by studying the interaction of the medium with penetrating probes such as leading particles and jets. In particular, the measurement of large elliptic flow ${ }^{2}$, and the observation of strong modifications of high $p_{T}$ particle production as measured by the ratio $\mathrm{R}_{\mathrm{AA}}{ }^{3}$, and the disappearance of the away side jet ${ }^{4}$ have provided evidence for a high density and strongly interacting state of matter at RHIC ${ }^{5}$. The goal of our research program is to elucidate the nature of this matter and to determine if it is dominated by hadronic or partonic degrees of freedom.

Rare processes provide new ways to probe the medium generated in high-energy nuclear collisions. Bjorken ${ }^{6}$ proposed that hard scattered partons (quarks and gluons) drawn from the incoming nuclei will interact with the medium in a density-dependent way. Bjorken's initial energy loss mechanism (elastic scattering) did not provide effects large enough to be observed but medium-induced radiation (gluonic bremsstrahlung) can generate significant energy loss effects ${ }^{7,8}$. For example, hard parton scatterings can be experimentally reconstructed in elementary particle collisions $\left(e^{+}+e^{-}, \bar{p}+p\right)$ because the outgoing parton fragments into a collimated spray of energetic hadrons at large transverse angles with respect to the beam.

The cluster of hadrons, from the parton fragmentation, is known as a jet. Jets can also be identified in high energy nuclear collisions on a statistical basis; the modification of their properties may signal novel flavor dependent energy loss mechanisms in a dense medium by the parton that initiated the jet. Full jet reconstruction in heavy ion collisions is 
exceedingly difficult but leading hadrons (i.e. high $p_{\mathrm{T}}$ hadrons, which typically carry a large fraction of the jet energy) and their correlations with other hadrons may provide the essential signals of partonic interactions in the medium.

Collective flow measurements have played a prominent role in understanding the physics of nuclear collisions because the magnitude and pattern of the collective motion is closely related to the equation of state (EOS) of the produced matter.

If local equilibrium is achieved, we will be able to use hydrodynamic models to study the EOS and understand the degrees of freedom relevant for the basic constituents of the matter. Heavy quark $(c, b)$ production provides some of the most important observables. Due to their large masses, $c$ and $b$ quarks are produced dominantly by the interactions of the initial incoming partons whereas lighter quarks are produced throughout the later stages of the evolution of a heavy ion collision. Thus, the total yields of $c$ and $b$ quark production provide a direct connection to the initial state.

Due to their heavy mass and presumably small hadronic cross sections, charmed quarks are a sensitive probe for the frequency of interaction and therefore the degree of thermalization with constituents before hadronization. At high $p_{\mathrm{T}}$, heavy quarks may be less suppressed than light quarks due to the "dead cone" effect and so high $\mathrm{p}_{\mathrm{T}}$ probes are an additional way to study partonic energy loss. If charmed quarks participate sufficiently in re-scattering processes, they will develop flow (i.e. transverse radial and elliptic flow), which can be observed in charmed hadron momentum distributions. Finally, charmed quarks might achieve thermal equilibrium with the surrounding medium leading to statistical hadronization. In this case, the relative abundances of charmed hadrons are significantly modified.

The study of the structure of the nucleon is an important frontier in strong interaction physics. Despite considerable experimental and theoretical progress over the past several decades, many open questions remain. A striking example is the spin structure of the proton, which has been of key interest ever since the European Muon Collaboration published their data on the spin structure function $g_{1}\left(x, Q^{2}\right)^{9,10}$. The EMC data and subsequent data indicate that the quark helicity contribution to the proton spin is remarkably small. The natural question regarding the fraction of the nucleon spin carried by gluon helicities remains basically unanswered. Unlike previous nucleon spin measurements, STAR will observe processes where the polarized gluon distribution function $\Delta G\left(x, Q^{2}\right)$ contributes at leading order via a hadronic, not electromagnetic, probe. Heavy quark production is expected to provide direct access to gluons in the proton with a large analyzing power for longitudinal spins. 


\subsection{Initial Heavy Quark Production}

Quarks are elementary particles and, depending on the energy scale, there are two mechanisms that generate their masses with different degrees of importance: current quark masses are generated by the electroweak symmetry breaking mechanism (Higgs mass) and spontaneous chiral symmetry breaking leads to the constituent quark masses in QCD (QCD mass). The QCD interaction affects only the light quarks ( $u, d, s$ ) while the heavy quark masses $(c, b, t)$ are determined by the Higgs mechanism, see Figure 1. In high-energy nuclear collisions at RHIC, heavy quarks are produced through gluon fusion and $\mathrm{q} \overline{\mathrm{q}}$ annihilation ${ }^{11}$.

Heavy quark production is also sensitive to the parton distribution function. Unlike the light quarks, heavy quark masses are not modified by the surrounding QCD medium ${ }^{12}$ (or the excitations of the QCD medium) and the value of their masses is much higher than the initial excitation of the system. It is these differences between light and heavy quarks in a medium that make heavy quarks an ideal probe to study the properties of the hotdense medium created in high-energy nuclear collisions.

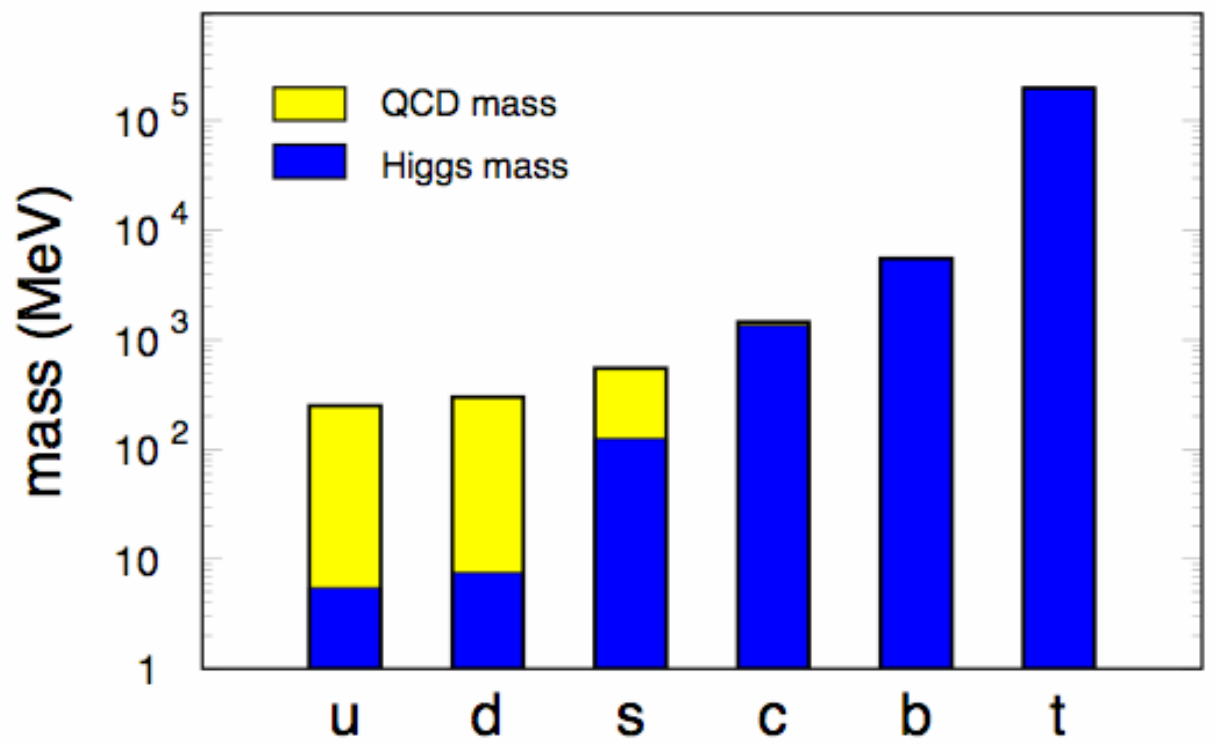

Figure 1: Masses of the six quark flavors. The current and additional QCD masses are shown by blue- and yellow-boxes, respectively. The figure is adapted from Ref. [12].

Understanding the yield of charmed hadrons in hadron-hadron collisions requires a knowledge of the projectile and target parton distribution functions, the cross section for parton-parton interactions which generate charm quarks and the fragmentation functions for $c(b)$ quarks into charmed (bottom) hadrons. The parton distributions within the proton can be extracted from electron-proton collisions while the cross-sections for gluon fusion and $q \bar{q}$ annihilation are calculated in a perturbative QCD framework up to next-to leading-order (NLO) ${ }^{13}$. However, the parton and gluon distribution functions within the 
nucleus, relevant to the charm and bottom quark production at RHIC energies, are poorly understood $^{14}$ and thus leave room for precise measurements of charm cross sections in $\mathrm{p}+\mathrm{p}, \mathrm{d}+\mathrm{Au}$ and $\mathrm{Au}+\mathrm{Au}$ collisions. Perturbative QCD predictions for the cross section $\sigma(c \bar{c})$ and $\sigma(b \bar{b})$ in $\mathrm{p}+\mathrm{p}$ collisions at ${\sqrt{\mathrm{S}_{\mathrm{NN}}}}=200 \mathrm{GeV}$ may be found in the literature ${ }^{13}$ and they are in reasonably good agreement with the cross-sections measured at RHIC given that some of the theoretical calculations are uncertain by as much as $50 \%$.

\begin{tabular}{|l|l|}
\hline Experiment: $\sigma_{\mathrm{NN}}(c \bar{c})=900-1400 \mu \mathrm{b}$ \\
Theory : $\quad \sigma_{\mathrm{NN}}(c \bar{c})=289-445 \mu \mathrm{b}$ \\
$20-30 c \bar{c}$ pairs per central $\mathrm{Au}+\mathrm{Au}$ collision at $\sqrt{\mathrm{S}}_{\mathrm{NN}}=200 \mathrm{GeV}$ \\
\hline Theory : $\quad \sigma_{\mathrm{NN}}(b \bar{b})=1.64-2.16 \mu \mathrm{b}$ \\
$0.04-0.06 b \bar{b}$ pairs per central Au + Au collision at $\sqrt{\mathrm{S}}_{\mathrm{NN}}=200 \mathrm{GeV}$ \\
\hline
\end{tabular}

Table 1: Cross section for the production of charm and beauty.

The uncertainty in the theoretical cross-sections arises from a reasonable variation of quark masses $\left(m_{c}=1.2-1.8 \mathrm{GeV}, m_{b}=4.5-5.0 \mathrm{GeV}\right)$, factorization and renormalization scales $\left(\mu_{\mathrm{R}}\right.$ and $\mu_{\mathrm{F}}$ ), and parton distribution functions (MRST, CTEQ, GRV). The number of underlying binary nucleon-nucleon collisions normalizes the cross section values. (There are approximately 1000 binary nucleon-nucleon scatterings for each central $\mathrm{Au}+\mathrm{Au}$ collision.)

In heavy ion collisions, final state interactions may also enhance charm production relative to the binary scaling of initial parton collisions and may also lead to additional production of charm via "thermal" processes. An analogous mechanism dominates strangeness production. The heavy quark channels should be greatly suppressed by the thermal factor due to the heavy quark mass $\left(m_{c} \approx 1.2 \text { to } 1.8 \mathrm{GeV}, m_{b} \approx 4.5 \text { to } 5.0 \mathrm{GeV}\right)^{15}$, making heavy quark production rates primarily sensitive to the dynamics of the initial collisions. This is especially true for the $\mathrm{b}$ quark. Figure 2 shows the calculated contributions to the total charm production at $y=0$ for ${\sqrt{S_{\mathrm{NN}}}}=200 \mathrm{GeV}$, with an energy density of $3.2 \mathrm{GeV} / \mathrm{fm}^{3}$ at the moment of thermal equilibration.

STAR and PHENIX have made measurements of charm production in $\mathrm{Au}+\mathrm{Au}, \mathrm{d}+\mathrm{Au}$, and $\mathrm{p}+\mathrm{p}$ collisions at RHIC. The PHENIX data for $\mathrm{Au}+\mathrm{Au}$ collisions suggests that the cross-section for open charm production is consistent with the expectations of $\mathrm{pQCD}$. These results were derived from non-photonic single electron spectra ${ }^{16}$ as shown in Figure 3. The STAR results show that the cross-section for open charm production in $\mathrm{d}+\mathrm{Au}$ and $\mathrm{Au}+\mathrm{Au}$ is consistent with binary collision scaling but the total yield may be larger than NLO pQCD models. Note, since we only measure the electrons and $\mathrm{D}^{0} \mathrm{~s}$ to extract the total charm cross-section, several assumptions such as the ratios of the neutral over charged D-mesons have to be used. Both the $\mathrm{p}_{\mathrm{T}}$ integrated yield of $\mathrm{dN} / \mathrm{dy}$ and the 
value of $\left\langle\mathrm{p}_{\mathrm{T}}\right\rangle$ of the measured $\mathrm{D}$-meson spectrum are larger than the pQCD model predictions. The STAR results are obtained from a direct reconstruction of the open charm hadrons ${ }^{17}$ as well as through single electron spectra.

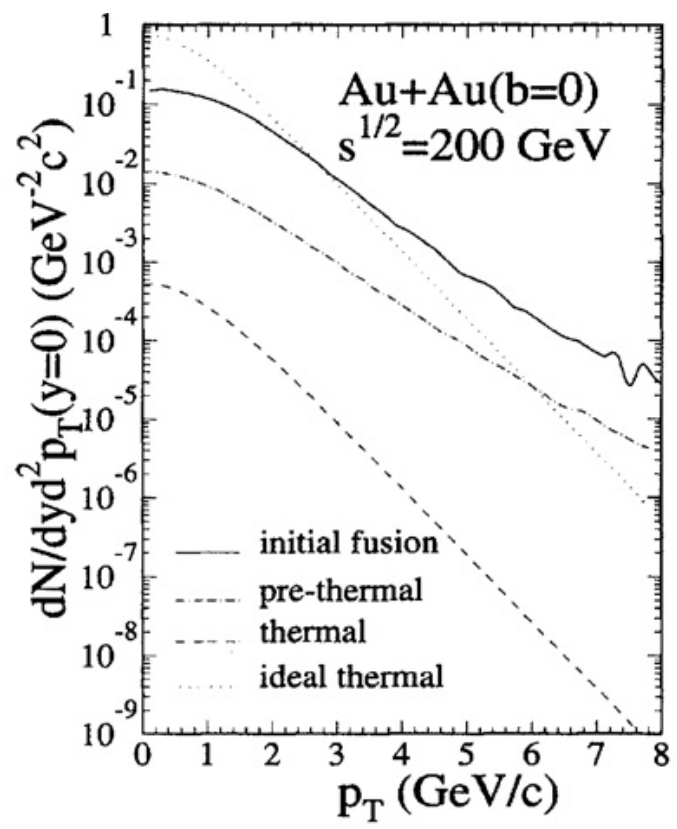

Figure 2: Contributions to charm production at RHIC energies include the initial gluon fusion and $q \bar{q}$ annihilation phase (solid line), a pre-thermal phase characterized by scattering between freestreaming partons, and a thermal phase that assumes parton equilibration. The distributions were calculated with an energy density of $3.2 \mathrm{GeV} / \mathrm{fm}^{3}$ at the moment of thermal equilibration. As a reference, the faint dotted line is the thermal production of charm due to an initial fully equilibrated QGP. The figure is from Ref. [15].

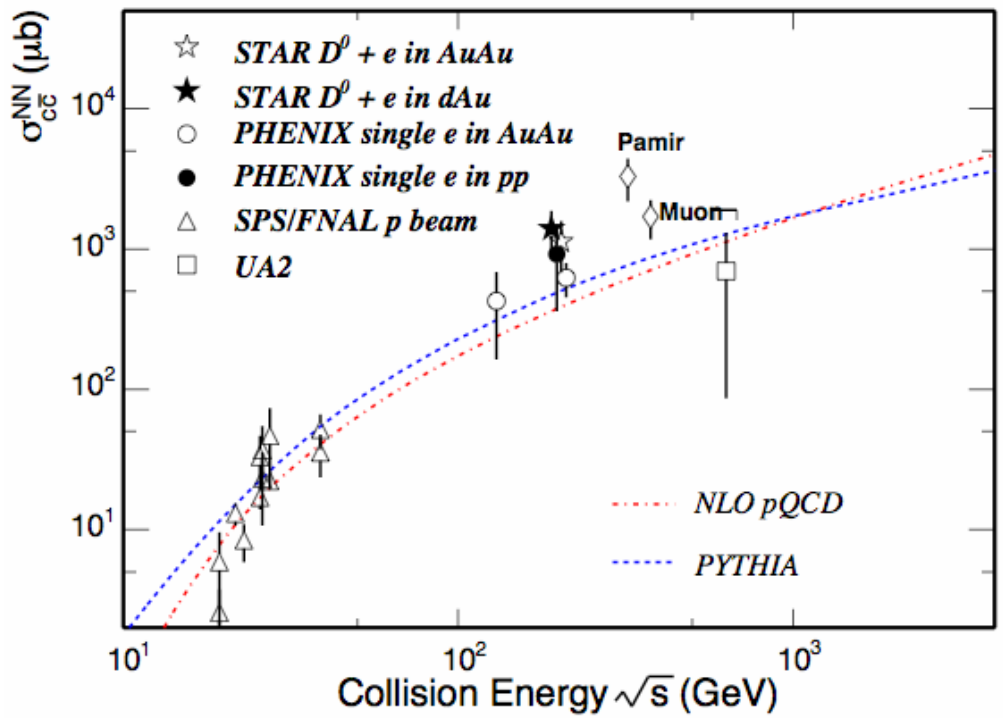

Figure 3: Total cē production cross-sections per nucleon-nucleon collision vs. collision energy. The dashed line depicts a PYTHIA calculation ${ }^{18}$. The dot-dashed line depicts a NLO pQCD calculation with MRST HO, $m_{c}=1.2 \mathrm{GeV} / \mathrm{c}^{2}, \mu_{\mathrm{F}}=2 \mathrm{~m}_{\mathrm{c}}, \mu_{\mathrm{R}}=2 \mathrm{~m}_{\mathrm{c}}{ }^{19}$. The figure is adapted from Ref. [17, 19]. 


\subsection{The Need for Direct Topological Reconstruction of Open Charm}

When direct measurements of heavy flavor hadrons are not possible, non-photonic electrons from heavy flavor decays can be used to study charm production. However, there are serious limitations in such situations. As shown by Batsouli, Kelly, Gyulassy and Nagle in Ref. [20], the decayed electron distributions are insensitive to the intrinsic shape of the D-meson transverse momentum distribution. Due to the decay kinematics and the light mass of the electrons and positrons, the dynamical information in the primary spectrum is washed out. This phenomenon is shown in Figure 4. There is a clear difference between the zero mean free path hydrodynamic flow prediction (solid) and the infinite mean free path pQCD Pythia calculations (dashed-line) for D-mesons; but the resulting electron spectra are nearly indistinguishable. In order to extract useful information about heavy flavor production in heavy ion collisions, we have to measure the charm and beauty-hadrons by direct topological reconstruction. Single electron spectra are not sufficient.

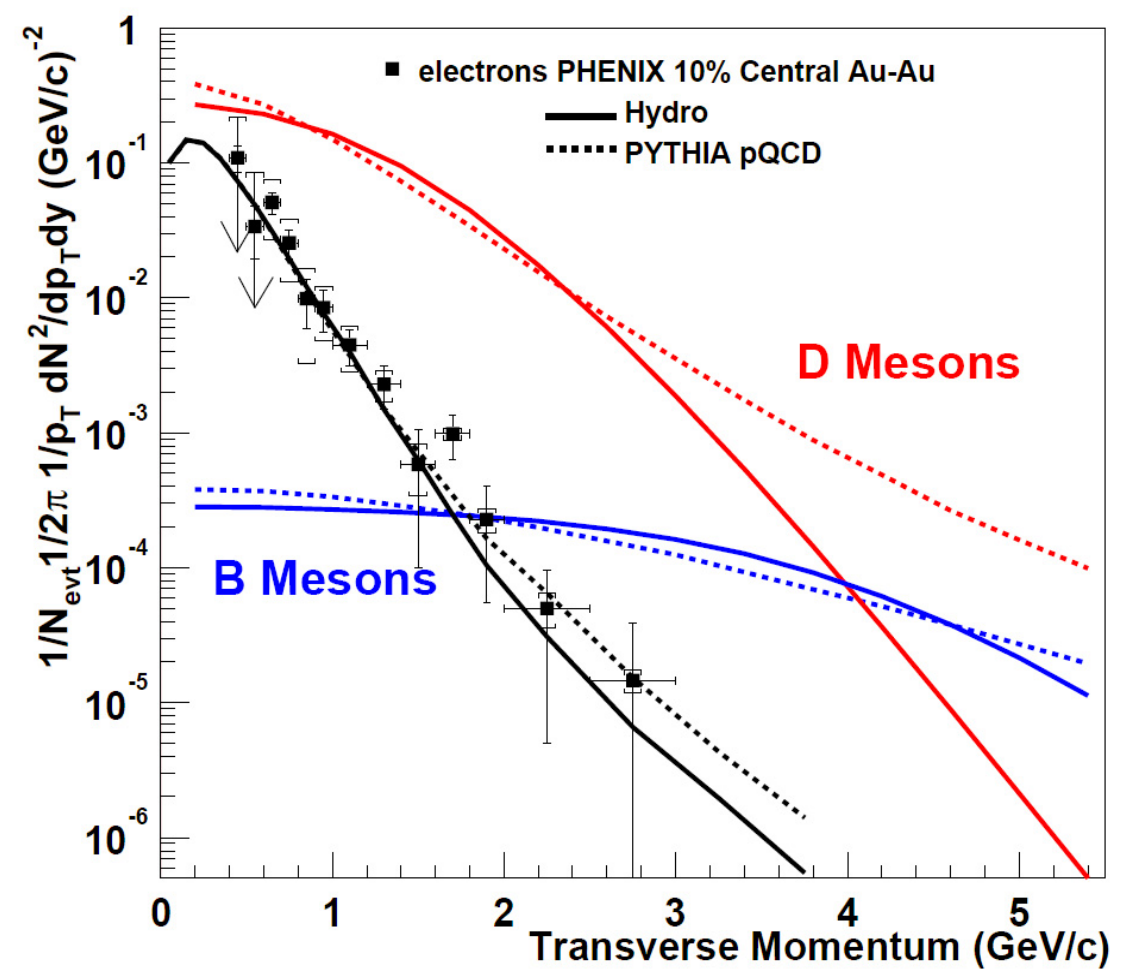

Figure 4: Solid- and dashed-curves represent the charm- (red) and beauty-hadron (blue) spectra from Blast-Wave and Pythia model calculations, respectively. The corresponding heavy flavor decayed electron spectra are shown as black curves. The data are the measured single electron distributions measured in $10 \%$ central Au + Au collisions at $130 \mathrm{GeV}$ by the PHENIX collaboration. The figure is adapted from Ref. [20].

The same conclusion is reached when doing the analysis of $\mathrm{R}_{\mathrm{AA}}$ for heavy flavor spectra. Recall that $\mathrm{R}_{\mathrm{AA}}$ is the ratio scaled by the number of binary collisions of the charm yield measured in heavy ion collisions relative to the yield in $\mathrm{p}-\mathrm{p}$ or $\mathrm{d}$-Au. The nuclear 
modification factor, $\mathrm{R}_{\mathrm{AA}}$, for $\mathrm{D}$-mesons is shown in Figure 5. Two vastly different models are shown. The figure shows that there are large differences in the two D-meson $\mathrm{R}_{\mathrm{AA}}$ ratio curves but the corresponding decayed-electron $\mathrm{R}_{\mathrm{AA}}$ curves are essentially identical for all $\mathrm{p}_{\mathrm{T}}$. These are theoretical curves without error bars which suggests that the decayed electron $\mathrm{R}_{\mathrm{AA}}$ curve is impossible to measure; additionally, any electron measurement will have large systematic uncertainties at low momentum due to the large background from photonic electron production ${ }^{16,17}$. At higher $\mathrm{p}_{\mathrm{T}}$, electrons from B-meson decays will become more abundant making the electron measurement for charm physics even less realistic. This clearly calls for a direct measurement of heavy flavor hadrons, i.e. D- and B-mesons.

The proposed Heavy Flavor Tracker (HFT) will make these and other measurements by the direct topological reconstruction of the various charmed hadrons, including the $\mathrm{D}^{+}$, $\mathrm{D}^{-}, \mathrm{D}^{0}, \mathrm{D}_{\mathrm{s}}^{+}$and possibly $\Lambda_{\mathrm{c}}^{+}$. Thus the HFT will enable us to dramatically reduce the systematic uncertainties that are inherent in single electron spectra.

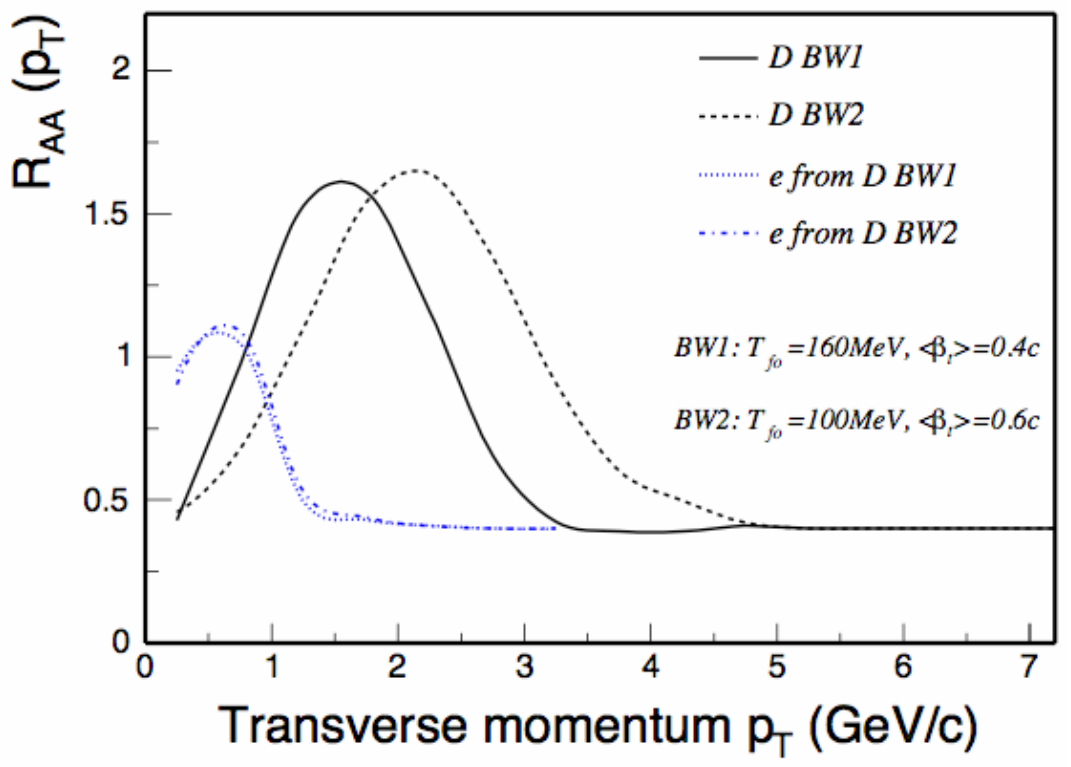

Figure 5: Nuclear modification factor $R_{A A}$ of $D$-mesons assuming a hydro-dynamically inspired parameterization assuming a collective flow velocity of $\left\langle\beta_{\mathrm{r}}\right\rangle=0.4 c$ and $0.6 \mathrm{c}$ for $\mathrm{D}$-mesons. The corresponding electron decay-spectra are shown by the blue dashed (1) and (2) lines ${ }^{21}$.

\subsection{Probing Medium Thermalization: Charm Quark Re-interactions}

RHIC data on light flavor hadrons strongly suggests that partonic collectivity has been achieved in heavy ion collisions. The successful measurement of partonic collectivity is a necessary step toward the discovery of a QGP; however, it is not sufficient information to claim a discovery until we address the issue of thermalization and collectivity, together. 
Collectivity in heavy ion reactions is addressed by studying flow. Many important measurements of transverse radial and elliptic flow of identified hadrons, containing light quark flavors $(q=u, d, s)$, have been performed at RHIC. The main conclusions from these studies are that the systems created in ultra-relativistic $\mathrm{Au}+\mathrm{Au}$ collisions exhibit strong collective expansion. Compared to measurements at lower SPS energies, the degree of collectivity is stronger. In addition, measurements of the spectra of multistrange hadrons ( $\Xi$ and $\Omega$ ) at RHIC suggest that they freeze-out at a higher temperature and lower collective velocity than the lighter hadrons; however, a significant amount of elliptic flow is also observed for multi-strange baryons. The elliptic flow of the multistrange baryons is comparable to the amount of flow observed for the non-strange baryons and is in good accord with the number of constituent quarks (ncq - scaling) hypothesis that describes the non-strange quark bearing mesons and baryons so well ${ }^{22}$. These results have been interpreted as an indication that sizeable partonic collectivity develops at RHIC. Details of these studies can be found in the literature $2,23,24,25,26$.

The key question, then, is whether or not charm quarks flow. If the elliptic flow of charm were comparable to the elliptic flow of the lighter quarks, then this would be a clear indication of a thermalized state of matter at RHIC because it takes many interactions with lighter quarks, and gluons, to cause a heavy quark to acquire the collective motion of the bulk matter.

Theoretical calculations indicate that thermalization may be reached at RHIC at temperatures $\mathrm{T}_{\mathrm{eq}} \approx 0.3-0.5 \mathrm{GeV}^{27}$ and that the duration of the equilibrium period is on the order of 5-10 fm/ $\mathrm{c}^{28,29}$. Thermal production of $c \bar{c}$ pairs is suppressed due to their large masses $\left(\mathrm{m}_{\mathrm{c} \overline{\mathrm{c}}}=2.2-2.3 \mathrm{GeV} / \mathrm{c}^{2}\right)$, however, charmed hadrons may still be produced in a thermalized fashion if the $c$ and $\bar{c}$ quarks become embedded in a thermalized bath of light quarks. The relative probability of creating different charmed hadrons will be driven by the properties of the medium which is providing the additional quarks necessary to form the hadrons. The relative yield of various charmed hadrons is thus sensitive to the properties (temperature and chemical potentials) of the light quark medium and these hadron yields must be measured in order to achieve a full understanding of the medium.

The transverse momentum distributions of these hadrons are particularly important. The $\mathrm{p}_{\mathrm{T}}$ distributions reflect the dynamical evolution of the system and yield indirect information about different stages of the collision. After hadronization is complete and inelastic collisions cease, then the particle abundances are fixed: this is commonly referred to as chemical freeze-out. Later when elastic interactions cease, the particle momentum spectra become fixed; this is referred to as kinetic freeze-out. The evolution of the system is recorded in the $\mathrm{p}_{\mathrm{T}}$ spectra because, for most particle species, transverse radial flow is accumulated throughout the whole collision history while transverse elliptic flow is believed to saturate at early times in the collision sequence. 
In summary, charmed hadron flow is potentially an indicator of thermalization due to the heavy quark interactions with light quarks and gluons. If thermalization takes place, it is reached during the partonic stage of the collision. In order to test the question of thermalization experimentally, we propose to measure the charmed hadron transverse radial and elliptic flow using the STAR HFT detector. The combination of the open charm spectra and $\mathrm{v}_{2}$ will allow us to determine the collective properties of the charm quarks and the probable degree of thermalization of the light quarks.

\subsubsection{Elliptic Flow}

In non-central heavy ion collisions, the overlap of the colliding nuclei is spatially deformed and has an ellipsoidal shape. Through many re-scatterings, the pressure gradients will be built up in non-head on high energy collisions. In this way the initial state spatial anisotropy can be transformed into a final state momentum space anisotropy. Notice that re-scattering is a sufficient condition for the development of these anisotropies and thermalization is not required. Theoretically, the largest momentum anisotropies are obtained in the hydrodynamic limit ${ }^{30}$ where there is a zero mean free path; this leads to instantaneous local thermal equilibrium.

These momentum space anisotropies lead to a dependence of the transverse-momentum distribution on the emission angle relative to the reaction plane. The anisotropy can be quantified by the coefficients of a Fourier decomposition of the distribution in azimuth. The largest contribution comes from the second Fourier coefficient $\mathrm{v}_{2}\left(p_{\mathrm{T}}, y\right)$, the elliptic flow coefficient. In the evolution of elliptic flow, the strong and early spatial deformation decreases because the matter begins to expand more rapidly in the direction of the shorter axis of the ellipsoid ${ }^{31}$. As the spatial deformation disappears, the build-up of flow due to pressure gradients ceases and the elliptic flow saturates. For this reason, elliptic flow is thought to be a signal that develops in the early stages of a collision. RHIC data ${ }^{2,32,33}$ show that in semi-central $\mathrm{Au}+\mathrm{Au}$ collisions, elliptic flow reaches the hydrodynamic limit for transverse momenta up to $2 \mathrm{GeV} / \mathrm{c}$ and this suggests early thermalization ${ }^{30}$ at a time of $\tau=0.6 \mathrm{fm} / \mathrm{c}$ since the collision began. Thus, information about the equation of state $^{34}$ can be determined. If all hadron species experience the same anisotropic flow, their $v_{2}$ coefficients should obey simple hydrodynamic relations ${ }^{34}$ and exhibit a characteristic mass dependence. As an example, Figure 6 (top) shows the measured elliptic flow of strange hadrons up to, and including, the multiply strange $\Xi$ baryon. At low momentum, all particles exhibit a linear rise in $v_{2}$ and a clear mass ordering appears from the lower mass kaon to the heavier $\Xi$. The bottom plot in Figure 6 shows the measured elliptic flow versus transverse momentum; where both axes are scaled with the number of constituent quarks. Quark coalescence models predict a universal scaling of $v_{2} / \mathrm{n}$ versus $p_{\mathrm{T}} / \mathrm{n}$ at intermediate momentum where quark recombination is (supposedly) the dominant hadron production mechanism. In these models, collectivity - the elliptic 
flow of constituent quarks - is intrinsically built in and supports the idea of partonic collectivity. These analyses demonstrate that collectivity is established at the partonic level.
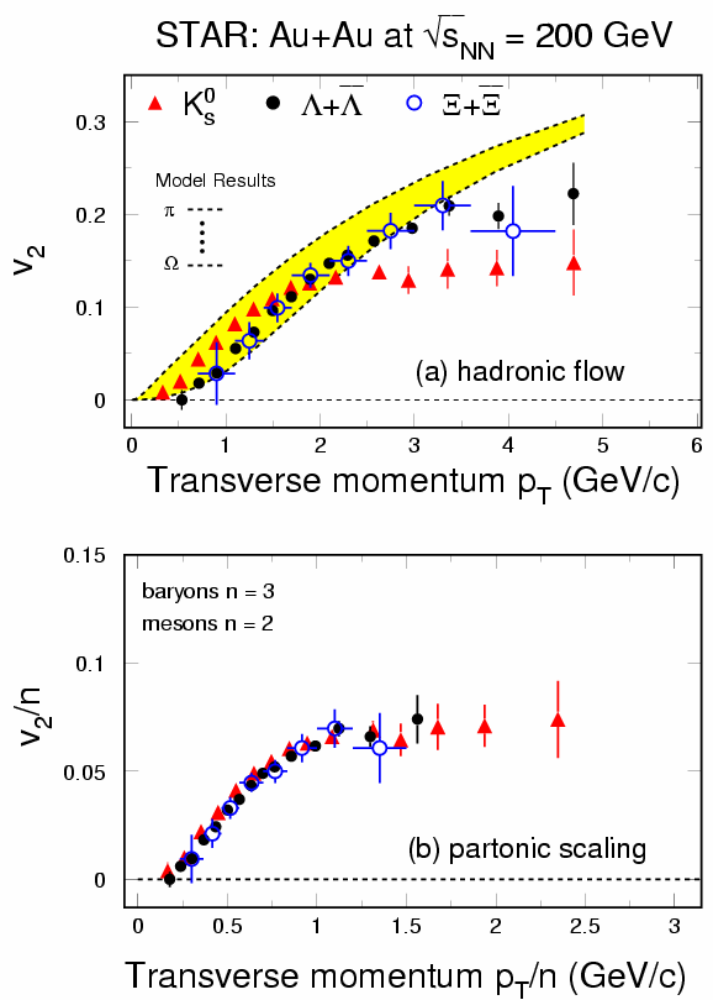

Figure 6: Elliptic flow of strange hadrons at RHIC as measured by the STAR detector. The top panel demonstrates typical hydro-dynamical mass ordering up to a momentum $\mathrm{p}_{\mathrm{T}}<2 \mathrm{GeV} / \mathrm{c}$ and saturation at larger momentum. The bottom panel shows the scaling of elliptic flow with the number $n$ of constituent quarks in the saturation region (baryons, $n=3$; mesons, $n=2$ ).

At RHIC energies, charm quarks are abundantly produced. Due to their high mass and small interaction cross section, the strength of elliptic flow of heavy flavor hadrons may be a good indicator of thermalization occurring at the partonic level. If heavy flavor hadrons flow together with the light flavor hadrons, this indicates frequent interactions between the light and heavy quarks. Hence, thermalization of light quarks is likely to have been reached through partonic re-scattering.

Figure 7 shows the first indication of charm particle elliptic flow at RHIC measured in the inclusive electron channel ${ }^{35}$. The predictions assume elliptic flow for the light quarks as determined by fits to experimental data. Presently, the data support the idea that the heavy charm quarks flow. As shown in the figure, the uncertainties are rather large especially at low transverse momentum where hydrodynamic behavior should occur. A precise measurement of directly reconstructed open charm hadrons to low momentum is essential to confirm and further quantify elliptic flow of the charm quarks. 


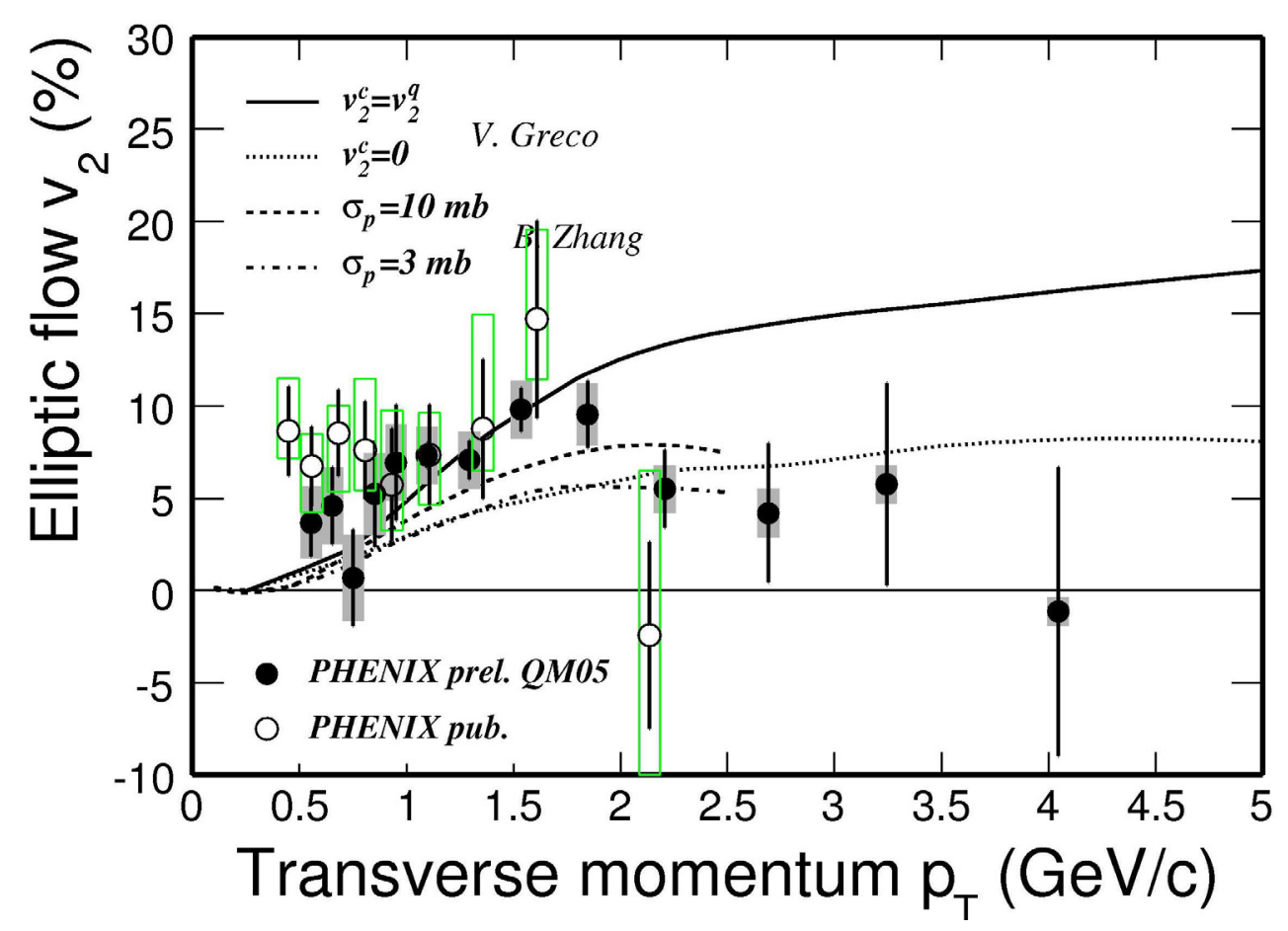

Figure 7: Non-photonic electron elliptic flow at RHIC. Open and closed symbols are data of nonphotonic electron $v_{2}$ from PHENIX ${ }^{35}$ Statistical errors are shown as solid lines and the open boxes

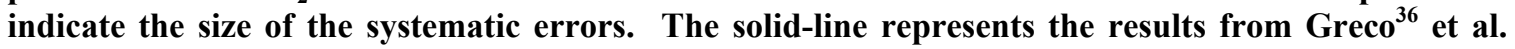
where, in this coalescence calculation, similar $v_{2}$ distributions for c- and $u$-quarks are assumed.

\subsubsection{Charm Hadro-Chemistry}

Hadronic yields and their ratios have been measured from AGS to RHIC energies and have been successfully described by statistical models ${ }^{37,38}$. The relevant parameters are the chemical freeze-out temperature $T_{c h}$, and the chemical potentials $\mu_{i}$ for conserved quantum numbers, i.e. net strangeness, charge, and baryon number conservation ${ }^{39}$. The extracted chemical freeze-out temperature $\mathrm{T}_{c h}$ is higher than the kinetic freeze-out temperature $T_{f o}$ extracted from momentum spectra ${ }^{40}$ and this suggests that chemical freeze-out occurs before kinetic freeze-out, as expected.

Charm quarks are dominantly produced in initial parton-parton scatterings ${ }^{19}$ and the thermal production of charm quarks is suppressed due to their large mass. In the case of sufficient re-scatterings, initially produced charm quarks might thermally (but not chemically) equilibrate with the surrounding medium. This means their momentum distribution can be described by a temperature parameter consistent with the spectra of light quarks, while the total abundance of charm quarks is determined by the initial parton collision dynamics.

These arguments lead to the idea of statistical hadronization of charm quarks ${ }^{41,42}$. Statistical coalescence scenarios predict large changes in open (regarding relative 
abundances, e.g. the $\mathrm{D}^{0} / \mathrm{D}_{\mathrm{s}}{ }^{+}$ratio) and hidden charm production with a strong centrality dependence $^{43}$. Statistical coalescence implies that charm quarks travel over significant distances, e.g. in a QGP. Therefore, we believe that a consistent description of precision data by these models would be an indication of deconfinement ${ }^{43}$.

Measuring the total charm and bottom yields requires measuring the yields of several different hadrons. Indeed, charm quarks may fragment into a variety of hadrons as shown in the first column of Table 2. These fragmentation ratios have recently been compiled ${ }^{44,45}$ using $e-p$ and $e^{+}-e^{-}$collision data. The ratios are found to be independent of the collision energy and the collision system (e-p or $\mathrm{e}^{+}-\mathrm{e}^{-}$). They are likely to be the same in p-p collisions at mid-rapidity where the HFT can be used to detect charmed particles at RHIC. Note that the ratios calculated using PYTHIA ${ }^{46}$, shown in the third column of Table 2, differ significantly from the measured ratios. To avoid any uncertainties in the charge yield measurement, these ratios will have to be measured in $p+p$ collisions at RHIC energies.

\begin{tabular}{|c|c|c|c|}
\hline & $\begin{array}{c}e-p \text { and } e^{+}-e^{-} \\
\text {average }\end{array}$ & Pythia & $\begin{array}{c}\text { Statistical } \\
\text { coalescence }\end{array}$ \\
\hline$f\left(\mathrm{c} \rightarrow D^{+}\right)$ & 0.232 & 0.162 & 0.21 \\
\hline$f\left(\mathrm{c} \rightarrow D^{0}\right)$ & 0.549 & 0.639 & 0.483 \\
\hline$f\left(\mathrm{c} \rightarrow D_{s}^{+}\right)$ & 0.101 & 0.125 & 0.182 \\
\hline$f\left(\mathrm{c} \rightarrow \Lambda_{\mathrm{c}}^{+}\right)$ & 0.076 & 0.066 & 0.080 \\
\hline$f(\mathrm{c} \rightarrow \mathrm{J} / \psi)$ & & 0.006 & 0.057 \\
\hline
\end{tabular}

Table 2: Charm quark fragmentation functions. The left column is from Ref. [44,45]. The right column is from Ref. [43]. The $D^{+}$and $D^{0}$ yields include feed-down from $D^{*+}$ and $D^{* 0}$ decays.

The effect of statistical coalescence is shown in the last column of Table 2 (see Ref. 43). The calculation assumes that charm quarks statistically coalesce with the lighter quarks, (i.e. according to the temperature and chemical potential of the light-quark system) so the temperature and chemical potential are set so that the light hadron yields measured at RHIC are reproduced. In addition, the number of charm quarks present in the system is set by pQCD calculations and this is reflected by a charm-chemical potential in the statistical coalescence model. The results of the model show that statistical coalescence increases the yield of the $\mathrm{D}_{\mathrm{s}}{ }^{+}$meson by $80 \%$ and the $\mathrm{J} / \psi$ yield by a factor of 10 compared to PYTHIA while the yield of $\mathrm{D}^{0}$ and $\mathrm{D}^{+}$decrease slightly. Thus, the ratios $\mathrm{D}_{\mathrm{s}}^{+} / \mathrm{D}^{0}$, $\mathrm{D}_{\mathrm{s}}^{+} / \mathrm{D}^{+}$and $\mathrm{J} / \psi / \mathrm{D}^{0}$ are very sensitive probes of thermal charm hadron production.

Experimentally, we will be able to precisely measure the ratio of $\mathrm{D}_{\mathrm{s}}^{+}$to $\mathrm{D}^{+}$yields because most of the systematic errors in the individual spectra cancel out when they are reconstructed in very similar decay channels: $\mathrm{D}^{+} \rightarrow \mathrm{K}^{-} \pi^{+} \pi^{+}$and $\mathrm{D}_{s}^{+} \rightarrow \mathrm{K}^{-} \pi^{+} \mathrm{K}^{+}$. 
A precise measurement of the total charm production (mostly carried by open charm mesons) will also serve as a baseline for $\mathrm{J} / \psi$ enhancement/suppression measurements, which have been suggested as a possible signature for QGP formation ${ }^{47}$.

\subsection{Probing the Density of the Medium: Heavy Quark Energy Loss}

In order to develop collective flow in heavy ion collisions, there must be frequent interactions between the constituents of the medium. These interactions will cause energy loss for the energetic partons that are traversing the medium and the amount of energy loss will depend on the distance traveled in the medium.

Results reported at the recent QM05 conference on the nuclear modification factor $\mathrm{R}_{\mathrm{AA}}$ indicate that the rate of energy loss for heavy-quarks in central $\mathrm{Au}+\mathrm{Au}$ collisions is surprisingly similar to that for the light-quarks $(u, d, s)$. This experimental observation contradicts our early understanding of the pQCD interactions of energetic partons in a hot and dense medium where much less energy loss was expected for heavy-quarks compared to the light ones ${ }^{48,49,50}$. Since there have been no directly reconstructed heavyquark hadron distributions from RHIC experiments so far, non-photonic electrons in the transverse momentum range $4<\mathrm{p}_{\mathrm{T}}<10 \mathrm{GeV} / \mathrm{c}$ were used for these heavy-quark studies. When analyzing the data with only non-photonic electron $\mathrm{p}_{\mathrm{T}}$ distributions, the data are complicated by an unknown mixture of charm and beauty contributions. Therefore, the only way to disentangle these effects is by a direct topological reconstruction of charmed-hadron distributions and a measurement of charmed hadron angular correlations at RHIC.

\subsection{1 $R_{\mathrm{AA}}$ and Energy Loss}

The discovery of a factor of 4 to 5 suppression of high $p_{T}$ hadrons $\left(5<p_{T}<10 \mathrm{GeV} / \mathrm{c}\right)$ produced in $\mathrm{Au}+\mathrm{Au}$ collisions at RHIC and the disappearance of the away-side jet has been interpreted as evidence for jet quenching ${ }^{3,51,52,53}$. This effect was predicted to occur due to radiative energy loss of high energy partons that propagated through a dense and strongly interacting medium ${ }^{54}$. Heavy quarks are also predicted to lose energy as they traverse the medium, however, their energy loss is predicted to be significantly less because of a suppression of gluon radiation at angles $\Theta<M_{Q} / E$; where $\mathrm{M}_{\mathrm{Q}}$ is the heavy quark mass and $E$ is the heavy quark energy ${ }^{55}$. This kinematic effect is known as the "dead cone" effect. The suppression of small angle radiation has the advantage that the heavy quark fragmentation function and the spectrum of light particles produced in association with the heavy quarks can be calculated perturbatively.

Figure 8 shows the result for the ratio of charm $(\mathrm{H})$ to light (L) quark suppression from QCD calculations assuming a size of about $5 \mathrm{fm}$ for the static medium traversed by the fast quark. For transverse momentum $\mathrm{p}_{\mathrm{T}}>7.5 \mathrm{GeV} / \mathrm{c}$ this ratio is predicted to be about 
2.0 due to the smaller energy loss of the heavy quark. In the case where heavy quarks suffer the same amount of energy loss as light quarks, this ratio would be 1.0. This ratio is exponentially sensitive to the density of color charges in the medium, and so the measurement of nuclear modification factors of open charm mesons at large $\mathrm{p}_{\mathrm{T}}$ is a promising tool for the study and further characterization of QCD matter at RHIC.

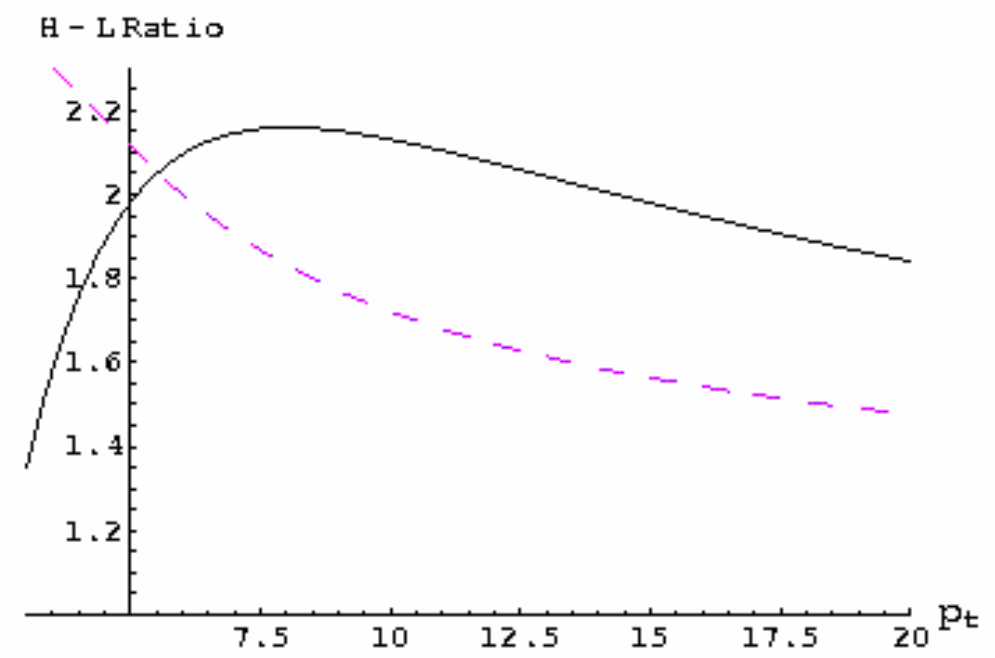

Figure 8: The ratio of suppression factors in hot matter for charm (H) and light (L) quarks. The solid line represents results from calculations with unrestricted gluon radiation, while the dashed line is based on calculations with a cut on gluon energies $\omega>0.5 \mathrm{GeV}$. The size of the static medium traversed by the fast quark is assumed to be $5 \mathrm{fm}$. The figure is from Ref. [49].

Figure 9 shows $\mathrm{R}_{\mathrm{AA}}$ for non-photonic electrons ${ }^{56,57,58}$ from STAR and PHENIX. The data extend up to $\mathrm{p}_{\mathrm{T}} \sim 10 \mathrm{GeV} / \mathrm{c}$. The figure shows that the suppression factor for single electron spectra is in the range of 0.2-0.3 which is almost exactly the same as the suppression factor observed for charged hadrons and pions ${ }^{59,60}$ (i.e. light quarks). Using an unrealistically large initial gluon density of $\mathrm{dN}_{\mathrm{g}} / \mathrm{dy}=3500$, Djordjevic, Gyulassy, Vogt and Wicks have done a pQCD calculation ${ }^{61}$, including gluon radiative energy-loss, which can barely reproduce the electron $\mathrm{R}_{\mathrm{AA}}$ (see the blue-line in Figure 9). The model ignores the contributions from beauty-hadrons, and when beauty is added to the model, the resulting prediction for $\mathrm{R}_{\mathrm{AA}}$ cannot describe the data at all (see the yellow band in Figure 9 ); the model with beauty over-predicts the data by a factor of 2-3. Note that for light-quark hadrons, like pions, a gluon density of $\sim 1000$ has been used to reproduce the observed $\mathrm{R}_{\mathrm{AA}}$. These results raise a serious challenge to our understanding of both the mechanism for heavy-quark production and the mechanism for energy-loss in a hot and dense medium. In order to resolve these important issues, we have to do direct topological reconstruction of open charm. It is experimentally and theoretically too difficult to make definite conclusions from the single-electron measurements due to the complications from the mixing of the electrons from D-meson decay and electrons from bottom hadron decay as well as the smearing of the decay kinematics. 


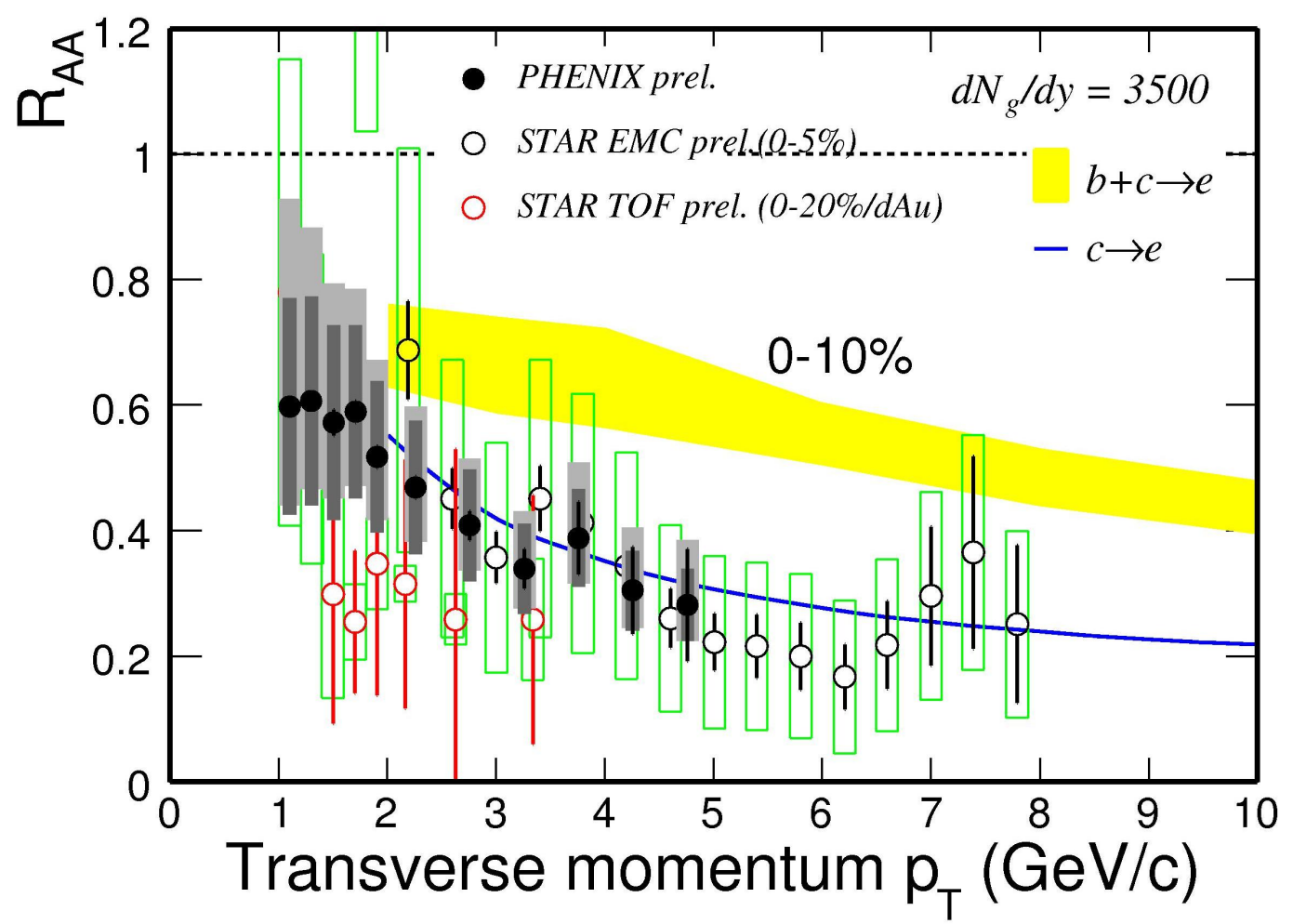

Figure 9: Non-photonic electron $\mathbf{R}_{\mathrm{AA}}$ in central $\mathrm{Au}+\mathrm{Au}$ collisions from STAR (open-symbols, top $5 \%$ ) and PHENIX (filled-symbols, top 10\%) from Refs. [56,57,58]. Theoretical predictions for charmed-hadron and charm and beauty-hadron decayed electrons are shown as blue- and yellowbands, respectively. In these calculations, the initial gluon density was assumed to be unrealistically large: $\sim 3500$, see Ref [61] .

\subsubsection{Charm Angular Correlations}

Correlations between charmed hadrons are another way to separate charm and beauty physics at RHIC. Let the correlation between D mesons be defined as the normalized pair distribution $\mathrm{C}(\Delta \phi)=\mathrm{N}\left(\mathrm{p}_{1}, \mathrm{p}_{2}\right) / \mathrm{N}_{0}$ where $\mathrm{p}_{1}$ and $\mathrm{p}_{2}$ are the momenta of the charmed hadrons, and $\mathrm{N}_{0}$ is the total number of pairs.

Similar to jet production, heavy-quark production requires a large momentum transfer and so we expect a distinct back-to-back topology for the quarks (and mesons) as shown by the open-circles in Figure 10. In this calculation, the Pythia (v6.2) event generator was used with the default sets of parameters. As one can see in the figure, there is a clear back-to-back correlation for the D-mesons. We propose to utilize this distinct correlation to study the charm-quark energy loss in high-energy nuclear collisions. 


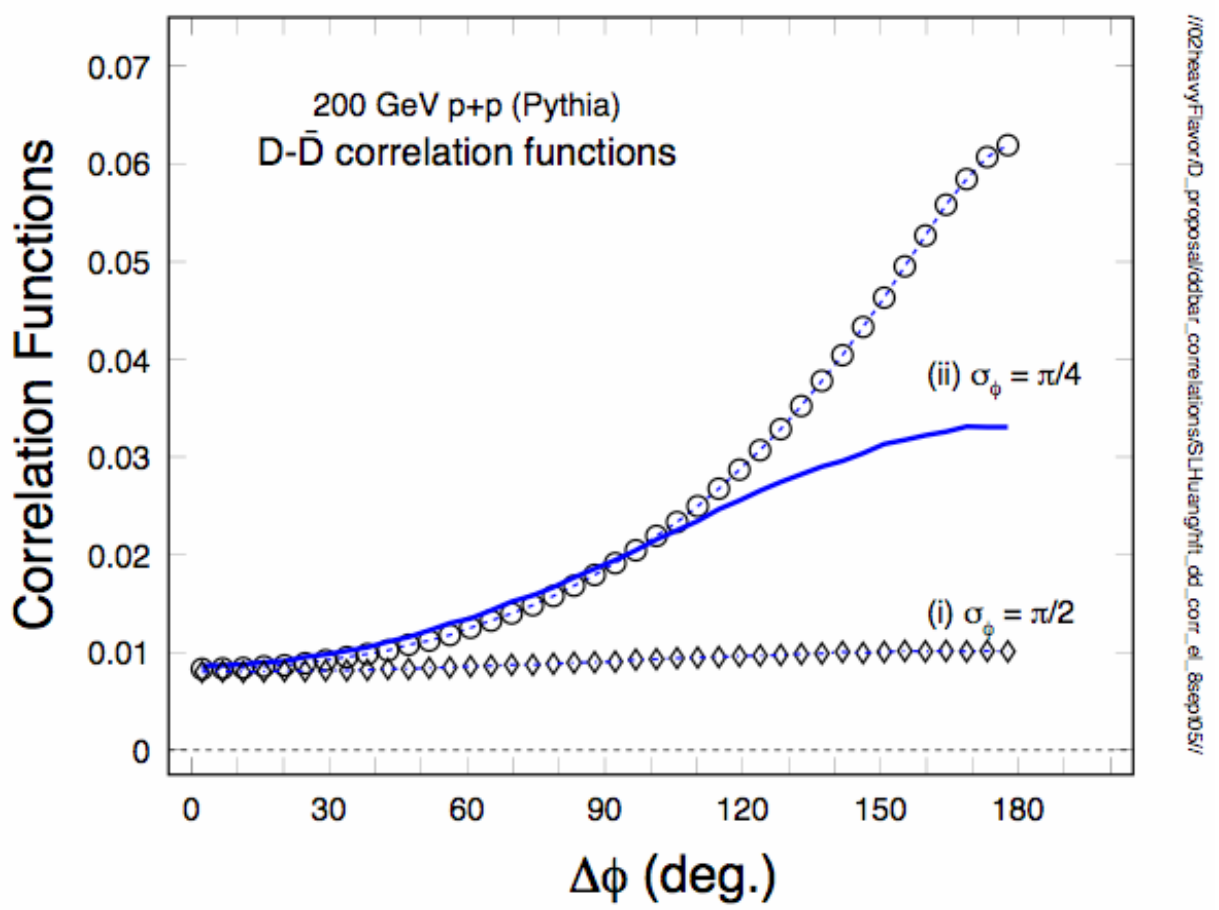

Figure 10: D-meson correlation functions for $200 \mathrm{GeV} \mathrm{p}+p$ collisions. Default parameters in the Pythia model were used in these calculations. A clear back-to-back correlation in the angular distribution of charmed mesons is observed (shown by the open circles). The Solid-line and the diamonds represent the results with angular smearing for $\sigma_{\phi}=\pi / 4$ and $\pi / 2$, respectively.

Now lets consider a few potential observables. For example, when a charm-quark interacts with the medium, it will lose energy and the original angular correlation pattern, as shown in the figure, will be modified. We have tried angular smearings of $\left\langle\sigma_{\phi}\right\rangle=\pi / 4$ and $\pi / 2$. In addition, the changes in the angular correlation depends on the nature of the interaction. Most of the (semi)elastic scatterings are directed in a narrow cone in the forward direction ${ }^{62}$ while the inelastic scatterings, such as the gluon radiative energy $\operatorname{loss}^{48}$ will lead to a much wider smearing in the final correlation. In the inelastic scattering scenario ${ }^{48,50}$, the energy-loss occurs deep inside the plasma and the final correlation function reflects the hot/dense properties of the medium. On the other hand, the resonant scattering happens near $\mathrm{T}_{\mathrm{c}}{ }^{62}$. Although both scenarios lead to sizable energy-loss, the angular correlations may allow us to distinguish these two different mechanisms in high-energy nuclear collisions. In order to perform the measurement, a large acceptance for the reconstructed charmed hadrons is required. The proposed HFT, plus STAR TPC and TOF, will be necessary for this study.

Recently, the measurements on charm production by the Belle Collaboration ${ }^{63}$ show a

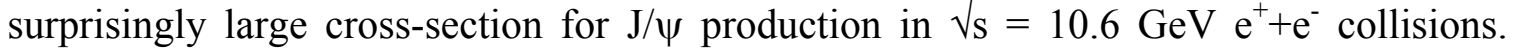
Even more surprising is the fact that more than half of the observed $\mathrm{J} / \psi$ 's are accompanied by c $\overline{\mathrm{c}}$ pairs. This result contradicts our current understanding for $\mathrm{J} / \psi$ 
production in the pQCD framework, such as those discussed in Refs. $[18,64,65,66]$, and implies a different production mechanism for heavy-quarks in elementary collisions ${ }^{67}$. As proposed in Ref. [67], gluon fragmentation is increasingly important for collisions at higher bombarding energies and so RHIC energies are very interesting. In elementary collisions, the main difference between the new and conventional processes lies in the angular correlation of the produced charmed hadrons. With the proposed HFT and STAR EMC (Electro-Magnetic Calorimeter), we will be able to study the correlation of D mesons to further understand $\mathrm{pQCD}$ in $\mathrm{p}+\mathrm{p}$ collisions. We will also study the correlation of D mesons in high-energy nucleus-nucleus collisions, were the gluon density is high ${ }^{68,69}$, and this might enhance the effects observed in elementary $p+p$ collisions. These studies will certainly shed light on the production mechanisms for charm and charmonium at RHIC.

As mentioned earlier, heavy-quark production leads to a back to back correlation between particle and anti-particles. This correlation is also reflected in their decay products, such as the electron pairs. In this case, it causes the background in the intermediate mass region $^{70} 1<\mathrm{m}_{\mathrm{ee}}<3 \mathrm{GeV} / \mathrm{c}^{2}$ to have a correlation, too. (Here $\mathrm{m}_{\mathrm{ee}}$ is the invariant mass of the electron pair.) This creates a significant background for a low mass vector meson analysis. Using the HFT to measure the correlated electron pairs will greatly reduce the background for vector meson and charm measurements via non-photonic electron spectra.

\subsubsection{Baryon - Meson Ratios}

In the intermediate $\mathrm{p}_{\mathrm{T}}$ region $2<\mathrm{p}_{\mathrm{T}}<6 \mathrm{GeV} / \mathrm{c}$, light-quark baryon yields are known to be enhanced compared to meson yields in $\mathrm{Au}+\mathrm{Au}$ collisions at RHIC. The enhancement is found to be proportional to the collision centrality. These observations have been confirmed by both $\mathrm{R}_{\mathrm{AA}}$ and $\mathrm{v}_{2}$ measurements. The results are usually explained by a hadronization mechanism involving collective multi-parton coalescence rather than by independent vacuum fragmentation. The success of the coalescence approach implies deconfinement and possibly thermalization of the light quarks prior to hadronization. Since $\Lambda_{c}$ is the lightest charmed-baryon and its mass is not far from the other D-mesons, it will be very interesting to measure the $\mathrm{R}_{\mathrm{AA}}$ of $\Lambda_{\mathrm{c}}$ and compare it with the $\mathrm{R}_{\mathrm{AA}}$ of the other charmed mesons in order to see if there is a meson-baryon difference. Theories about heavy quark deconfinement and collectivity can be tested with these comparisons. In addition, due to the different branching ratios for the semi-leptonic decays, the measurement of $\Lambda_{\mathrm{c}}$ spectra will help us understand the surprising suppression observed in the non-photonic electrons. In this case, even if charmed quark production scales with the number of binary collisions, an increase in the ratio $\Lambda_{d} / \mathrm{D}$-mesons similar to that seen for the $\Lambda /$ Kaon ratio will lead to a $\sim 20 \%$ suppression in non-photonic electrons $\left(1<\mathrm{p}_{\mathrm{T}}<\right.$ $5 \mathrm{GeV} / \mathrm{c}$ ) for central $\mathrm{Au}+\mathrm{Au}$ collisions ${ }^{71}$. 


\subsection{Measuring Vector Mesons with Dileptons}

In addition to the direct measurement of open-charm hadrons, the HFT will serve as a powerful device to discriminate primordial electrons from background electrons in the measurement of electromagnetic probes.

Photons and leptons emitted from the early stages of heavy ion collisions suffer few interactions with their surroundings since they interact electromagnetically instead of via the strong force. Therefore, electromagnetic probes are ideal tools to study the properties of matter created by relativistic heavy ion collisions.

On the other hand, processes, which produce photons and leptons, are rare and are overwhelmed by photons and leptons from electromagnetic decays of hadrons and subsequent $\gamma$ conversions to leptons. In addition, the final state electrons and positrons are the integrated yield of radiation over the whole evolution of the colliding system. Despite all the experimental difficulties, the low and intermediate mass dileptons have been measured at the CERN-SPS and an excess of radiation above the hadron cocktail has been observed in the invariant mass region of $0.2<\mathrm{M}_{\mathrm{ee}}<0.6 \mathrm{GeV} / \mathrm{c}^{2}$ in semi-central $\mathrm{Pb}+\mathrm{Au}$ collisions ${ }^{72,73}$. Also, new and exciting results from the first three RHIC runs indicate that jets have lost a large fraction of their energy in dense matter and the hadronic phase is relatively short. We believe that these observations at RHIC favor experimental measurements of photons and dileptons due to thermal radiation and will result in a clearer signature than from lower energy heavy ion collisions.

At STAR, electron identification is made possible by a combination of two measurements: a measurement of the energy lost by charged particles due to ionization in the TPC gas $(\mathrm{dE} / \mathrm{dx})$, and a velocity measurement with the time-of-flight system. The relativistic rise of the electron $\mathrm{dE} / \mathrm{dx}$ separates the electron $\mathrm{dE} / \mathrm{dx}$ from those of hadrons except at the crossovers with pions at a momentum of $\sim 0.2 \mathrm{GeV} / \mathrm{c}$, kaons at $\sim 0.6$ $\mathrm{GeV} / \mathrm{c}$, protons at $\sim 1.1 \mathrm{GeV} / \mathrm{c}$ and deuterons at $\sim 1.5 \mathrm{GeV} / \mathrm{c}$. A time-of-flight measurement, using a requirement that $|1-\beta|<0.03$, eliminates slow hadrons and cleans up the crossovers. This results in clean electron identification, as shown in Figure 11. The top plot on the left shows the conventional $\mathrm{dE} / \mathrm{dx}$ measurement in the TPC gas as a function of particle momentum. The bottom plot on the left shows the same $\mathrm{dE} / \mathrm{dx}$ measurement but now requiring $|1-\beta|<0.03$ from the TOF detector. The upper electron band is clearly separated from the lower pion band. Heavier particles (e.g. kaons and protons) are completely removed by the TOF requirement. The right hand plot of Figure 11 shows a $\mathrm{dE} / \mathrm{dx}$ projection in the transverse momentum range $\mathrm{p}_{\mathrm{T}}=1.0-1.5 \mathrm{GeV} / \mathrm{c}$. Even at this rather large momentum, the distribution of electrons is clearly separated from the distribution of charged hadrons. 

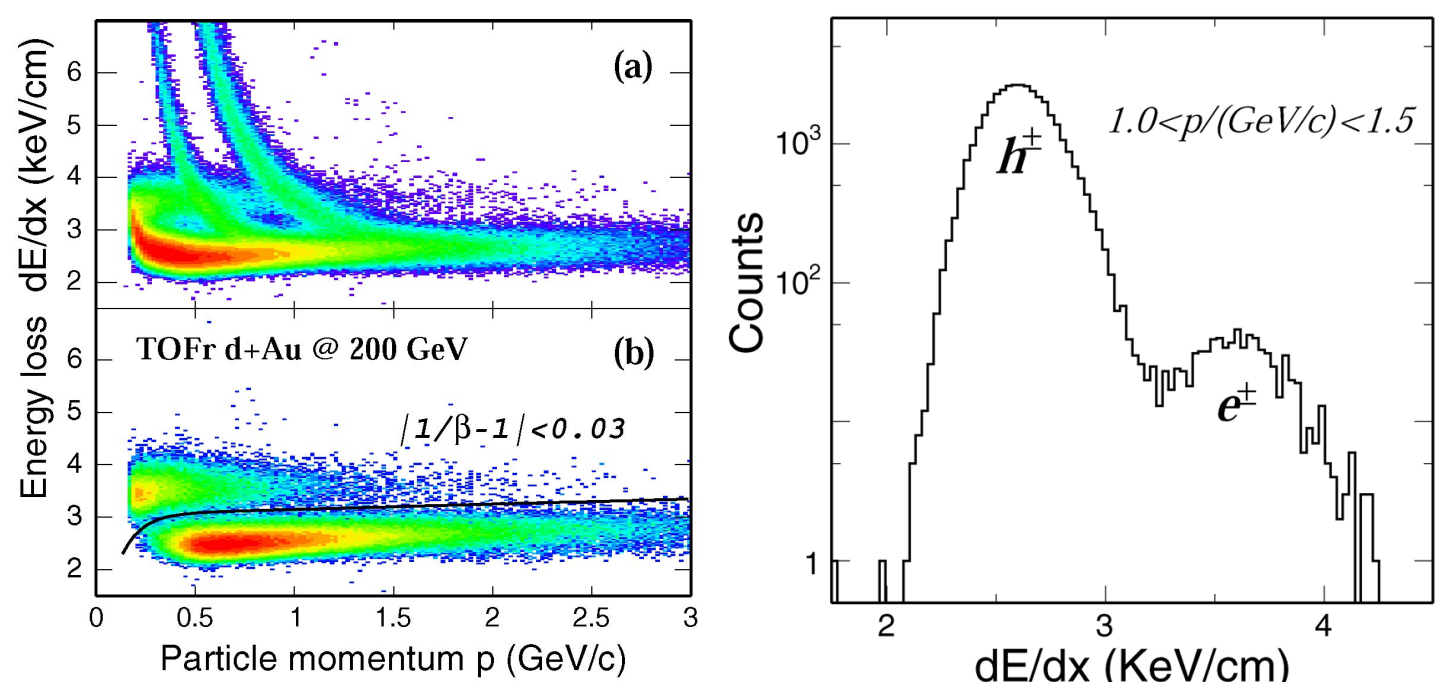

Figure 11: Left top: TPC dE/dx vs. $p$ in $d+$ Au collisions. Left bottom: TPC dE/dx vs. $p$ after TOFr PID selection of $|1-\beta|<0.03$. Clean electron identification is achieved. Right: $d E / d x$ from the TPC after TOFr PID selection for $1.0<p<1.5 \mathrm{GeV} / \mathrm{c}$.

These electrons are, to a large extent, from photons converting into electron-positron pairs $\gamma \rightarrow \mathrm{e}^{+} \mathrm{e}^{-}$in the detector material. The HFT detector will reduce the background of electrons and positrons from these $\gamma$ conversions. By requiring hits in the HFT, electrons from photon conversion outside the HFT, i.e. the SSD, and TPC inner field cage, are rejected. Figure 12 shows the resulting yield from pure Monte Carlo simulations (no tracking involved) of conversion electrons when requiring hits in the TPC only (open circles), hits in the TPC and hits in the TPC+SSD+HFT (closed circles). The requirement of hits in the HFT reduces the detected yield of conversion electrons by a factor of 50 . Full simulations with appropriate pile-up events show that the rate of random ghost tracks is $5 \%$. This means that we can achieve a maximum $\gamma$ rejection by a factor of 20 . To estimate the signal-to-background ratio in the vector meson measurements, we take a reasonably conservative approach and assume that the HFT can reject $\gamma$ conversions by a factor of 10. Another source of electron background comes from the semi-leptonic decays of heavy quark hadrons. The dominant source at intermediate mass ${ }^{74,75}$ is from the semi-leptonic decay of open charm. Due to the large charm yield at RHIC energies, the number of $\mathrm{e}^{+} \mathrm{e}^{-}$pairs from charm-anti-charm decays is comparable to that from $\gamma$ conversion and $\pi$ and $\eta$ Dalitz decays after the HFT rejection factor, described above, is applied. Due to the lifetime of $\sim 100 \mu \mathrm{m}$ of heavy-flavor hadrons, this charm semileptonic decay background can be greatly reduced by measuring the displaced decay vertex with high resolution as can be provided by the HFT detector. Detailed simulations show that HFT is capable of rejecting $75 \%$ of $\mathrm{e}^{+} \mathrm{e}^{-}$pairs from the $\mathrm{D}^{0}$ decay while preserving $50 \%$ of the primordial $\mathrm{e}^{+} \mathrm{e}^{-}$pairs. This increases the signal-to-background ratio by a factor of 2 . 


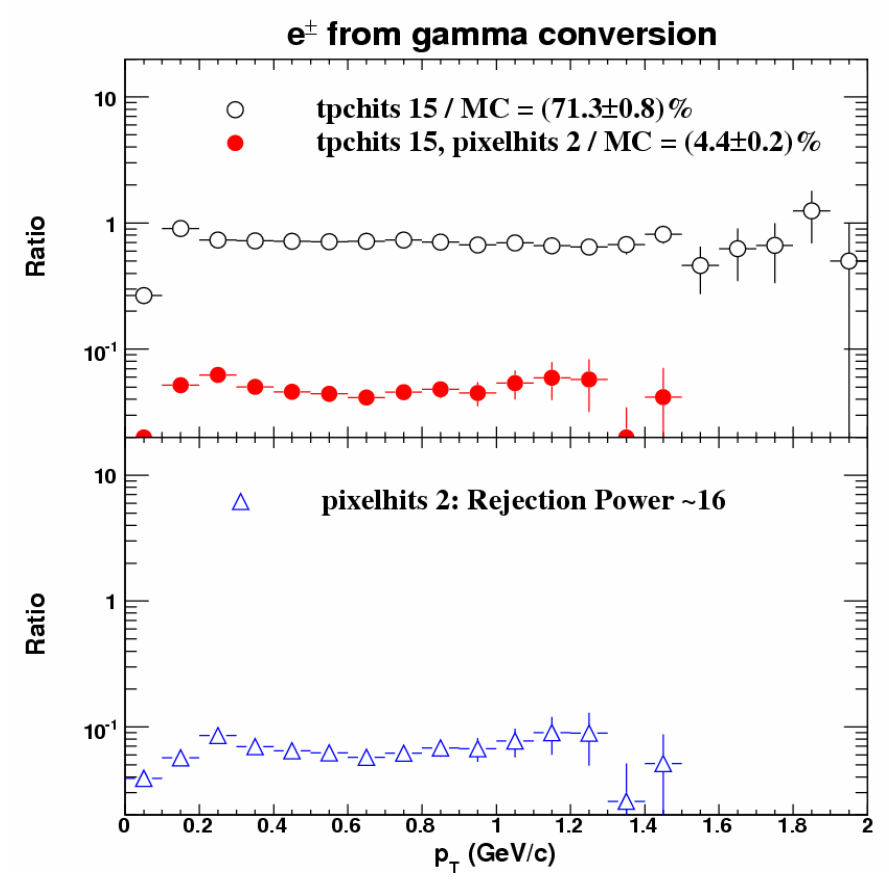

Figure 12: Electron $p_{\mathrm{T}}$ spectra from $\gamma$ conversions reconstructed by requiring TPC tracking alone, a tack in the TPC and 2 HFT hits. The rejection factor is about 20.

The large reduction in photonic-electron background will enable us to observe electromagnetic decays of short-lived vector meson, e.g. $\phi, \omega \rightarrow e^{+} e^{-}$, and intermediate mass dileptons with a few hundred thousand central $\mathrm{Au}+\mathrm{Au}$ events in STAR. The rejection of $\pi^{0}$ and $\eta$ Dalitz decays by a factor of 3 (single track) can be achieved by measuring both electrons, of a pair, which is possible because of the large acceptance of the STAR TPC.

Figure 13 shows the di-electron invariant mass distribution for various signals plus the background. The signals for vector mesons and thermal radiation, are shown as a black curve $^{74}$ and have been calculated using the full acceptance of the STAR detector. The red curve, at the top, is the total di-electron invariant mass yield using the STAR configuration in 2004. This is obtained from single inclusive electron spectrum measurements in $200 \mathrm{Au}+\mathrm{Au}$ collisions with the assumption of electron PID from Full TOF coverage. The gray curve is the $\mathrm{e}^{+} \mathrm{e}^{-}$pair invariant mass distribution for charm after a cut on the HFT DCA $<80 \mu \mathrm{m}$. The dot dashed line show the Dalitz decays from $\pi^{0}$ and $\eta$ after rejection from the TPC. The standard method of dealing with the residual background is by a mixed-event method. This method has been used in CERES and NA52/NA60 at the SPS, and will be used in both PHENIX and STAR. 


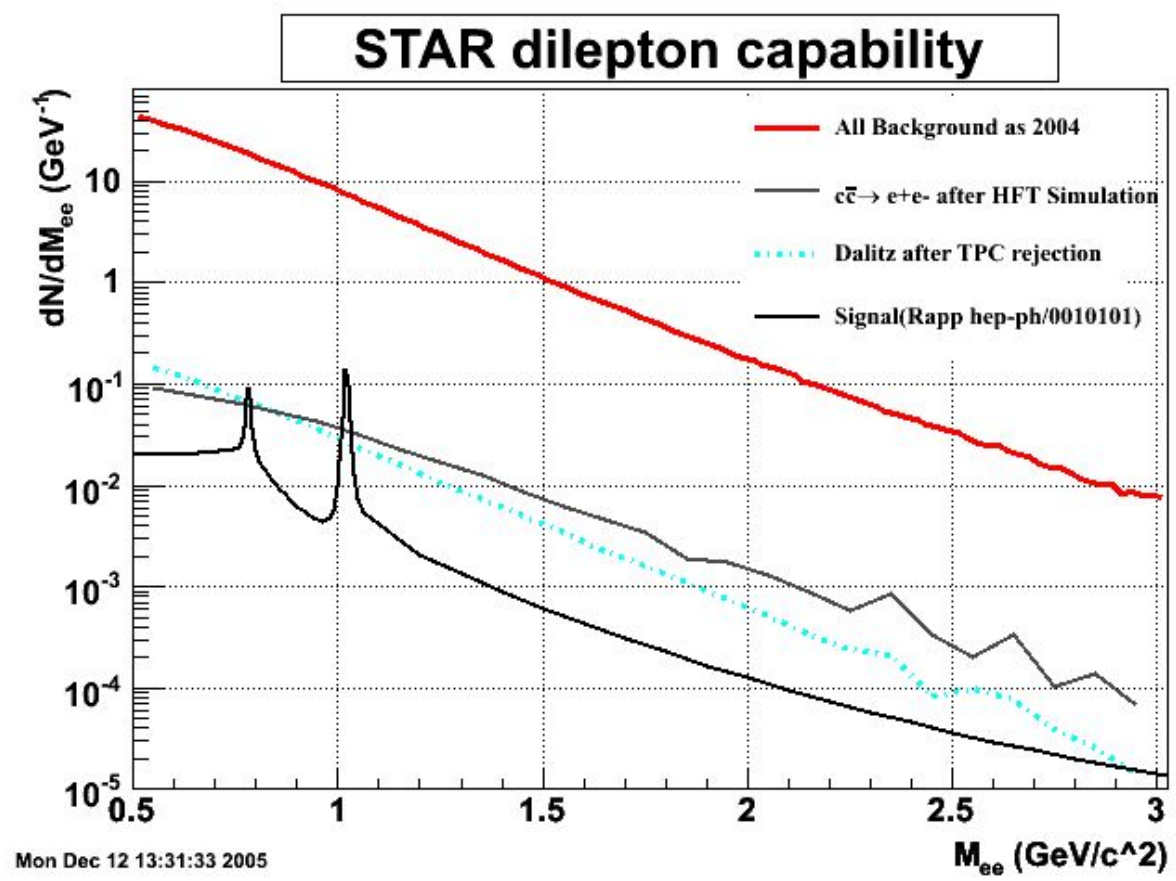

Figure 13: Di-electron invariant mass spectrum. The solid black curve is a prediction from R. Ralf Ref. [74] using the full STAR acceptance. The red curve at the top is the total dielectron invariant mass spectrum seen with the 2004 configuration of STAR but assuming electron PID from Full TOF coverage. The gray curve is the charm e+e- pair invariant mass distribution after a cut on the HFT DCA $<80 \mathrm{um}$. The dot dashed line is the Dalitz decays from $\pi 0$ and $\eta$ after rejection from TPC.

Our simulations show that we can extract a $3 \sigma$ vector meson signal from the data with only $800 \mathrm{~K}$ events in the underlying spectrum. If we had to do this without the HFT, then the same $3 \sigma$ signal would require $8 \mathrm{M}$ events in the underlying spectrum. The inclusion of the SSD and HFT increases the available statistics by a factor of 10. Table 3 shows an estimate of the number of vector meson pairs which can be observed in one RHIC year.

\begin{tabular}{|l|c|c|c|}
\hline Detectors & $\omega$ & $\phi$ & Comments \\
\hline \hline TPC+TOF+SSD+HFT & $22 \mathrm{~K}$ & $6 \mathrm{~K}$ & Pairs per RHIC year \\
\hline
\end{tabular}

Table 3: The number of vector meson pairs which can be recorded by STAR in one RHIC year. We assume $200 \mathrm{M}$ central Au + Au events will be recorded per RHIC year when DAQ 1000 is operational.

For these simulations we assumed a 50\% efficiency for matching tracks between the TPC and the HFT, and we assumed a 50\% efficiency for TOF + TPC tracking and PID. An online trigger was not employed. The results shown in Table 3 results are comparable to the NA60 In + In run results. 


\subsection{Spin Measurements}

The European Muon Collaboration ${ }^{10}$ discovered that the spin of the quarks only contributes a small fraction to the total spin of the proton and this has motivated, and continues to motivate, considerable experimental and theoretical activity to further unravel the mystery of the nucleon spin structure. At present, only the total quark spin contribution is known with reasonably good precision. The most prominent unknown is the spin-dependent gluon density, $\Delta \mathrm{G}$.

The main goal of the RHIC spin program ${ }^{76}$ is to precisely determine $\Delta \mathrm{G}$ by measuring double spin asymmetries in longitudinally polarized proton-proton collisions for a series of complementary reaction channels and over a broad range of the gluon momentum fraction $\mathrm{x}_{\mathrm{g}}$ and transverse momenta $\mathrm{p}_{\mathrm{T}}$. One of the promising reaction channels in STAR is the production of prompt photons and jets with high transverse momenta.

The production of charm and bottom quarks proceeds, in leading order in the strong coupling constant $\alpha_{s}$, through gluon-gluon fusion and quark anti-quark annihilation. The gluon-gluon fusion process is known to be dominant in the spin averaged case for all experimentally relevant kinematic regions. In the spin dependent case both processes have large analyzing powers, as measured by the parton-parton spin asymmetry $a_{L L}$, which approaches -1 for asymptotically large transverse momenta. The masses of the heavy quarks lead to large modifications of the parton-parton spin asymmetry in the kinematic region accessible with $\mathrm{STAR}^{77}$. Next-to-leading order corrections are available and predict relatively sizable changes in the observed asymmetry as compared to leading order expectations ${ }^{78}$.

These properties make charm and bottom production a good test to advance the current understanding of the nucleon spin (unless $\Delta \mathrm{G}$ is found to be vanishingly small).

As is the case for the study of heavy quark energy loss described in this proposal, the relative smallness of the production cross section at large transverse momenta requires triggering with the STAR electromagnetic calorimeters. In spin measurements, the trigger serves to relate the event to the RHIC beam bunch crossing, which determines the spin orientations of the colliding protons. Commonly used parameterizations of the deepinelastic scattering data ${ }^{79}$ lead us to expect measurable, and negative, double longitudinal spin asymmetries at the level of $-5 \times 10^{-3}$ in heavy flavor production in STAR. These asymmetries originate from gluon polarization at intermediate momentum fractions $\mathrm{x}_{\mathrm{g}}$ and are expected to grow in size with increasing $\mathrm{p}_{\mathrm{T}}$ of the decay electron or positron.

In order to make these measurements, fast external pointing with high-rate capability is required to distinguish collisions from different bunch crossings and, hence, different spin orientations. The HFT is an ideal component in this program, however, a high-rate intermediate tracker (IST) is also required. 


\section{HFT Overview and Simulation}

\subsection{STAR and the HFT detector}

$\mathrm{STAR}^{80}$ is one of the two large detectors currently operating at RHIC. It was designed to make measurements of hadron production over a large solid angle, and it features detector systems for high precision tracking, momentum analysis and particle identification (see Figure 14). It is the only experiment at RHIC which measures the full azimuth in $\phi$ out to \pm 1.5 units in rapidity, and tracks particles from $100 \mathrm{MeV} / c$ to 20 $\mathrm{GeV} / c$, so it is well suited for event-by-event characterizations of heavy ion collisions and for the detection of hadron jets.

By adding the Heavy Flavor Tracker (HFT) to STAR, we will be able to measure displaced vertices that are displaced 100 microns, or less, from the primary vertex and thereby identify neutral and charged particles with very short lifetimes can be distinguished from the primary particles which originate at the collision vertex. The addition of the HFT will extend STAR's unique capabilities even further by providing particle identification for hadrons containing charm and beauty and electrons decaying from charm and beauty hadrons. Thus, the HFT is the enabling technology for making direct charm and beauty measurements at STAR.

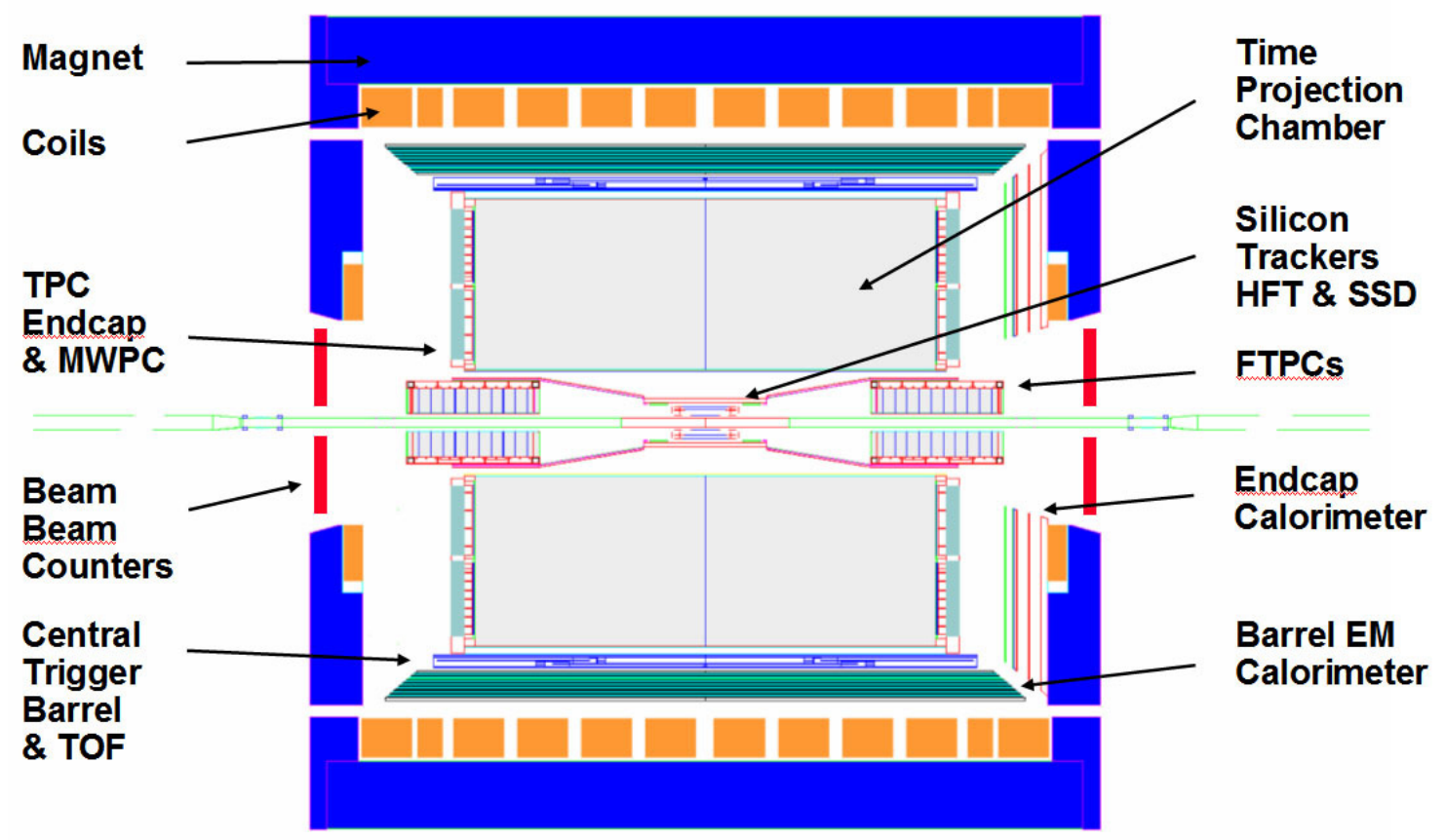

Figure 14: The STAR detector at RHIC. The primary detector elements at mid-rapidity are shown in this cut-away view of the detector. For scale, the TPC is 4.2 meters long and 4 meters in diameter. Heavy Ion beams enter from the left and right while collisions take place in the center of the detector. 
STAR currently has several tracking detectors at midrapidity; we will focus on the Silicon Strip Detector (SSD) and the Time Projection Chamber (TPC). The SSD is installed at a radius of $23 \mathrm{~cm}$, while the TPC tracking begins at a radius of approximately $60 \mathrm{~cm}$. The TPC tracking system extends out to a radius of $190 \mathrm{~cm}$ and yields a total of 45 position measurements along the track of a particle. At the time of HFT installation, a complete Time of Flight detector will surround the TPC barrel $(\phi=2 \pi)$. This detector will replace the Central Trigger Barrel (CTB), which is a simple array of scintillators without TOF information. The new TOF detector will surround the outer field cage of the TPC and will extend the PID region where kaons, pions, and protons can be separated. Also by the time the HFT is installed in STAR, the existing Silicon Vertex Detector (SVT) will be decommissioned and it will be replaced by a new Intermediate Silicon Tracker (IST) that is capable of running at the highest data acquisition rates envisioned for STAR. But before the IST is ready, there will be an opportunity to do significant R\&D with a prototype HFT detector and so the simulations presented in this chapter (unless specified) use only the TPC and the SSD and do not utilize an intermediate tracking device at radii less than $23 \mathrm{~cm}$.

Tracking without an intermediate tracking device, such as the SVT, is not as severe a problem as it may sound. The purpose of the intermediate tracking device is to increase the tracking precision at the outer layer of the HFT, in order to increase the probability of matching the correct HFT hits. It turns out that a tight vertex constraint also enables us to do the track matching with high efficiency. All of our simulations in this chapter are done using a $100 \mu \mathrm{m}$ vertex constraint instead of an intermediate tracker. A vertex resolution of $100 \mu \mathrm{m}$ is easily achieved in a high multiplicity heavy ion collision at RHIC ( $>90$ tracks per event). When the IST becomes available in the future, a vertex constraint will not be necessary and the physics results presented here will only get better; especially for low multiplicity events such as p-p and peripheral collisions. Thus we strongly encourage the collaboration to add a high resolution pointing device between the HFT and the SSD, however, there is a very exciting baseline physics program that we can accomplish with the HFT detector operating in stand-alone mode and so this baseline program is what we will discuss in the remainder of this chapter.

At the present time, the STAR detector has modest capabilities to measure secondary decay vertices that are displaced from the primary vertex. To make these measurements, we use the TPC and the SSD to determine the position of the primary vertex to a precision of approximately $100 \mu \mathrm{m}$ in the central collisions. Thus, by identifying decay vertices that are displaced from the primary vertex, we can identify secondary decays of neutral and charged particles.

For example, is possible to use this technique to identify the $K_{S}^{0}$ because a $K_{S}^{0}$ decays into two pions with a mean lifetime of $0.896 \times 10^{-10}$ seconds and it has a characteristic decay 
distance of $2.68 \mathrm{~cm}$. Since its decay distance is so much greater than the STAR vertex resolution, this particle can easily be identified. Similarly, strange particle decays such as the $\Omega$ baryon can also be identified.

By improving the single track pointing accuracy of STAR by a factor of 50, the HFT allows us to measure very short-lived particles. The HFT will be a low mass detector, and it will be located very close to the beam pipe. It will have two layers of silicon pixel detectors; one at $1.5 \mathrm{~cm}$ average radius and the other at $5.0 \mathrm{~cm}$ average radius. Each layer will be composed of $30 \mu \mathrm{m} \times 30 \mu \mathrm{m}$ pixels and the pointing accuracy of the system (including multiple coulomb scattering in the beam pipe and Si layers) will be better than $60 \mu \mathrm{m}$ for $1 \mathrm{GeV} / \mathrm{c}$ tracks.

\subsection{The Design of the HFT}

The HFT is shown schematically in Figure 15 and its parameters are summarized in Table 4. The innermost layer of the detector lies at a mean radius of $1.5 \mathrm{~cm}$ radius and it is composed of 6 detector ladders. The outer layer lies at a mean radius of $5.0 \mathrm{~cm}$ and it is composed of 18 ladders. Each ladder contains a row of 10 monolithic CMOS detector chips and each ladder has an active area of $19.2 \mathrm{~cm} \times 1.92 \mathrm{~cm}$. Each CMOS detector chip contains a $640 \times 640$ array of $30 \mu \mathrm{m}$ square pixels and is thinned down to a thickness of $50 \mu \mathrm{m}$ to minimize multiple coulomb scattering (MCS) in the detector. The effective thickness of each ladder is $0.28 \%$ of a radiation length. For the purpose of Monte Carlo simulations, however, we have used an effective thickness of $0.38 \%$, per layer, to allow for the possibility of adding an onboard readout chip.

The HFT will achieve maximum vertex resolution by lying as close as possible to the interaction point without residing inside the RHIC beam pipe. To bring the detector to $1.5 \mathrm{~cm}$ radius will require a new, smaller, beam pipe and this also gives us the opportunity to make the beam pipe thinner $(0.5 \mathrm{~mm})$. The new beam pipe, which will reduce the MCS scattering in front of the HFT, is an essential part of the proposal and its design will be discussed in section 6.6. 

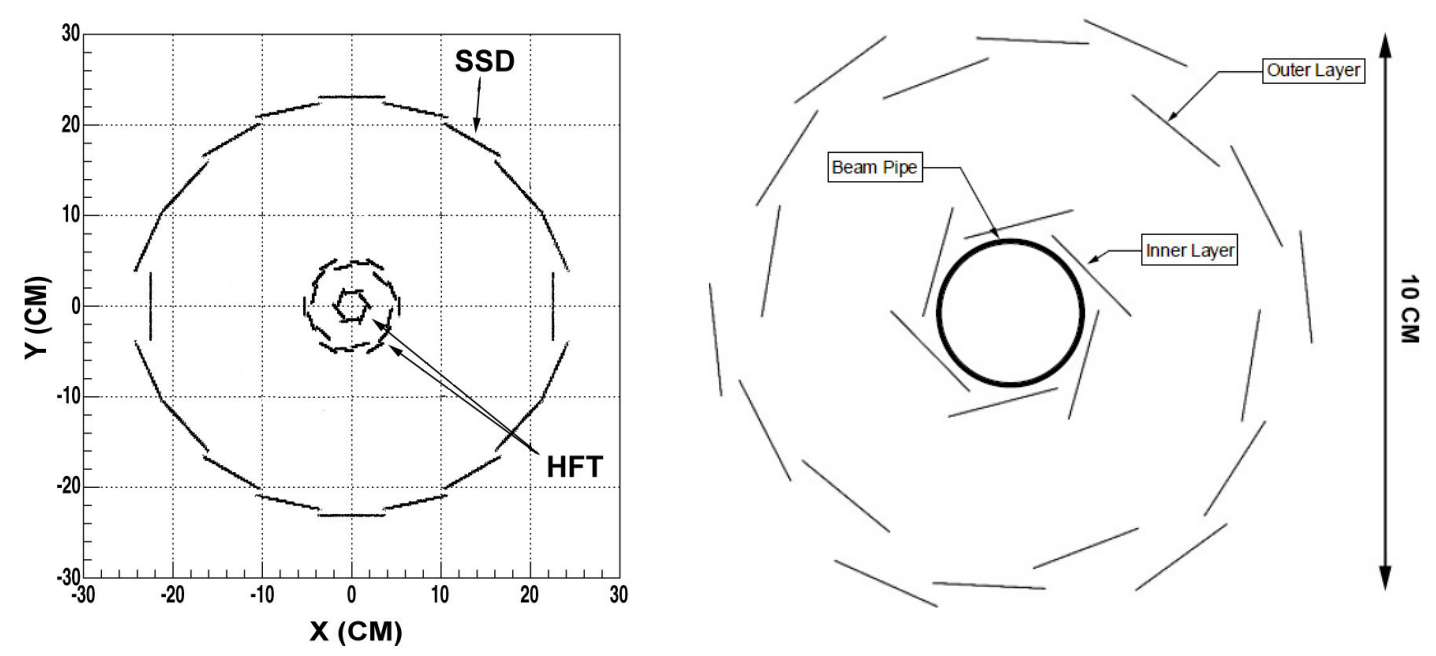

Figure 15: Left - The HFT is shown embedded inside the SSD. Right - Schematic view of the HFT detector ladder arrangement with 6 ladders forming the inner layer and 18 ladders forming the outer layer. The width of the ladders (the dimension seen in this view) is $19 \mathbf{~ m m}$. The shingled layers provide cross calibration of the ladders and provide an open geometry for ease of installation.

\begin{tabular}{|l|l|}
\hline Number of pixels & $98,304,000$ \\
\hline Pixel dimension & $30 \mu \mathrm{m} \times 30 \mu \mathrm{m}$ \\
\hline Detector Chip active area & $19.2 \mathrm{~mm} \times 19.2 \mathrm{~mm}$ \\
\hline Detector Chip pixel array & $640 \times 640$ \\
\hline Number of ladders & 24 \\
\hline Ladder active area & $192 \mathrm{~mm} \times 19.2 \mathrm{~mm}$ \\
\hline Number of barrels & 2 \\
\hline Inner barrel (6 ladders) & $\mathrm{r}=1.5 \mathrm{~cm}$ \\
\hline Outer barrel (18 ladders) & $\mathrm{r}=5.0 \mathrm{~cm}$ \\
\hline Frame read time & $4 \mathrm{~ms}$ \\
\hline Pixel read rate, after zero suppression & $63 \mathrm{MHz}$ \\
\hline Ladder (w/Al cable) \% $\mathrm{X}_{0}$ & $0.28 \%(0.38 \%)$ \\
\hline Beam Pipe Thickness & $0.5 \mathrm{~mm}$ or $0.14 \% \mathrm{X}_{0}$ \\
\hline
\end{tabular}

Table 4: Selected HFT parameters for the detector we propose to build. The simulated parameters are the same except that we have assumed that the detector is $0.38 \%$ radiation lengths thick, per layer, in order to allow for the possibility of an onboard readout chip.

\subsection{Monte Carlo Simulations}

The STAR experiment makes use of an extensive simulation framework to accurately predict the performance of the physical detector. This framework involves many software packages working in tandem to produce the final results. We will give a general overview 
of the simulation chain in order to place the results and predictions contained in this document in a broader context.

The philosophy of the simulation is to use as much standard STAR software as possible. For instance, we use the standard GSTAR simulation package with modifications for the new beam pipe design and the introduction of the HFT. For tracking, we use the new STAR tracking software package ITTF. During the process of writing this proposal, we needed a stable platform, so we froze the ITTF software on February 5, 2005. To that package, we added tracking in the HFT layers. Since ITTF was never optimized for the high precision requirements of the HFT, we have tuned it for this new configuration and added new support software, as required. We will indicate where this new software is used.

The simulation framework has three stages:

1. The simulation of the physics under study: for example a Au-Au interaction at $\sqrt{\mathrm{s}}_{\mathrm{s}}=200 \mathrm{GeV}$.

2. The simulation of produced particles as they interact with the detector and physical structures of the experiment; and finally

3. The simulation and digitization of signals in the detector.

The goal of the simulation software package is to use the same analysis methods on both the simulated and real data, to predict performance and optimize analysis methods.

\subsubsection{Physics Simulation}

The simulations presented in this chapter separate the charmed mesons and baryons from the rest of the event. This has the benefit of greatly increased speed for the analysis of events and for the development of algorithms. This method has been used previously by the ALICE collaboration ${ }^{81}$. For charm production, events with one 'signal' particle were simulated independently from the background. The background for these studies were simulated as a cocktail of pion, kaon, and protons at the maximum multiplicity expected for central collisions, and produced with the MEVSIM package developed for use in $\mathrm{STAR}^{82}$. The 'signal' and 'background' events are analyzed using the same reconstruction and analysis code, with the same cuts and with the same tracking parameters. (For further details see section 3.7, Table 9, and Table 10).

The physical interaction between daughter particles and the material of the detector, as well as signal digitization, are simulated using the STAR implementation of the GEANT simulation package ${ }^{83}$ This package is used in STAR and is a standard analysis tool which includes a detailed understanding of the TPC response function; including dead areas and realistic detector resolutions and responses. 
For both the SSD and HFT detectors, the exact crossing point of the particle through the detector is reported by GEANT to the track reconstruction package. Realistic detector resolutions are used to smear the perfect position information, and the resulting simulated hits are used in tracking. For the SSD, the hits were smeared by $20 \mu \mathrm{m}$ in $\phi$ and $750 \mu \mathrm{m}$ in $z$, in agreement with the SSD specifications ${ }^{84}$. For the HFT, the hits were smeared by $6 \mu \mathrm{m}$ in both $\phi$ and $\mathrm{z}$. We ignore the effects of cluster overlap because we expect these effects to be minimal in the case of the HFT as the occupancy, even in a central $\mathrm{Au}+\mathrm{Au}$ event, is relatively low.

For these simulations, the event reconstruction algorithms traditionally used in STAR were modified to optimize the HFT hit finding accuracy and vertex reconstruction performance. These modifications and performance improvements are detailed in the next section.

\subsubsection{Reconstruction}

Reconstruction of the simulated events is handled by the usual framework implemented in STAR to unite the various tracking detectors. We use this package, along with a detailed GEANT simulation, to provide an estimate of the expected performance of the HFT in the full suite of STAR detectors.

As mentioned the introduction to this chapter, we use a vertex constraint in conjunction with the TPC and SSD detectors to provide efficient reconstruction and track matching purity. In this algorithm, the STAR vertex finder package uses tracks defined with only the TPC and SSD to determine a primary vertex. If no vertex is found for an event, the event is lost. Since the 'signal' events contain one signal particle per event, the Monte Carlo vertex is used, but smeared with a resolution typical for events of these centralities $(100 \mu \mathrm{m})$.

With the current STAR algorithm, the tracker follows a series of hits in the TPC and SSD, refitting after every new hit is added. These tracks (called "global" tracks) are then passed to the vertex finder, which reconstructs the event vertex. The global tracks are extended to the resulting reconstructed vertex, and, if the fit is successful, the new track is stored and labeled a primary track.

The new algorithm simply takes these primary tracks and refits the track, this time with the HFT hits available. HFT hits that meet the tracking selection criteria are added. The Kalman fitter updates the track fit as the new HFT hits are added. The tracks with added HFT hits are then stored See Figure 16 for a block diagram of this algorithm. The success of the algorithm depends on the precision of finding the primary vertex, which is centrality dependent. 


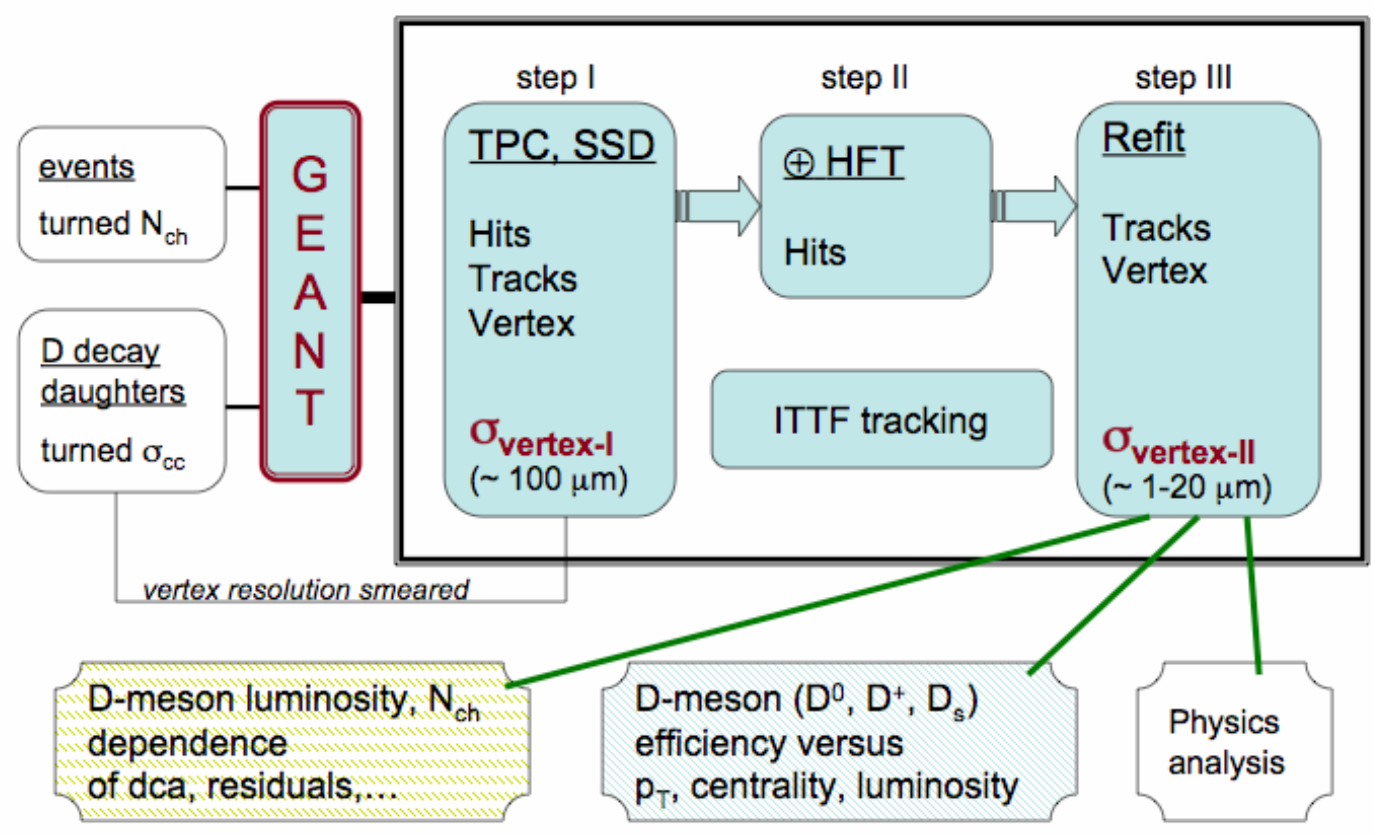

Figure 16: Simulation block flow diagram. Only small modifications were needed to optimize the STAR reconstruction software for use with the HFT.

The dramatic improvement of the track fit at this stage allows a more accurate primary vertex fit. A simplified vertex algorithm was adopted for this refit. The distribution of the distance of closest approach for each HFT track to the TPC + SSD primary vertex shows a distinct offset from zero, as well as a non-gaussian shape (see Figure 17). The offset (calculated in three dimensions) is used as a correction to the primary vertex position. We found that iterating on this procedure produced the best resolution (see Figure 18). A final vertex position resolution of $8 \mu \mathrm{m}$ was achieved for the highest multiplicity events, while in the most general case the vertex resolution follows the functional form:

$$
\sigma=\frac{380 \mu m}{\sqrt{N_{c h}}}
$$

where $\mathrm{N}_{\mathrm{ch}}$ is the multiplicity of charged particles in the detector acceptance. The vertex resolution functional form follows Poisson statistics, as expected. This functional form is drawn as a line to guide the eye in Figure 19. The highlighted region in Figure 19 demarcates the centrality region of TPC + SSD vertex resolution that is useful and allows the tracking code to achieve the necessary hit purity in the HFT. The highlighted region corresponds to $0 \%-60 \%$ centrality in $\mathrm{Au}-\mathrm{Au}$ collisions or, equivalently, to a multiplicity of $\mathrm{N}_{\mathrm{ch}}>90$. 


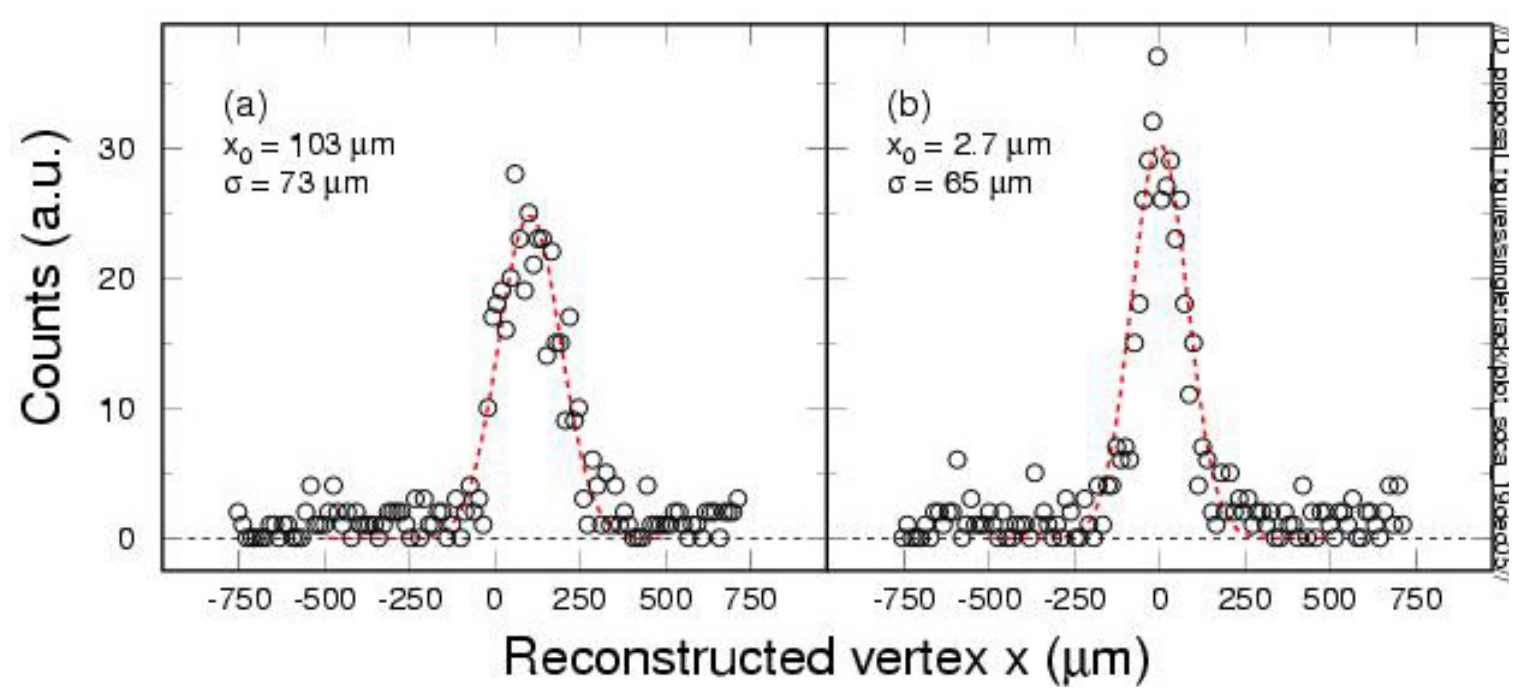

Figure 17: DCA of tracks to the Primary vertex. The geometric signed distance of closest approach between all tracks $\left(p_{\mathrm{T}}>200 \mathrm{MeV} / \mathrm{c}\right)$ and the vertex is shown for two cases: a) DCA to the vertex defined by TPC+SSD tracks. The non-zero mean of this distribution is used to correct the vertex coordinate. b) DCA distribution after iterative correction using TPC+SSD+HFT tracks, with a significant improvement in the mean and shape of the distribution.

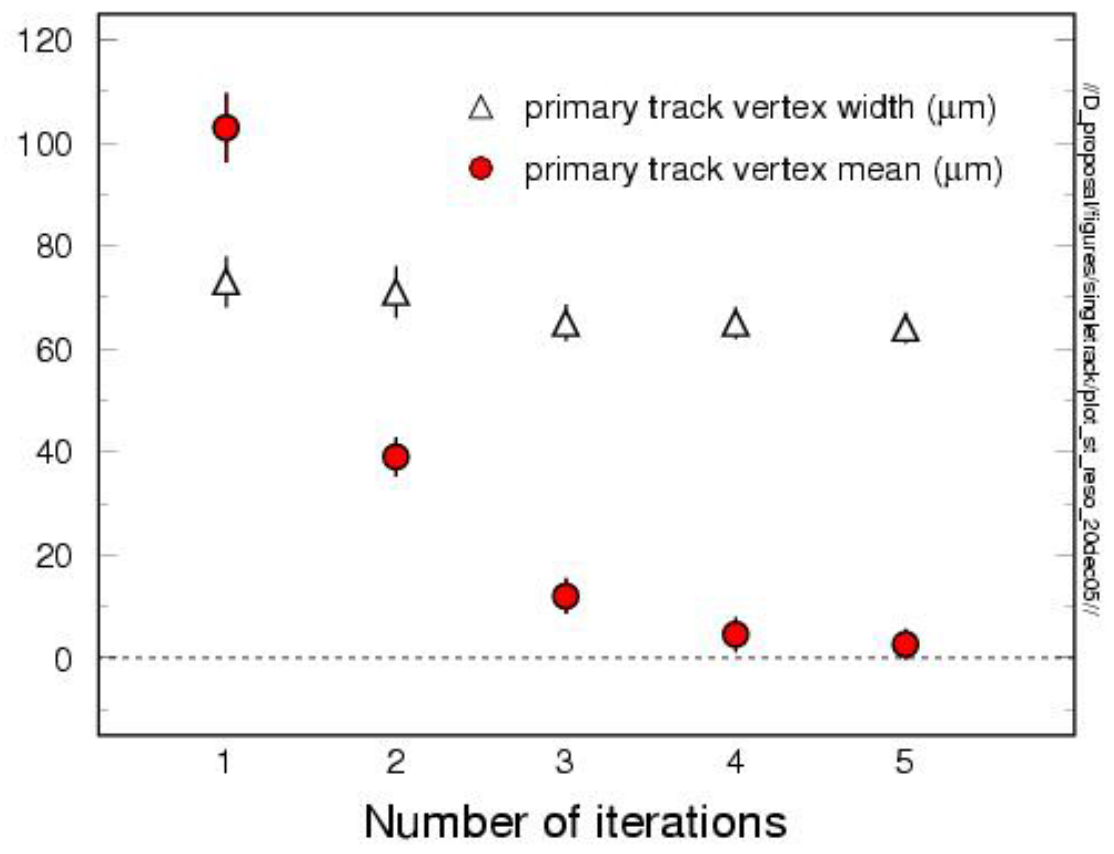

Figure 18: Mean and sigma of the geometric - signed DCA distribution as a function of the number of iterations used to correct the vertex. With each iteration, the previously defined vertex is corrected with the mean of the distribution of the track dca's. The first iteration uses the TPC+SSD defined vertex as a vertex seed. 


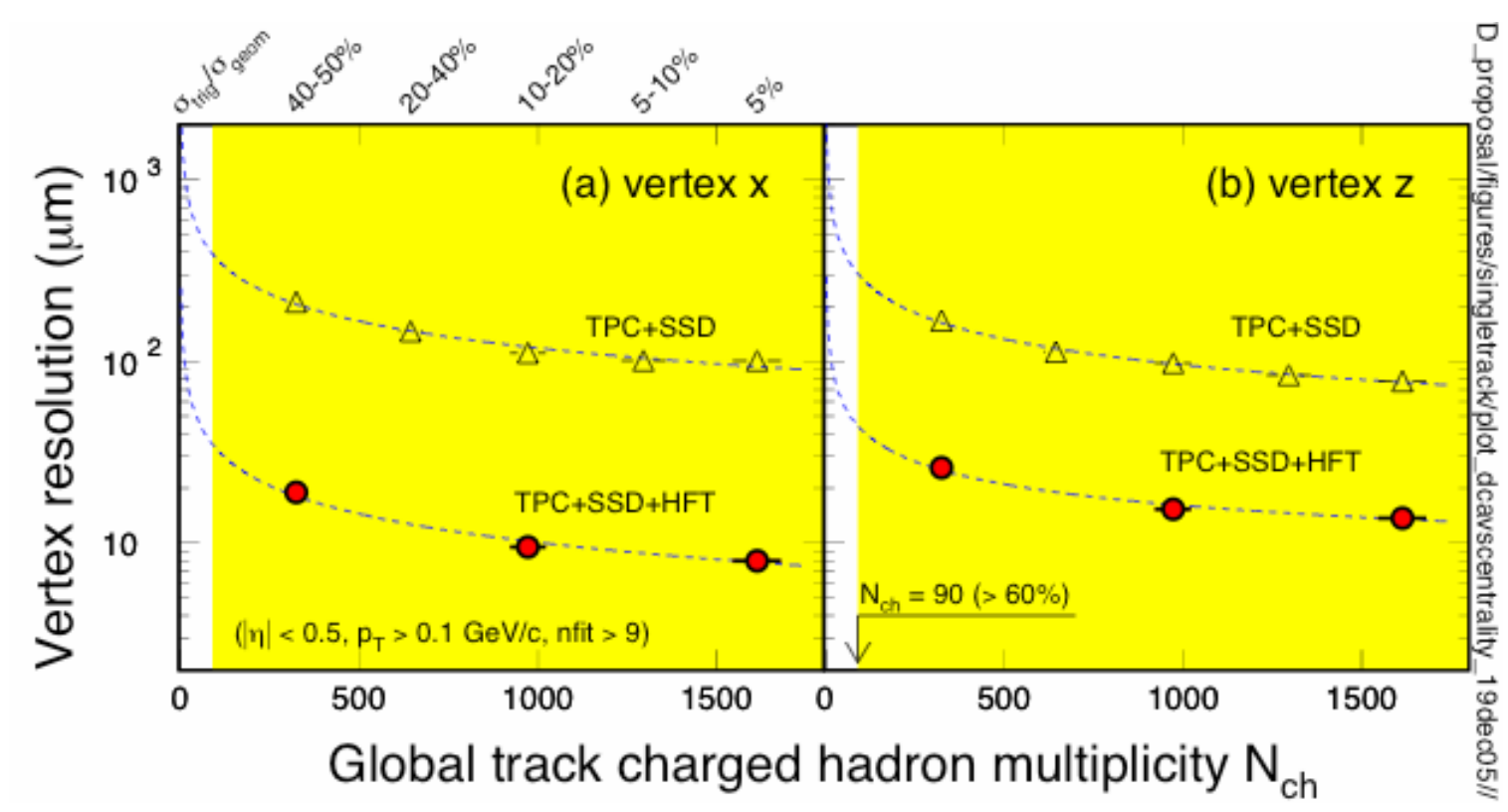

Figure 19: Vertex resolution $\sigma(\mathrm{MC}$ Vertex Position - Reconstructed Vertex position) as a function of collision centrality. The HFT refit vertex shows an order of magnitude improvement over the previously reconstructed primary vertex. a) Vertex resolution in $X$ vs. $N_{c h}$. b) Vertex resolution along the beam direction. Problems in the vertex fit in the $z$ direction drive the distribution wider than in the transverse direction. The highlighted area demarcates the centrality region with minimum (or better) acceptable TPC+SSD vertex resolution. This corresponds to $0 \%-60 \%$ most central events.

Application of a vertex constraint before adding detector hits is a new tool for the STAR software chain, and this technique requires minor modifications of the STAR reconstruction code. The performance of this algorithm has been studied in terms of the efficiency and the expected rate of false hit associations.

The efficiency of the reconstruction package to find and correctly associate HFT hits is defined as:

$$
\varepsilon=\frac{N_{\text {reconstructred }}}{N_{M C, \text { Accepted }}}
$$

where the reconstructed tracks are matched to their corresponding Monte Carlo track at the hit level. Tracks that do not match, or which only partially match, to a Monte Carlo track, do not contribute to the efficiency. Hit matching requirements are set to $33 \%$ in the TPC (15 hits), 100\% in the SSD (1 hit) and 100\% in the HFT (2 hits). Tracks with one or more non-matched HFT hit are designated as 'ghost' tracks. The measured efficiency as a function of $\mathrm{p}_{\mathrm{T}}$ is $\sim 50 \%$ for $\mathrm{p}_{\mathrm{T}}>1 \mathrm{GeV} / \mathrm{c}$ (see Figure 20) and the level of ghosting is $14 \%$ over the same range. 


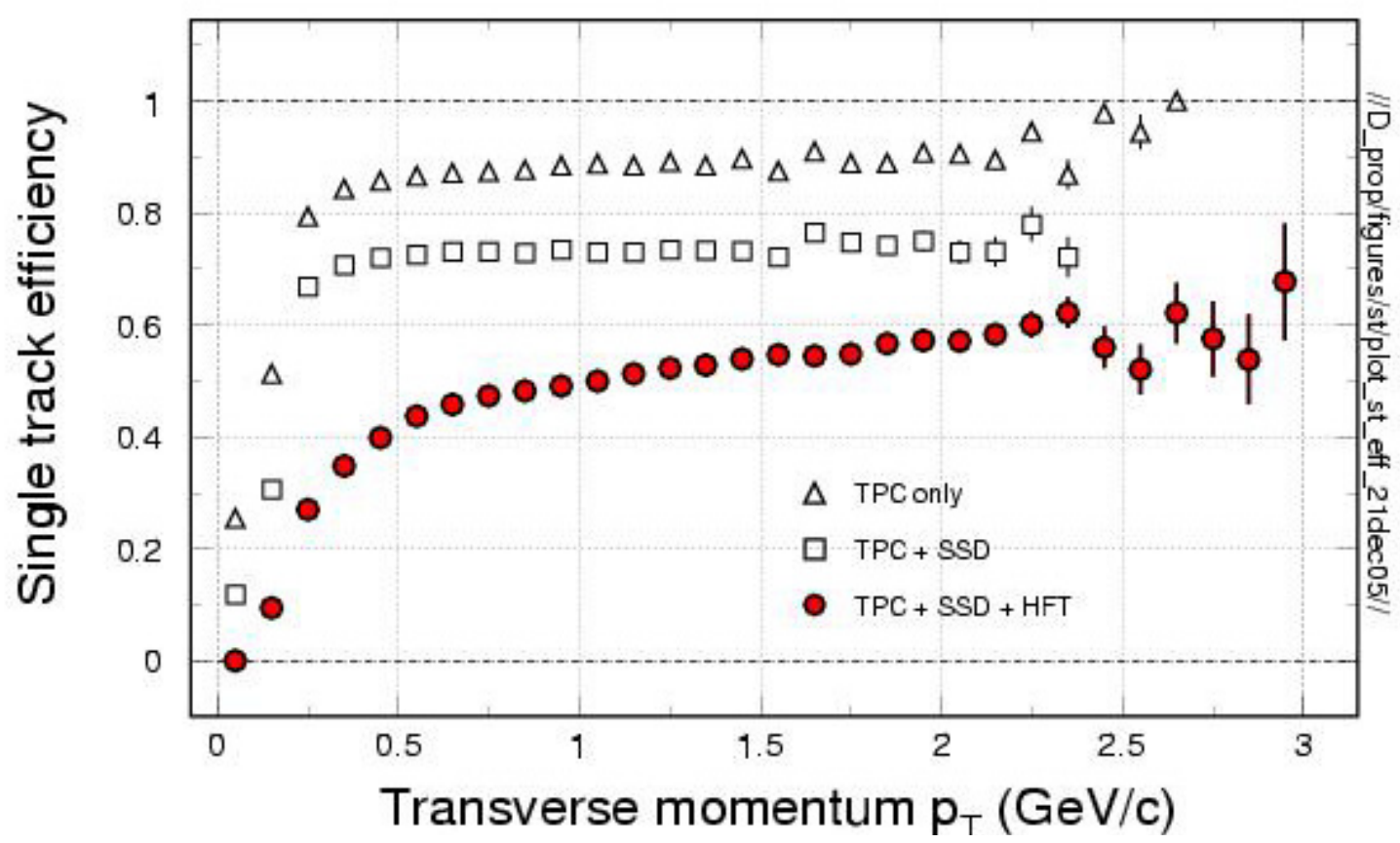

Figure 20: The efficiency for finding tracks in central Au + Au collisions in the STAR TPC, SSD, and the HFT. Accepted tracks have 15 TPC hits (or more), 1 SSD hit, and 2 HFT hits that match to a single Monte Carlo track. The quoted efficiency is for pions in the detectors acceptance; $|\eta|<0.5$

\subsubsection{Primary Track Reconstruction Performance}

The performance of the HFT was simulated using tracks from central $\mathrm{Au}+\mathrm{Au}$ collisions at ${\sqrt{\mathrm{S}_{\mathrm{NN}}}}=200 \mathrm{GeV}$ which were generated by the "MEVSIM" code and simulated using single particle tracks. The simulated tracks and events were processed through GEANT and, finally, a detailed response function for the Time Projection chamber (TPC) and a response function for the Silicon Drift Detector (SSD) were applied to the data. These response functions take into account physical effects in the detector and the subsequent electronics. For the HFT, we smeared the Monte Carlo hits by a Gaussian function with a width of $\sigma=6 \mu \mathrm{m}$ in the $\mathrm{x}-\mathrm{y}$-plane and in the z-direction. The $4 \mathrm{~ms}$ read-out speed of the $1^{\text {st }}$ generation HFT will lead to pile-up of $120 \mathrm{Au}+\mathrm{Au}$ collisions in the HFT at four times the RHIC design luminosity. We account for this by randomly uploading hits on top of the Monte Carlo generated hits in the HFT. The topic of pile-up is discussed in detail in section 3.7

Tracks with at least 15 hits in the TPC, 1 hit in the SSD and 2 hits in the HFT detector were reconstructed with the STAR inner-tracker code. Figure 20 shows the efficiency for tracking pions with $|y|<0.5$ in various detector configurations. The TPC results are designated by the open triangles, TPC + SSD by squares, and the TPC + SSD + HFT detector by filled dots (red). Note that the TPC tracking efficiency limits the efficiency of the SSD at low transverse momentum and, in addition, the efficiency of the HFT at 
low momentum is affected by ghost tracks and event pileup. But at high momentum, the acceptance of the detectors is quite uniform. Of the TPC and SSD tracks above 700 $\mathrm{MeV} / \mathrm{c}$ traced to the HFT detector volume, about $70 \%$ have correctly matched HFT hits. The absolute efficiency for STAR tracking with the HFT (using the TPC, SSD and HFT) is $50 \%$ or above at a transverse momentum larger than $700 \mathrm{MeV} / \mathrm{c}$. Simulations using mono-energetic pions show that the efficiency stays approximately constant up to 20 $\mathrm{GeV} / \mathrm{c}$.

In the high multiplicity environment of a central $\mathrm{Au}+\mathrm{Au}$ collision, there can be ambiguities in finding the next hit on a track while following a track along its path. A ghost track is defined to be a reconstructed track with at least one hit from a different Monte Carlo track. With the present tracking algorithm, the number of ghost tracks in the TPC is less than $1 \%$ compared to all reconstructed tracks. The HFT is more restrictive, however, because it is continuously read out; events will pile up between successive reads of the Si chip, which occur every $4 \mathrm{~ms}$.

Figure 21 shows the number of ghost tracks observed in the full HFT tracking system. At low momentum, ghost tracks dominate due to multiple Coulomb scattering. The bulk of the ghost tracks appear below $0.5 \mathrm{GeV} / c$ and the effect of these miss-identified tracks can be seen in Figure 20 because the tracking efficiency for the HFT drops steeply below $0.5 \mathrm{GeV} / c$. The ratio of ghost tracks to good tracks is $20 \%$ at $0.5 \mathrm{GeV} / c$ and saturates at $14 \%$ above $0.7 \mathrm{GeV} / c$ (see the right hand panel of Figure 21 ).
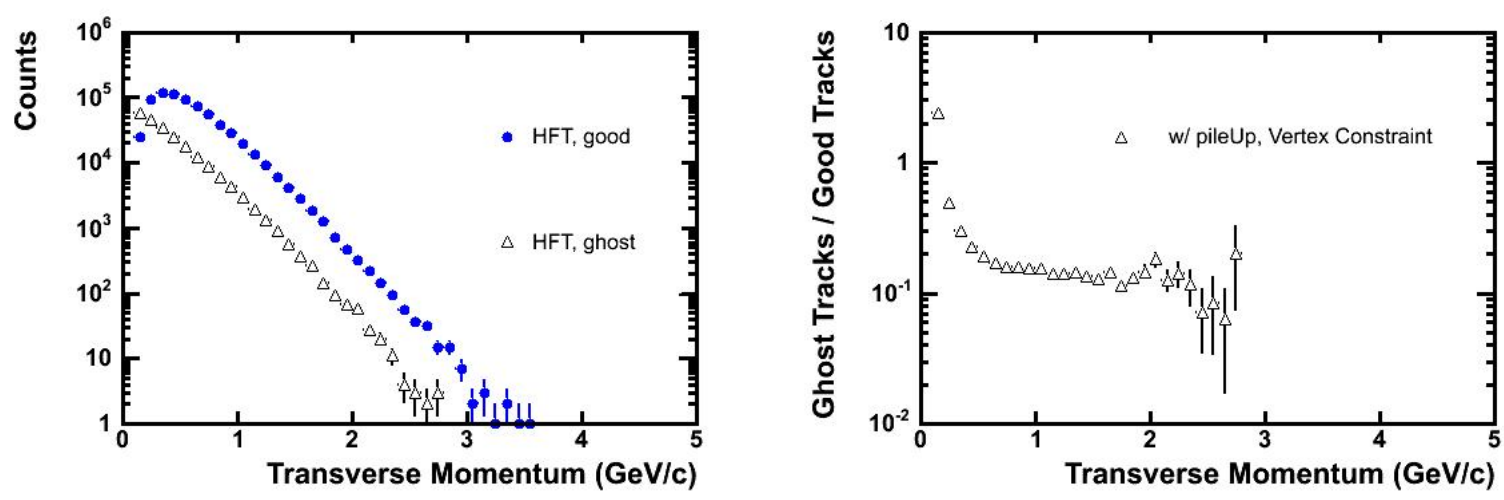

Figure 21: Reconstructed tracks with false HFT hits. Left - The number of ghost tracks and reconstructed tracks passing through the pixels of the HFT as a function of $\mathrm{p}_{\mathrm{T}}$. Right - The ratio of ghost tracks to good tracks. The increased ghosting rate at lower transverse momentum is a direct consequence of multiple Coulomb scattering, and is the motivating factor to make the detector as thin as possible. 


\subsubsection{Analytic Resolution Calculations and Expected Performance}

To verify our detailed STAR software simulation, we have made several cross checks. One method is to do a simple analytic calculation of efficiency and ghosting versus the transverse momentum.

The probability to pick up the correct hit can then be described by the following equation:

$$
\int_{0}^{a} \frac{r}{\sigma^{2}} e^{-r^{2}\left(\pi \rho+\frac{1}{2 \sigma^{2}}\right)} d r
$$

The probability to pick up the wrong hit is described by the equation:

$$
\int_{0}^{a} \frac{r}{\sigma^{2}} e^{-\frac{r^{2}}{2 \sigma^{2}}}\left(1-e^{-\pi r^{2} \rho}\right) d r
$$

where $\sigma$ is the uncertainty in the track extrapolation from the outer detectors to the target detector, $\rho$ is the background hits density, and ' $a$ ' (the upper limit of the integral) is the search cone radius. The analytic calculation of the quantity $\sigma$, includes contributions from multiple Coulomb scattering ${ }^{85}$, the detector resolution, and the uncertainty in the position of the track due to the quality of the fit given by the tracking software. ${ }^{86}$. The quantity $\rho$ includes contributions from the pile-up, other tracks in the current event, and ultraperipheral collision (UPC) electrons.

We discovered that track fitting errors in the standard STAR software are the dominant term in the calculation of $\sigma$ while the detector resolution drops out due to the extremely high resolution of the detectors. So in the discussion presented here, we have set $\sigma$ equal to the search radius used by the tracking software at each detector layer. This suggests that the tracking software can be improved and future simulations may someday be better than what we present here.

Figure 23 shows the efficiency and ghost rate results using these equations. The vertex, SSD, HFT, and TPC search radii are assumed to be $300 \mathrm{um}, 60 \mathrm{um}, 90 \mathrm{um}$, and $1.5 \mathrm{~mm}$, respectively. There is excellent agreement in sub-panels (a), (b), (c) and (d) between efficiency and ghost rate; the analytic calculations match the characteristics and shape of the distributions found with the full Monte Carlo simulations. The larger the efficiency, the smaller the ghost rate. The analytic calculations can reproduce the shape of the distribution as a function of $\mathrm{p}_{\mathrm{T}}$. At high $\mathrm{p}_{\mathrm{T}}$, the efficiency is limited by the detector resolution. The efficiency decreases quickly below $1 \mathrm{GeV} / \mathrm{c}$ due to multiple Coulomb scattering, which causes a large rise in the track projection errors at low $\mathrm{p}_{\mathrm{T}}$. The efficiency decreases consistently with an increase of the luminosity. 

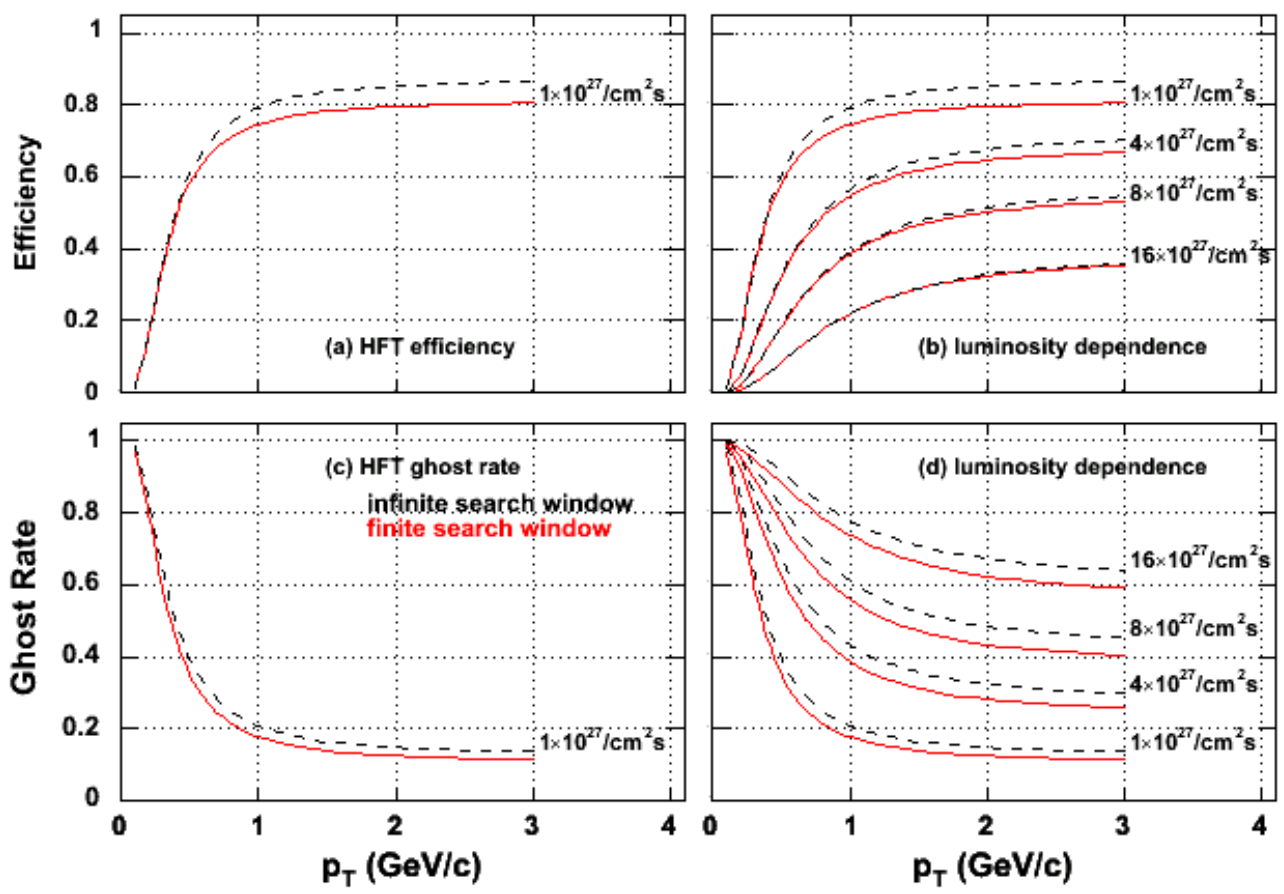

Figure 22: Efficiency (a) and ghost rate (b) of the HFT, using an analytic calculations. The luminosity dependence is given in (c) and (d) for 1-4 times the current peak luminosity. Finite and infinite search windows are represented by solid- and dashed- line, respectively. The efficiency and ghost rate for each layer of the HFT are calculated separately and combined into that for full HFT. The vertex, SSD, HFT, and TPC search radii are assumed to be $300 \mathrm{um}, 60 \mathrm{um}, 90 \mathrm{um}$, and $1.5 \mathrm{~mm}$, respectively. At nominal RHIC luminosity $1 \times 10^{27} / \mathrm{cm}^{2}$ s, the density of background hits $\left(\mathrm{hits}^{2} / \mathrm{cm}^{2}\right)$ are summarized in Table 10. It will be necessary to upgrade the sensors for the full RHICII luminosities.

\subsection{Open Charm Reconstruction Simulation}

As previously discussed, particles containing charm and beauty quarks are the probes relevant for the main thrust of our physics objectives. Charm and beauty quarks occur in a wide variety of hadrons, and these hadrons decay into a large number of different channels. To demonstrate the power of the HFT, we have simulated several specific charm meson decay channels, including $\mathrm{D}^{0} \rightarrow \mathrm{K}^{-} \pi^{+}$and $\mathrm{D}_{\mathrm{s}}^{+} \rightarrow \mathrm{K}^{-} \pi^{+} \mathrm{K}^{+}$, because of their large relative branching ratios. Table 5 displays some of the properties of these channels. 


\begin{tabular}{|c|c|c|c|}
\hline particle & Daughters & $\mathbf{C}_{\tau}(\mu \mathrm{m})$ & Mass (GeV) \\
\hline $\mathrm{D}^{0}$ & $\mathrm{~K}^{-} \pi^{+}(3.8 \%)$ & 123 & 1.8646 \\
\hline$D^{ \pm}$ & $\mathrm{K}^{-} \pi^{+} \pi^{+}(9.2 \%)$ & 312 & 1.8694 \\
\hline$D_{s}$ & $\begin{array}{l}\mathrm{K}^{+} \mathrm{K}^{-} \pi^{+}(4.4 \%) \\
\pi^{+} \pi^{+} \pi^{-}(1.0 \%)\end{array}$ & 147 & 1.9683 \\
\hline$\Lambda_{c}$ & $\mathrm{p} \mathrm{K}^{-} \pi^{+}(5.0 \%)$ & 59.9 & 2.2849 \\
\hline
\end{tabular}

Table 5: Open charm hadron properties

Signal and background events are generated separately. The signal consists of one $\mathrm{D}^{0}$ or $\mathrm{D}_{\mathrm{s}}{ }^{+}$per event. The transverse momentum distribution of the charmed hadrons follows a Boltzman distribution which reproduces the $\left\langle p_{T}>\right.$ of D-mesons as measured by STAR in $\mathrm{d}+\mathrm{Au}$ collisions at ${\sqrt{\mathrm{S}_{\mathrm{NN}}}}=200 \mathrm{GeV}$ and the rapidity distribution suggested by perturbative QCD calculations applying the program code Pythia ${ }^{18}$. The background is simulated using the MevSim event generator parameterized to reproduce the experimentally measured particle multiplicities in $\mathrm{Au}+\mathrm{Au}$ collisions at ${\sqrt{\mathrm{S}_{\mathrm{NN}}}}=200 \mathrm{GeV}$. Our parameterization is accurate for particles below $3 \mathrm{GeV} / \mathrm{c}$. It may underestimate the background above this momentum. The distributions of reconstructed D-meson signal and background were scaled to match the expected D-meson production per central $\mathrm{Au}+$ $\mathrm{Au}$ collision ${ }^{43}$. Also, the higher track reconstruction efficiency in single $\left(\mathrm{D}^{0}\right.$ signal) events compared to central $\mathrm{Au}+\mathrm{Au}$ collisions (background) was taken into account.

Analysis of the reconstructed tracks to identify the 'signal' particle is done separately for the signal and background events. We use a direct reconstruction approach, where the daughter particles are identified as originating from a vertex displaced from the primary vertex. The invariant mass of the parent particle is then calculated from the kinematics of the daughters. This technique is superior to techniques depending on calculating all track combinations in an event both in signal to noise and analysis speed. The distributions from the analysis of signal and background events are then combined for the finished invariant mass distributions.

The heavy flavor tracker is designed to allow us to directly reconstruct mesons containing charm quarks. If this is true, we should see differences between the charm meson daughter tracks and the background and the primary tracks in several important variables. The distance of closest approach between the tracks and the vertex is an important example of this; see Figure 23. The distributions of reconstructed $\mathrm{D}^{0}$ and $\mathrm{D}^{+}$daughters is compared to the primary track background. The charm meson tracks clearly have a broader distribution, driven by the decay length of the charm mesons. Cutting on the track DCA, then, will improve the signal to noise in the analyses. 


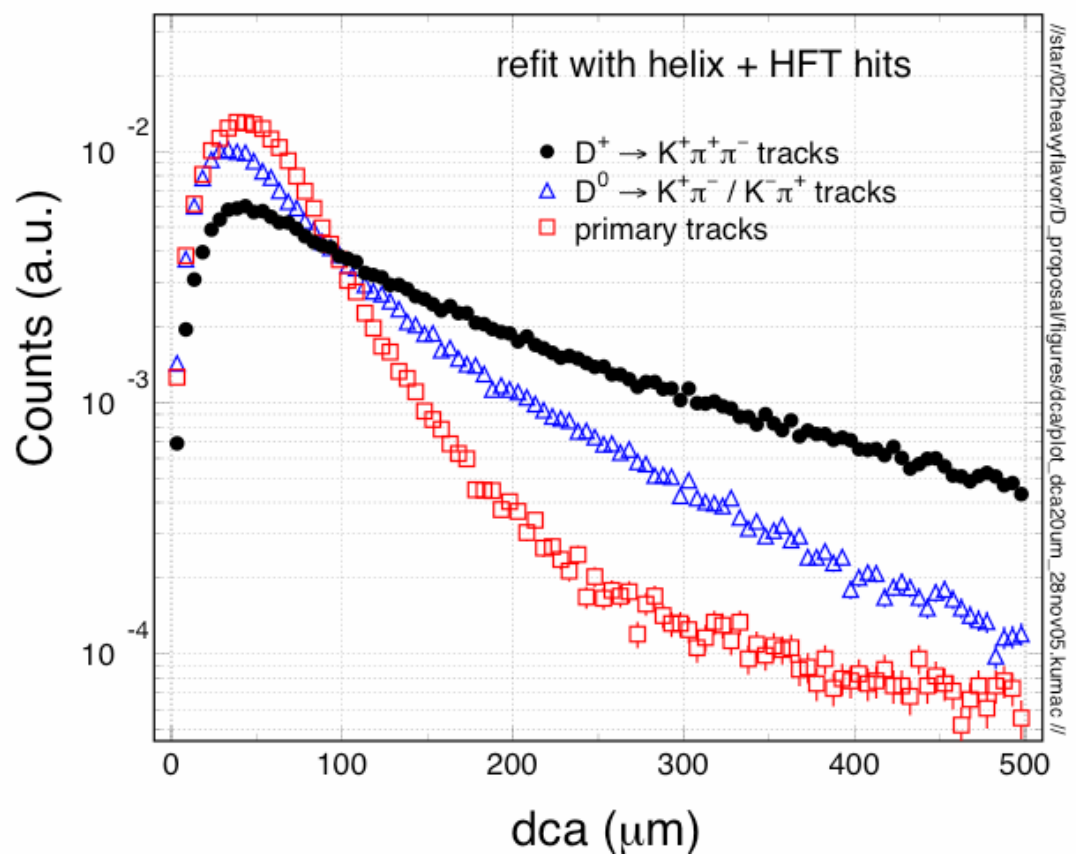

Figure 23: The distance of closest approach (dca) distributions for $\mathrm{D}^{0}$ (triangles) and $\mathrm{D}^{+}$(circles). The results of the dca distribution for primary tracks are shown in squares.

\subsection{1 $\quad D^{0}$ reconstruction}

The HFT detector allows us to identify a $\mathrm{D}^{0}$ decay-vertex by reconstructing the trajectory of its two daughters. Figure 24 shows the topology of the decay.

The following selection criteria were used to separate the $\mathrm{D}^{0}$ signal from background:

- The decay length $l$; with $l$ the distance between the primary vertex and the Dmeson vertex

- The difference, $\Delta m$, between the reconstructed invariant mass and the $\mathrm{D}^{0}$ rest mass

- The distance of closest approach $\mathrm{DCA}_{\pi \mathrm{K}}$, between the two daughter tracks

- Isolation cuts on $\cos (\theta)$, with $\theta$. being the angle between the $\mathrm{D}^{0}$ momentum (vector sum of the two daughter momenta) and the vector joining the primary vertex to the D-meson decay vertex

- Isolation cuts on $\cos \left(\theta^{*}\right)$, with $\theta^{*}$ being the angle between the kaon in the Dmeson center of mass frame and the D-meson momentum 


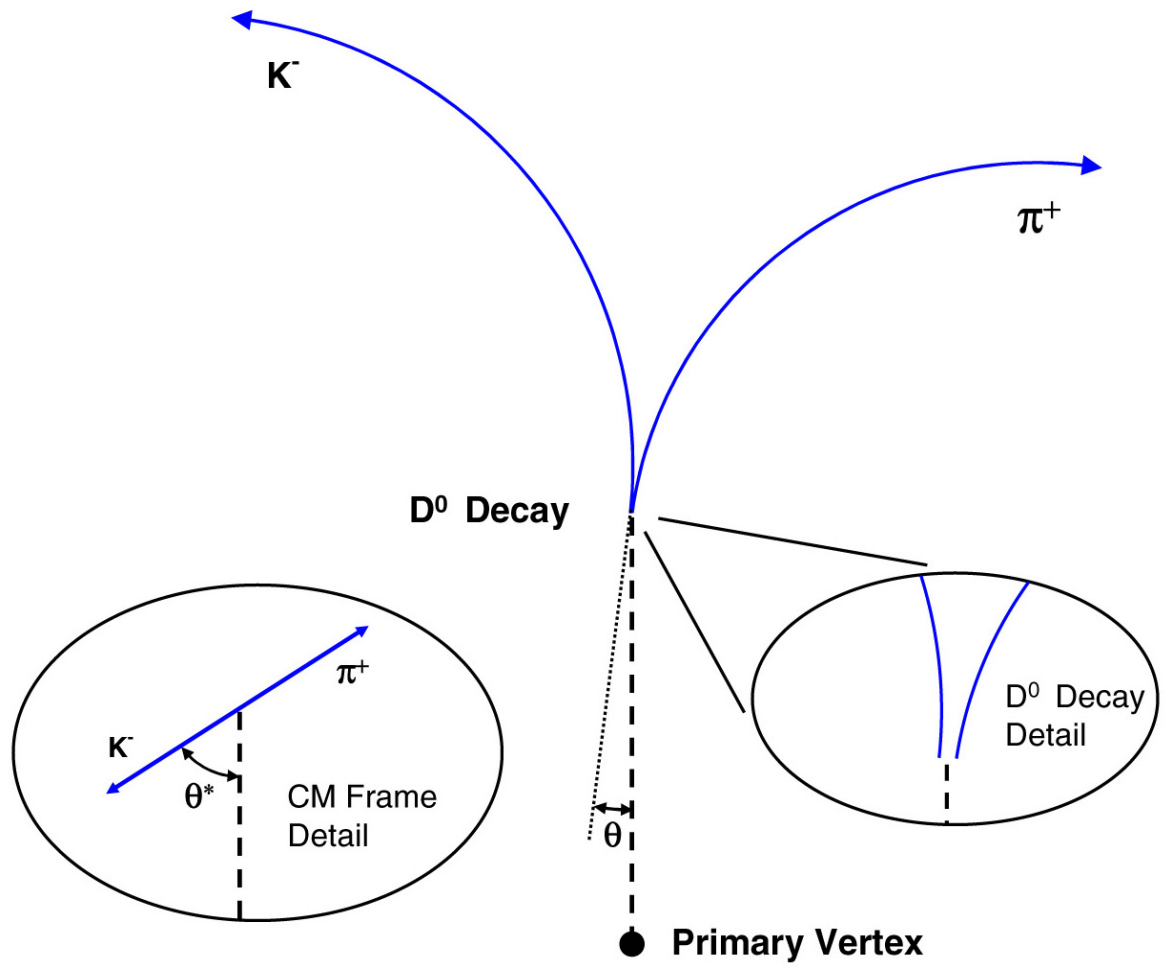

Figure 24: The decay topology for a $\mathrm{D}^{0}$ decaying to a kaon and a pion. Isolation cuts to identify the $D^{0}$ from the background tracks are described in the text.

Charged decay daughters were identified by their specific energy loss in the TPC gas and the time of flight measured in the TOF. For example, to identify pions, we used a $3 \sigma$ cut around the expected band for pions. The width of this distribution is momentum dependent, and was extracted from experimental data. With this method, pions can be separated from kaons up to $700 \mathrm{MeV} / \mathrm{c}$. The TOF detector extends this range up to 1.6 $\mathrm{GeV} / \mathrm{c}$, as demonstrated from existing STAR data with a small version of the full TOF detector. Tracks with a momentum above the range of these PID methods can still be used, but they must be entered into the reconstruction algorithm twice, once under a pion mass assumption, once assuming a kaon mass.

The selection of proper cuts is very important in order to reduce the background. The applied cuts for $\mathrm{D}^{0}$ reconstruction were determined by using the program MINUIT. To achieve the best sensitivity, we selected the cuts to optimize the signal $\left(\mathrm{D}^{0}\right.$ yield, $\left.\mathrm{S}\right)$ to noise $(\mathrm{N})$ ratio $=\mathrm{S} / \sqrt{ }(\mathrm{S}+\mathrm{N})$. See Table 6 for the values that were determined this way. Comparisons of signal and background distributions for various cut variables is shown in Figure 25 (and Figure 28 for the $\mathrm{D}^{+}$), along with the final cut values. Figure 26 demonstrates that the significance, of course, depends critically on the proper choice of cuts. 


\begin{tabular}{|l|c|c|}
\hline Cuts & $\mathbf{D}^{\mathbf{0}}$ & $\mathbf{D}^{+}$ \\
\hline $\mathrm{nFit}>$ & 15 & 15 \\
\hline$|\mathrm{n}|<$ & 1.0 & 1.0 \\
\hline HFT hits $=$ & 2 & 2 \\
\hline dca(global $) \geq$ & & $100 \mu \mathrm{m}$ \\
\hline dca $\left(\mathrm{V}_{0}\right) \leq$ & $35 \mu \mathrm{m}$ & $100 \mu \mathrm{m}$ \\
\hline decay length $\geq$ & $150 \mu \mathrm{m}$ & $150 \mu \mathrm{m}$ \\
\hline $\cos (\vartheta)>$ & 0.996 & 0.85 \\
\hline$\Delta \mathrm{m} \leq$ & $40 \mathrm{MeV}$ & \\
\hline
\end{tabular}

Table 6: The cuts for the $\mathrm{D} 0$ and $\mathrm{D}+$ reconstruction and efficiency analysis. The input $\mathrm{DO}$ and $\mathrm{D}+$ spectra followed an exponential distribution, $<\mathrm{pt}>=1.3 \mathrm{GeV} / \mathrm{c}$.
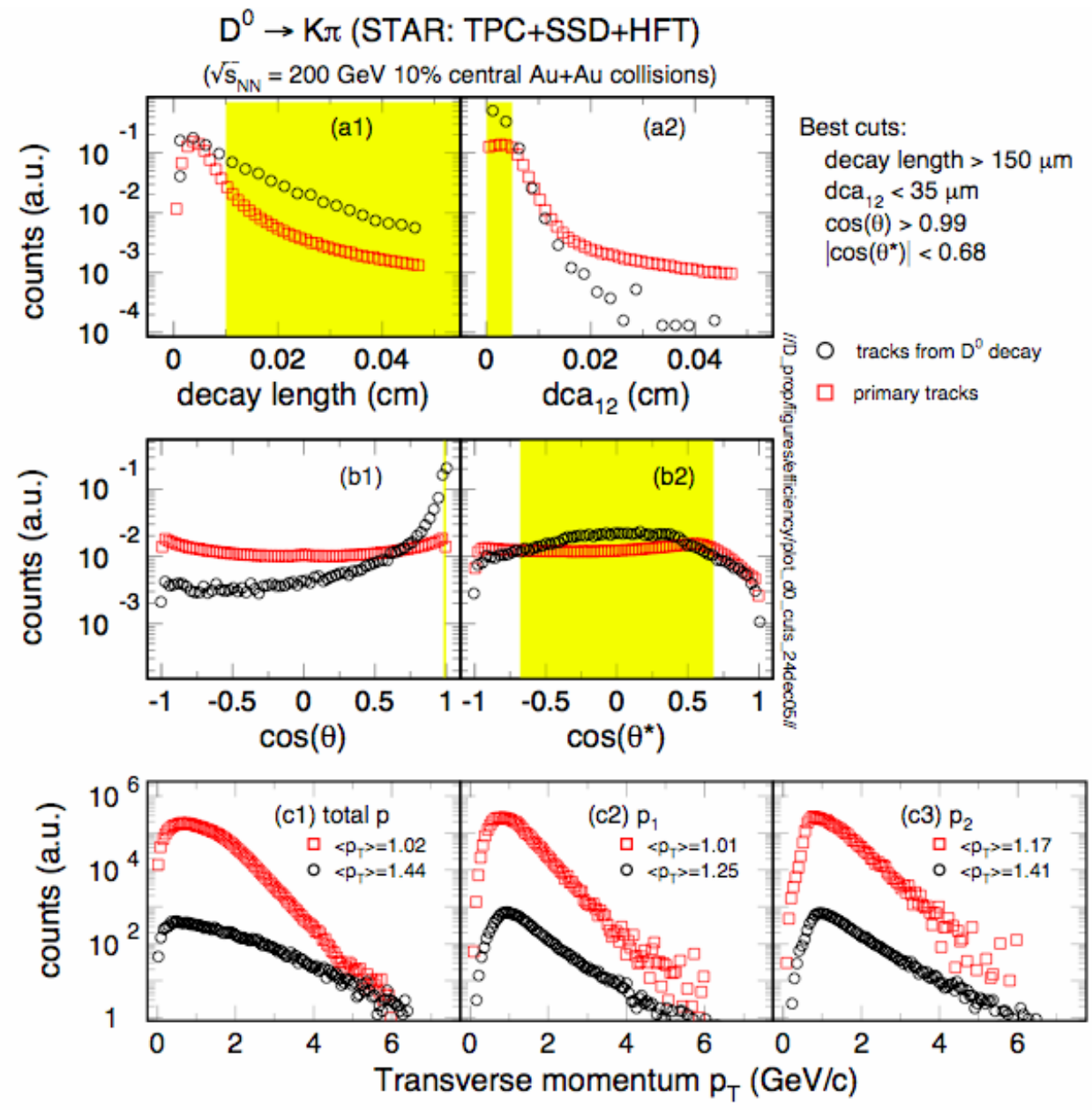

Figure 25: Distribution of quantities used to distinguish signal from background. Panels a1, a2, b1, and b2 compare the difference between background events and D0 mesons for different variables. The yellow shaded area shows the regions where we places cuts to enrich the D0 to background ratio. The lower panels, c1, c2, and c2, show the pt distribution for signal and background event tracks with a $100 \mu \mathrm{m}$ error vertex (c1), $20 \mu \mathrm{m}$ error vertex (c2), and perfect vertex (c3). Central Au + Au collisions are assumed for the background primary tracks. 


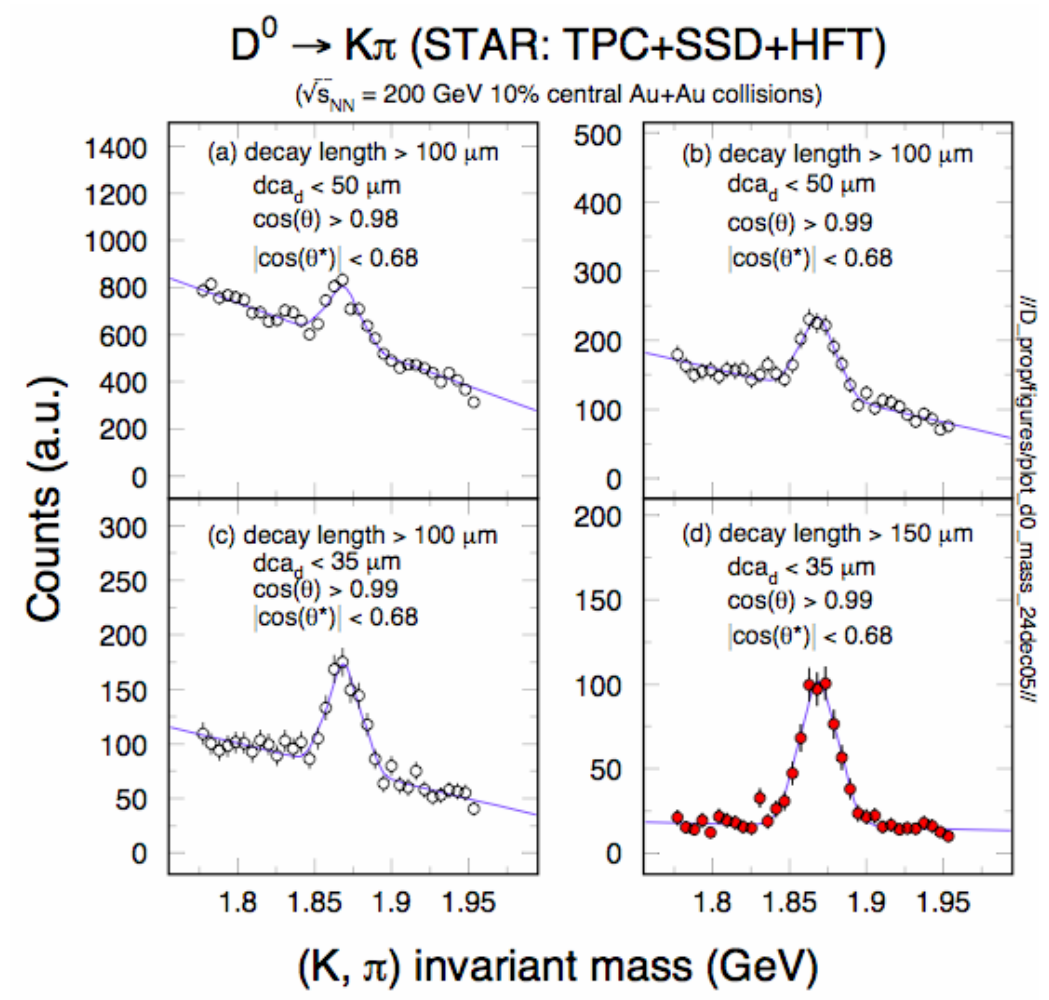

Figure 26: $D^{0}$ invariant mass with different cut sets. The power of the cuts to enhance the signal relative to the background is clear.

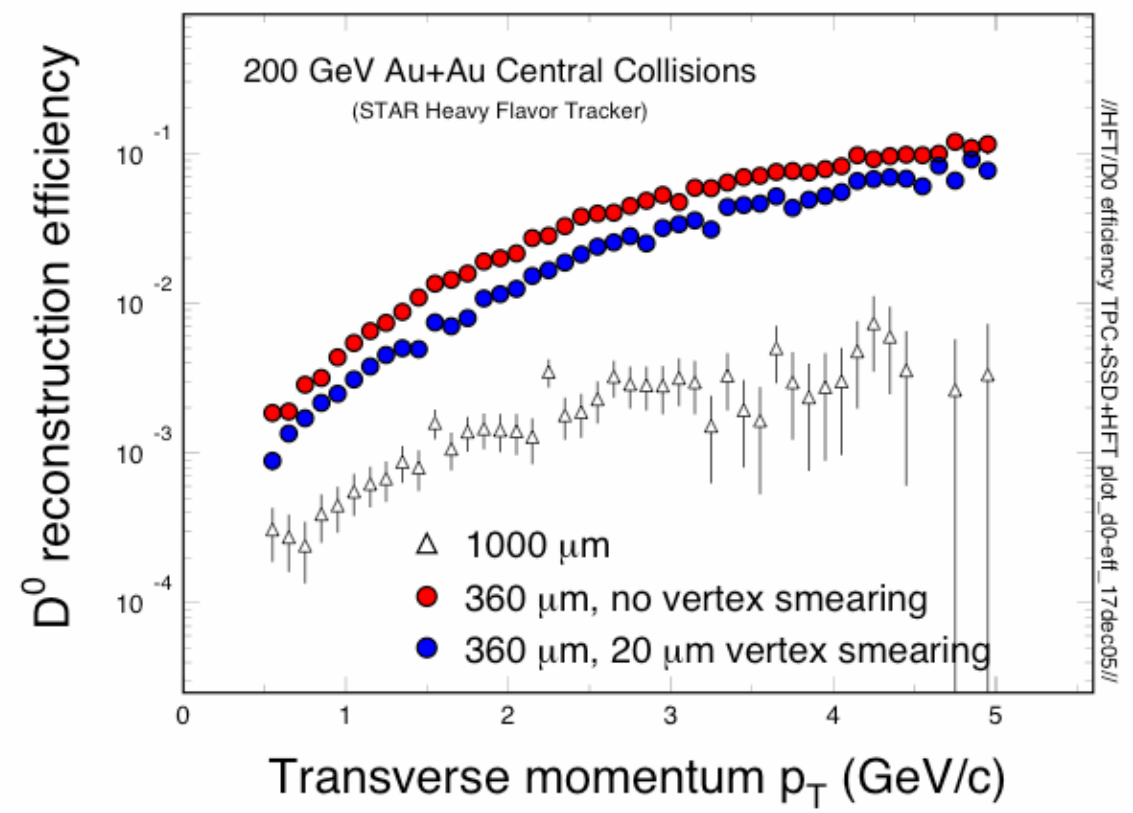

Figure 27: The absolute yield of $\mathrm{D}^{0}$ into the TPC + SSD + HFT divided by the input $\mathrm{D}^{0}$ yield. For comparison, a simulation with thick Si detectors that are similar to the wafers used in the ALICE vertex detector is also shown (1000 $\mu \mathrm{m}$ effective silicon thickness). 
Once we have made these cuts, we can study the reconstruction efficiency. The overall efficiency takes into account acceptance, single track efficiency and $\mathrm{D}^{0}$ reconstruction efficiency. We have studied the $\mathrm{D}^{0}$ detection efficiency for three cases: knowledge of the exact location of the vertex, a vertex that is known to $20 \mu \mathrm{m}$, and an ALICE like detector. Figure 27 shows that the overall reconstruction efficiency increases with increasing momentum and saturates above $3 \mathrm{GeV} / \mathrm{c}$ at a value of about $10 \%$. The efficiency of a $1000 \mu \mathrm{m}$ detector is about an order of magnitude less. ${ }^{*}$

Since the single track efficiency of the HFT is $50 \%$, the maximum $\mathrm{D}^{0}$ reconstruction efficiency can be estimated to be $0.5 \times 0.5$ (tracks) $=25 \%$, not taking into account the $\mathrm{D}^{0}$ acceptance effects. This efficiency directly impacts the feasability of a $\mathrm{D}^{0}$ measurement for topics such as charm flow. Full estimates of the expected rate for detecting $\mathrm{D}^{0}$ s $\mathrm{s}$ and the data needed to accomplish the flow measurement are detailed in section 3.5 In the next section, we will describe a related analysis; the reconstruction of the $\mathrm{D}^{+}$meson.

\subsection{2 $D^{+}$reconstruction}

The dca-distribution, $\cos (\theta)$, and decay-length distribution for both primary tracks (opensquares) and $\mathrm{D}+$ decayed tracks are shown in Figure 28. Clearly the decayed tracks are well separated from the primary track. For $\mathrm{D}^{+}$reconstruction, a slightly different method was used compared to that of the $\mathrm{D}^{0}$. The 'signal event' and 'background event' were mixed together. The distribution of the invariant mass from a $\mathrm{K}$ and two $\pi$ tracks is then formed. The number of tracks used in the background events is consistent with the top $10 \%$ central $\mathrm{Au}+\mathrm{Au}$ collisions at RHIC. The resulting invariant mass distributions for several $\mathrm{p}_{\mathrm{T}}$ bins are shown in Figure 29. For the $\mathrm{p}_{\mathrm{T}}$ bins studied so far, the significance $\mathrm{S} / \sqrt{ }(\mathrm{S}+\mathrm{B})$ is better than 3 .

\footnotetext{
* The results from a silicon sensor with the thickness used in the ALICE detector ( 3 times thicker than the STAR design) results in the $\mathrm{D}^{0}$ reconstruction efficiency dropping by a factor 8 , independent of momentum. To achieve the same statistical significance would then require an 8 times longer data taking period. As a result, we have excluded the ALICE detector technology from our design considerations.
} 


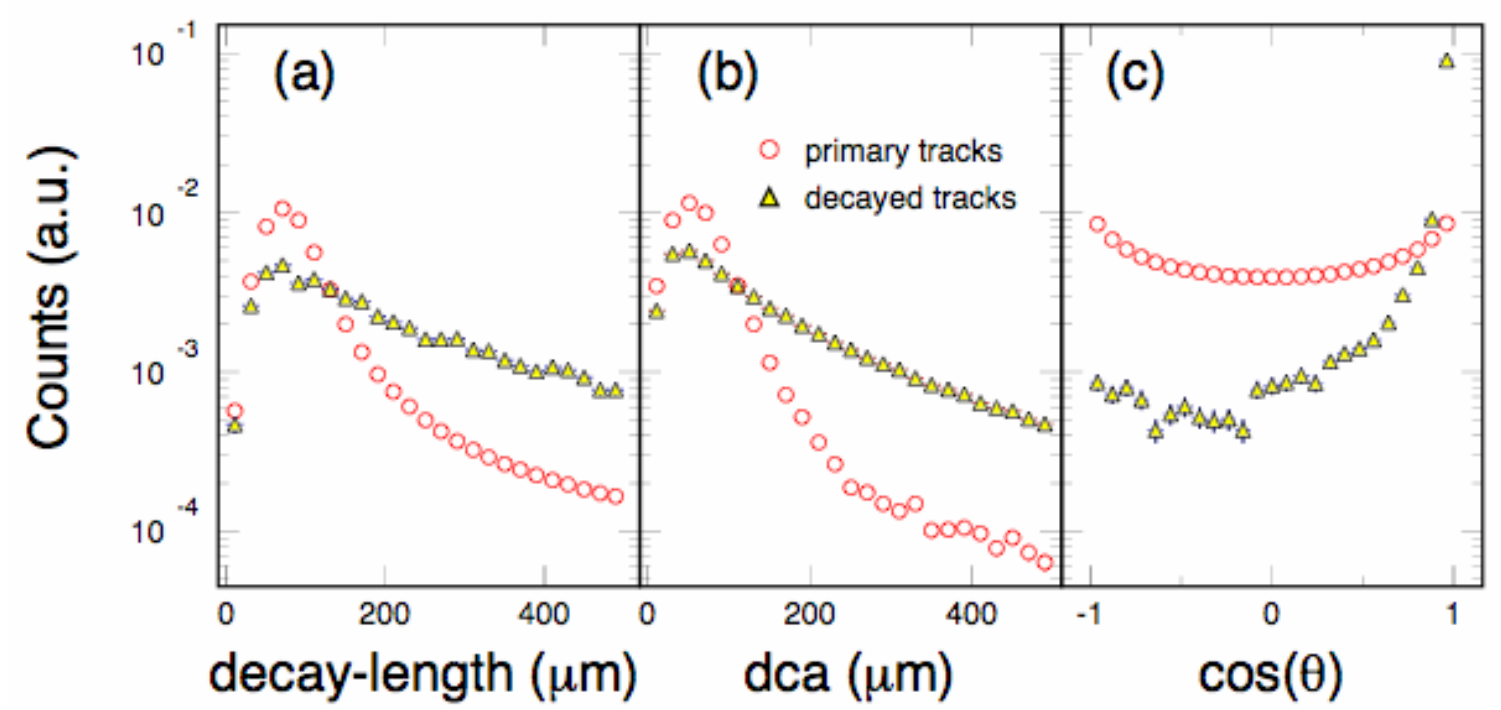

Figure 28: The $\mathrm{D}^{+}$decayed tracks decay-length, dca, and $\cos (\theta)$ distributions (triangles). The same distributions for primary tracks are also shown (squares).

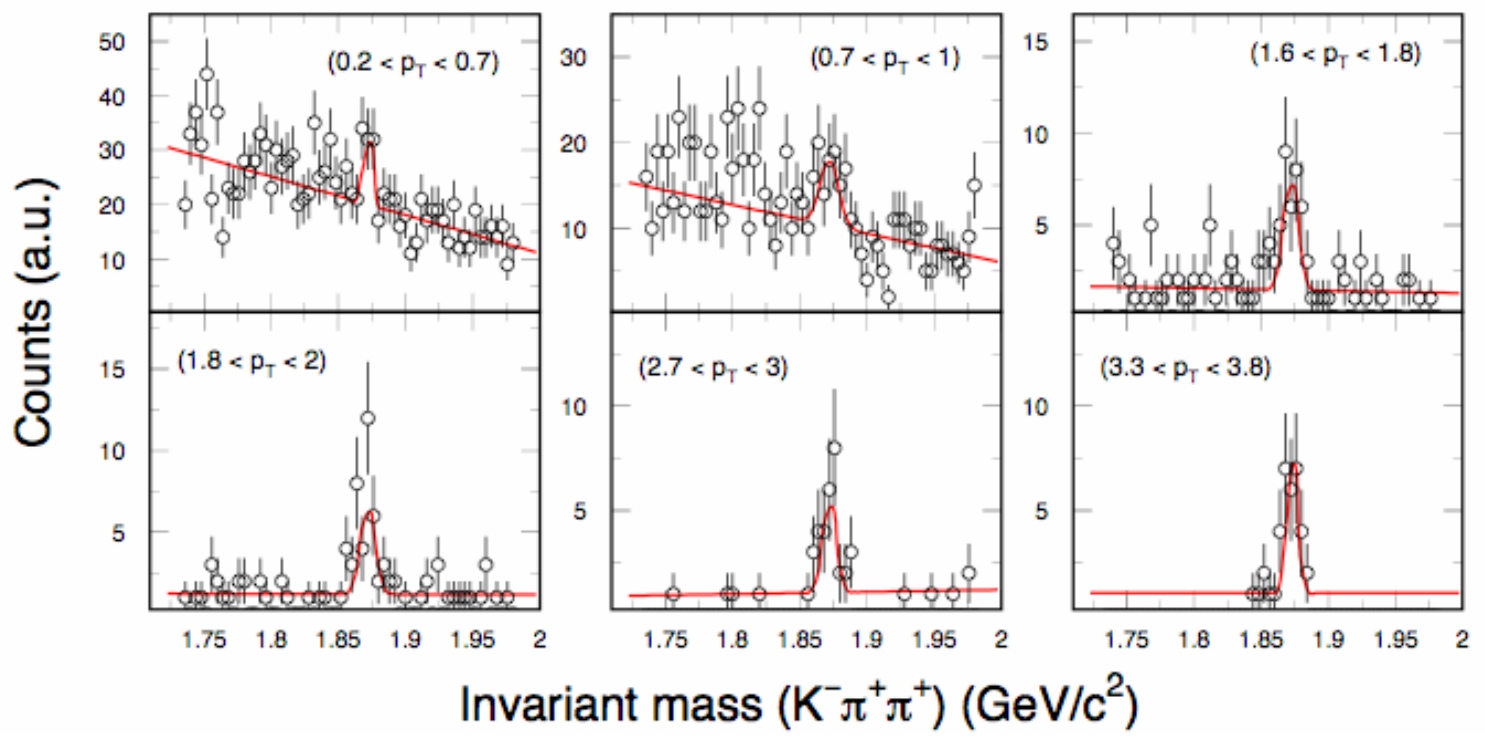

Figure 29: Invariant mass distributions for $\mathrm{D}^{+}$for several $\mathrm{p}_{\mathrm{T}}$ windows. The lines are a polynomial (up to $2^{\text {nd }}$ order) + Gaussian fit.

The final reconstruction efficiencies for $\mathrm{D}^{0}$ and $\mathrm{D}^{+}$mesons are shown in Figure 30. Below $\mathrm{p}_{\mathrm{T}} \sim 0.5 \mathrm{GeV} / \mathrm{c}$ for the Ds, the efficiency is very low since the slow charged daughter particles do not make it into the TPC. Above $\mathrm{p}_{\mathrm{T}} \sim 1 \mathrm{GeV} / \mathrm{c}$, both efficiencies increases as a function of the transverse momentum. The large difference between $\mathrm{D}^{0}$ and $\mathrm{D}^{+}$efficiencies is caused by the topological cuts used for the reconstruction. Although the numbers are not yet fully optimized, these efficiencies are already usable for data analysis. 


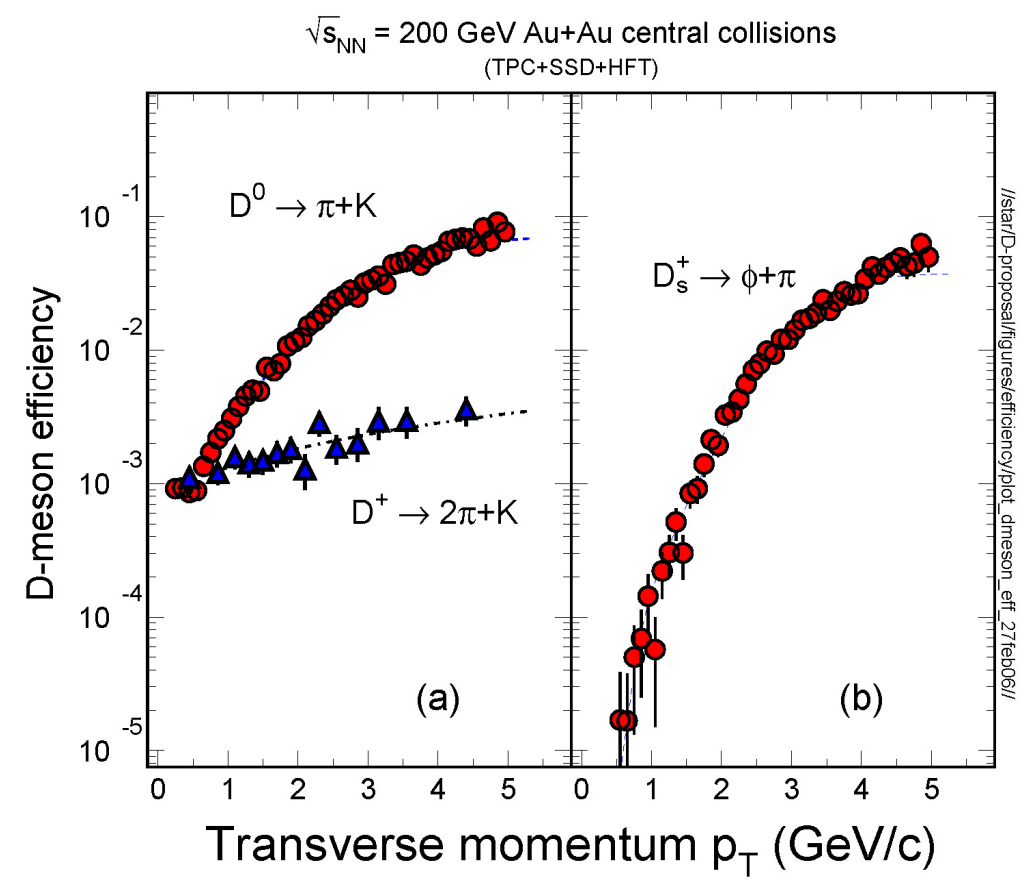

Figure 30: D-meson efficiencies as a function of transverse momentum, together with fits. to the distributions. The $\mathrm{D}^{0}$ efficiency approaches $10 \%$, while the $\mathrm{D}^{+}$efficiency approaches $.3 \%$.

\subsection{Charm Elliptic Flow}

As stated in Section 2, a measurement of charm elliptic flow is one of the main goals of the HFT. The measurement is necessary to understand the degree of thermalization in the partonic phase of a relativistic collision. To study the sensitivity of the HFT for such a measurement, we will focus on the capability of the HFT to measure the flow of $\mathrm{D}^{0}$ mesons. The simulated $\mathrm{D}^{0}$ transverse momentum distribution was generated from 200 $\mathrm{GeV} p+p$ collisions in Pythia ${ }^{46}$. The shape follows a power-law, and the integrated yield is fixed to the yield measured at mid-rapidity at $\mathrm{RHIC}^{17}$, or $\mathrm{dN} / \mathrm{dy}=0.03$. The default value of $\mathrm{dN} / \mathrm{dy}$ in Pythia is about a factor of 3 lower.

For the $10 \%$ most-central and $0-80 \%$ minimum bias $\mathrm{Au}+\mathrm{Au}$ collision events, the number of binary collisions are 950 and 290 , respectively ${ }^{87}$. The resulting distributions together with the efficiencies described previously are shown in Figure 31. The final measured particle yield versus $\mathrm{p}_{\mathrm{T}}$ is also shown. Assuming $10 \%$ statistical errors in each $\mathrm{p}_{\mathrm{T}}$ bin, the number of events required can be easily calculated, and the results are given in Table 7. At $\mathrm{p}_{\mathrm{T}} \sim 10 \mathrm{GeV} / \mathrm{c}$, a good $\mathrm{R}_{\mathrm{AA}}$ measurement requires about 7.5 billion $\mathrm{p}+\mathrm{p}$

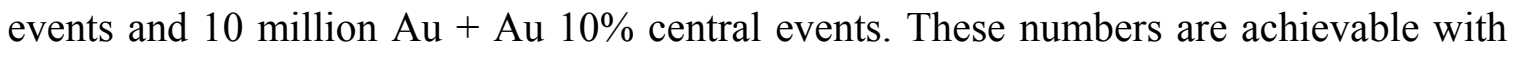
the planned RHIC luminosity ${ }^{88}, 60 \%$ duty factor, and the current STAR detector system in a week of RHIC running. 


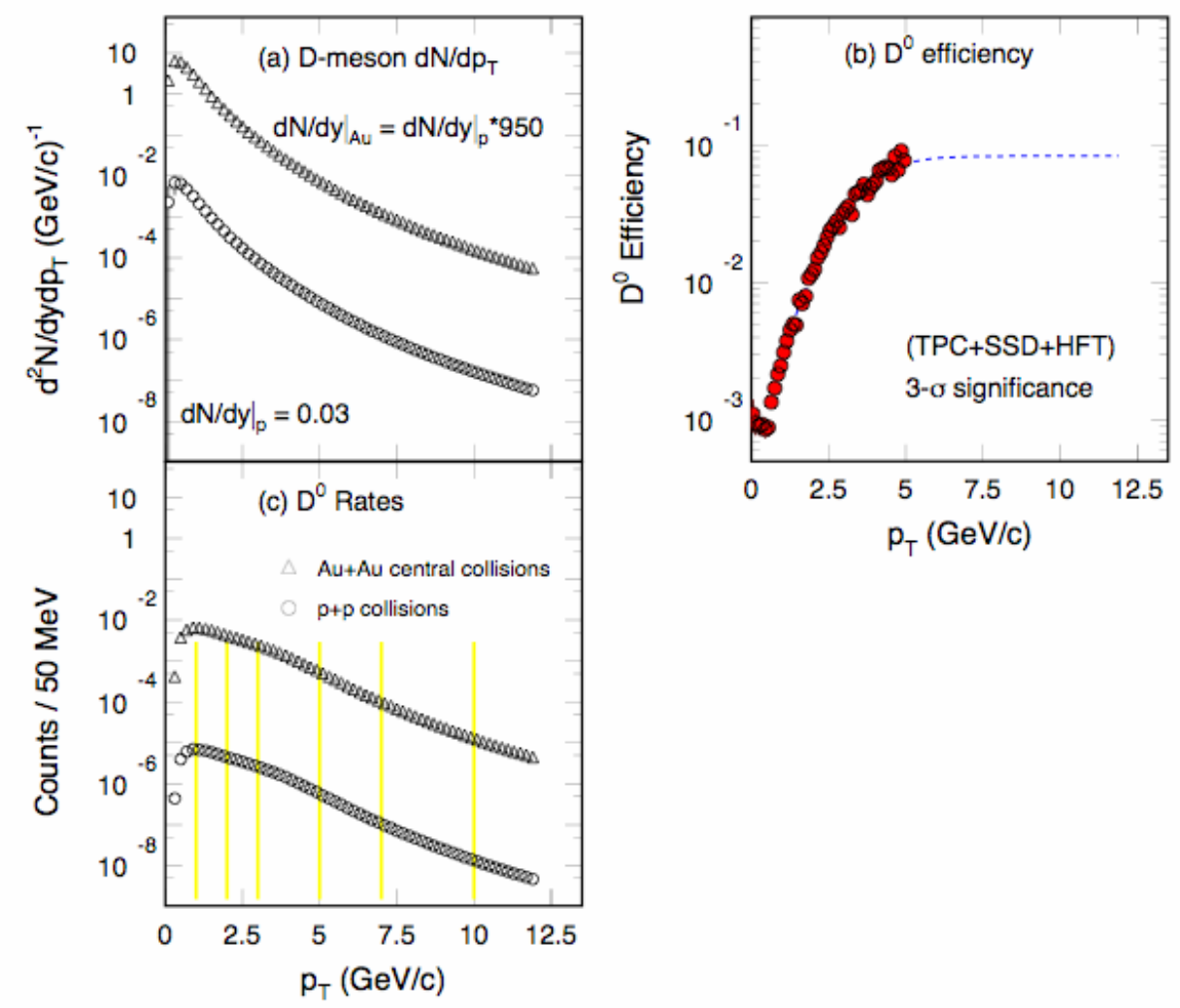

Figure 31: D-meson rates estimates: (a) $d N / d p_{T}$ distributions for $D$-mesons. The integrated yield $\mathrm{dN} / \mathrm{dy}=\mathbf{0 . 0 3}$ as measured in $\mathrm{p}+\mathrm{p}$ collisions at $200 \mathrm{GeV}$ [Ref. 17]. The number of binary collisions $\left(\mathrm{N}_{\text {bin }}\right)=950$, corresponds to the top $10 \%$ most central Au + Au collisions at RHIC and is used to scale the Au + Au collisions; (b) 3- $\sigma$ significance $D^{0}$ efficiency with TPC+SSD+HFT. The dashed-line is the fit to the simulation results; (c) $D^{0}$ meson rates from $p+p$ and top $10 \%$ central Au $+A u$ collisions at $200 \mathrm{GeV}$.

\begin{tabular}{|l|l|l|l|l|}
\hline $\mathbf{p}_{\mathbf{T}}(\mathbf{G e V} / \mathbf{c})$ & $\Delta \mathbf{p}_{\mathbf{T}}(\mathbf{G e V} / \mathbf{c})$ & $\begin{array}{l}\text { \# of Events } \\
(\mathbf{p}+\mathbf{p})\end{array}$ & $\begin{array}{l}\text { \# of Events } \\
\mathbf{0 - 1 0 \%} \mathbf{A u}+\mathbf{A u} \\
\left(\mathrm{N}_{\text {bin }}=950\right)\end{array}$ & $\begin{array}{l}\text { \# of Events } \\
\mathbf{0 - 8 0} \% \mathbf{A u}+\mathbf{A u} \\
\left(\mathrm{N}_{\text {bin }}=290\right)\end{array}$ \\
\hline 1.0 & 0.5 & $44 \times 10^{6}$ & $0.45 \times 10^{6}$ & $1.75 \times 10^{6}$ \\
\hline 2.0 & 0.5 & $70 \times 10^{6}$ & $0.45 \times 10^{6}$ & $1.75 \times 10^{6}$ \\
\hline 3.5 & 1.0 & $70 \times 10^{6}$ & $0.45 \times 10^{6}$ & $1.75 \times 10^{6}$ \\
\hline 5.5 & 1.0 & $350 \times 10^{6}$ & $0.75 \times 10^{6}$ & $3 \times 10^{6}$ \\
\hline 7.5 & 1.0 & $1200 \times 10^{6}$ & $3.5 \times 10^{6}$ & $11 \times 10^{6}$ \\
\hline 10.5 & 1.5 & $7500 \times 10^{6}$ & $9 \times 10^{6}$ & $30 \times 10^{6}$ \\
\hline
\end{tabular}

Table 7: An estimate of the number of events required to achieve $10 \%$ statistical errors for the spectra measurements of $200 \mathrm{GeV} p+p$ collisions and 0-10\%/ 0-80\% Au + Au collisions. A powerlaw shape $p_{\mathrm{T}}$ distribution from the $p+p$ collision has been assumed with the mid-rapidity $\mathrm{dN} / \mathrm{dy}=$ 0.03 [Ref. 17]. The number of binary collisions is from Glauber calculations [Ref. 23]. 
In order to make a rate estimate relevant for the charm $v_{2}$ measurement, we need to make further assumptions about the $\mathrm{p}_{\mathrm{T}}$ dependence of the anisotropy. Models of $\mathrm{D}^{0}$ flow with and without charm flow $^{36}$, represent the extreme cases and thus the expected measurement range. The corresponding distributions are shown in Figure 32. In the same figure, the $\mathrm{K}_{0} \mathrm{~V}_{2}$ is shown as a line. With these assumptions and the known efficiency, the estimated rates per event can be estimated. Assuming the desired statistical uncertainties are less than $10 \%$, the resulting required number of minimum bias events for the $\mathrm{D}^{0} v_{2}$ measurement is provided in Table 8 for various $\mathrm{p}_{\mathrm{T}}$ bins. As one can see, with 500 million events, the $\mathrm{D}^{0} \mathrm{v}_{2}\left(\mathrm{p}_{\mathrm{T}}\right)$ can be measured up to $\mathrm{p}_{\mathrm{T}} \sim 5 \mathrm{GeV} / \mathrm{c}$, using the proposed STAR HFT.

\begin{tabular}{|l|l|c|l|}
\hline $\mathbf{p}_{\mathbf{T}}(\mathbf{G e V} / \mathbf{c})$ & $\boldsymbol{\Delta}_{\mathbf{T}}(\mathbf{G e V} / \mathbf{c})$ & $\begin{array}{l}\text { \# of Events } \\
\mathbf{q}_{\mathbf{c}} \text { does flow }\end{array}$ & $\begin{array}{l}\text { \# of Events } \\
\mathbf{q}_{\mathbf{c}} \text { does not flow }\end{array}$ \\
\hline 0.6 & 0.2 & $260 \times 10^{6}$ & $525 \times 10^{6}$ \\
\hline 1.0 & 0.5 & $70 \times 10^{6}$ & $140 \times 10^{6}$ \\
\hline 2.0 & 0.5 & $53 \times 10^{6}$ & $125 \times 10^{6}$ \\
\hline 3.0 & 1.0 & $105 \times 10^{6}$ & $175 \times 10^{6}$ \\
\hline 5.0 & 1.0 & $210 \times 10^{6}$ & $440 \times 10^{6}$ \\
\hline
\end{tabular}

Table 8: An estimate of the number events required for $10 \%$ statistical errors for $D^{0}$ v2 measurements of $200 \mathrm{GeV} \mathrm{0-80 \%} \mathrm{Au} \mathrm{+} \mathrm{Au} \mathrm{collisions}\left(\mathrm{N}_{\text {bin }}=\mathbf{2 9 0}\right)$. A power-law shape $\mathrm{p}_{\mathrm{T}}$ distribution from the $p+p$ collision has been assumed with the mid-rapidity $d N / d y=0.03$ [Ref. 17]. The number of binary collisions is from the Glaubor calculations [Ref. 23].

As mentioned earlier, the assumptions in the $\operatorname{model}^{36}$ are extreme limits. In order to test the thermodynamic behavior of the D-meson, the most important region is $\mathrm{p}_{\mathrm{T}}<3 \mathrm{GeV} / \mathrm{c}$ because at higher $\mathrm{p}_{\mathrm{T}}$, other dynamical effects will become important (e.g. jet correlations). The proposed STAR HFT detector will be able to make precise $\mathrm{v}_{2}$ measurements in the transverse momentum region $0.7<\mathrm{p}_{\mathrm{T}}<3 \mathrm{GeV} / \mathrm{c}$. As we have done with the hadrons from the light-flavor sector ${ }^{5}$, the combined analysis of the D-meson spectra, $\mathrm{v}_{2}$ distributions and ratios will allow us to determine the charm quark collectivity and the thermodynamic nature of the medium created in heavy ion collisions at RHIC. 

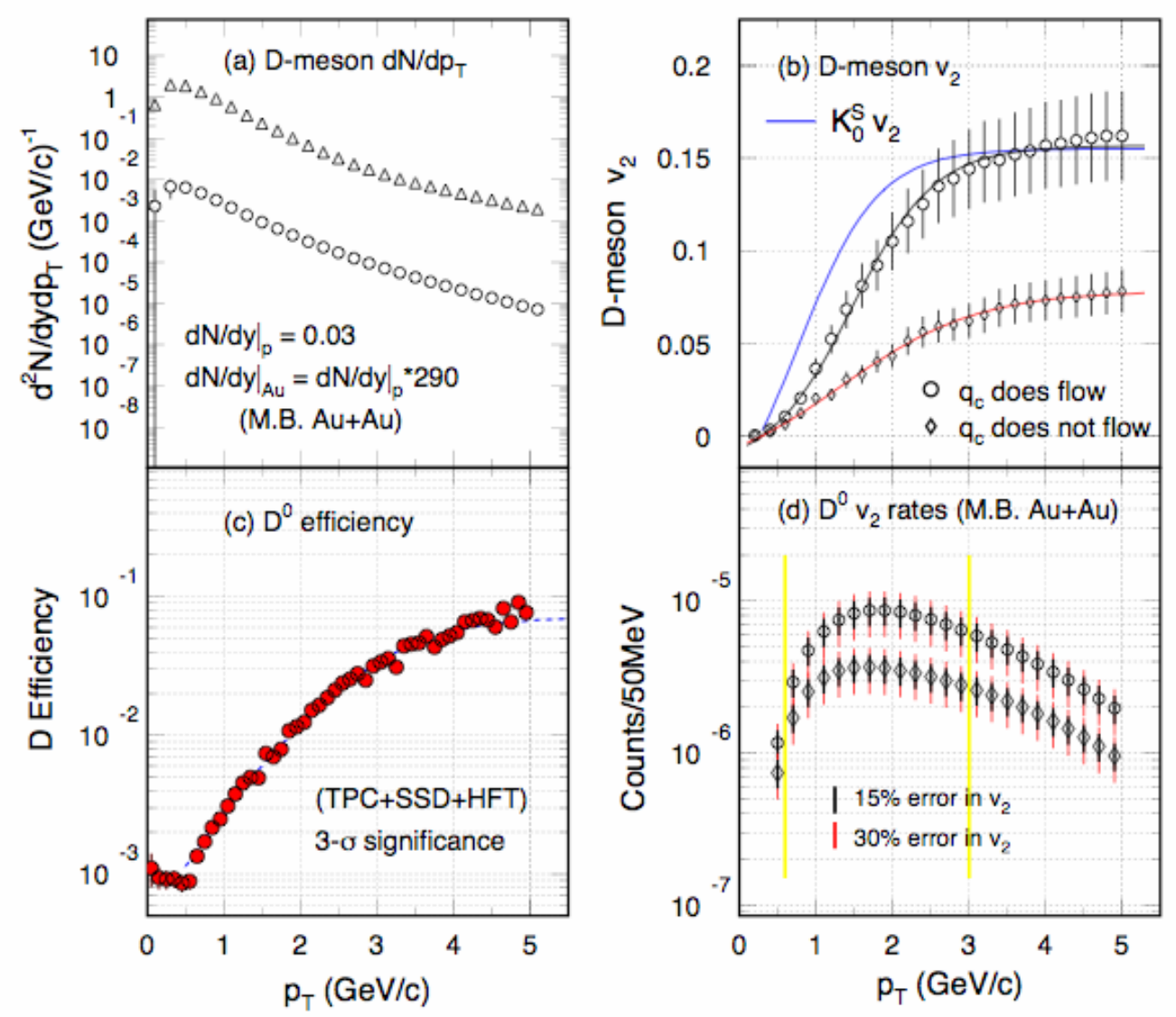

Figure 32: D-meson $v_{2}$ rate estimates: (a) $d N / d p_{T}$ distributions for $D$-meson. The integrated yield $\mathrm{dN} / \mathrm{dy}=\mathbf{0 . 0 3}$ as measured in $\mathrm{p}+\mathrm{p}$ collisions at $200 \mathrm{GeV}$ [Ref. 17]. The number of binary collisions $\mathrm{N}_{\mathrm{bin}}=\mathbf{2 9 0}$, corresponding to the minimum bias Au + Au collisions, is used to scale the Au $+\mathrm{Au}$ collisions. A power-law shape from $p+p$ collisions is assumed; (b) Assumed $v_{2}$ distributions for Dmesons. The solid-line is the results of fit to the measured Kaon $v_{2}$ [Ref. 23]. Both circle- and diamond-symbols are from [Ref. 46] for the case with and without charm quark flow, respectively. Error bars shown are from $15 \%$ systematic errors; (c) $3-\sigma$ significance $D^{0}$ efficiency with TPC+SSD+HFT. The Dashed-line is the fit to the simulated result; (d) $D^{0}$ meson $v_{2}$ rates from minimum bias $\mathrm{Au}+\mathrm{Au}$ collisions at $200 \mathrm{GeV}$. The small and large error bars are for $15 \%$ and $30 \%$ systematic errors, respectively. For the $v_{2}$ analysis, 12 bins in $\varphi$ are used.

\subsection{Reconstruction of the $\Lambda_{c}$ Baryon}

The $\Lambda_{\mathrm{c}}$ is the lowest lying heavy flavored baryon. Roughly $10 \%$ of the charm quarks end up in the baryon sector ${ }^{89}$ following a heavy ion collision. As has been mentioned in chapter 2 , the measurement of the $\Lambda_{c}$ is the best way for us to study heavy-flavored baryon/meson differences. It is important ingredient in our understanding of the hadrochemistry and hadronization involving heavy-flavors. The branching ratio of $\Lambda_{c}$ to the non-resonant decay is about 5\% with a $\mathrm{c} \tau=60 \mu \mathrm{m}$. With a similar reconstruction algorithm used in the previous sections of this proposal, we have simulated the reconstruction efficiencies for $\Lambda_{\mathrm{c}}$. 
Plots (a), (b) and (c) in Figure 33 are the dca distributions (distance of closet approach of the $\Lambda_{\mathrm{c}}$ to the primary vertex), decay length (the distance from primary vertex to the decay vertex), and $\cos (\theta)$ distributions (the angle between reconstructed $\Lambda_{c}$ momentum vector and the direction of the primary vertex), respectively. (See Figure 24.) The red-lines in Figure 33 are for primary charged tracks and the circles are for tracks from $\Lambda_{c}$ decay. The cuts on dca, decay length and $\cos (\theta)$ are $35<$ dca $<300 \mu \mathrm{m}, 50<$ decay length $<350$ $\mu \mathrm{m}$, and $\cos (\theta)>0.92$. Plots (d) and (e) are the invariant mass plots in the momentum windows: $2.0<\mathrm{p}_{\mathrm{T}}<2.2 \mathrm{GeV} / \mathrm{c}$ and $4<\mathrm{p}_{\mathrm{T}}<4.5 \mathrm{GeV} / \mathrm{c}$, respectively. The reconstructed efficiency is shown in plot (f). Additional work to further optimize the cuts, especially for the low $\mathrm{pT}$ region, is underway.

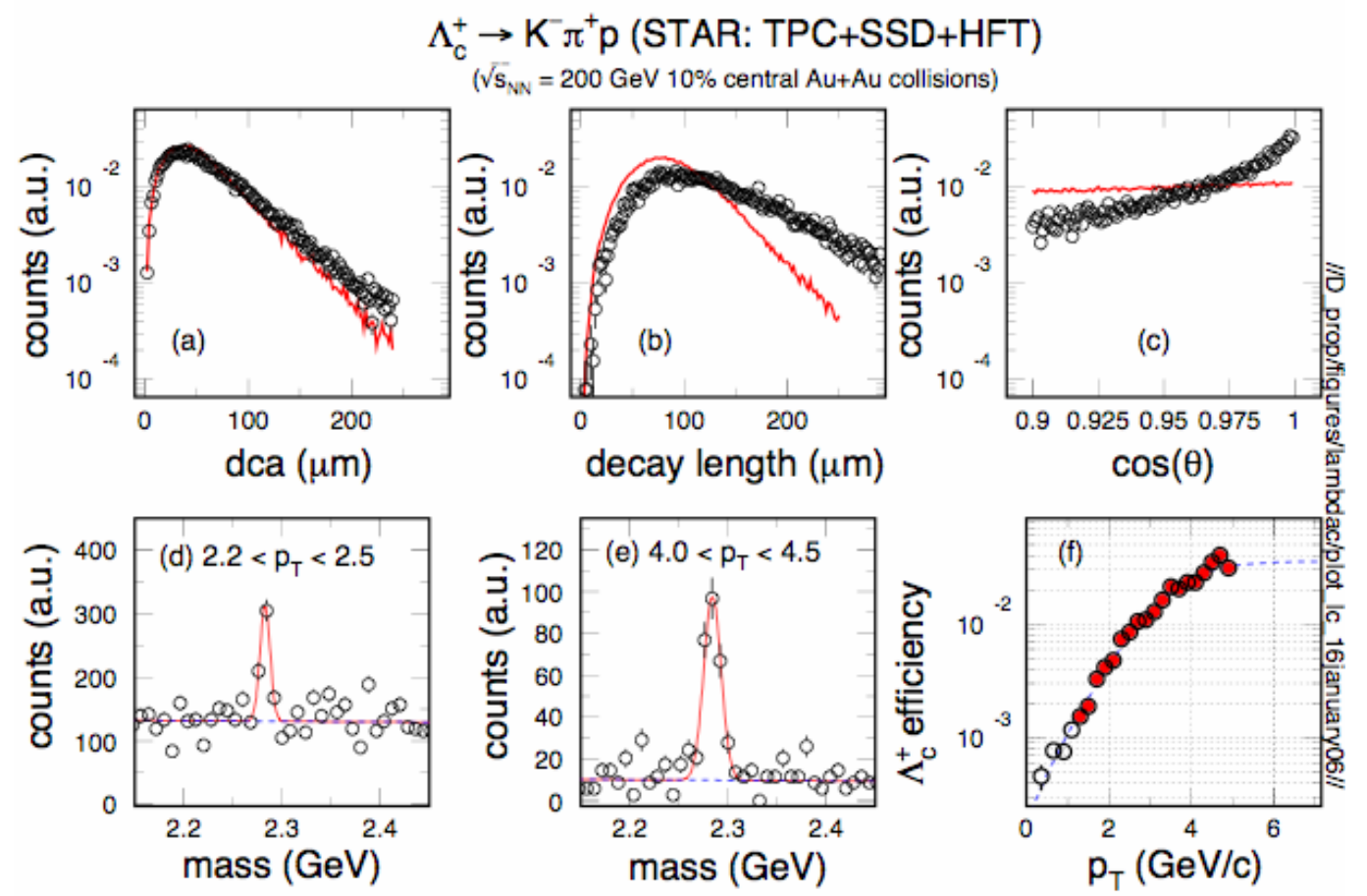

Figure 33: $\Lambda_{\mathrm{c}}$ decay length, dca, and invariant mass distributions.

In this study, an average of $0.8 \Lambda_{\mathrm{c}}$ per event was used. Below $\mathrm{p}_{\mathrm{T}}<2 \mathrm{GeV} / \mathrm{c}$, the signal over background ratio $\mathrm{S} /(\mathrm{S}+\mathrm{B})$ is less than 3. $50 \mathrm{M}$ central events (top $10 \%$ ) are needed in order to measure a $\Lambda_{\mathrm{c}} \mathrm{p}_{\mathrm{T}}$ distribution from $200 \mathrm{GeV} \mathrm{Au}+\mathrm{Au}$ collisions. Assuming binary scattering scaling for the heavy flavor production at $200 \mathrm{GeV}$, we find that $1000 \mathrm{M}$ peripheral events (60-80\%) will be required to measure the nuclear modification factor $\mathrm{R}_{\mathrm{AA}}\left(\mathrm{p}_{\mathrm{T}}\right)$ with an error of about $20 \%$ up to $\mathrm{p}_{\mathrm{T}} \sim 5 \mathrm{GeV} / \mathrm{c}$. This can then be used to compare with other heavy-flavor mesons and light-flavor hadrons. 


\subsection{Hit Density at the Front Surface of the HFT}

There are several issues that may affect the performance of the proposed HFT detector. The HFT is not triggered and is continually active, recording all particles that pass through it. This means that in tracking the events of interest there will be extraneous hits and pileup in the detector due to other collisions at an earlier or later time, tracks from beam gas showers, and other background sources. The areal density of extraneous hits that can be tolerated depends on the tracking precision projected onto the HFT surfaces and on details of the particle reconstruction algorithms; while the extraneous hit density depends on the HFT frame readout speed, beam luminosity, interaction cross sections and background rates from additional sources. Reasonable limits on these processes have been included in the simulations. The focus of this section is to discuss and quantify these and other background issues that go into the simulations.

To set the scale of the problem, consider the extraneous hit density from normal interactions. The parameters used in the calculation are shown in Table 9 and an estimate of the hit density is shown in Table 10 .

\begin{tabular}{|l|l|}
\hline $\mathrm{Au}+\mathrm{Au}$ Luminosity & $1 \times 10^{27} \mathrm{~cm}^{-2} \mathrm{~s}^{-1}$ \\
\hline $\mathrm{dN} / \mathrm{d} \eta$ (min bias) & 170 \\
\hline Min bias cross section & $10 \mathrm{barns}$ \\
\hline Interaction diamond size, $\sigma$ & $30 \mathrm{~cm}$ \\
\hline
\end{tabular}

Table 9: Luminosity and other parameters that determine the particle flux on the HFT.

\begin{tabular}{|l|l|l|}
\hline & HFT Outer Layer & HFT Inner Layer \\
\hline Radius & $5.0 \mathrm{~cm}$ & $1.5 \mathrm{~cm}$ \\
\hline Hit Flux & $5,600 \mathrm{~Hz} / \mathrm{cm}^{2}$ & $28,750 \mathrm{~Hz} / \mathrm{cm}^{2}$ \\
\hline Hit Density 4 ms Integration & $22.5 / \mathrm{cm}^{2}$ & $115 / \mathrm{cm}^{2}$ \\
\hline Projected Tracking Window Area & $0.6 \mathrm{~mm}^{2}$ & $0.15 \mathrm{~mm}^{2}$ \\
\hline HFT Hit Resolving Area & $0.001 \mathrm{~mm}^{2}$ & $0.001 \mathrm{~mm}^{2}$ \\
\hline Probability of HFT Pileup & $0.3 \%$ & $1 \%$ \\
\hline
\end{tabular}

Table 10: Integrated hit loading on the HFT and associated pileup.

As shown in the table, the accumulated hit density from the integrated $\mathrm{Au}+\mathrm{Au}$ collisions is $22.5 / \mathrm{cm}^{2}$ in the outer layer and $115 / \mathrm{cm}^{2}$ in the inner layer of the HFT ${ }^{90}$. This produces a pileup probability in the track search window of $14 \%$ and $17 \%$ at the inner and outer HFT barrels, respectively. The area of the track search window in this case is based on the projection resolution for $1 \mathrm{GeV} / \mathrm{c}$ pions as determined from GEANT simulations. For comparison, the unresolved hit pileup intrinsic to the HFT detector is much less, $0.28 \%$, and $1 \%$, for the outer and inner barrel respectively.

The luminosity applied in this case is the maximum instantaneous luminosity that was recorded during the $\mathrm{Au}+\mathrm{Au}$ run in $2004\left(1 \times 10^{27} \mathrm{~cm}^{-2} \mathrm{~s}^{-1}\right)$. This is 5 times the nominal 
"design luminosity". Design luminosity is defined to be $2 \times 10^{26} \mathrm{~cm}^{-2} \mathrm{~s}^{-1}$ averaged over a 10 hour fill. The STAR Experiment measures interaction rates through the zero-degree calorimeter detector, and a RHIC instantaneous luminosity of $2 \times 10^{26} \mathrm{~cm}^{-2} \mathrm{~s}^{-1}$ is equivalent to an instantaneous ZDC coincidence rate of $2000 \mathrm{~Hz}$. It should be noted that the RHIC beam luminosity starts high and drops with time over the course of a fill. During the RHIC II era, the most important improvements in RHIC luminosity will be longer decay times during the fill. However, we do expect an increase in peak luminosity by about a factor of 4, which will have the largest impact on our program. This is important, and so under RHIC II conditions we will have to improve the readout time of the HFT in order to limit pileup in the tracking search window to about $10 \%$.

Table 11 gives the same loading information resulting from a single central $\mathrm{Au}+\mathrm{Au}$ collision. The numbers for a single collision is a factor of 10 less then the integrated load, which demonstrates that an isolated collision is easily tracked by the HFT.

\begin{tabular}{|l|l|l|}
\hline & HFT Outer Layer & HFT Inner Layer \\
\hline Radius & $5.0 \mathrm{~cm}$ & $1.5 \mathrm{~cm}$ \\
\hline Hit Density $\mathrm{Au}+\mathrm{Au}$ Central Collision & $1.8 / \mathrm{cm}^{2}$ & $7.4 / \mathrm{cm}^{2}$ \\
\hline Projected Tracking Window Area & $0.6 \mathrm{~mm}^{2}$ & $0.15 \mathrm{~mm}^{2}$ \\
\hline HFT Hit Resolving Area & $0.001 \mathrm{~mm}^{2}$ & $0.001 \mathrm{~mm}^{2}$ \\
\hline Probability of HFT Pileup & $0.02 \%$ & $0.09 \%$ \\
\hline
\end{tabular}

Table 11: Hit loading on the HFT from Au + Au central collisions and associated pileup.

\subsubsection{Measured Hit Density at $6 \mathrm{~cm}$ Radius in STAR}

We have used the STAR SVT to actually measure the hit density that will contribute to pileup in the HFT. The SVT is the innermost detector currently available in STAR and it gives the best-hit density information closest to the interaction point. This is of interest because it can identify potential background contributions including very low momentum tracks. As it turned out the measured hit density at the SVT inner layer for central collision triggers is $1 \mathrm{hit} / \mathrm{cm}^{2}$. This is consistent with the expected track density for central $\mathrm{Au}+\mathrm{Au}$ collisions with $\mathrm{dN} / \mathrm{d} \eta=700$, so we see no evidence of additional background beyond the pileup of normal $\mathrm{Au}+\mathrm{Au}$ events ${ }^{91}$. This measurement was done at $\mathrm{B}=0.25 \mathrm{~T}$, half the normal STAR magnet field, to open the acceptance to lower momentum particles. It would be desirable to use SVT events with a zero bias trigger to have a measure of other background sources such as beam gas showers, but so far, our noise suppression filters for the SVT are not sufficient for this task.

Although we do not have a direct measure of beam showering, we do know from studies of space charge distortions in the STAR TPC that at larger radii, $65 \mathrm{~cm}$ to $200 \mathrm{~cm}$, the ionization density due to normal $\mathrm{Au}+\mathrm{Au}$ interactions is larger than the contributions from other sources such as beam gas ${ }^{92}$ interactions. 


\section{CMOS Sensors}

\subsection{Introduction}

The STAR heavy flavor physics program requires a thin, fast, detector that can operate in a relatively high radiation environment. Our goal is to measure charmed hadron decays and to measure flow in the charm sector. Since flow is a soft process, the HFT must measure transverse momenta down to $150 \mathrm{MeV} / \mathrm{c}$. To reach this low threshold requires a very thin detector in order to maintain precise tracking without degradation by multiple Coulomb scattering. It is difficult to meet all of these requirements using the "usual" techniques currently employed in high energy physics experiments.

One possible technology choice for a vertex detector is Charged Coupled Devices (CCD). They provide the necessary granularity and can be thinned down to the desired thickness, but these detectors are slow, radiation sensitive and complex to operate. On the other hand, Hybrid Pixel Sensors, and other sensor concepts, are fast and radiation tolerant but suffer from modest granularity problems; also, they are usually too thick for our application.

The emergence of CMOS sensor technology offers a new perspective on high precision charged particle tracking and vertex finding. This technology can provide the performance parameters required by the HFT. Recent developments have shown that CMOS technology is capable of excellent spatial resolution and charge collection efficiency, together with satisfactory radiation tolerance.

\subsubsection{Choice of technology}

Lets look at these technology choices in more detail. At this time, there are four good technologies that can be used for a thin vertex detectors: Charge Coupled Devices (CCD), Active Pixel Sensors (APS), Hybrid Pixels, and DEPleted Field Effect Transistor structure (DEPFET). Each of these devices has strengths and weaknesses. In the process of preparing this proposal, we have evaluated each and selected APS as our choice for the HFT detector.

\section{CCDs}

At SLAC, the SLD collaboration built and successfully operated a pixel vertex detector ${ }^{93}$, VXD3, based on CCD technology. ${ }^{94}$ But since silicon is damaged by radiation and CCDs require that the charge be transferred from one pixel to another, CCDs are more susceptible to radiation than other vertex devices. Charge in the end row of a CCD chip, for example a $1000 \times 1000$ array, must be transferred through more than 1000 pixels before being digitized. Therefore, any small loss in charge transfer produces large signal losses and signal sharing. The SLD vertex detector ran at a relatively low radiation 
intensity because that is the nature of the SLAC Linear electron Collider (SLC), so the SLC CCD could tolerate the radiation environment. In addition, the complexity of the clocking makes the readout slow. This was suitable for the SLC where it only had to be operated at $2 \mathrm{~Hz}$.

CCDs require significant power to clock the charge around the chip because high capacitance electrode structures covering the whole chip must be voltage switched. This becomes a power versus speed trade off with consequences in the mass budget because liquid cooling is usually required. In any case, cooling is a complication for CCD operation. For instance, VXD3 used $\mathrm{LN}_{2}$ gas to cool the device but because the ladders were operated far below room temperature, elaborate mechanical and alignment systems were needed to achieve the excellent resolution of about $30 \mu \mathrm{m}$ for determining the impact parameter. An outer heated jacket was needed to prevent condensation and the cooling system added extra complication and mass.

Finally, to fabricate CCDs requires a very special process. This process requires specialized knowledge, is expensive, and requires a long learning curve to become familiar with its details. Currently, there is a group studying whether the limitations of the process can be overcome to use this technology it at a future electron collider. ${ }^{95}$

\section{Hybrid Pixels}

The radiation environment at the LHC is much higher. For this reason, and the need to have the vertex detector in the trigger, CCDs are not an appropriate choice at the LHC (Large Hadron Collider) ${ }^{96}$ at CERN, which has a much higher radiation background than SLC. At the LHC, the three major experiments decided to use a hybrid technology where the sensor is bump bonded to a read-out chip. The hybrid technology has the disadvantage that the pixel size is much greater than a CCD pixel and two chips have to be layered on top of each other. The two chips and their interconnection are much thicker than can be done in CCD technology.

\section{DEPFET}

The MPI Semicondcutor Laboratory has recently invented ${ }^{97}$ and has been the pioneering leader in the use of DEPFETs. ${ }^{98}$ This concept is based on the combination of the sideward depletion, as used in a semiconductor drift detector with a field effect transistor to collect the charge. The MPI Group has a conceptual design for a detector for the Linear Collider but much development is needed to make a realistic device. DEPFETs require a very special process and MPI is the only producer of this device so any development must be done within that institute. (This has both the advantage and disadvantage that its process is controlled by physicists.) 


\begin{tabular}{|c|c|c|c|c|c|c|}
\hline Chip & Year & Process $(\mu \mathrm{m})$ & $\begin{array}{l}\text { Epi. } \\
(\mu \mathrm{m})\end{array}$ & \begin{tabular}{|l} 
Pitch \\
$(\mu \mathrm{m})$
\end{tabular} & Pixels & Comments \\
\hline MIMOSA-1 & 1999 & AMS 0.6 & 14 & 20 & $4 \mathrm{k}$ & thick epitaxial layer \\
\hline MIMOSA-2 & 2000 & MIETEC 0.35 & 4.2 & 20 & $4 \mathrm{k}$ & thin epitaxial layer \\
\hline MIMOSA-3 & 2001 & IBM 0.25 & 2 & 8 & $32 \mathrm{k}$ & deep sub-mm \\
\hline MIMOSA-4 & 2001 & AMS 0.35 & no & 20 & $4 \mathrm{k}$ & low dopant substrate \\
\hline MIMOSA-5 & 2001 & AMS 0.6 & 14 & 17 & $1 \mathrm{M}$ & real scale $1 \mathrm{M}$ pixels \\
\hline MIMOSA-6 & 2002 & AMIS 0.35 & 4.2 & 28 & $3 \mathrm{k}$ & $\begin{array}{l}\text { fast column parallel readout } \\
\text { internal data sparsification }\end{array}$ \\
\hline MIMOSA-7 & 2003 & AMS 0.35 & no & 25 & $1 \mathrm{k}$ & $\begin{array}{l}\text { fast column parallel readout } \\
\text { internal data sparsification. } \\
\text { (photoFET) }\end{array}$ \\
\hline MIMOSA-8 & 2003 & TSMC 0.25 & $\sim 8$ & 25 & $4 \mathrm{k}$ & $\begin{array}{l}\text { fast column parallel readout } \\
\text { internal data sparsificaton }\end{array}$ \\
\hline MIMOSA-9 & 2004 & $\begin{array}{l}\text { AMS } 0.35 \\
\text { opto }\end{array}$ & $\sim 14$ & $\begin{array}{l}20 \\
30 \\
40\end{array}$ & $7 \mathrm{k}$ & tests diodes/pitch/leakage current \\
\hline $\begin{array}{l}\text { MIMOSA-9 } \\
\text { (no epi) }\end{array}$ & 2004 & $\begin{array}{l}\text { AMS } 0.35 \\
\text { opto }\end{array}$ & no & $\begin{array}{l}20 \\
30 \\
40\end{array}$ & $7 \mathrm{k}$ & tests diodes/pitch/leakage current \\
\hline $\begin{array}{l}\text { MIMOSA-10 } \\
\text { (MIMOSTAR-1) }\end{array}$ & 2004 & TSMC 0.25 & $\sim 8$ & 30 & $16 \mathrm{k}$ & first prototype for STAR HFT \\
\hline MIMOSA-11 & 2005 & $\begin{array}{l}\text { AMS } 0.35 \\
\text { opto }\end{array}$ & $\sim 14$ & 30 & $7 \mathrm{k}$ & radiation tolerant structure \\
\hline $\begin{array}{l}\text { MIMOSA-12 } \\
\text { (Mosaic 1) }\end{array}$ & 2005 & $\begin{array}{l}\text { AMS } 0.35 \\
\text { high resistive }\end{array}$ & no & 35 & $0.6 \mathrm{k}$ & multi-memory pixels (FAPS) \\
\hline $\begin{array}{l}\text { MIMOSA-13 } \\
\text { (Mosaic 2) }\end{array}$ & 2005 & $\begin{array}{l}\text { AMS } 0.35 \\
\text { high resistive }\end{array}$ & no & 20 & $1.4 \mathrm{k}$ & fast column readout \\
\hline $\begin{array}{l}\text { MIMOSA-14 } \\
\text { (MIMOSTAR-2) }\end{array}$ & 2005 & $\begin{array}{l}\text { AMS 0.35 } \\
\text { opto }\end{array}$ & no & 30 & $16 \mathrm{k}$ & second prototype STAR HFT \\
\hline MIMOSA15 & 2005 & $\begin{array}{l}\text { AMS } 0.35 \\
\text { opto }\end{array}$ & $\sim 14$ & $\begin{array}{l}20 \\
30\end{array}$ & $7 \mathrm{k}$ & multi-purpose tracker-imager \\
\hline SUC 1 & 2003 & AMIS 0.35 & 4.2 & $\begin{array}{l}25 \\
35\end{array}$ & $4 \mathrm{k}$ & $\begin{array}{l}\text { radiation tolerant structure } \\
\text { (SUCIMA project) }\end{array}$ \\
\hline SUC 2 & 2003 & AMS 0.35 & no & 40 & $2 \mathrm{k}$ & $\begin{array}{l}\text { low dopant substrate } \\
\text { (SUCIMA project) }\end{array}$ \\
\hline SUC 3 & 2003 & AMIS 0.35 & 4.2 & 20 & $8 \mathrm{k}$ & $\begin{array}{l}\text { radiation tolerant structure } \\
\text { (SUCIMA project) }\end{array}$ \\
\hline SUC 4 Mtera & 2004 & AMS 0.35 & 14 & 150 & $12.5 \mathrm{k}$ & $\begin{array}{l}\text { Hadron therapy/beam monitor. } \\
\text { (SUCIMA project) }\end{array}$ \\
\hline SUC 5 & 2004 & AMIS 0.35 & 4.2 & 30 & $65 \mathrm{k}$ & $\begin{array}{l}\text { proton dosimetry } \\
\text { (SUCIMA project) }\end{array}$ \\
\hline
\end{tabular}

Table 12: Table - Chart of APS chips that the IReS group has produced in the past 5 years [99].

\section{Active Pixel Sensors}

APS devices have been used as photon detectors since late 1960s. ${ }^{100}$ They have recently surpassed CCDs in the photography market because of their lower cost and lower demand for power. Power consumption is important in a particle detector application 
because a detector that can be air cooled is thinner and lower mass, by definition, than a detector that requires water cooling. The IReS group in Strasbourg France has done a great deal of research on these detectors; taking them from small prototypes to large arrays of successful detector elements. Table 12 (above) presents a detailed listing of the CMOS sensor program.

APS technology is our preferred technology for the HFT and we are working with the Strasbourg group to design and utilize this technology in our detector. The APS technology will be described in more detail in the next section.

\subsection{Main Features and Performance of CMOS Active Pixel Sensors}

CMOS sensors are manufactured using industry-standard CMOS technology. This offers low fabrication costs and fast turn-around times in their development. The key element of this technology, for our purposes, is the use of an n-well/p-epi diode to collect the charge, through thermal diffusion, which is generated by the impinging particles in the thin epitaxial layer underneath the read-out electronics ${ }^{101}$ (See Figure 34). An attractive feature of these sensors is that they allow fabrication of System-on-Chips ( $\mathrm{SoC}$ ) by integrating signal processing micro-circuits (amplification, pedestal correction, digitization, discrimination, etc.) on the detector substrate. Moreover, a CMOS substrate can be thinned down to a few tens of microns because the active region is less than 20 $\mu \mathrm{m}$ thick.

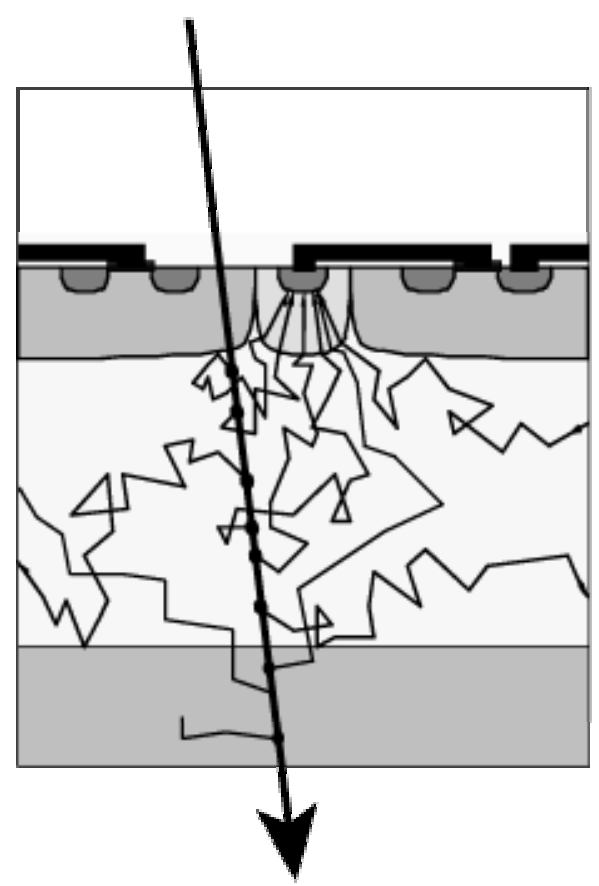

Figure 34: Epitaxial Silicon used as a sensor. In this design, a primary ionizing particle creates free charges in the epitaxialy grown $\mathrm{Si}$ layer that is a few tens of microns thick and a few electrons in the bulk layers. The liberated charges are then free to diffuse towards a potential well structure at the top of the sensor where they are extracted and readout into a DAQ system.

The ability of these sensors to provide charged particle tracking is now well established. $^{102}$ The IReS group, at Strasbourg, has built a series of these sensors, which 
they called MIMOSA. ${ }^{103}$ Similarly, the LBL/UCI group has also built and successfully tested these sensors. The MIMOSA line of detectors has explored different CMOS fabrication processes and key parameters of the charge sensing system, and the results demonstrate that a detection efficiency of $\sim 99 \%$ and a single point resolution of $\sim 2 \mu \mathrm{m}$ can be achieved using a pixel pitch of $20 \mu \mathrm{m}$. The prototypes also show that digitizing the charge with a small number of ADC bits does not degrade the resolution significantly (the measured reduction was $\sim 2.5-3 \mu \mathrm{m}$ ) while the double hit resolution is $\sim 30 \mu \mathrm{m}$.

The radiation tolerance of the sensors to bulk damage ${ }^{104}$ was also investigated. No significant performance loss was observed up to fluences close to $10^{12} \mathrm{n}_{\mathrm{eq}} \mathrm{cm}^{-2}$. As far as ionizing radiation damage is concerned, the real potential of this technology is still being explored, but it is already established that it stands up to more than $100 \mathrm{kRad}$.

Most of the R\&D at IReS was performed with small prototypes (a few $\mathrm{mm}^{2}$ ) containing a few thousand pixels. Figure 35 shows a full size prototype (i.e. $\sim 3.5 \mathrm{~cm}^{2}$ ) called MIMOSA-5. It is composed of $\sim 1$ million pixels per chip, and it was fabricated on a 6 inch wafer, as shown in the figure. The wafers were thinned down to $120 \mu \mathrm{m}$ before the chip was cut and diced into individual, reticle sized, detector elements.

Tests at the CERN-SPS confirmed that MIMOSA-5 performed as well as the smaller prototypes: a 99\% detection efficiency was observed with $\sim 2 \mu \mathrm{m}$ single point resolution. The prototypes were operated with a read-out time of $25 \mathrm{~ms}$, which was limited by the maximum operation frequency of the read-out board (i.e. $10 \mathrm{MHz}$ ). The chip was actually designed for a 4 times faster read-out speed. The LBL group has tested several of these chips at the LBL ALS and have measured the expected Landau spectrum on each.

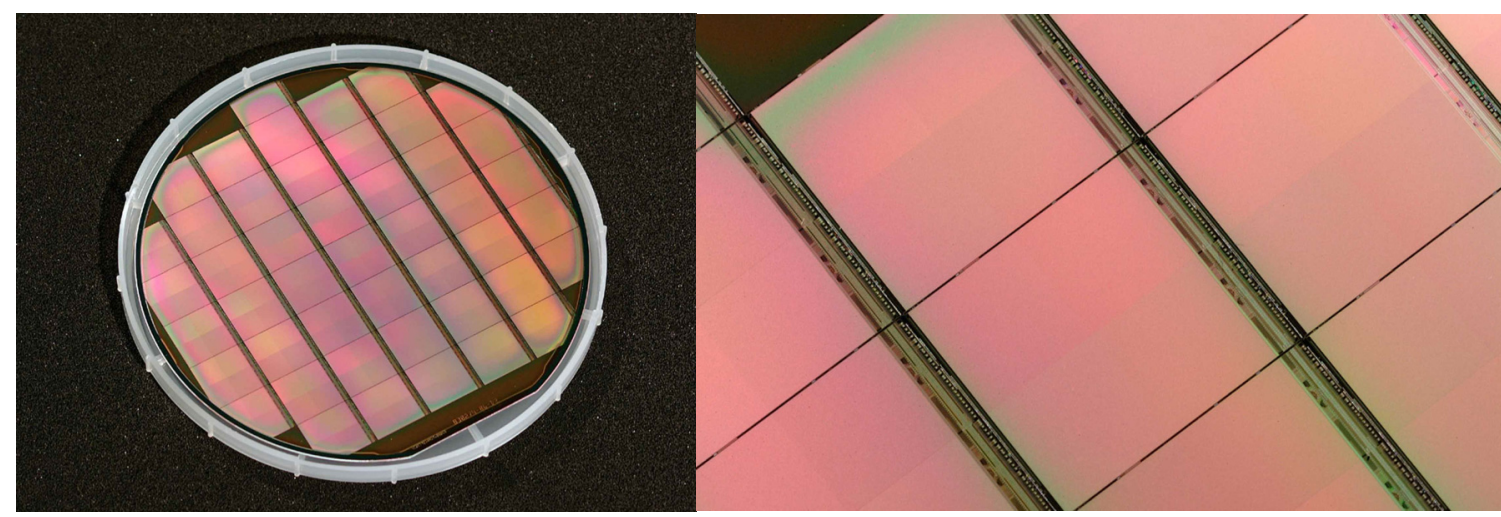

Figure 35: Wafer of reticle size sensors (left) and zoomed-in view of individual chips (right).

After MIMOSTAR-5, several fabrication processes were explored, aiming to find the process providing the smallest leakage current. In general, several parameters underlying the sensor performance parameters depend on features specific to each fabrication process and so the process specific characteristics need to be explored in parallel with the development of the chip architecture. 
For instance, a new fabrication technology, relying on a lightly doped substrate but exhibiting no epitaxial layer was investigated with two different prototypes chips. Further IReS tests show that a detection efficiency of $99.9 \%$ can be achieved with this technology, as well as a single point resolution of about $2.5 \mu \mathrm{m}^{105}$. A major advantage of this technology is that a large signal can be generated because the charges are collected from several tens of microns of Si instead of from $\sim 10 \mu \mathrm{m}$ in an epitaxial layer. The extra charge makes it well suited to applications with substantial electronic noise.

\subsection{Thinning}

Up to now, tested MIMOSA-5 chips have been thinned down to $120 \mu \mathrm{m}$ thickness. This operation was successful and we have not found any degradation of the sensor parameters.

In the HFT, the MIMOSTAR thickness will be $50 \mu \mathrm{m}$. Thinning a 6 or 8 inch wafer down to this thickness is not expected to be a problem, since the process is an industry standard technique. We have demonstrated that $50 \mu \mathrm{m}$ sensors are mechanically stable and can be assembled into ladders. We have used low-yield MIMOSA-5 wafers to make these investigations and have successfully thinned detectors to $50 \mu \mathrm{m}^{106}$.

Our colleagues at the LBL light source have just characterized several MIMOSA-5 chips in the ALS's $1.5 \mathrm{GeV} / \mathrm{c}$ electron beam and have subsequently thinned them. Tests of the thinned chips are in progress.

\subsection{Additional R\&D}

CMOS sensors have been developed in Strasbourg since 1999 for various applications, which range from vertex detectors for subatomic physics, to bio-medical imaging (e.g. beam monitoring for oncotherapy, dosimeters for brachyotherapy) and operational dosimetry (e.g. control of ambient radon and neutron radiation levels in nuclear plants).

Several application domains call for SoCs providing fast read-out speed (meaning signal treatment and data flow reduction integrated on the chip), high radiation tolerance, minimal material budget and low power dissipation. Developments for the STAR upgrade will thus benefit from the synergy with the R\&D for other applications, in terms of fabrication process exploration, development of fast signal processing architectures, radiation tolerance investigations, and improvements. More information on the activities and achievements of the Strasbourg research team are available in Ref. [107].

Starting with a IReS design, the LBNL/UCI group has built several generations of APS sensors. These devices were built in the $0.25 \mu \mathrm{m}$ process at TSMC. These ICs have been

tested using different sources, i.e. ${ }^{55} \mathrm{Fe}, 1.5 \mathrm{GeV}$ accelerator electrons and a scanning 
electron microscope. Figure 36 shows one of the sensors that has 16 different test structures.

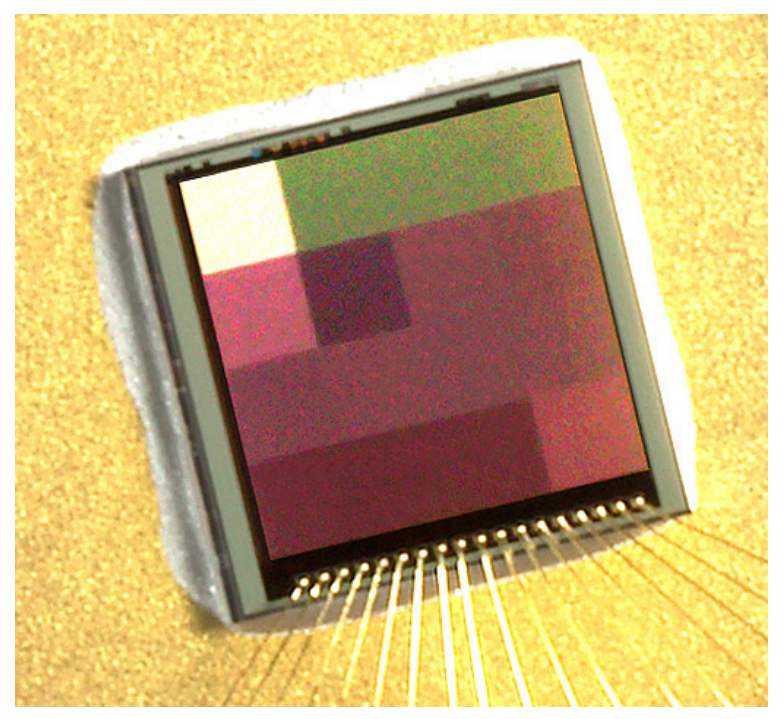

Figure 36: An APS Sensors developed by the LBNL/UIC group. The picture shows 16 separate test structures. Each structure has a $36 \times 36$ array of $20 \mu \mathrm{m}$ pixels.

Our measurements have confirmed the IReS results that APS sensors can measure charged particles with excellent spatial resolution ${ }^{108,109}$. Figure 37 shows several 1.5 GeV electrons recorded at LBNL's Advanced Light Source.

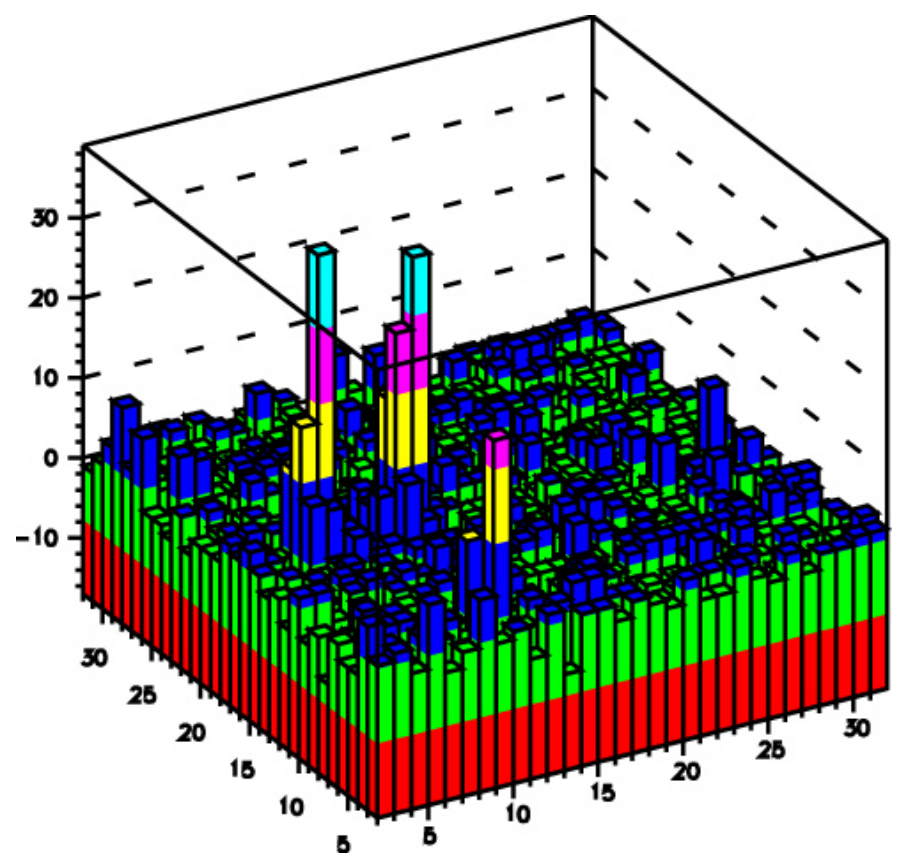

Figure 37: This graph shows the results of one event taken with $1.5 \mathrm{GeV}$ electrons. Each bin represents one pixel and the height is proportional to the measured charge. Several electron hitselectrons can be identified in the plot. 
To study the effects of radiation, we have exposed the chips to protons at LBNL's 88 " Cyclotron. Afterwards, we measured the change in leakage current and pulse height. These results show that there was a modest change with an irradiation of $300 \mathrm{krad}$. What was most interesting is that after 6 months, the device self annealed. Therefore, the effect of gradual radiation is much less than that of an acute exposure ${ }^{110}$. These radiation measurements are complementary to the neutron exposures measured by the IReS group.

We also completed a study on the effects of varying the pixel pitch. We built and tested a sensor with 5, 10, 20 and $30 \mu \mathrm{m}$ spacing. Our tests show that to first order the charge collection was identical when comparing the central pixel to the charge collected on its neighbors. ${ }^{111}$ As the pixel spacing decreases, more total charge is collected. This observation can be explained by the fact that as pixel spacing becomes smaller, the charge is collected by the diode faster, so there is less time for it to recombine. This result implies that we can easily extrapolate our measurements at $20 \mu \mathrm{m}$ spacing to the selected spacing of $30 \mu \mathrm{m}$ for MIMOSTAR.

Our group has been looking at several other techniques to improve APS sensors. It is clearly desirable to speed up the readout as well as reduce the signal spreading to multiple pixel diodes. Concentrating the signal onto a single diode would improve signal to noise.

We can increase the fraction of charge collected by a single pixel using the photo-gate technology. In principle, this technology allows us to use a large area photo-gate for charge collection, without increasing the capacitance, because the charge is transferred from the photogate region to a low capacitance diode. Even though we have demonstrated the sensitivity of the photo-gate structure to X-rays, we have not achieved the expected performance of the device. The transfer time required for moving electrons from the photo-gate to the drain appears to be very long (several $\mathrm{ms}$ ). We believe this undesirable signal delay is probably consistent with surface traps at the $\mathrm{Si}_{2}$ silicon boundary. Studies to find a way to avoid this delay are in an SBIR proposal that is currently being considered.

Correlated double sampling (CDS) is a standard technique that is used to remove the fixed pattern noise and KTC noise introduced by the reset transistor. Its main drawback is the required read out and storage of a full frame of data. To avoid doing CDS, we have produced a clamp circuit ${ }^{12}$ that reduces the reset noise by a factor of 3 .

Two generations of "active reset chips" have been fabricated and tested. In this approach the pixel voltage is reset to the empty level with a feed back amplifier potentially reducing fixed pattern noise and the KTC noise associated with a passive reset switch. Preliminary testing shows some noise reduction, but not the full potential improvement. We will be making some changes to this circuit and fabricating this in a new sensor design. 
To explore how charge is collected on a sensor as a function of position, we tested a device at LBNL's National Center for Electron Microscopy. Using the scanning electron microscope, we have been able to position the beam on a pixel with precision of about 1 $\mu \mathrm{m}$. This allows us to explore algorithms for determining the position of an incoming particle by relating how charge is shared among neighboring pixels. The results show that we can easily obtain the position of the incoming electron by weighting the charge collected from nearby pixels.

We have also been collaborating with colleagues at UC San Diego on the suitability of using an APS sensor in an electron microscope ${ }^{113}$. We have been studying the response of electrons from energies of $100 \mathrm{keV}$ to $300 \mathrm{keV}$. Results show that single electrons can be detected with a good signal to noise ratio. This technique is well suited for this application as it overcomes problems for traditional CCDs that suffer radiation damage. Excellent pictures of proteins have been taken. Using APS devices are very appealing for this different type of application.

We have several ideas on how to further improve our device. We have fabricated a sensor with different diode sizes. We have used this device to understand the effect of diode size on signal to noise. The results show that the smallest diode that meets the design rules produces the best signal to noise.

Recently, we submitted an IC design without an epi-layer. We have received the sensor and in the process of testing it. Simulations show that we should be able to collect more charge from the process.

In addition, we are studying ways to reduce the sampling time. In the current MIMOSTAR design, the sensor is always sensitive to radiation. We will study a sampleand-hold circuit to see if we can reduce the time window of sensitivity. If we could gate such a sensor, then pile-up effects of out of time interactions would be significantly reduced.

\subsection{MIMOSTAR Sensor Design}

Based on our experience with CMOS technology, a new series of chips, MIMOSTAR, have been fabricated. The first chip in the series is also called MIMOSTAR-10, which indicates its place in the evolution tree of CMOS sensors. Its most significant design parameters are:

- Pixel pitch: $30 \mu \mathrm{m}$.

- Passive forward bias diode in place of reset switch

- Additional details can be found in Ref. [114]. 
The goal of the MIMOSTAR series is to provide for a full-scale prototype that is suitable for evaluating the performance of a CMS sensor in a collider environment.

Since the read-out speed requirement for this prototype is modest, the chip's architecture is based on relatively slow signal processing at the pixel level. This low speed requirement enables us to quickly implement this sensor, while at the same time we are independently developing a full-featured sensor for RHIC II. Its design favors moderately low noise and modest power consumption. On the other hand, high speed signal processing is needed at the chip level (i.e. after amplification and multiplexing) due to the need for short integration times and limited shot noise.

The chip includes JTAG based remote control functionality (e.g. bias setting, test settings, etc.). The power dissipation of this architecture is estimated to be slightly more than $50 \mathrm{~mW}$ (i.e. less than $15 \mathrm{~mW} / \mathrm{cm}^{2}$ ), which can easily be cooled with air.

The IReS group has studied MIMOSTAR-1, which was fabricated in the TSMC $0.25 \mu \mathrm{m}$ process. They showed that the conventional controls work (via the JTAG controller), and that the bias circuits work and exhibit linear response via their DACs. The analog performance of the chips has been tested and the gain on the pre-amplifiers was about 3.5 at the two $10 \mathrm{MHz}$ outputs. However, due to the inherent properties of the TSMC process, the charge collected on the n-diode could not hold the collected charge long enough for it to be read out. Our group at Berkeley Lab observed a similar effect with another chip designed in collaboration with UCI, which also was fabricated in the TSMC 0.25 process.

Consequently, we decided to switch to the AMS 0.35 OPTO process, in which the passive forward bias diode reset circuit has already been demonstrated to work. A new chip, MIMOSTAR-2, has been fabricated, passed ${ }^{55} \mathrm{Fe}$ tests, and then tested in DESY's 5 $\mathrm{GeV}$ electron beam. A radiation tolerant design and a standard design showed an efficiency greater than $99.7 \%$ at a temperature of $40 \mathrm{C}$. The radiation tolerant design showed a very small increase in noise at $40 \mathrm{C}$ when it was exposed to $23 \mathrm{kRad}$ of ${ }^{60} \mathrm{Co}$.

The specifications used to design the chip are shown in Appendix I-MIMOSTAR II Specifications and a detailed guide to using the chip has been completed and is included in Appendix II-MIMOSTAR II Users Guide.

\subsection{The path to a CMOS detector for the HFT}

With this research and development done at IReS and LBL, we are confident that we can build a CMOS sensor appropriate for RHIC II luminosities. To achieve this goal, we have identified a series of steps that must be taken before we can design and build the final HFT sensor. In the next subsections, we will describe the different R \& D steps necessary to achieve this ultimate goal. 


\subsubsection{MIMOSTAR-3 - a half sized chip}

MIMOSTAR-3, which is half the size of the final sensor, continues the work done on MIMOSTAR-2. It is made up of $640 \times 320$ pixels. The fabrication will rely on an engineering run in AMS 0.35 OPTO technology. The chip has two analog outputs implemented on the same side of the sensor. Each output runs in parallel at $50 \mathrm{MHz}$ so the total time for readout is $2 \mathrm{~ms}$.

Once these chips have been tested, they will be incorporated on a ladder for a full function test in STAR. As a ladder will be half the final size, significant tests will be able to be done.

\subsubsection{MIMOSTAR-4 - a full sized chip}

Using the experience gained from building and testing MIMOSTAR-3, we will design and manufacture a full sized sensor that can both be used in a full sized ladder prototype. This chip will be twice the size of MIMOSTAR-3, so it will be $640 \times 640$ pixels. It will have 2 analog outputs on one side running at the same speed of MIMOSTAR-3, so it will take $4 \mathrm{~ms}$ to read out all of the pixels. The fabrication of this chip will be done in a production run.

Table 13 shows how the measured performance of the MIMOSA-5 sensor compares to the specifications of MIMOSTAR-4. Most of the requirements (granularity, radiation tolerance, thinning, read-out speed, power dissipation, and sensor size) have already been demonstrated with MIMOSA-5. Some effort is still needed to achieve a higher read-out speed and higher yield during thinning. Moreover, since the sensors will be operated at room temperature, special attention will be given to the magnitude of the leakage current in order to keep the corresponding shot noise at an acceptable level. 


\begin{tabular}{|l|l|l|}
\hline \multicolumn{1}{|c|}{ Parameter } & \multicolumn{1}{c|}{$\begin{array}{c}\text { MIMOSTAR-4 } \\
\text { specifications }\end{array}$} & \multicolumn{1}{|c|}{ MIMOSA-5 performance } \\
\hline Detection efficiency & $>98 \%$ at $30-40^{\circ} \mathrm{C}$ & $\sim 99 \% \leq 20^{\circ} \mathrm{C}$ \\
\hline Single point resolution & $<10 \mu \mathrm{m}$ & $\sim 2 \mu \mathrm{m}$ \\
\hline Granularity (pixel pitch) & $30 \mu \mathrm{m}$ & $17 \mu \mathrm{m}$ \\
\hline Read-out time & $4 \mathrm{~ms}$ & $24 \mathrm{~ms}(<20 \mathrm{~ms}$ possible $)$ \\
\hline Ionizing radiation tolerance & $3.7 . \mathrm{kRad} / \mathrm{yr}^{\dagger}$ & $>100 \mathrm{kRad}$ \\
\hline Fluence tolerance & $2 \times 10^{10} \mathrm{n}_{\mathrm{eq}} / \mathrm{cm}^{2}$ & $\leq 10^{12} \mathrm{n}_{\mathrm{eq}} / \mathrm{cm}^{2}$ \\
\hline Power dissipation & $<100 \mathrm{~mW} / \mathrm{cm}^{2}$ & $\sim 10 \mathrm{~mW} / \mathrm{cm}^{2}$ \\
\hline Chip size & $\sim 2 \times 2 \mathrm{~cm}^{2}$ & $1.9 \times 1.7 \mathrm{~cm}^{2}$ \\
\hline Chip thickness & $\sim 50 \mu \mathrm{m}$ & $120 \mu \mathrm{m}$ \\
\hline
\end{tabular}

Table 13: Comparison between MIMOSA-5 characteristics and MIMOSTAR-4 specifications.

\subsubsection{The ultimate sensor}

A next generation CMOS IC is needed to meet the requirements of the high intensity at RHIC II. At these higher luminosities, we need to read-out the chip to match the $1 \mathrm{~ms}$ readout time of the TPC and to have the chip sensitive for a much shorter time to reduce the effect of pileup. The goal of our R\&D program is to find a sensor that can meet the specifications listed in Table 14.

This sensor will be the same size as MIMOSTAR-4. It will have the same pitch, $30 \mu \mathrm{m}$, and pixel array, $640 \times 640 \mu \mathrm{m}$. It also will be thinned to $50 \mu \mathrm{m}$. A specific architecture needs to be developed for this design. Based on our experience, we have a good description for this sensor. The device has a active time period much shorted than the readout time. The active period of time is strobed by the STAR trigger, followed by a 1 ms period to read out the full number of pixels.

The general organization of the chip relies on columns processed in parallel. The chip operation includes a continuous cycling over the array with an integration time of 100 to $200 \mu \mathrm{s}$. There will be in-pixel storage of the integrated charges, but at this time it is not clear whether CDS can be done "on chip".

The details of the signal processing, i.e. ADC or double threshold discrimination for zero suppression are still open and need to be studied with real data. Studies on this chip will commence this year.

\footnotetext{
${ }^{\dagger}$ Estimate based on RHIC achieving an average $\mathrm{Au}+\mathrm{Au}$ luminosity of $1.0 \times 10^{27} \mathrm{~cm}^{-} \mathrm{s}^{-1}$ for 21 weeks at $60 \%$ efficiency. T. Roser, W. Fischer, A. Drees, H. Huang, V. Ptitsyn, "RHIC Collider Projections (FY2006-FY2008). July 19, 2005. http://www.agsrhichome.bnl.gov/AP/RHIC2004/RhicProjections.pdf
} 


\begin{tabular}{|l|l|}
\hline Ladder active area & $2 \mathrm{~cm} \times 20 \mathrm{~cm}$ \\
\hline Pixel size & $30 \mu \mathrm{m} \times 30 \mu \mathrm{m}$ \\
\hline$\sim$ Pixel mapping on the ladder & $640 \times 6400$ \\
\hline Minimum operating distance from beam & $1.5 \mathrm{~cm}$ \\
\hline Power & $\leq 200 \mathrm{~mW} / \mathrm{cm}^{2}$ \\
\hline Operating temperature & $\geq 30{ }^{\circ} \mathrm{C}$ \\
\hline Integration time & $\leq 0.2 \mathrm{~ms}$ \\
\hline Mean silicon thickness & $\leq 100 \mu \mathrm{m}$ \\
\hline Readout time & $\leq 1 \mathrm{~ms}$ \\
\hline Efficiency (min I) & $\geq 98 \%$ \\
\hline Accidental cluster density & $\leq 22 / \mathrm{cm}^{2}$ \\
\hline Binary readout, number of threshold bits & 1 or 2 \\
\hline Radiation tolerance & $\geq 171 \mathrm{kRad}$ \\
\hline Number of conductors supporting the ladder $(10 \text { chips/ladder })^{\sharp *}$ & $\leq 140$ \\
\hline Triggered readout, maximum trigger delay & $2 \mu \mathrm{s}$ \\
\hline
\end{tabular}

Table 14: Silicon requirements for maximum average Au+Au RHIC luminosity of $7.0 \times 10^{27} \mathrm{~Hz} / \mathrm{cm}^{2}$ or $2.5 \mathrm{nb}^{-1} /$ week

The time that a pixel is sensitive to tracks, this determines amount of pileup.

${ }^{\S}$ Efficiency after cluster filter on binary threshold information from the detector

${ }^{* *}$ To satisfy both the efficiency and accidental requirements it is expected that some off chip cluster analysis will be required. Depending on signal to noise either one or two thresholds will be needed.

$\Uparrow 4$ year operation at maximum RHIC luminosity for a running period of 21 weeks/year see: W. Fisher, T.

Roser, I. Ben-Zvi, A Fedotov, 16-Mar-2005

http://www.phenix.bnl.gov/phenix/WWW/publish/leitch/rhicii-forward/RHIC_II_Luminosity_Roser.pdf

$\$$ This requirement addresses the radiation thickness of the flex cable that is part of the thin ladder structure. The current proto-type ladder that uses the MIMOSA5 chips has this many conductors. At the end of the ladder there can be additional mass for cables, drivers and cooling.

$\S \S$ The short integration time allows operation of the pixel detector like any other STAR detector, namely one frame associated with one interaction event only. The STAR trigger is delivered $1.6 \mu$ following the collision. 


\section{Data Acquisition and Readout}

\subsection{Prototype and Final Design}

We are designing the readout and data acquisition system for the HFT in two stages. These stages follow the development of the silicon sensors in our project. The initial prototype readout system is designed to read out the MIMOSTAR4 detectors which have analog outputs and a $4 \mathrm{~ms}$ readout time. The second stage is for use with the final Ultimate series sensors which have a digital output and a $1 \mathrm{~ms}$ readout time. The goal of the first stage is to develop much of the infrastructure for doing cluster finding and data sparsification, the interfaces to trigger and DAQ and the mechanical readout structures and assemble a working prototype detector with the MIMOSTAR4 sensors. The second stage would make use of the development that we have done with the prototype detector and integrate the Ultimate series sensors with a developed readout system.

\subsection{Requirements and Prototype Design}

The requirements for the prototype and final readout system are very similar. They include:

- Triggered detector system fitting into existing STAR infrastructure and interfaces to the existing Trigger and DAQ systems.

- Deliver full frame events to STAR DAQ for event building at approximately the same rate as the TPC.

- Reduce the total data rate of the detector to a manageable level ( $<$ TPC rate)

We have designed the prototype data acquisition system to read out the large body of data from the individual MIMOSA4 sensors, to digitize the signals, to perform data compression, and to deliver the sparsified data to an event building and storage device. A summary of the specifications and requirements is provided in Table 15.

\begin{tabular}{|l|l|}
\hline Total number of pixels & $98 \times 10^{6}$ \\
\hline Number of pixels per chip & $640 \times 640$ \\
\hline Pixel Readout rate (analog output) & $2 \times 50 \mathrm{MHz} /$ chip \\
\hline Readout time per frame & $4 \mathrm{~ms}$ \\
\hline Frame integration time & $4 \mathrm{~ms}$ \\
\hline Fixed pattern noise & $2000 e^{-}$ \\
\hline Noise after Correlated Double Sampling & $10 e^{-}$ \\
\hline Maximum signal & $900 e^{-}$ \\
\hline Dynamic range after Correlated Double Sampling & $8 \mathrm{bits}$ \\
\hline Total power consumption (24 ladders) & $90 \mathrm{~W}$ \\
\hline
\end{tabular}

Table 15: Prototype Stage Requirement Summary - constraints for the MIMOSTAR4 APS. 
Digitizing the analog signal on each pixel into a 10 bit digital signal yields approximately $1 \mathrm{~Gb} / \mathrm{s}$ per sensor chip when read out in $4 \mathrm{~ms}$. Thus, the total front end data rate is $\sim 240$ $\mathrm{Gb} / \mathrm{s}$. Clearly, the volume of data must be reduced before being passed to the DAQ event builder and written to storage.

Data compression is achieved by performing correlated double sampling (CDS) and then leakage current subtraction, i.e. subtraction of two consecutive frames followed by zero suppression. CDS cancels out fix pattern and reset noise and reduces $1 / \mathrm{f}$ noise. The fixed pattern noise corresponds to the spread of the baseline voltage in all pixels. It has been measured on the MIMOSA- 5 chip to be 2000 electrons. The noise remaining after CDS must be on the order of $14 e^{-}$to guarantee an efficiency of greater than $98 \%$. The maximum signal is estimated from $\mathrm{dE} / \mathrm{dx}$ calculation and by measuring how the charge spreads over pixels. The signal can be truncated above $900 e^{-}$without compromising either the efficiency or the position resolution, so 8 bits is a sufficient dynamic range for signal storage. A synchronous cluster finding algorithm and the reduction of the data to addresses of cluster center pixels reduce the data to a manageable rate.

\subsection{Architecture for the Prototype System}

The basic flow of a ladder data path starts with the APS sensors. An HFT ladder has 10 MIMOSTAR4 APS chips each with a 640 by 640 pixel array. Each chip is divided in half with two sectors each containing a separate analogue, differential current output buffer. The chips are continuously clocked at $50 \mathrm{MHz}$ and the data is read out, running serially through all the pixels connecting them to the output buffer. This operation is continuous during the operation of the MIMOSTAR detectors on the HFT ladder. Analog data is carried from the two $50 \mathrm{MHz}$ outputs in each sensor in parallel on a low mass ladder flex printed circuit board to discrete electronics at the end of the ladder and out of the low mass detector region. This electronics performs current to voltage conversion and contains buffers and drivers for the clocks and other control signals needed for ladder operation.

Each MIMOSTAR detector requires a JTAG connection for configuration of the chip, power, ground and a $50 \mathrm{MHz}$ readout clock. These signals and power as well as the analog outputs from the detectors are carried via a low mass twisted pair cable from the discrete electronics at the end of the ladder to the readout electronics located about 1 meter from the HFT ladders. Each ladder and associated readout board are the same and there is one readout board per HFT ladder. A functional diagram of an HFT ladder and a description of the data flow are shown in Figure 38. 


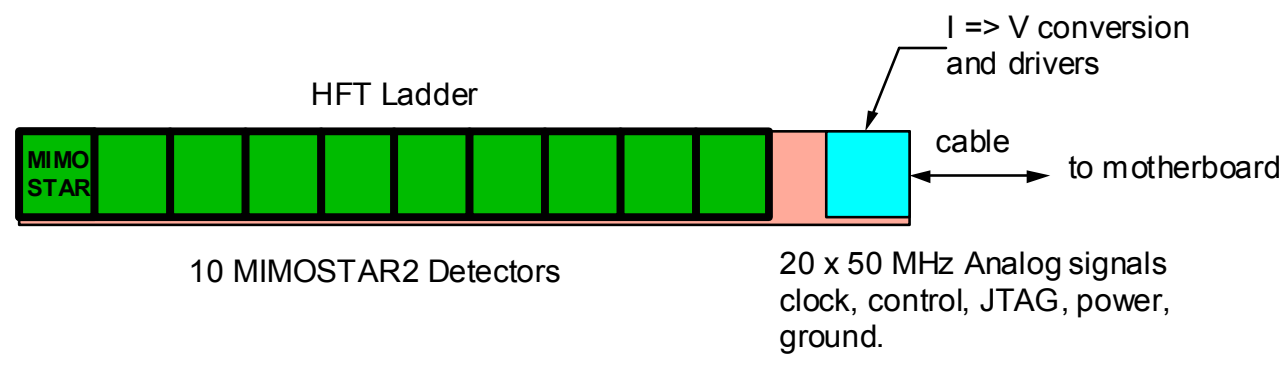

Figure 38: Ladder Layout - sketch of the readout-topology on a detector ladder. This figure shows the ten APS and the corresponding current to voltage conversion and driver electronics. The drivers will be located out of the low mass region of the detector and may require additional cooling.

The readout electronics consist of a motherboard and daughter card configuration. A functional block diagram is shown in Figure 39. There are 5 daughter cards per motherboard and each daughter card services 2 of the MIMOSTAR sensors on the ladder. The analog signals are carried to the daughter cards where they are digitized with a 10-bit $\mathrm{ADC}$ at $50 \mathrm{MHz}$. Following digitization, the 10-bit ADC values are passed synchronously to an FPGA for CDS. Performing CDS and pedestal subtraction requires a data sample to be stored for each pixel of the detector. This drives the need for external RAM on the daughter cards. After CDS and pedestal subtraction, 8 bits can represent the data. The data is then transferred to the next stage for hit finding and data reduction. 


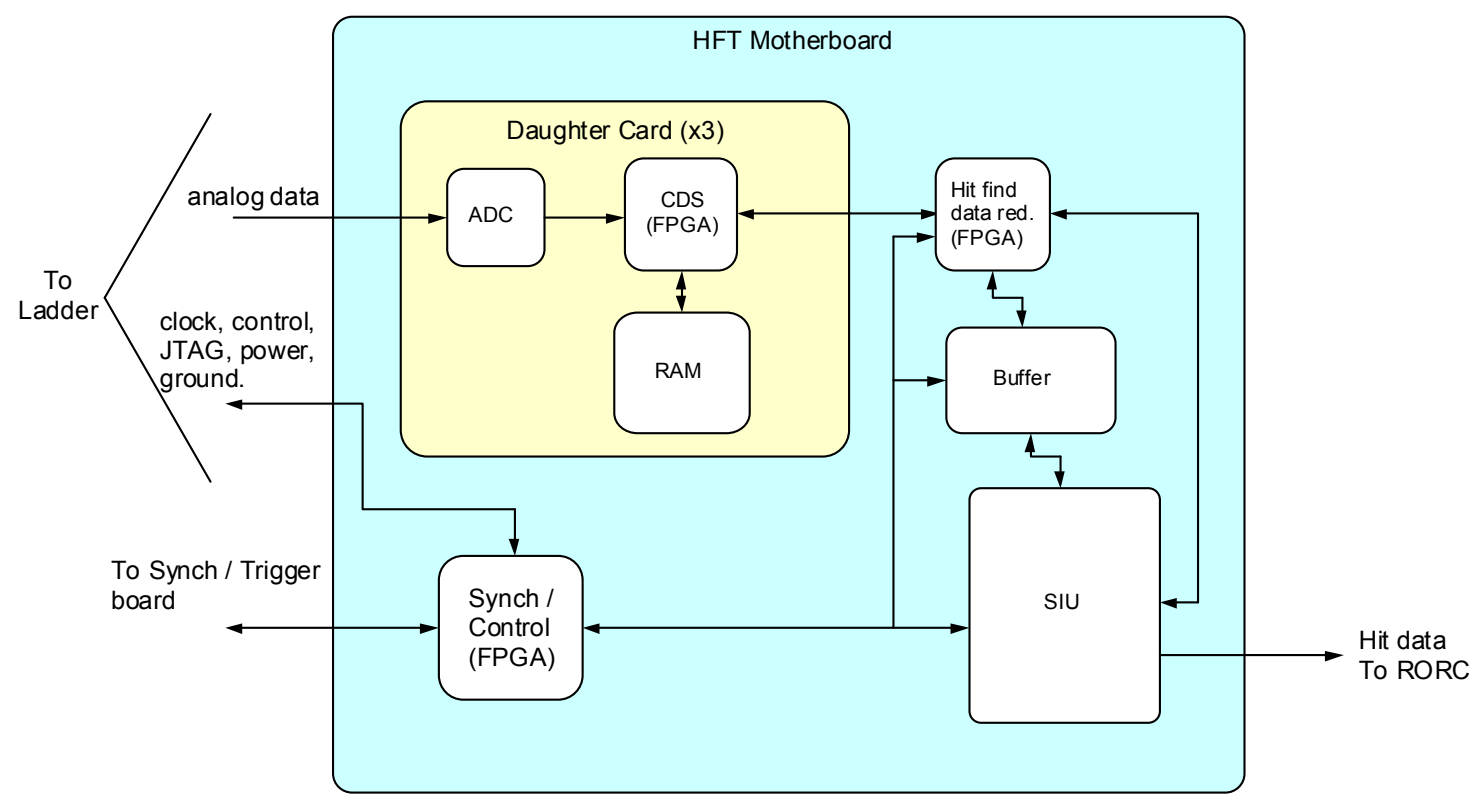

Figure 39: Prototype DAQ Layout: schematic of DAQ system for a single MIMOSTAR4 ladder. Analog data is carried as differential current on the low mass cable at $50 \mathrm{MHz}$. The signals are driven in parallel over short $(\sim 1 \mathrm{~m})$ twisted pair cables to the motherboard. Analog to digital conversion, CDS and data reduction are performed in the Motherboard / Daughter cards. The reduced hit data is transferred digitally to the SIU and carried to Linux based readout PCs via an optical fiber. Control, synchronization, and event ID tagging are accomplished in the Synch/Trigger FPGA on the motherboard.

The 8-bit data data exiting the CDS stage is resorted on the fly to be a traditional raster scan through the pixels of the sector. This stream of rasterized data can then be passed to the cluster finder. We are currently investigating methods of hit finding and data reduction for use on the motherboard. A simple readout of the address of a center pixel high threshold hit with the surrounding 8 pixels meeting additional cluster selection criteria such as at least 1 cell over the low threshold is our default approach. This can be implemented in an FPGA and run as a pipeline filling the output buffer with center pixel address values. A simple example of an FPGA logic diagram that accomplishes this can be found in.Figure 40 . We are also investigating a number of cluster selection methods including summing algorithms around different thresholds and center pixel determination by geometric pattern with high and low thresholds. A preliminary study of some simple and FPGA implementable cluster finding algorithms shows promising results for efficiency and noise rejection. A sample of these results can be seen in Figure 41. An implementation document using this method of hit finding saving only the center pixel address of the cluster is available as an appendix. 


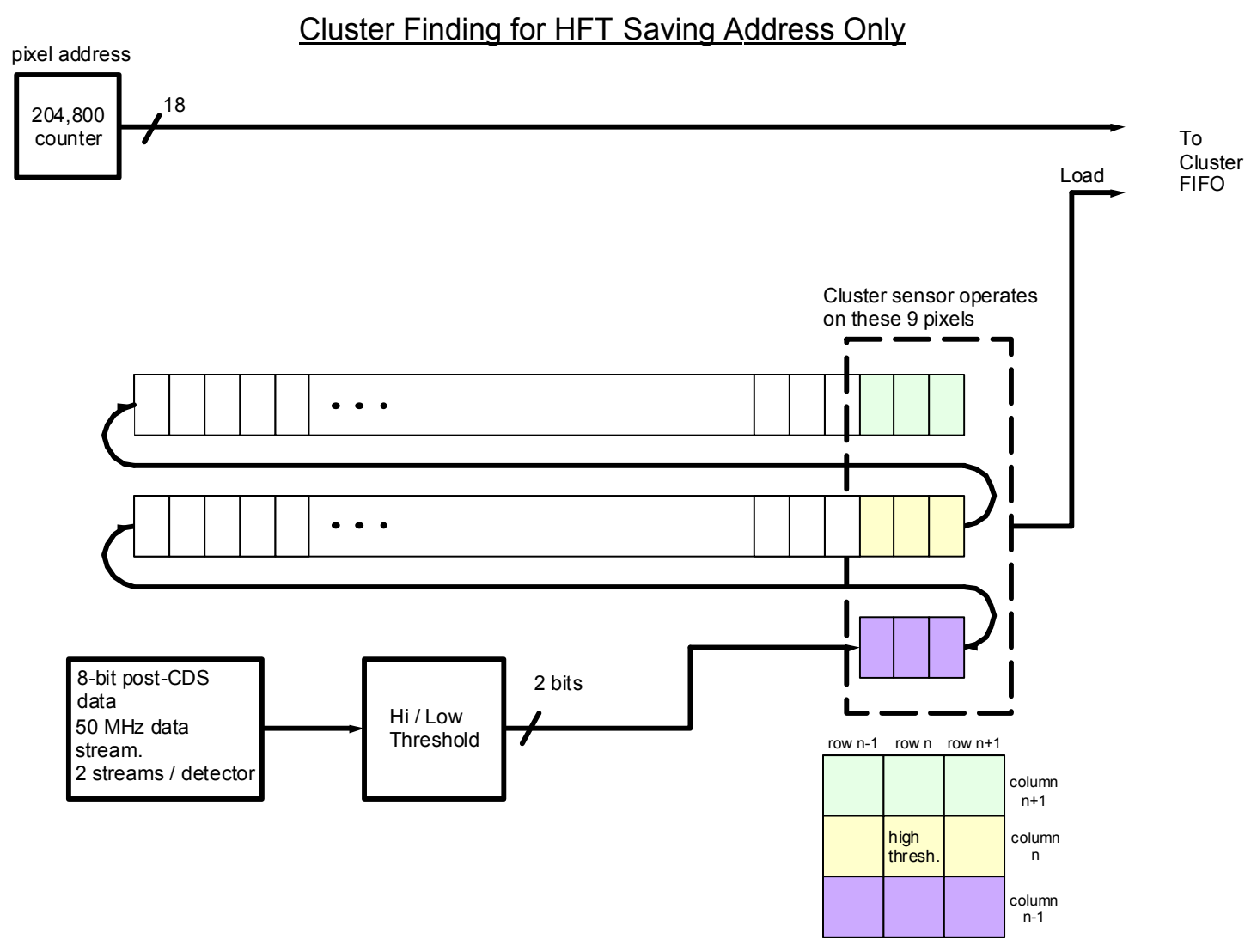

Figure 40: A simple cluster finding algorithm for the HFT detector. ADC data from two MIMOSTAR detector columns +3 pixels are sent to a high/low threshold discriminator. The resulting 2 bits are fed sequentially in an 2-bit wide shift register. The center pixel of a $3 \times 3$ pixel window is compared to a high threshold with each clock tick. If the threshold is exceeded, the additional cluster identification criteria are checked for the $3 \times 3$ pixel window. If the results meet the critera for a cluster, the center pixel address is stored into a readout FIFO. This method is extendable to allow for multiple simultaneous thresholds and geometric pattern triggers. 


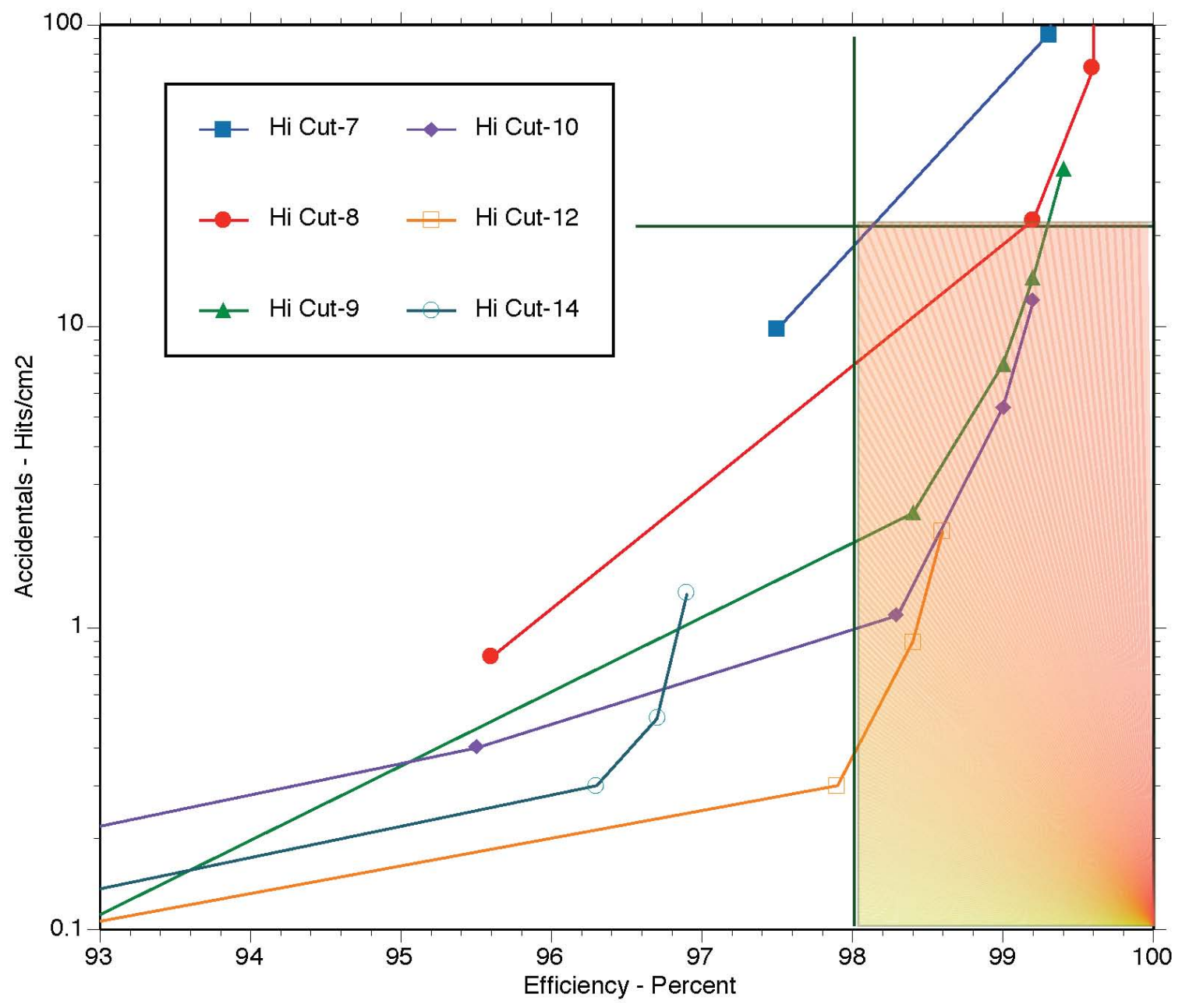

Figure 41: Efficiency versus accidentals for a cluster finding algorithms run on cluster data from a MIMOSA5 detector. Note that some parameter combinations of this algorithm are already over $98 \%$ efficient with a accidentals rate of $1-2$ hits $/ \mathrm{cm}^{2}$.

The reduced data is then buffered and transferred to the STAR DAQ system over a highspeed bi-directional fiber link. We intend to use the Source Interface Unit (SIU) and Readout Receiver Cards (RORC) developed for ALICE as our optical link hardware to transfer data to and from the STAR DAQ system. These links have been chosen as the primary readout connections for the new STAR TPC FEE. Leveraging existing hardware and expertise in STAR allows for a faster and more reliable design than developing our own custom solution. The complete system consists of a parallel set of ladder readouts consisting of 24 separate chains.

\subsection{Data Synchronization, Readout and Latency}

The readout of the prototype HFT sensors is continuous and hit and cluster finding is always in operation during the normal running of the detector. The receipt of a trigger initiates the saving of the found clusters into a FIFO for 1 frame (204,800 pixels). The 
HFT detector as a whole will be triggered via the standard STAR TCD module. Since 4 $\mathrm{ms}$ are required to read out the complete frame of interest, the data will be passed to DAQ for event building $\sim 4 \mathrm{~ms}$ after the trigger is received. In order to service multiple triggers within that $4 \mathrm{~ms}$ readout time we will provide multiple buffers that will allow the capture of temporally overlapping complete frames. A functional block diagram of this system is shown in Figure 42. In this system, the cluster data is fanned out to 5 Event FIFOs. A separate Event FIFO is enabled for the duration of one frame upon the receipt of a trigger from the TCD. Subsequent triggers enable additional Event FIFOs until all of the event FIFOs are full and the system goes busy. The resulting separate complete frames are then passed to STAR DAQ as they are completed in the Event FIFOs. This multiple stream buffering gives a system that can be triggered up to the expected rate of the STAR TPC (approximately $1 \mathrm{KHz}$ ) after the DAQ1K upgrade. This will result in the duplication of some data in frames that overlap in time, but our data rate is low and the duplication of some data allows for contiguous event building in the STAR DAQ, which greatly eases the offline analysis. In addition, synchronization between the ladders/boards must be maintained. The HFT will receive a clock via the standard STAR TCD and will derive its internal clocks from the RHIC strobe. We will provide functionality to allow the motherboards to be synchronized at startup and any point thereafter.

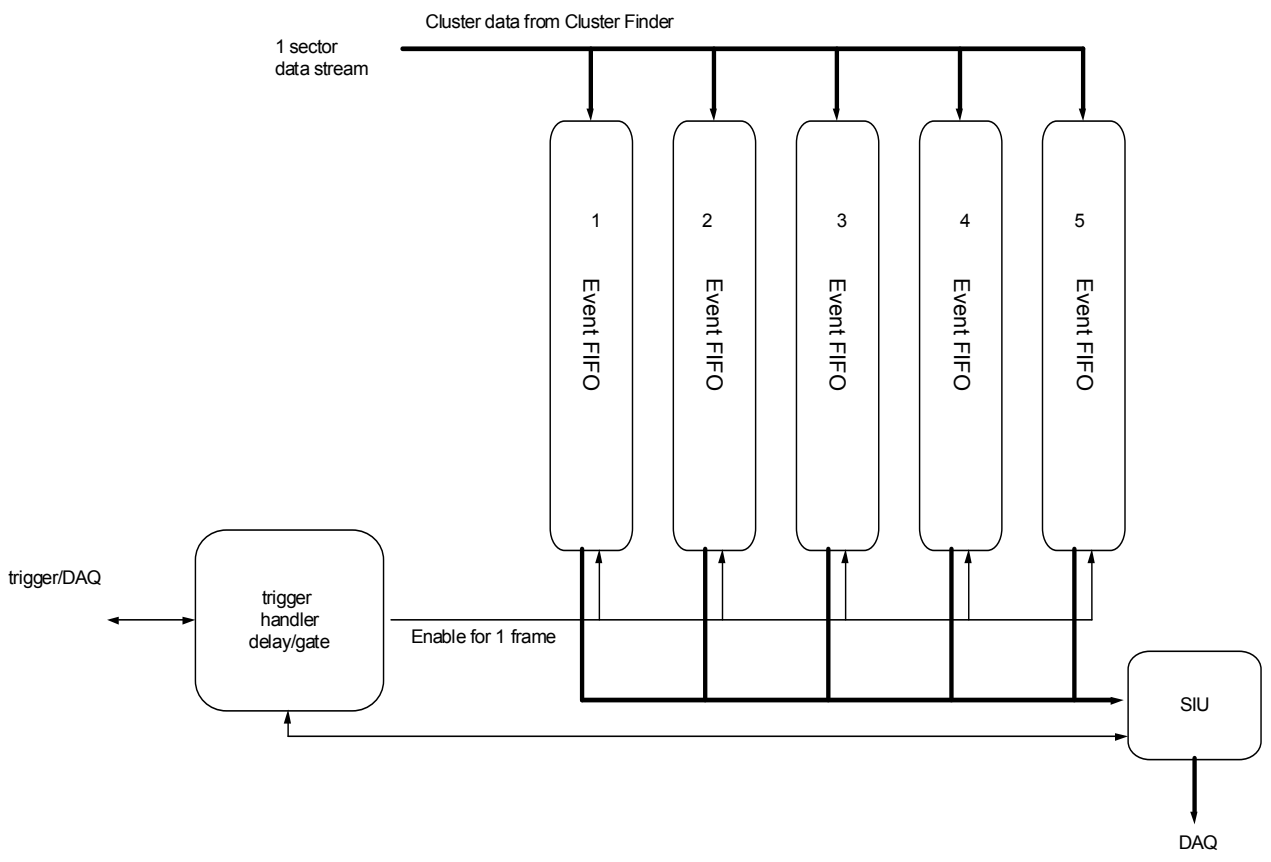

Figure 42: Multiple event FIFOs are fed in parallel from the cluster finder. A separate Event FIFO is enabled for one frame upon the receipt of a trigger from the TCD. The resulting separate complete frames are then passed to STAR DAQ as they are completed in the Event FIFOs. 


\subsection{Data Rates for the Prototype RDO}

\begin{tabular}{|l|c|}
\hline$\underline{\text { Item }}$ & $\underline{\text { Number }}$ \\
\hline bits/address & 18 \\
\hline inner ladders & 6 \\
\hline outer ladders & 18 \\
\hline MIMOSTAR sectors per ladder & 20 \\
\hline average hits/sector, inner, $\mathrm{L}=10^{27}$ & 245 \\
\hline average hits/sector, outer, $\mathrm{L}=10^{27}$ & 49 \\
\hline
\end{tabular}

Table 16: Data rate calculation parameters

The data rate from each $640 \times 640$ MIMOSTAR detector is thus approximately $1 \mathrm{~Gb} /$ sec. The total rate of raw data entering the processing chain in the detector is thus approximately $240 \mathrm{~Gb} / \mathrm{sec}$. After CDS, the data can be represented by 8 bits. Pixel addressing within a sector requires 18 bits. The sector-in-ladder address will be accomplished as address words in the data stream. Ladder address will be added at the DAQ receivers. This covers the address space to map the detector pixel space. Each cluster word stored in the FIFO contains the 18 address bits of a cluster central pixel. Combining this with the occupancy per layer and the readout rate of $1 \mathrm{KHz}$ gives an event size of $106 \mathrm{~KB}$ and data rate from the detector of $106 \mathrm{Mb} / \mathrm{sec}$. Figure 43 shows this graphically.

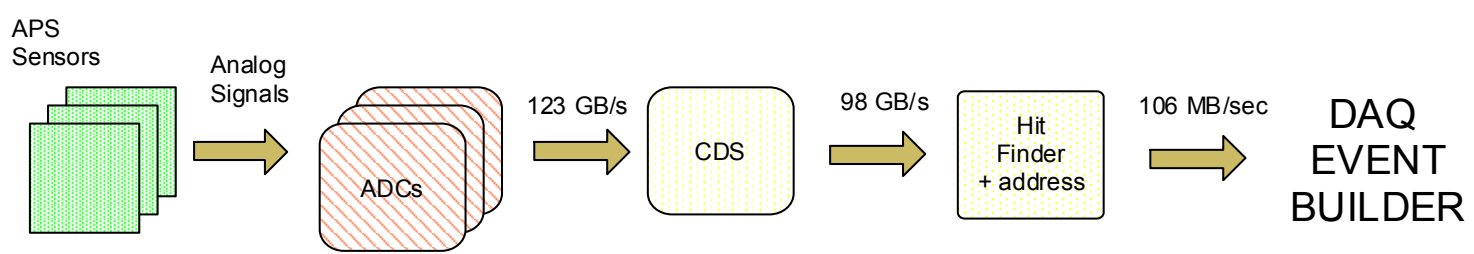

Figure 43: Data rates at the various stages of the Prototype MIMOSTAR4 readout chain.

\subsection{Requirements for the Ultimate Design}

The Ultimate series of APS detector will incorporate several changes from the previous MIMOSTAR versions. The primary changes include on pixel CDS and a two level programmable discriminator applied to the CDS output for each chip. The Ultimate chip will be read out digitally in 2 bit words / pixel through 4 LVDS outputs / chip. The control functions for the chip are still via the JTAG interface. A summary of the new 
specifications is provided in Table 17. The basic requirements are as indicated previously.

\begin{tabular}{|l|l|}
\hline Total number of pixels & $98 \times 10^{6}$ \\
\hline Number of pixels per chip & $640 \times 640$ \\
\hline Pixel Readout rate & $\begin{array}{l}4 \times 250 \mathrm{Mb} / \mathrm{s} \\
\text { LVDS / chip }\end{array}$ \\
\hline Readout time per frame & $1 \mathrm{~ms}$ \\
\hline Frame integration time & $200 \mu \mathrm{s}$ \\
\hline $\begin{array}{l}\text { Internal configurable Discriminators } \\
\text { (post internal CDS) }\end{array}$ & $2 \mathrm{bits}$ \\
\hline Raw data from one sensor & $820 \mathrm{Mb} / \mathrm{s}$ \\
\hline Total power consumption & $90 \mathrm{~W}$ \\
\hline
\end{tabular}

Table 17: Final Stage Specification Summary - constraints for the ULTRA series APS.

This system readout is a bit different than the previous MIMOSTAR4 based readout but most components are the same.

\subsection{Architecture for the Ultimate System}

In this system, the much of the functionality of the daughter cards has been moved into the Ultimate sensors themselves. The correlated double sampling and dual level discriminator functionality are now integrated onto the sensor and there are 4 LVDS readout lines / chip. The rest of the system remains substantially the same however. A revised functional block diagram is shown below in Figure 44.

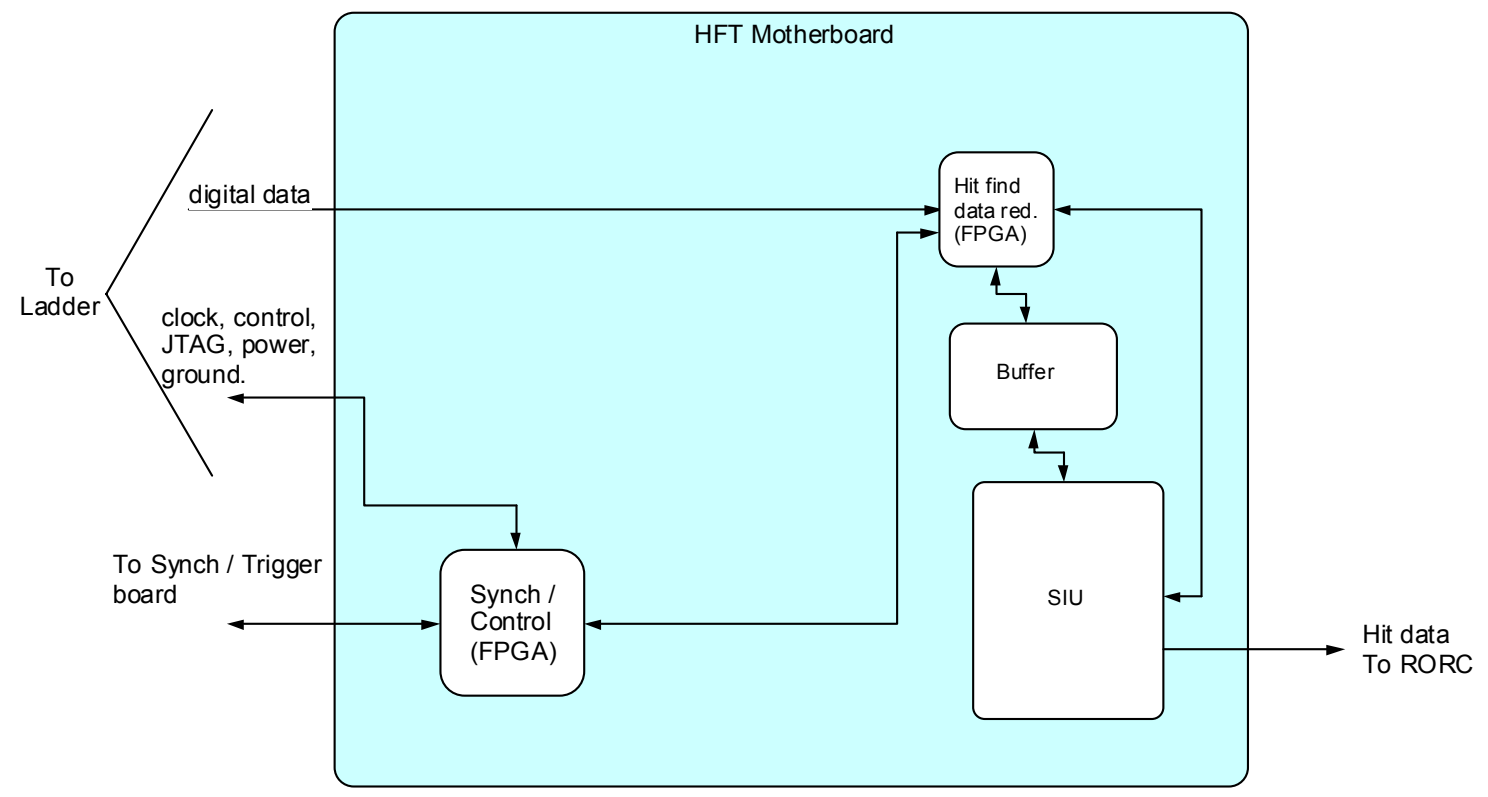

Figure 44: Functional block diagram for Ultimate sensor based readout system. 
In this system digital 2 bit words representing high / low discriminator threshold crossings are fed into the cluster finding FPGAs via 4 LVDS lines per sensor. The data will still be delivered in a sorted raster scan so our cluster finding algorithms will process the data in the same way as before. The cluster center addresses are again passed into the event FIFOs for readout to DAQ. There is a difference in the way triggers are processed. At the current level of design, this will be a triggered system that goes dead for the $1 \mathrm{~ms}$ readout time required to move the data to and through the cluster finding and into the event FIFOs. This is a change in that while we will employ multiple event buffering, the system will not run continuously with data always being processed and event FIFOS filling simultaneously, event FIFOs will be filled sequentially as triggers are received and read out by DAQ as they are filled. The event rate requirement of $\sim 1 \mathrm{KHz}$ is still met by this modified design.

\subsection{Data Rates for the Ultimate RDO}

\begin{tabular}{|l|l|}
\hline$\underline{\text { Item }}$ & Number \\
\hline bits/address & 18 \\
\hline inner ladders & 6 \\
\hline outer ladders & 18 \\
\hline Ultimate sectors per ladder & 40 \\
\hline average hits/sector, inner, $\mathrm{L}=10^{27}$ & 6.1 \\
\hline average hits/sector, outer, $\mathrm{L}=10^{27}$ & 1.2 \\
\hline
\end{tabular}

Table 18: Data rate calculation parameters

The data rate from each $640 \times 640$ Ultimate detector is $102 \mathrm{MB} / \mathrm{sec}$. The total rate of raw data entering the processing chain in the detector is thus approximately $2.45 \mathrm{~Gb} / \mathrm{sec}$. Pixel addressing within a sector still requires 18 bits. Combining this with the occupancy per layer and the readout rate of $1 \mathrm{KHz}$ gives an event size of $5.24 \mathrm{~KB}$ and data rate from the detector of $5.24 \mathrm{Mb} / \mathrm{sec}$. Figure 45 shows this graphically.
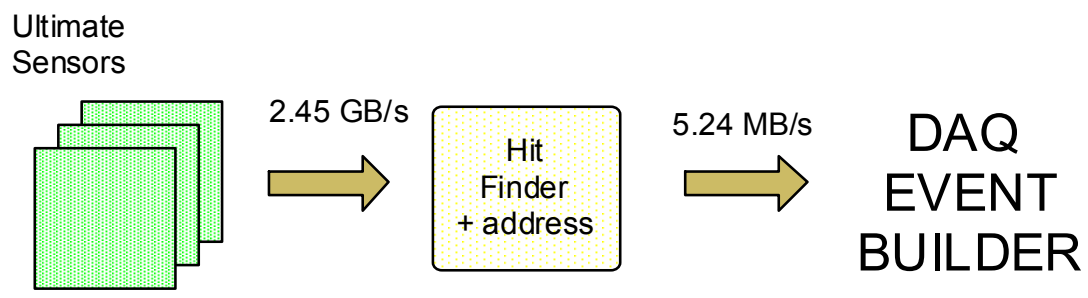

Figure 45: Data rates in ULTIMATE HFT readout. 


\subsection{Prototypes}

Several different prototype readout electronics boards have been constructed and tested that are very similar to the proposed prototype readout electronics described above. In Figure 46 one can see the prototyping results of a low mass flex PCB on a prototype ladder with MIMOSA5 detectors.

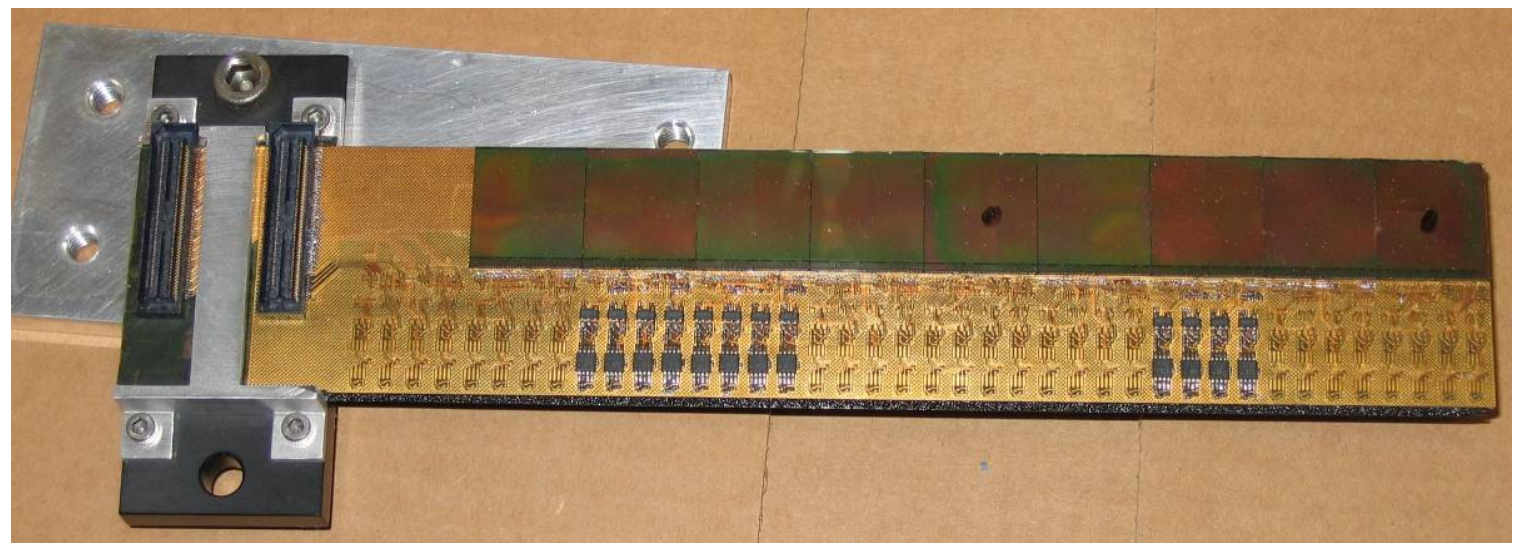

Figure 46: A prototype ladder showing low mass PCB, MIMOSA5 detectors and driver electronics bonded to a mechanical carbon fiber and reticulated vitreous carbon foam based carrier.

A prototype readout system for reading the MIMOSA5 detectors was also constructed and used. A functional schematic is shown below in Figure 47. 


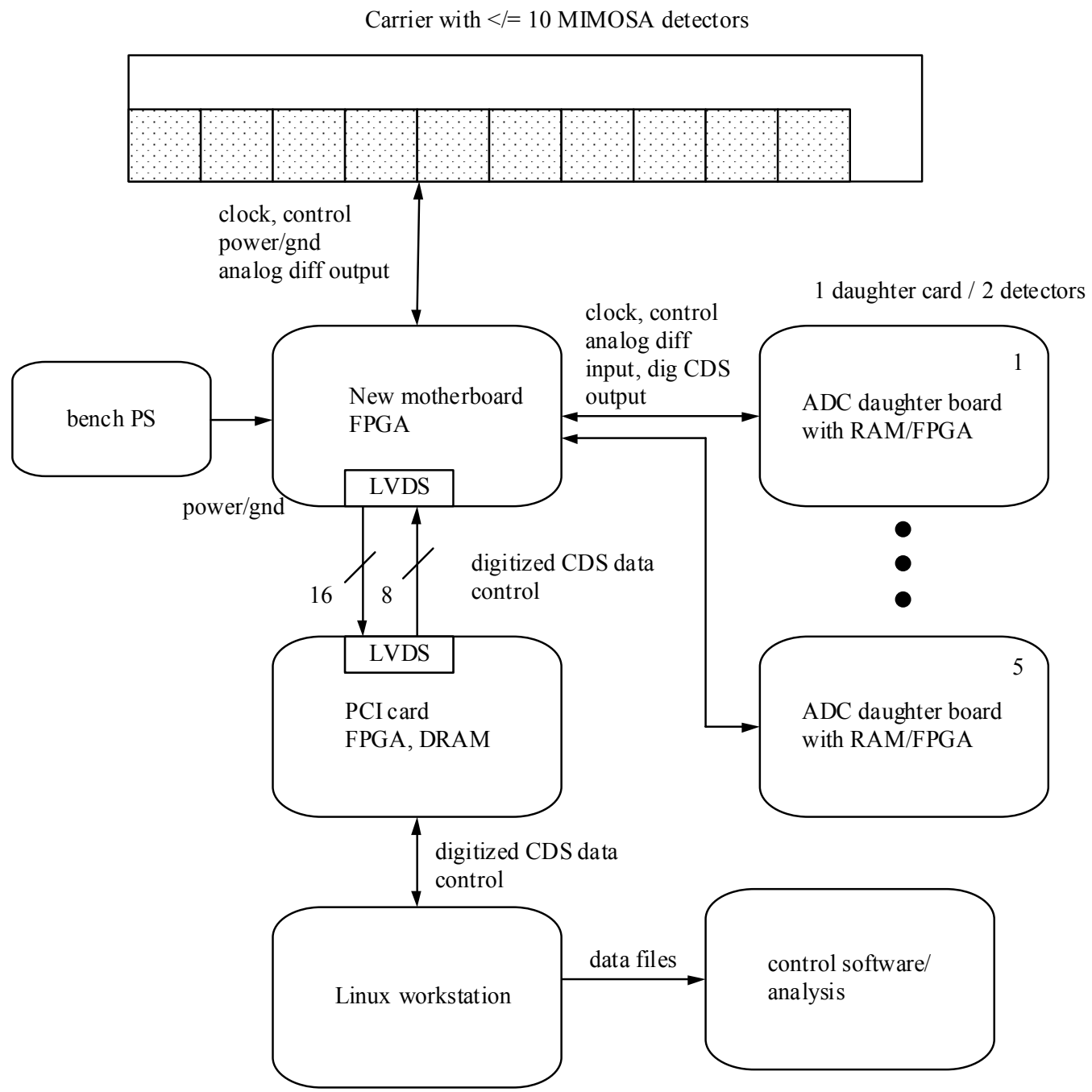

Figure 47: Functional component diagram of the prototype readout system constructed for the readout on a MIMOSA5 based ladder.

One can see the great similarity between what we have constructed for this test and the prototype MIMOSTAR4 readout design. The basic data flow is the same. The details of hit finding and final readout to DAQ are not present in this system but the motherboard / daughter card concept and the ADC FPGA and SDRAM for CDS are present. A photograph showing the motherboard with a daughter card attached is shown in Figure 48. 


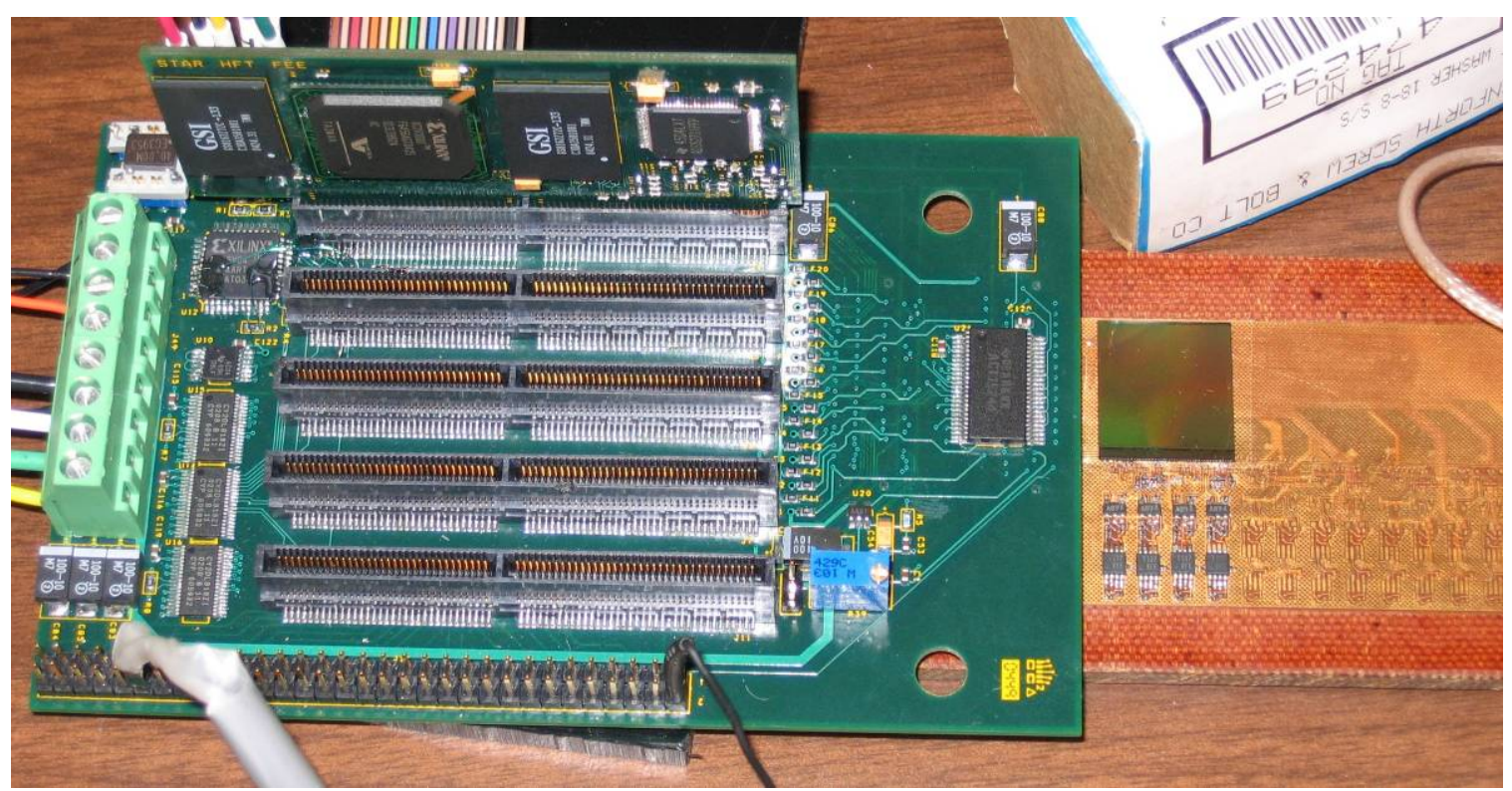

Figure 48: Early Prototype motherboard and daughter card used for reading out MIMOSA5 detectors.

This system is working and the basic elements of FPGA based control and deserialization of the ADC outputs, SDRAM memory interface and CDS are implemented on the daughter cards seen above. We will use these VHDL elements in our final design. The daughter card design above is quite flexible and the same physical boards may be suitable for the prototype HFT readout system. 


\section{Integration with STAR}

\subsection{Mechanical Introduction}

Simulations have shown that for good D meson identification in the high track density environment of $\mathrm{Au}+\mathrm{Au}$ collisions, it is important to minimize the scattering thickness of the beam pipe and the first detection layer. This requirement has been the primary driver of the current design. By using APS technology we can use thinned silicon with modest connection requirements to minimize the support electronics and cable thickness. In addition, the low power nature of these devices allows a mechanical design that is aircooled which helps to minimize scattering material in the track path. Several conceptual designs have been considered. We present the design that is currently the focus of our investigations.

In recognition of difficulties encountered in a variety of experiments, we are adopting design requirements for rapid insertion and removal of the vertex detector, rapid calibration and calibration transfer and multiple detector copies. By addressing these issues early in the design cycle the requirements can be met without major cost impact.

Our mechanical design makes significant use of carbon composite material, which has nearly the same radiation length as beryllium. This allows us to take advantage of the extensive work going on at LBNL for the ATLAS pixel detector. This will be a significant advantage when we move into the production phase of the project.

Since the design of the original STAR detector system, there has been significant progress in tools available for mechanical work. Very powerful low cost 3D CAD programs are now available which allow complex modeling with moving parts and direct interfacing to $\mathrm{CNC}$ machines and rapid prototyping. These tools provide the means to tackle the more complex mechanical designs required for rapid insertion and alignment. The tools are much easier to learn than older software, but because of their newness, there is still a limited base of experienced users to talk to. The R\&D effort to develop fabrication and fixturing methods is allowing us to gain the required expertise with these new tools.

\subsection{Support Carriage for Rapid Installation and Removal}

We have started to evaluate our design with test structures, detector carrier support elements, and digitizing and readout electronics boards.

A conceptual design has been developed for the support carriage that will permit rapid insertion and removal without moving other detector components. This system is shown in Figure 49 as it will be positioned for operation in the STAR detector system. The HFT 
is a small detector at the center of the STAR system. An additional layer of tracking (not shown), the SSD, lies out side of the HFT. Room is left for another detector between the SSD and the HFT that can be added at a later date for improved performance. As shown, the mechanical support and electronic service for the HFT is located on one end only. The light weight guide track and support bridge are permanent elements of the HFT design. The detector system permits shown in the figure allows for rapid removal and replacement while maintaining reproducible position through the use of fully defined kinematic mounts. For simplicity several existing STAR structures such as the SSD and one of the cone supports that is integral to its support are not shown

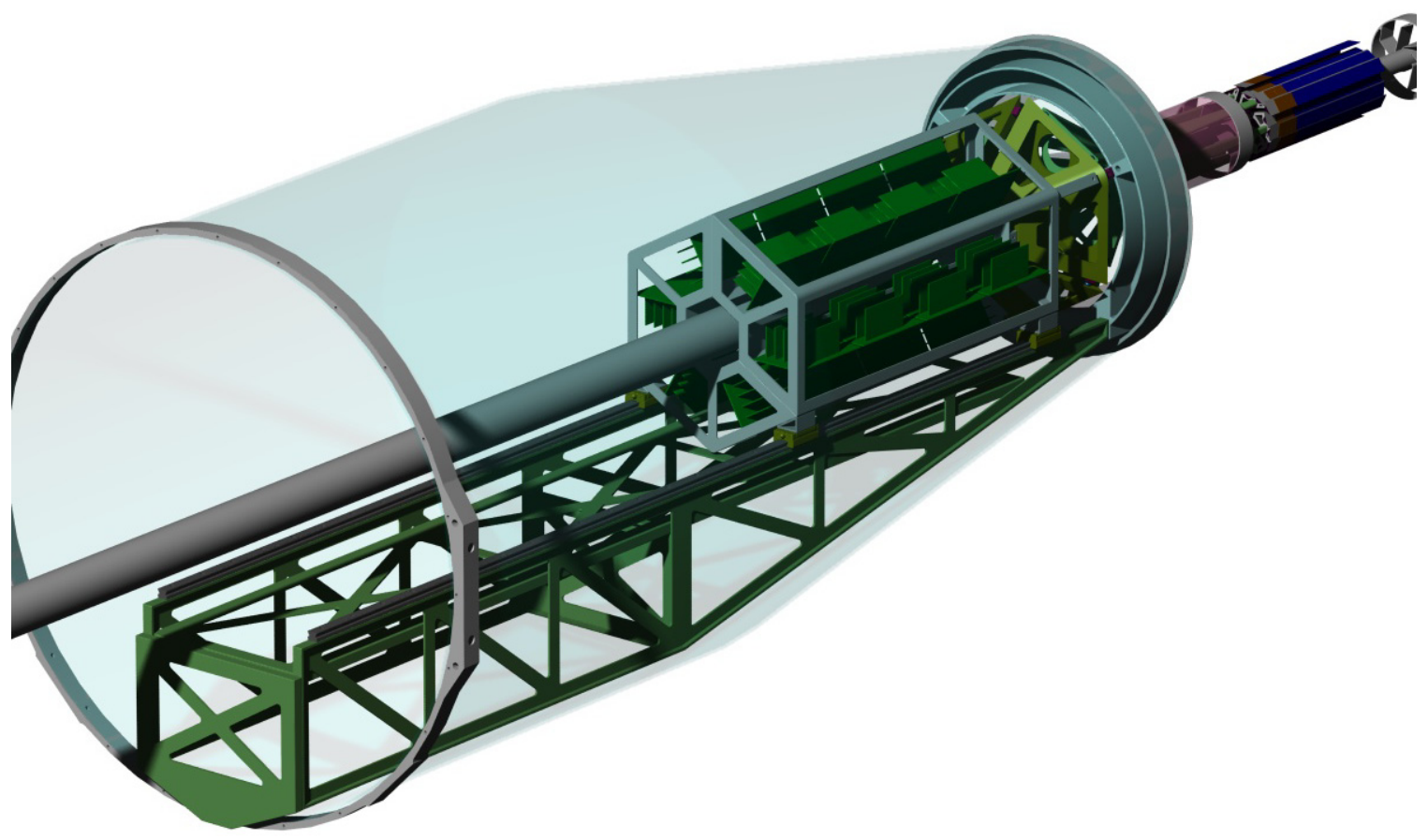

Figure 49: The HFT is shown integrated with the STAR inner detectors cone assembly.

A close up view in Figure 50 shows the HFT ladder arrangement. There are two tracking layers: one at $1.5 \mathrm{~cm}$ radius and the other at $5.0 \mathrm{~cm}$ radius. The ladder design shown in the figure consists of a single thin gull shaped carbon fiber beam with a flex PC cable plus multiple thinned silicon APS detector chips. Other two layer carbon fiber laminates are being developed which are simpler to construct but pose additional challenges in terms of mechanical integration. 


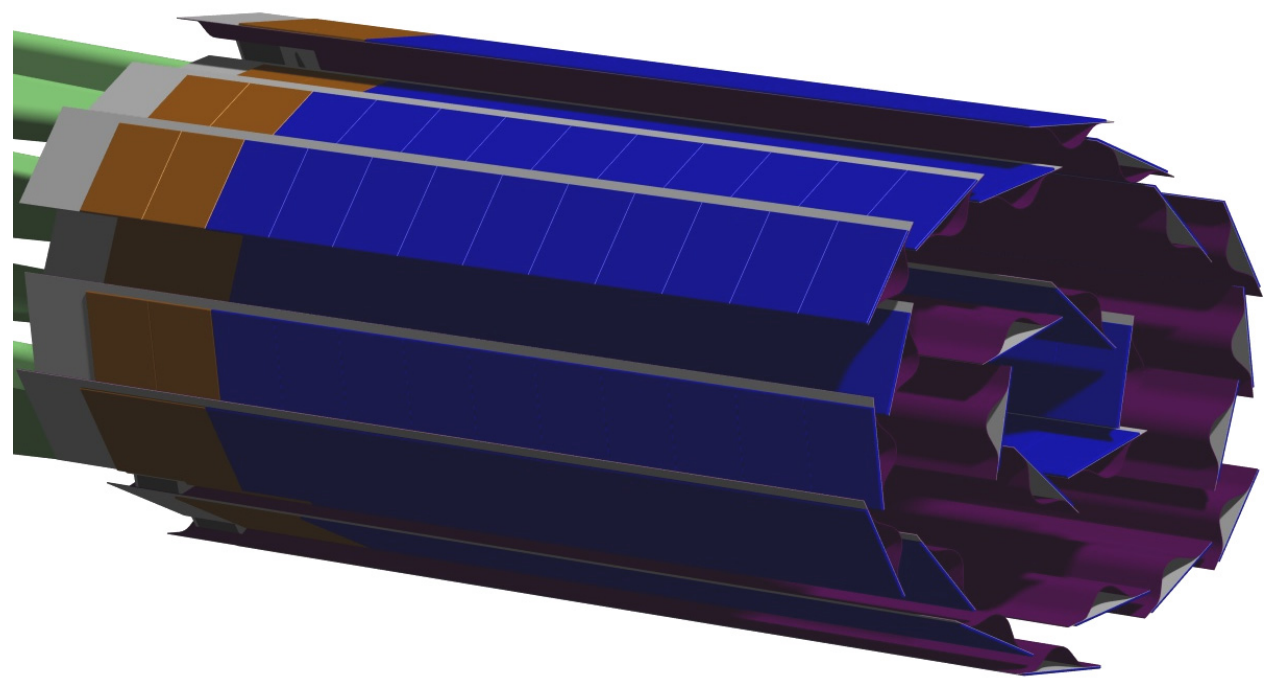

Figure 50: Close up view of the HFT ladders.

The detector support structure illustrated in Figure 51 shows elements of the kinematic position control assembly. The detector ladders are arranged in 6 modules with 3 outer layer ladders and 1 inner ladder on each module. The module is supported on a long structure, shown in green. The long green structure allows stable support of the ladder while avoiding contact with cabling and other structures that are part of the intermediate tracking system The brass colored structures provide common tie points for coupling the detector system in a reproducible manner. A more detailed view of this support system appears in Figure 52 showing the three identical frames (brass colored) that are kinematically joined around the beam pipe using ball on cylinder contacts.

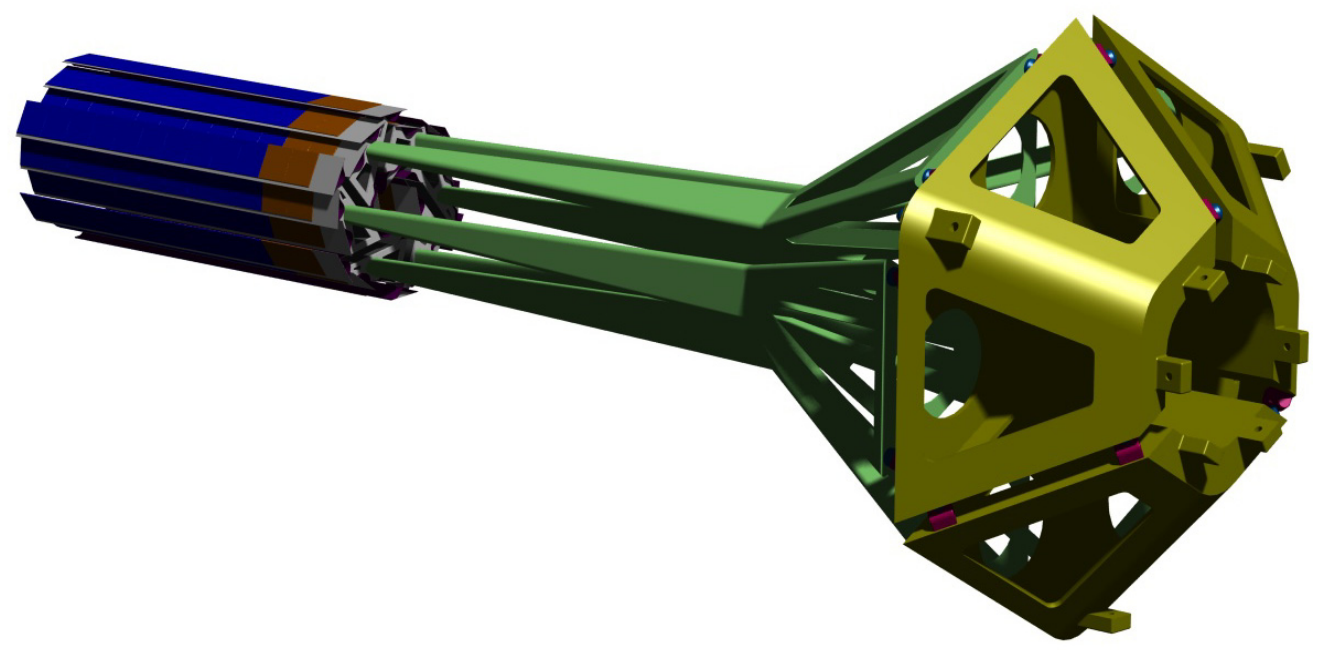

Figure 51: Detector support structure with kinematic mounts to insure repeatable detector positioning. 
The system provides three point kinematic mounts for each of the detector module stalks. This allows opening of the ladders to pass over the large radius region of the beam pipe during detector insertion and removal. The three multi coupling structures are joined with each other (a total of 6 contact points joining the three structures) again providing a defined reproducible assembly in lockdown while allowing separation for disassembly from around the beam pipe. Finally there is an additional 3 point kinematic mount on the 3 fold multi coupler to lock it to the STAR support cone. All the contacts use commercially available balls on cylinder pairs. Releasable capture mechanisms are required to load the kinematic mounts. These devices are not shown in the illustrations.

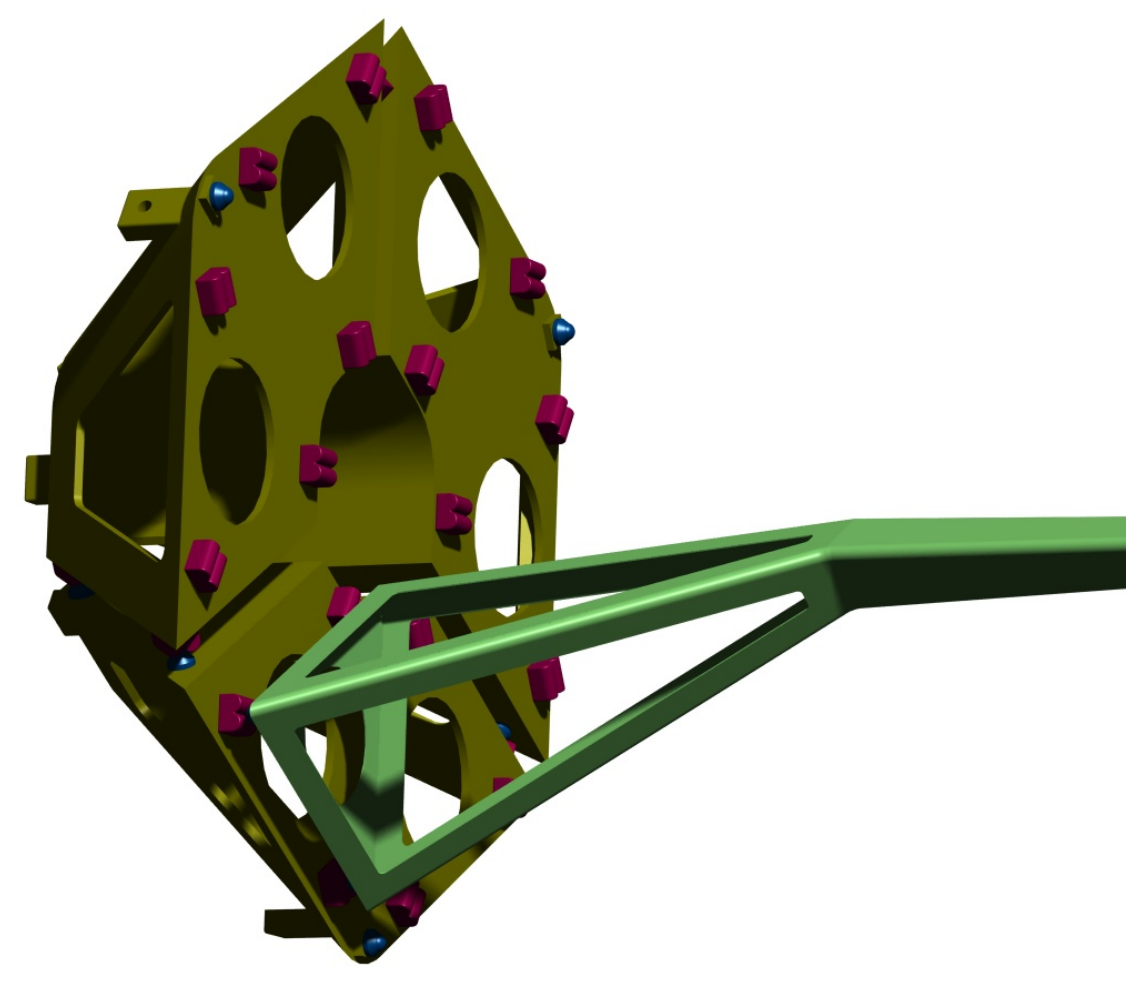

Figure 52: Kinematic mount structure.

The common tie point joining the HFT detector system to the STAR support cone is illustrated in Figure 53. The blue-grey piece is the final common tie point for critical mating parts. It supports the beam pipe wheel structure, the kinematic detector frame, the roll-in rails and joins the HFT detector assembly as a whole to the existing STAR support cone. This design will allow preassembly and testing of the critical matching pieces prior to final installation into the STAR system. The final installation requires simply securing the blue-grey piece to the STAR support cone using existing bolt holes. Note, the card barrel is loosely coupled to the detector cluster giving freedom for the kinematic mount to define final precision positioning. 


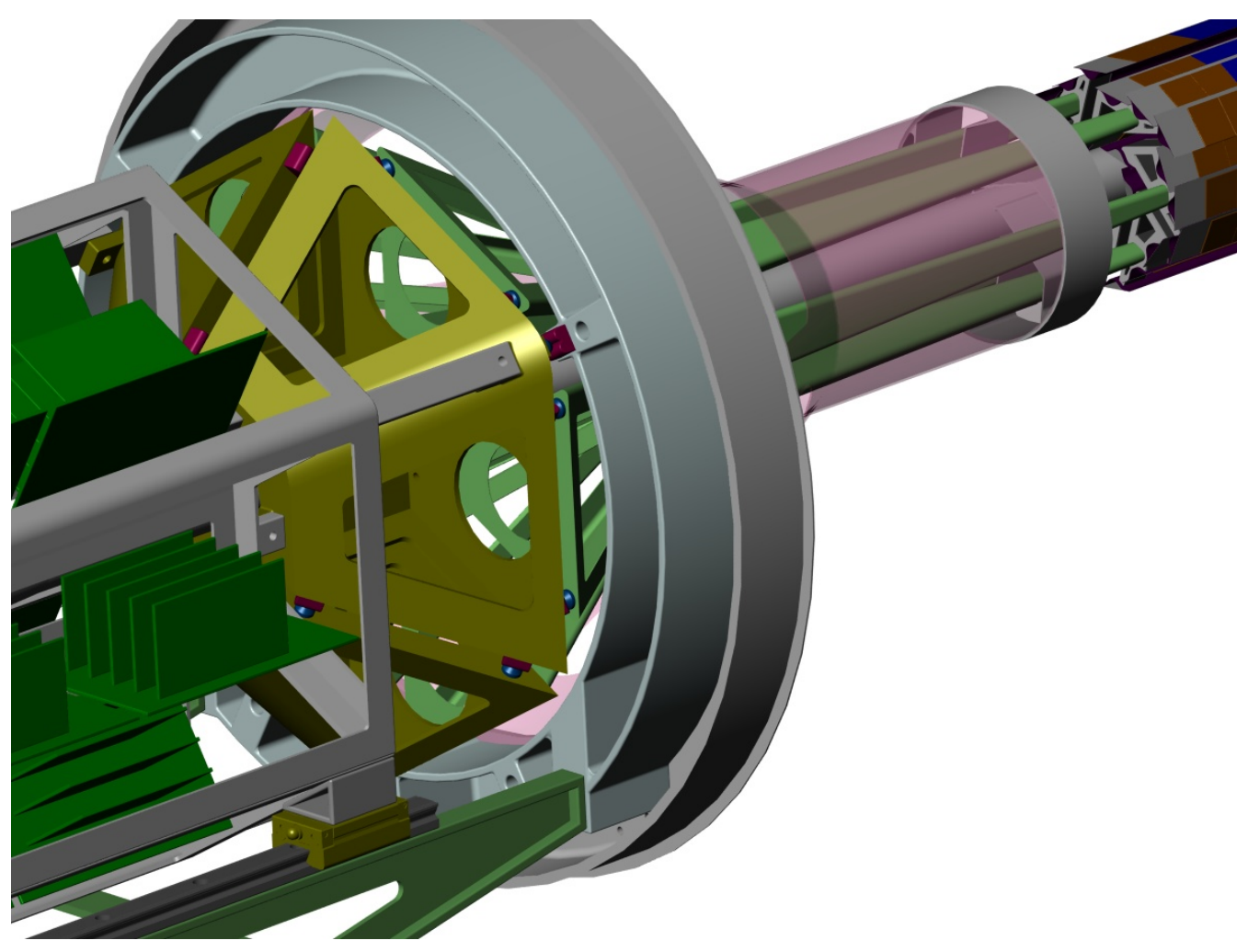

Figure 53: The HFT is shown integrated with the STAR inner detectors cone assembly.

For simplicity several existing STAR structures such as the SSD and the cone support are not shown.

\subsubsection{Position Alignment and Calibration}

The required position resolution of better than $10 \mu \mathrm{m}$ is a significant challenge for calibration and alignment. The detector system is small and so it permits a design with a single kinematic support to define a reproducible position centered on the STAR system. With the proposed mechanical design the detector system can be moved as a unit between the visual coordinate measuring system and the docking support in the STAR experiment without disturbing the relative HFT ladder positions. The brass colored structures shown in Figure 52 can be separated and reassembled without changing their relative alignment. This opening of the structure is necessary both for installation around the beam pipe and for visually accessing the inner layer detector surfaces with the visual coordinate measuring machine. The detector silicon will be patterned with a fiducial grid in the top aluminum connection layer. This fine-grained grid will be mapped in $3 \mathrm{D}$ with a visual coordinate measuring machine.

The pixel-to-pixel mapping will be preserved through transfer and docking in the middle of the STAR detector system. The calibration burden by track matching will be limited to determining the 6 parameters defining the location of the vertex detector unit within 
the other STAR detectors. It is expected that success of this approach will require careful temperature control. Further analysis and measurements will quantify this requirement.

\subsection{Ladder Design and Fabrication}

One of the ladder designs currently under evaluation is shown in Figure 54. This structure must be stiff enough to be supported at one end, only, and maintain reliable position stability to better than $10 \mu \mathrm{m}$. The ladder is made up of a $150 \mu \mathrm{m}$ two ply carbon composite open beam, which is closed with the detector sandwich to make a rigid structure. The bottom layer of the detector sandwich is an aluminum Kapton cable. Wire bonds will provide connections between the APS chip and the cable.

It was found with test structures that it is relatively straightforward to make wire bonds on these thinned devices where vacuum chucks maintain the sandwich as a flat firm surface. Alternate carrier designs are also being investigated.

A method to bond the silicon sandwich has been developed using DuPont Pyralux LF thermally activated acrylic sheet adhesive. Advantages of using sheet adhesive include fixed bondline thickness and ease of handling. Other bonding methods including low viscosity epoxies are also under investigation.

A complete ladder using silicon-sandwiches has not been produced yet but we have tested fabrication methods that use a uni-layer silicon structure. We have used vacuum chuck fixturing to butt-join chips side by side against a straight edge and have then bonded to Kapton with Pyralux. This bonded structure was then bonded at room temperature to the carbon beam using Hysol EA9396 aerospace epoxy. $75 \mu \mathrm{m}$ glass spacer beads were added to the epoxy for the initial test structure to control the bondline thickness. Prior to bonding, both the sandwich and carbon beam are quite flexible and their shape is defined during bonding with vacuum chucks.

A few $\mu \mathrm{m}$ protective polymer, Parylene, will be used to protect the exposed wire bonds and to control the spread of carbon dust. The Parylene coating process is available in the electronics industry. The coating material is applied at the molecular level by a vacuum deposition process at ambient temperature. The thickness is well controlled and it is uniform without pinholes, so protection can be achieved without compromising detector thickness. Application at room temperature avoids introducing stresses that distort the ladder shape. 


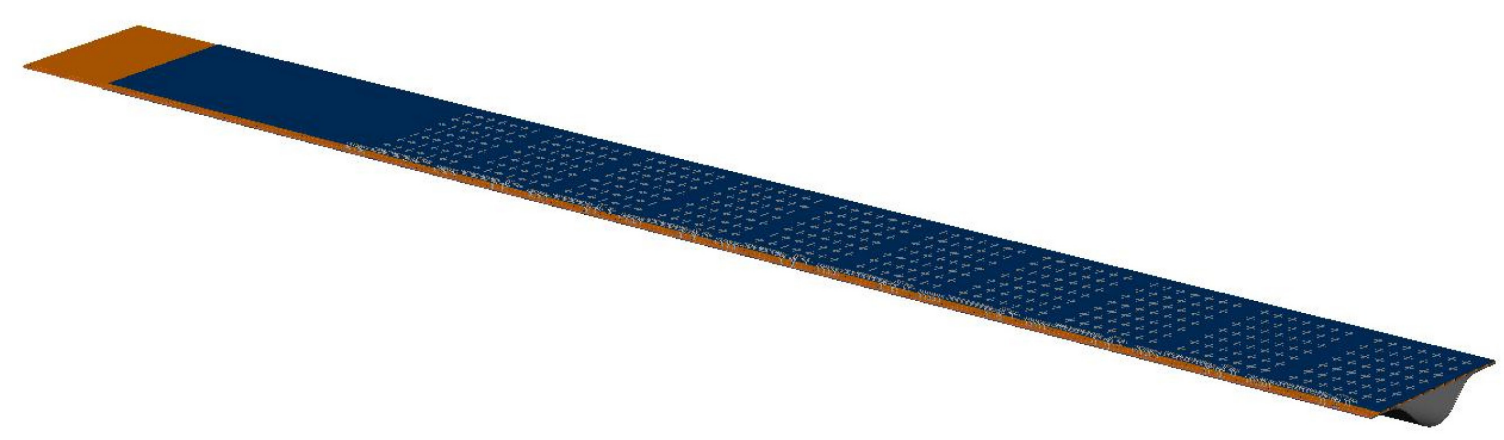

Figure 54: Ladder assembly. The current ladder is composed of a row of 10 thinned APS detectors on top of a matching row of thinned readout chips and a three-layer aluminum Kapton cable. The silicon cable structure is bonded to a carbon composite " $v "$, closing the beam to make a rigid structure.

\subsubsection{Detector Radiation Length}

Multiple scattering in the beam pipe and the first inner layer of the vertex detector sets the intrinsic limit of precision for vertex resolution. The radiation thickness for an inner ladder and the beam pipe is given in Table 19. The detector ladder is placed at an angle and is quite close to the interaction point so particles pass through the material at varying angles. This results in path length through the materials that is greater than the thickness. Table 19 shows the thickness and radiation length of a ladder and the beampipe.

\begin{tabular}{|l|c|c|l|}
\hline \multicolumn{1}{|c|}{ Material } & $\begin{array}{c}\text { Si equivalent } \\
(\mu \mathrm{m})\end{array}$ & Material thickness $(\mu \mathrm{m})$ & $\% \mathrm{X}_{0}$ \\
\hline Beryllium beam pipe & 133 & 500 & 0.14 \\
\hline \hline Silicon & 50 & 50 & 0.053 \\
\hline Adhesive & 13.4 & 50 & 0.014 \\
\hline Cable Assembly & 83.9 & 125 & 0.089 \\
\hline Adhesive & 13.4 & 50 & 0.014 \\
\hline Carbon Composite & 103 & 3200 & 0.11 \\
\hline Total for one ladder & 264 & 3475 & 0.282 \\
\hline
\end{tabular}

Table 19: Materials in the beam pipe and the first detector layer with their total thickness and radiation length. For details see Ref. [115]

\subsubsection{Expected Radiation Exposure}

RHIC luminosity projected to 2008 with the assumptions of a 21 week running period and a $60 \%$ operating efficiency for $\mathrm{Au}+\mathrm{Au}$ give a radiation dose of approximately 3.7 
$\mathrm{kRad} / \mathrm{year}$. This calculation may be found on the web ${ }^{116}$. The materials that we anticipate using in the HFT detector are the same as used in much higher radiation environments like the Atlas pixel detector. One exception is the acrylic adhesive, which exhibits excellent radiation resistance (Radiation Index $\sim 5)^{117}$. Our structure should not exhibit any mechanical degradation from the absorbed dose.

\subsection{Ladder Mechanical Tests}

\subsubsection{Load Distortion Tests}

Figure 54, and Figure 56 show a prototype of the ladder and detector carrier that we have built. Figure 55 shows a cross-sectional view of the ladder. The mechanical tests have shown that the stiffness and bending characteristics of the assembly are acceptable. For these and similar tests ${ }^{118}$, the ladder's mechanical structure was supported on one end and the surface contours were measured using the vision measuring machine at LBNL with and without a 10 gram end load. The deflection profile is shown in Figure 57 along with the calculated deflection for a simple triangular closed beam. The stiffness is within $20 \%$ of expectations from an engineering model. The measured fundamental frequency of the ladder is $140 \mathrm{~Hz}$ while the engineering model gives a resonant frequency of $135 \mathrm{~Hz}^{119}$. We have also measured the vibrational environment at the STAR detector and it is dominated by low frequencies, $\sim 1 \mathrm{~Hz}$, and so we anticipate that the ladder will maintain its position to better than a few microns.

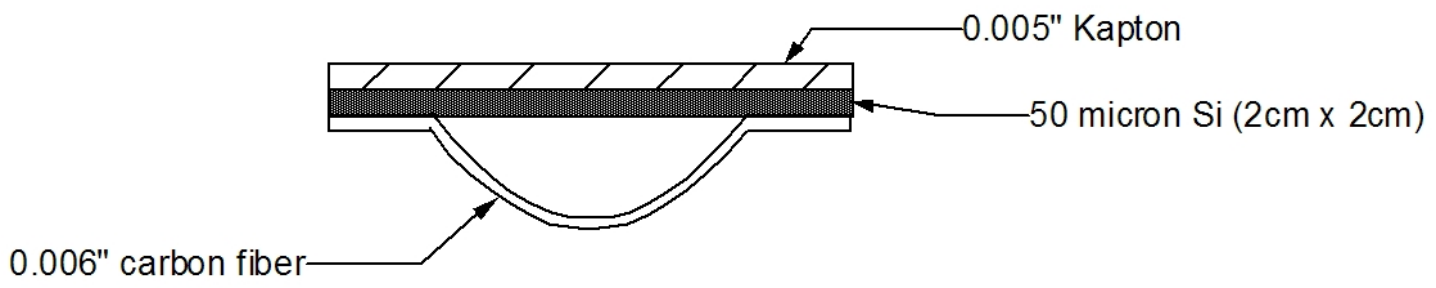

Figure 55: A cross-section of the prototype detector ladder showing its structure and materials composition. 


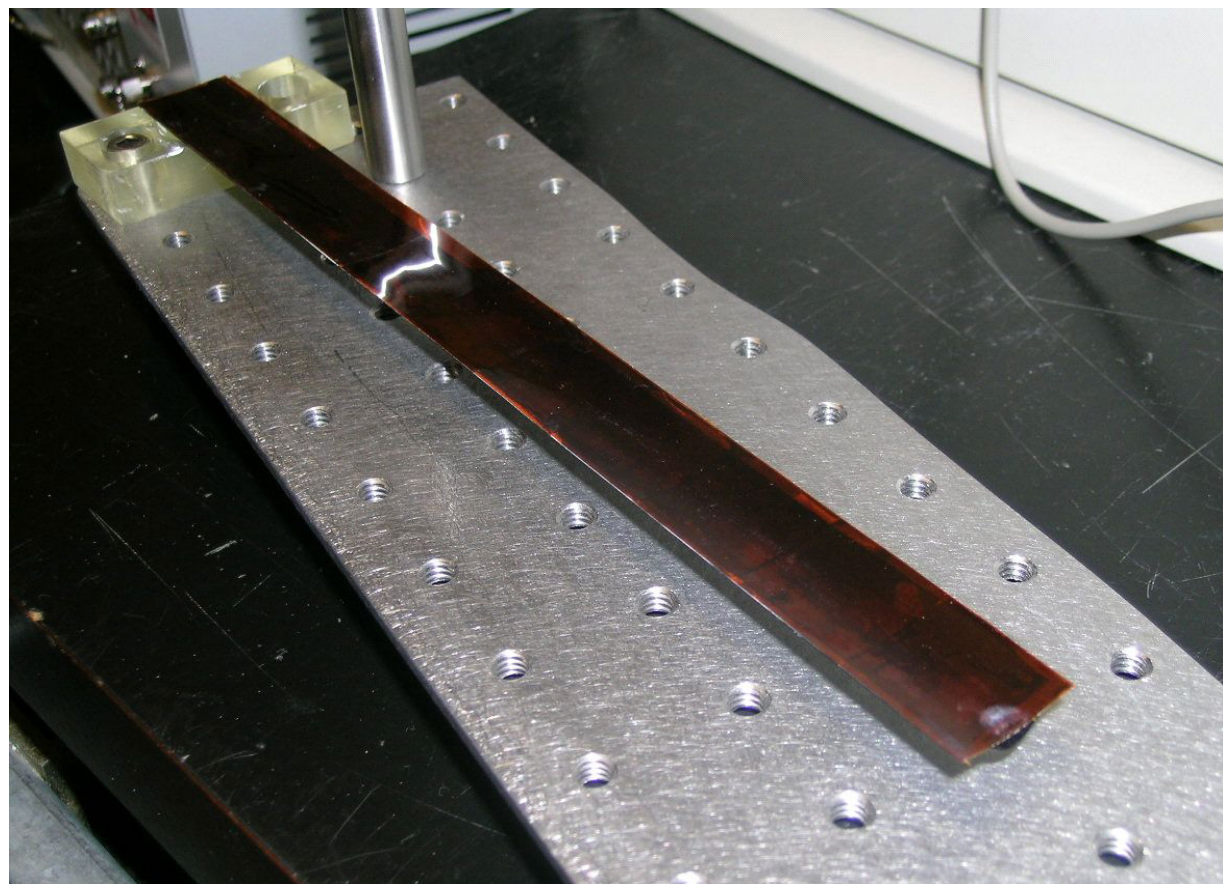

Figure 56: A cross-section of the prototype detector ladder showing its structure and materials composition.

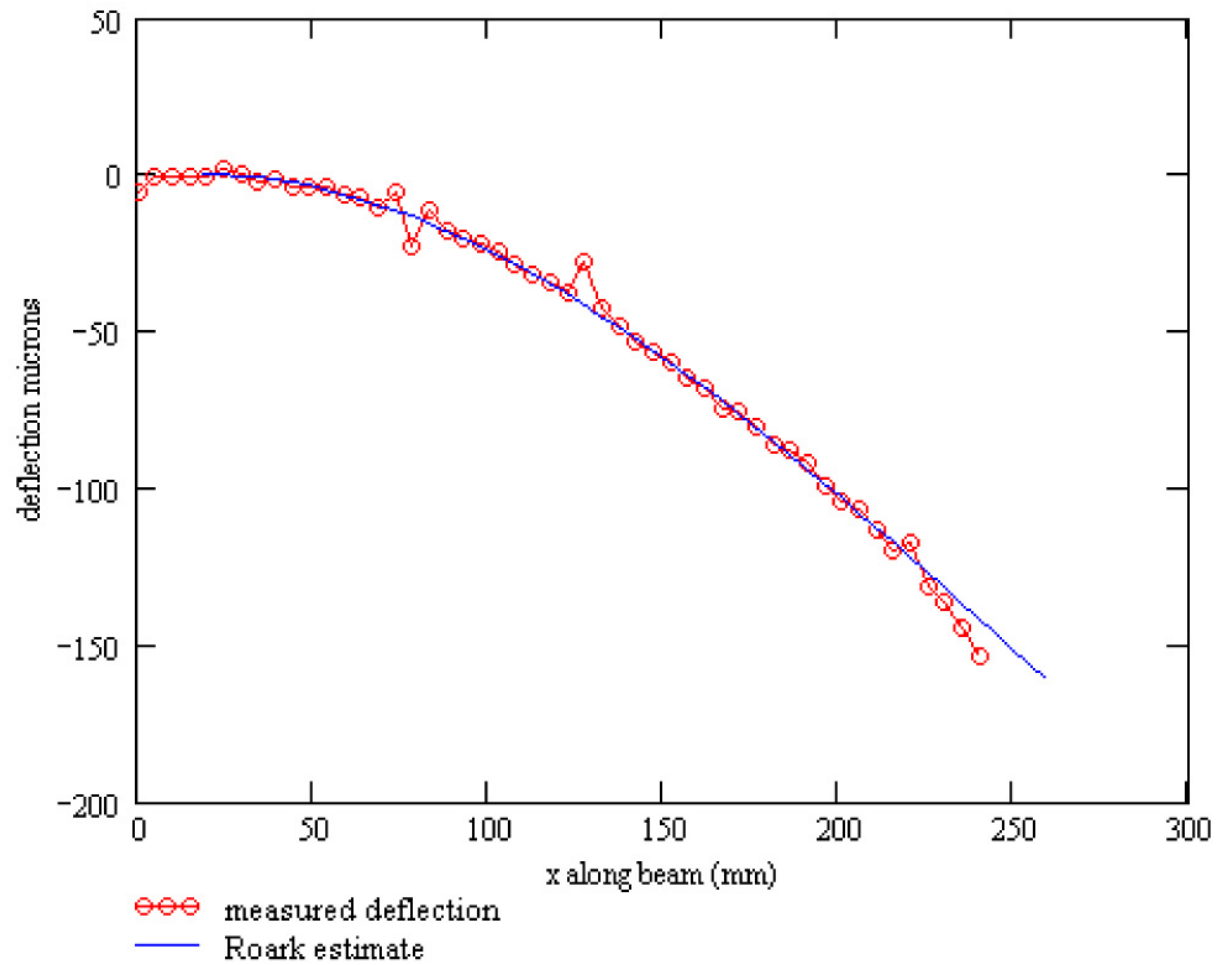

Figure 57: Measured bend of a silicon/carbon composite ladder test structure and the calculated bend shape. The ladder was rigidly supported at one end with a 10 gram weight placed on the other. 


\subsubsection{Thermal Distortion Tests}

We are in the process of measuring thermally generated deflections using TV holography $^{120}$. This tool, available to us, courtesy of the ATLAS Pixel project, provides a rapid visual distortion map on the sub micron scale. This is proving to be a useful tool for identifying and understanding different design parameters. Measurements are done on structures thermally isolated in a transparent box (see Figure 58). This tool will also be used to test the stability of the final detector.

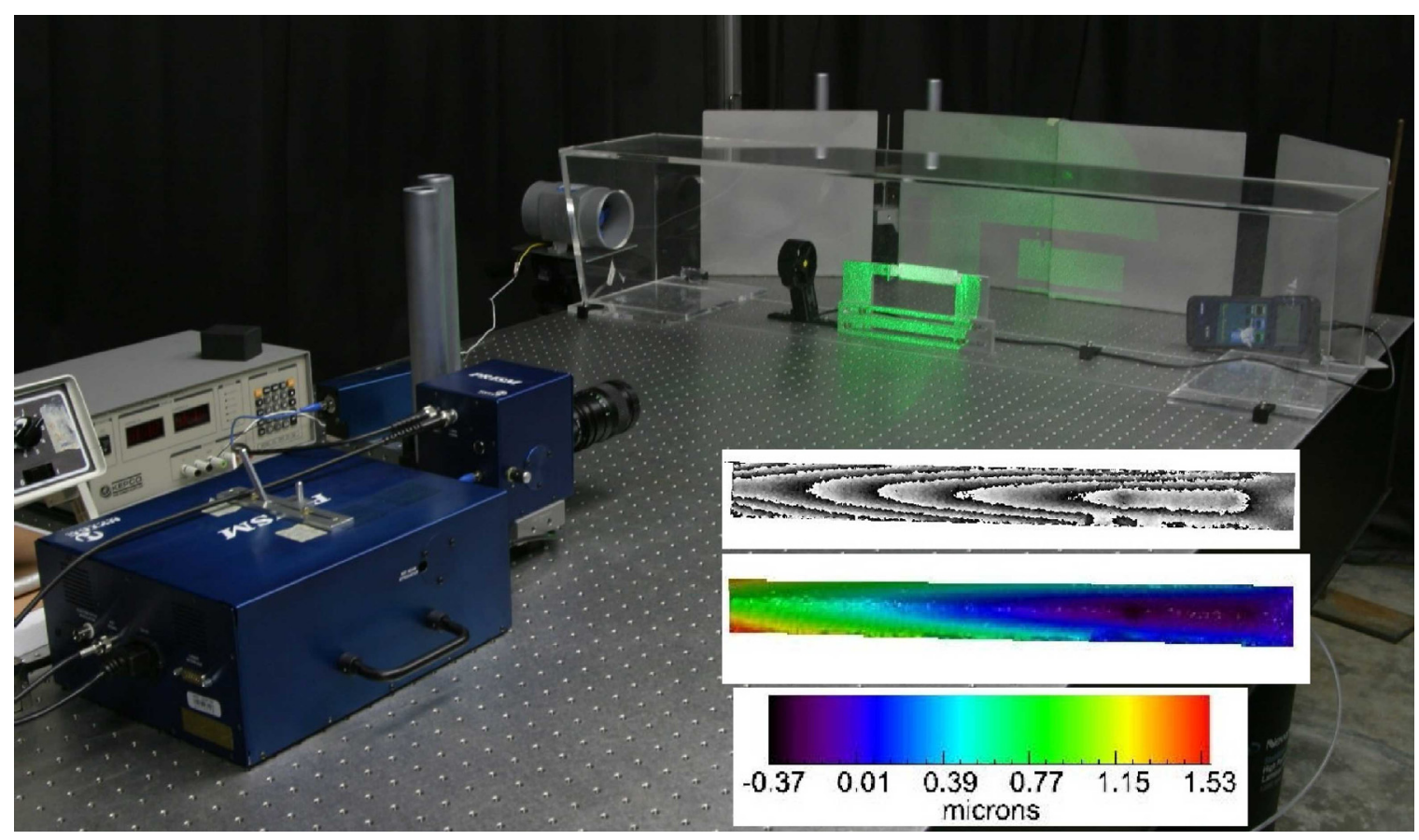

Figure 58: TV Holography system viewing test ladder in a small transparent wind tunnel. Inset shows diffraction pattern with color map of the surface displacement.

\subsubsection{Cooling Measurements}

In the interest of low mass, the detector system is being designed to use air-cooling for the detector structure in the active tracking volume. A small wind tunnel has been constructed (see Figure 58) to evaluate the cooling capacity of air and the design of the system. Measurements with resistive heated ladder test structures and thermocouple readouts show that air velocities on the order of $1 \mathrm{~m} / \mathrm{s}$ are sufficient to handle power levels of $150 \mathrm{~mW} / \mathrm{cm}^{2}$. This setup, with its thermal camera, will be used to check operating silicon-ladder-devices to evaluate localized heating issues. In addition to cooling tests the wind tunnel is being used to evaluate the vibration stability of the ladder design under the required wind flow conditions. The amplitude of cooling air driven vibrations in the ladder is measured with non-contact capacitive probes ${ }^{121}$ and if 
necessary specific vibration modes can be monitored with the TV holography system. The vibration induced by the air cooling gives an acceptable positional location $\sigma$ of 1.6 $\mu \mathrm{m}$. The low mass ladder structure is the critical design element requiring vibration testing, but the full detector structure will be evaluated with this system as well ${ }^{122}$. Additional information on the cooling studies is available on the web ${ }^{123}$.

\subsection{Cabling}

Connection to the detector readout chips will be done with an aluminum Kapton cable. The cable is part of the ladder and is located underneath the APS detector. It will carry power, clock, analogue signals and control. We have recently found a commercial vendor that makes aluminum cables with vias. Fabrication tests have been done with 0.35 mil aluminum with 1 mil Kapton plus two 1 mil acrylic adhesive layers. Wire bonding tests with this material have been successful.

Figure 59 shows the prototype for a HFT readout cable. The prototype is an active cable with a buffer and differential amplifier for each sector of 10 MIMOSA detectors for use in testing the MIMOSA-5 detectors. The final detector readout cable will not have the components shown but will narrower and will be sized to be slightly wider than the detectors themselves to allow wire bonding. The cable in the figure uses a copper conductor on a $25 \mu \mathrm{m}$ Kapton insulator. The final cable will be a 4-layer low-mass aluminum conductor cable with a radiation length equivalent of $84 \mu \mathrm{m}$ of Si. The prototype has been successfully tested and is working in our prototype ladder readout system. Additional and more recent information on cable and ladder developments may be found on the web ${ }^{124}$.

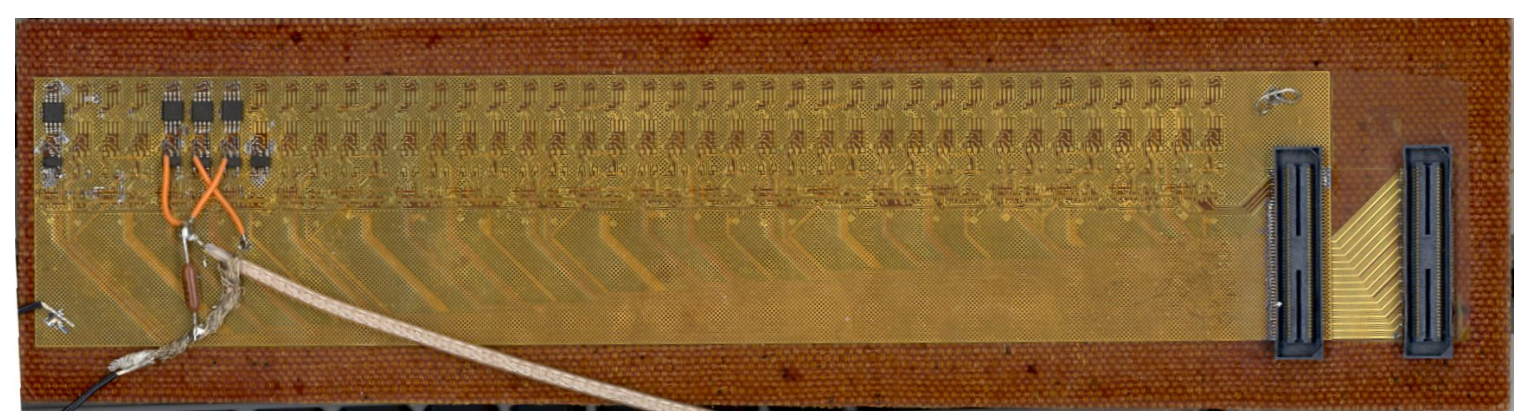

Figure 59: A prototype readout cable for the HFT.

Figure 60 shows a mechanical Prototype with 4 MIMOSA-5 detectors glued to the Kapton cable assembly with a thin acrylic film adhesive. The adhesive has a bond line of $50 \mu \mathrm{m}$. The MIMOSA is relatively flat in horizontal direction but has a "dish" shape in vertical (y) direction. The magnitude of these distortions is, however, quite small. The largest deviation from a linear fit in $\mathrm{y}$ is only $\pm 6 \mu \mathrm{m}$. This low rate of deformation will 
allow us to make a minimum number of measurements of the detector positions on the ladders in order to locate the individual pixel position to the required accuracy by a parameterized location function ${ }^{125}$.

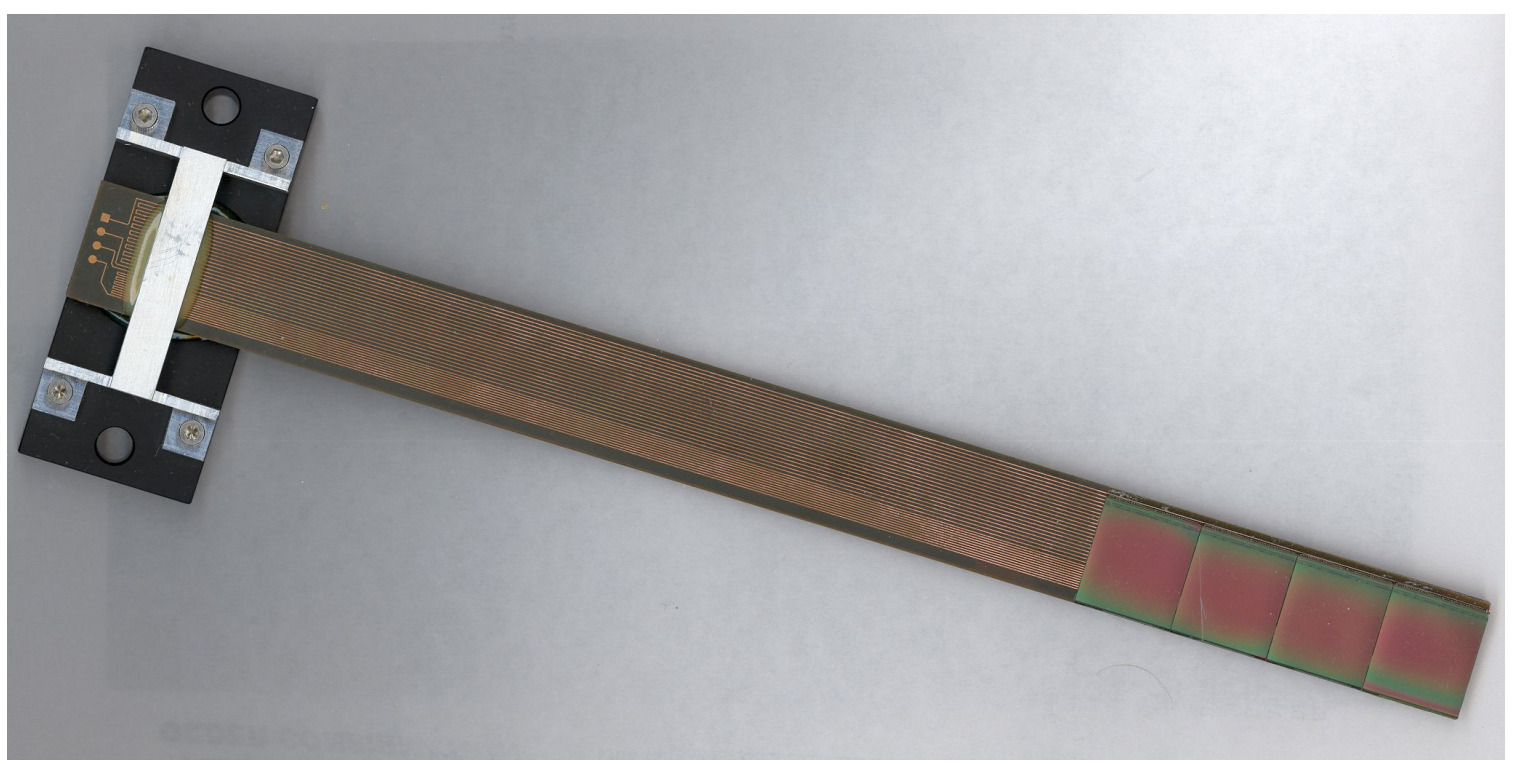

Figure 60: Mechanical Prototype with 4 MIMOSA-5 detectors glued to the Kapton cable assembly. 


\subsection{Beam Pipe}

The vertex detector extends the measurement radius to $15 \mathrm{~mm}$ from the interaction axis and will require a new small radius beam pipe for STAR. The design concept for this pipe is shown in Figure 61.

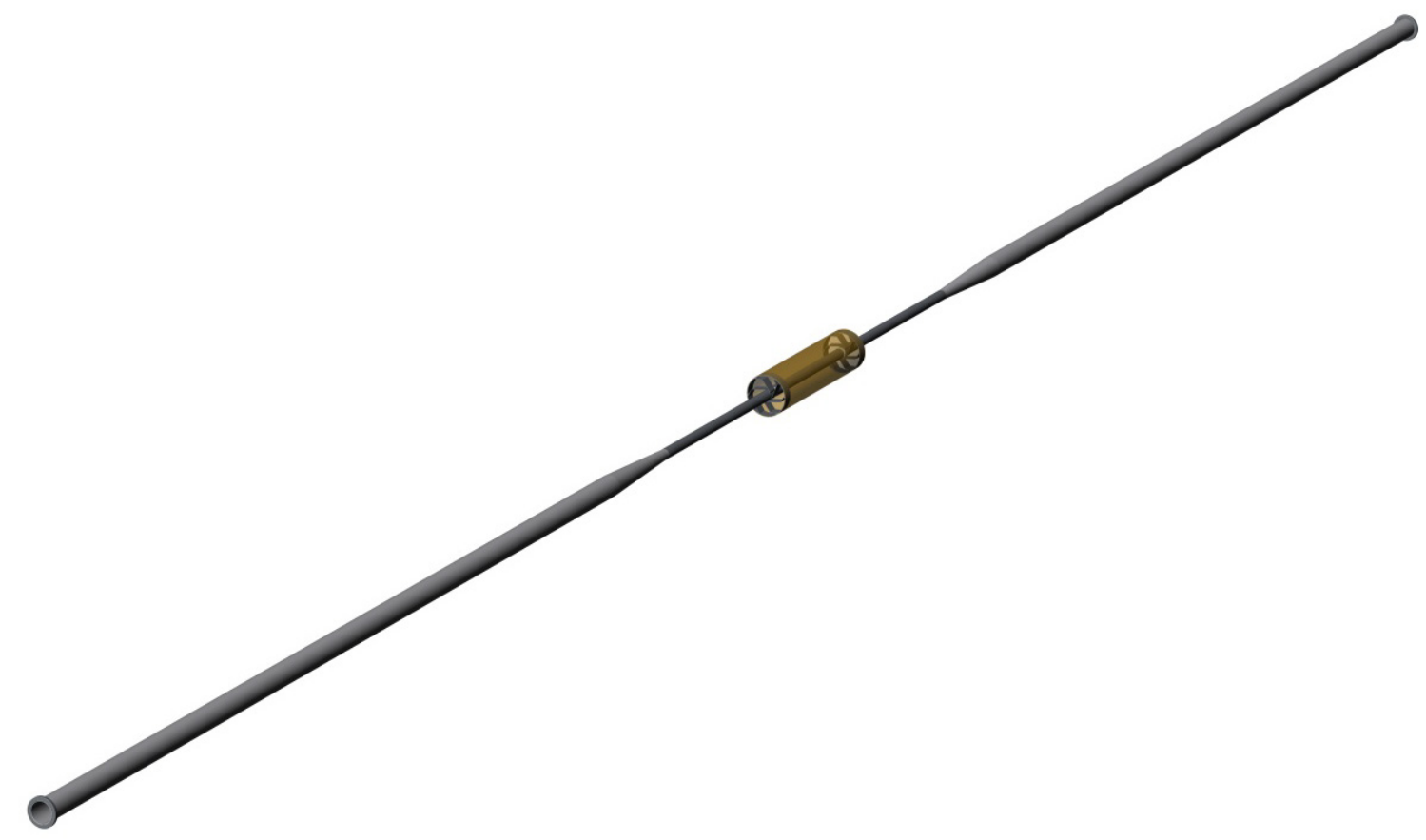

Figure 61: Beam pipe structure with outer support barrel in the center.

The beam pipe is constructed from $60 \mathrm{~mm}$ diameter aluminum tubes that taper down to a $29 \mathrm{~mm}$ diameter beryllium beam pipe with $1 \mathrm{~mm}$ thick walls. The central region where the HFT is located is a $29 \mathrm{~mm}$ diameter beryllium pipe with a $0.5 \mathrm{~mm}$ thick wall. An exoskeleton made up of an outer tube and the two spoked wheels carries the support load, isolating the thin central tube such that it only has to carry vacuum load. Central support of the beam pipe system against gravity is provided by securing the exoskeleton structure. The central portion with the exoskeleton structure is shown more clearly in Figure 62. The outer shell has been made transparent in the figure to show the inner structure. 


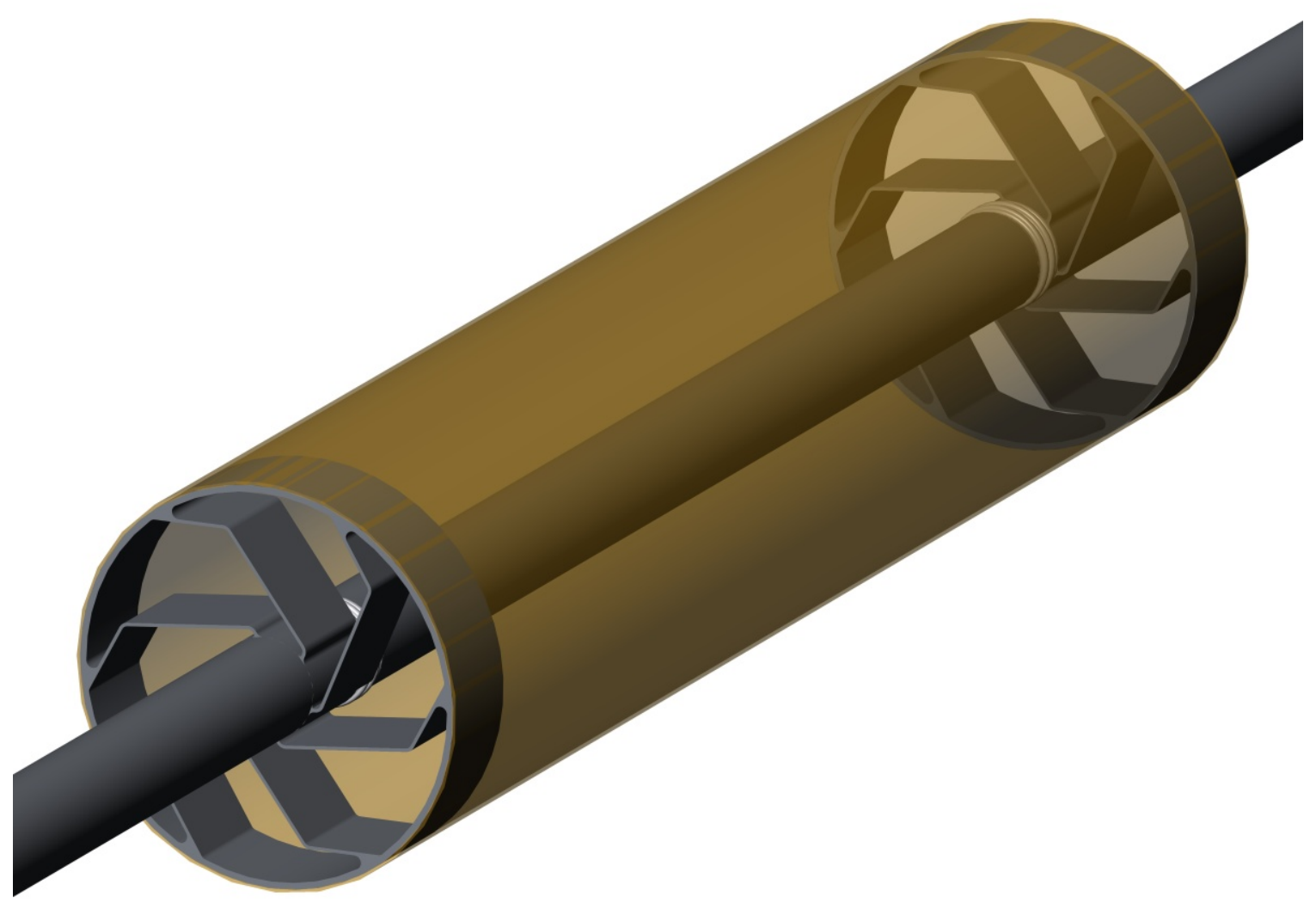

Figure 62: View of the beampipe mid-section.

\subsubsection{Minimum Beam Pipe Radius Consistent with Injected Beam}

We have chosen a minimum radius for the beam pipe which, now that the RHIC optics have stabilized, is reasonable conservative. From the standpoint of beam pipe interaction with beam optics, the beam pipe wall should, according to accelerator physicists at BNL, have a minimum radius of $6 \sigma$ while $10 \sigma$ is very conservative ${ }^{126} . \sigma$ is the beam envelope at injection. For STAR at injection the beam emittance is $15 \pi \mathrm{mrad} \cdot \mathrm{mm}, \beta^{*}$ is $10 \mathrm{~m}, \beta \gamma$ is 10.52 which gives $\sigma=1.5 \mathrm{~mm}$ plus a $5 \mathrm{~mm}$ beam offset at injection ${ }^{126,127,128,129}$. For protons injection $\sigma$ is smaller and therefore less of a constraint. We have chosen an inner beam pipe radius of $14.5 \mathrm{~mm}$ or $\sim 7 \sigma$. This is satisfactory for RHIC operation, however, depending on focusing details this could be the limiting aperture, leaving the HFT vulnerable to uncontrolled beam dumps ${ }^{129}$.

\subsubsection{Beam Pipe Radius - Vacuum Considerations}

The dimensions of the beam pipe sets limits on pumping speed and the expected pressure at the center of the interaction region in STAR. The $1600 \mathrm{~mm}$ long central section with a $28 \mathrm{~mm}$ diameter joins a larger $58 \mathrm{~mm}$ diameter pipe, which extends $3000 \mathrm{~mm}$ to the pumping section. A simple analysis was performed ${ }^{130,131,132}$ which gives a pressure 
increase at the center in the interaction region of $\sim 10^{-11}$ torr. This is the pressure increase above the pressure at the pumping station and it is based on a surface out-gassing rate of $5.3 \times 10^{-11} \mathrm{~W} / \mathrm{m}^{2}$ and a conservative estimate of the pipe conductance ${ }^{131}$. This is well within the requirements. The greatest uncertainty in this estimate is the out-gassing rate, but a factor of 10 greater value is still tolerable. If an Active NEG coating is used the inner section will be a pumping surface rather than an out gassing surface.

\subsubsection{Supporting Section}

The central supporting section will be attached to the existing carbon cone in STAR and will provide the support for the center portion of the beam pipe. The outer cylinder and spoke section, made of either carbon composite or beryllium, is designed to support the pipe sections extending from either end and constrain them sufficiently that a very thin beam pipe structure can be used in the center region. This outer cylinder with spokes plus flex sections will isolate the thin central pipe from outside forces so that the central pipe only has to support the vacuum load. The spokes that couple the extended beam pipe to the outer cylinder are shaped to allow free insertion of the vertex detector into the enclosed center region. The end view in Figure 63 shows how the detector ladders mesh with the support spokes.

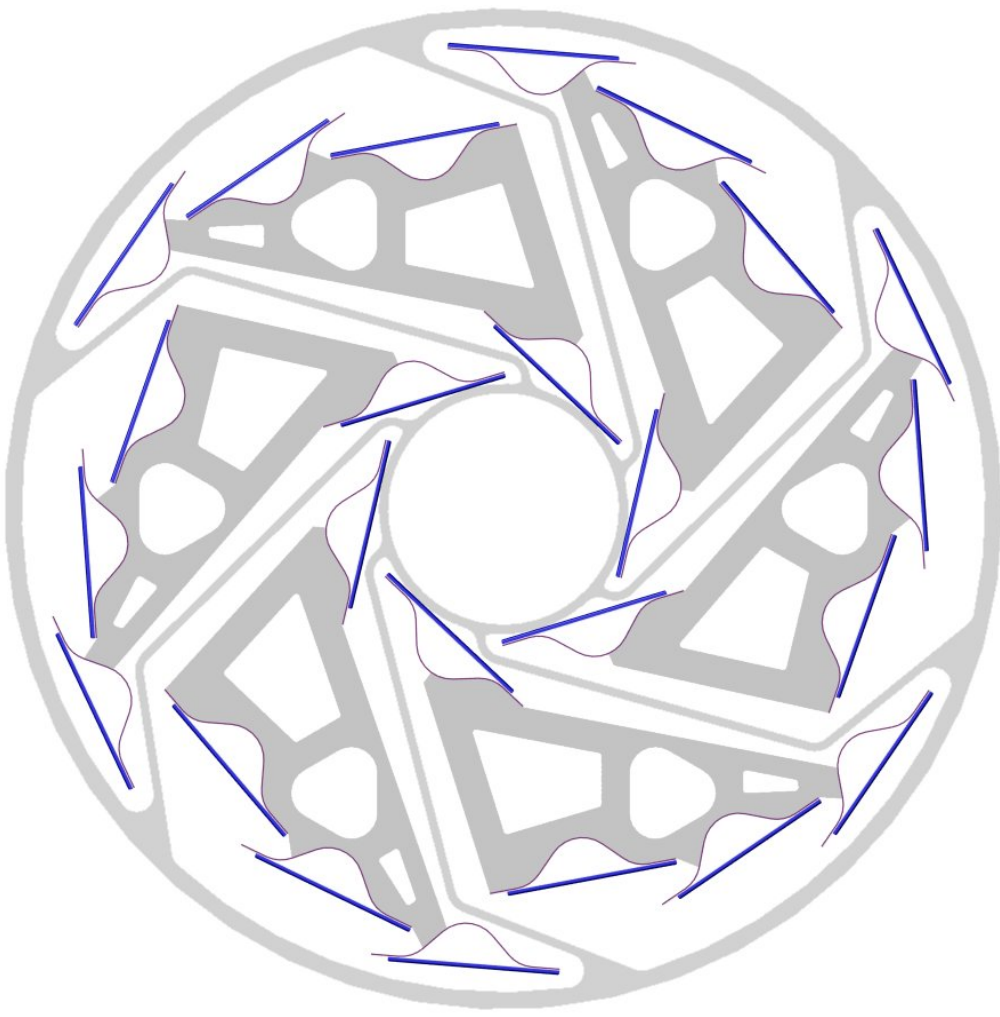

Figure 63: End view showing the HFT ladders between spokes to the inner beam pipe support. 


\subsubsection{Central Beam Pipe Thin Window Section}

The current plan for the thin central region is to use a beryllium pipe with a $0.5 \mathrm{~mm}$ wall thickness. It is believed that this will provide the minimum coulomb scattering while maintaining a reasonable structural safety margin. Preliminary analysis indicates a 50:1 safety margin for buckling ${ }^{133}$. There is a structure failure limit imposed by the degree of roundness of the cylinder. A stress analysis indicates that a $1 \mathrm{~mm}$ deviation from a cylinder ( $1 \mathrm{~mm}$ difference between major and minor axis of an ellipse) sets a 20:1 safety margin for material failure.

\subsubsection{RF Background from the Wake Field}

It is believed that the beam pipe in STAR will be sufficient to suppress wake field signals in the detector to well below the expected signal. The evidence for this belief comes from experience with the gas jet polarimeter and carbon strip polarimeter located at the 12 o'clock intersection region of RHIC. These polarimeters have silicon detectors located inside the beam pipe close to the beam and it has been found that a few microns of conductor are sufficient to shield these detectors from the wake field ${ }^{134}$.

The HFT will be located outside the beam pipe and will benefit from the RF shielding this pipe provides. The pipe will be constructed from at least 500 microns of beryllium. The maximum beam in RHIC will be $1 \times 10^{9}$ gold ions per bunch. This gives a wake field current of $110 \mathrm{~mA}$ (counting both beams), which with a $1 \mu \mathrm{m}$ skin depth will generate a resistive potential drop of only $7 \mathrm{mV}$. A more sophisticated calculation should include induction, rise time and skin depth and detector filtering, since our bandwidth cutoff will be closer to the wake field $\mathrm{GHz}$ frequency than the polarimeter detector cutoff.

\subsubsection{Beam Pipe Insertion and Removal}

We are currently investigating the removal and insertion of the beampipe. We anticipate replacing the current center section of the STAR beampipe with the components described above. This will likely entail a period of refit and installation to install the new beampipe after which removal and reinsertion should not be necessary during the normal course of running and detector repair.

\subsubsection{Bake Out}

Beam pipe bake out is still under discussion. It is expected that the same matter NEG coating will be used and there are two possibilities in how this will be treated. It may either be a minimum bake out to remove primarily water as it is done now with a hot dry nitrogen scrub gas at $150 \mathrm{C}$ to $110 \mathrm{C}$ or it could be a full activation of the NEG coating turning it into a pumping surface ${ }^{135}$. This later case requires baking at $150 \mathrm{C}$ to $200 \mathrm{C}$ 
with vacuum. This could impose additional constraints on beam pipe materials and would require additional thermal isolation from the other detectors. If it is decided that the less aggressive bake out procedure is adequate then carbon composites can be used in the construction of the beam pipe exoskeleton. In either case the HFT will be withdrawn during bake out from the operating location to isolate it from the heated beam pipe.

\subsection{Compatibility with the SSD and other cone mounted detectors}

The HFT will be supported and installed from one end only. There will be no other detectors requiring support or access at this end. Other detectors requiring cone support will be outside of the HFT space. The FTPC on one end will have to be removed to accommodate the HFT and support electronics as shown in Figure 49.

\subsection{Compatibility with an Upgraded Inner Si Tracker Barrel}

If the SVT is replaced with a new inner Si Tracker barrel and forward disks for the tracking outside of the SVT then the support structure for the new tracker will be installed from one end of STAR and the HFT will be supported and installed from the opposite end. This approach is being further developed by our group and the MIT Si barrel group. 


\section{Preliminary Cost and Schedule Estimates}

\subsection{Cost and Schedule}

The HFT is a very demanding technical project and it requires a substantial amount of $\mathrm{R} \& \mathrm{D}$ in order to ensure that it will be ready to take data when RHIC is running. The enclosed cost and schedule estimates reflects the need to develop and test the pixel sensors over a period of several years. We envision at least four generations of chips before we have a full sized, production quality, chip design. The cost and schedule also reflects the need for replacement parts so that the detector can be easily repaired during a run.

Synchronizing with the RHIC run schedule is beyond the scope of this document, but it is an important topic none-the-less because a successful HFT program requires several years of running, including (as a minimal set) a high statistics top energy Au-Au run, a p$\mathrm{p}$ run, and a d-Au run. Since the RHIC schedule is hard to predict, it is very important that the HFT be ready on time, and be reliable during each run. The number of opportunities to run top energy $\mathrm{Au}-\mathrm{Au}$ beams are very limited. Therefore, we have designed the HFT to be easily repaired during a run and even replaced, if necessary.

A schematic overview of the proposed $R \& D$ and construction schedule is shown in Figure 64 with particular emphasis on the installation dates. Note, for example, that the figure includes a milestone for the installation of a detector telescope based on MimoSTAR II chips in the summer of '06. The detector telescope will take data in FY07 with the goal of testing the APS chip technology under working conditions at the STAR experiment. Additional installation activities are shown in each year. A more detailed roll-up of the projects activities is shown in Figure 65.

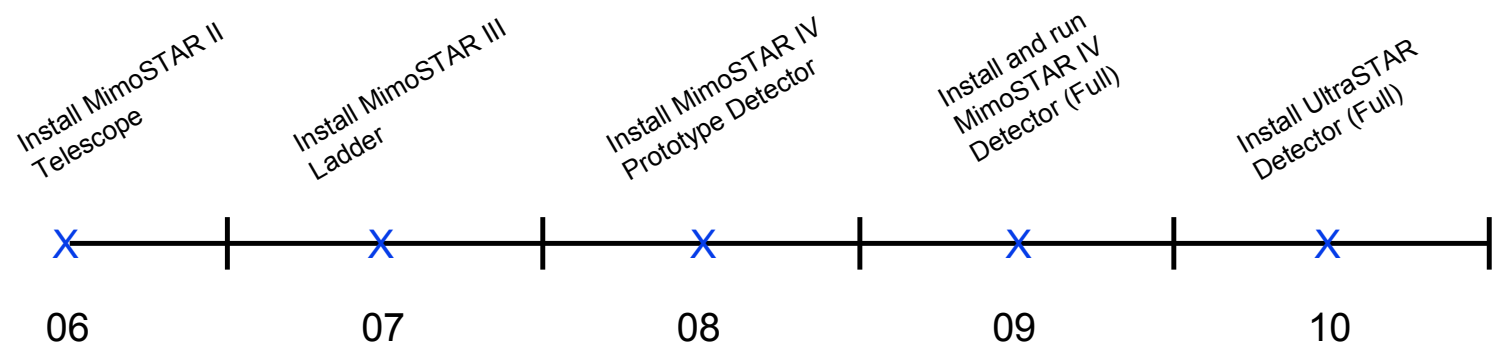

Figure 64: A Schematic view of the installation activities in each year. Installation is typically done in the summer of each year. 


\begin{tabular}{|c|c|c|}
\hline & (6) & Task Name \\
\hline 1 & & $\square$ HFT \\
\hline 2 & & $\boxplus$ Detector chips \\
\hline 10 & & $\mp$ Detector Cables \\
\hline 14 & & $Ð$ Readout Boards \\
\hline 19 & & † DAQ Interface \\
\hline 23 & 国 & installation in beam test1 \\
\hline 24 & & $\mp$ Monitor and Control Software \\
\hline 27 & & $\boxplus$ Ladder \\
\hline 32 & & $\mp$ Ladder Support \\
\hline 38 & & † Kinematic Mount \\
\hline 43 & & $\Phi$ Card Cage \\
\hline 46 & & $\boxplus$ Carriage \\
\hline 49 & & $\oplus$ Cone \\
\hline 53 & & $\oplus$ Cooling System \\
\hline 57 & & \pm Metrology \\
\hline 61 & & $\boxplus$ Beam Pipe \\
\hline 64 & & $\mp$ Beam Pipe Insertion tool \\
\hline 67 & & $\mp$ Detector Bench Test \\
\hline 70 & & $\oplus$ Power \\
\hline 72 & & $\boxplus$ Installation \\
\hline 75 & 国 & Installation complete $\mathrm{Au}+\mathrm{A} u$ run \\
\hline 76 & & $\boxplus$ In STAR test and calibrate \\
\hline 79 & & $\mp$ Magagment \\
\hline 82 & & $\boxplus$ Software \\
\hline 86 & 国 & project funds start FY09 \\
\hline 87 & 国 & start FY10 \\
\hline 88 & 国 & project funds end FY10 \\
\hline 89 & & †U Detector Chips \\
\hline 95 & & $\oplus$ U Detector Cables \\
\hline 98 & & $\boxplus$ U Readout Boards \\
\hline 102 & & $\oplus$ U DAQ interface \\
\hline 105 & & $\mp U$ Monitor and control software \\
\hline 108 & & $\Phi$ U Ladder \\
\hline 113 & & $\boxplus$ U Ladder Support \\
\hline 118 & & $\boxplus$ U Card Cage Mod \\
\hline 121 & & $\boxplus U$ Cooling System Mod \\
\hline 124 & & $\boxplus$ U Metrology \\
\hline 128 & & $\boxplus$ U Detector Bench Test \\
\hline 131 & & $\oplus \mathbf{U}$ Installation \\
\hline 134 & 国 & Ultinate Installed \\
\hline 135 & 间 & RHIC beam on \\
\hline 136 & & $\oplus U$ In STAR test and calibrate \\
\hline
\end{tabular}

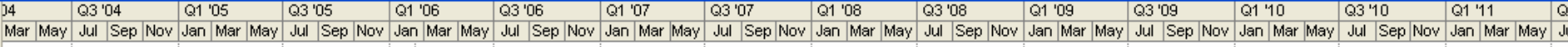

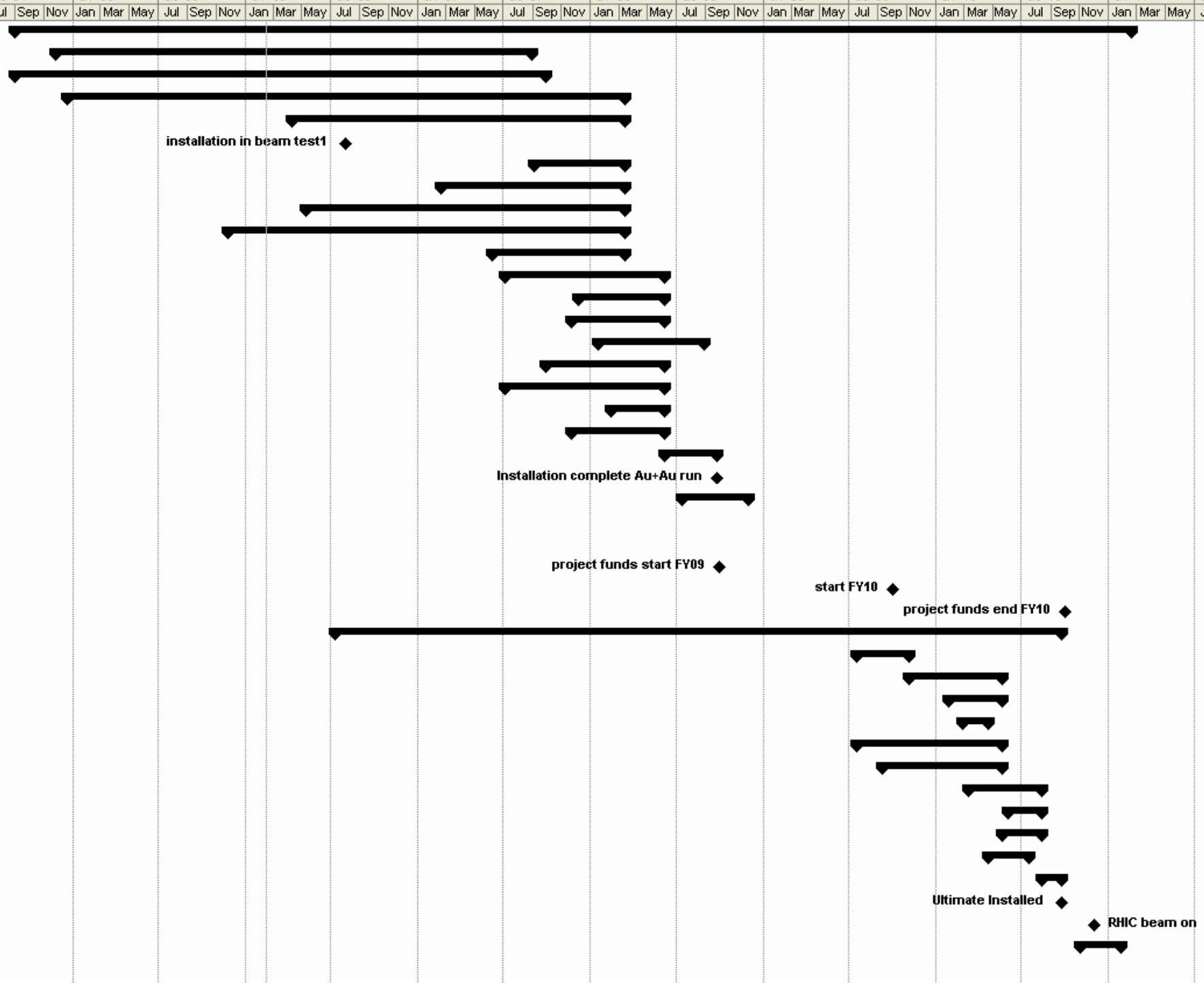

Figure 65: Schedule overview and roll-up of activities. 
A full Micro-Soft-Project model for the HFT project has been developed and the files are available on the WWW. The files are too complex to be included in this document but an extract of one of these files is shown in Figure 66.

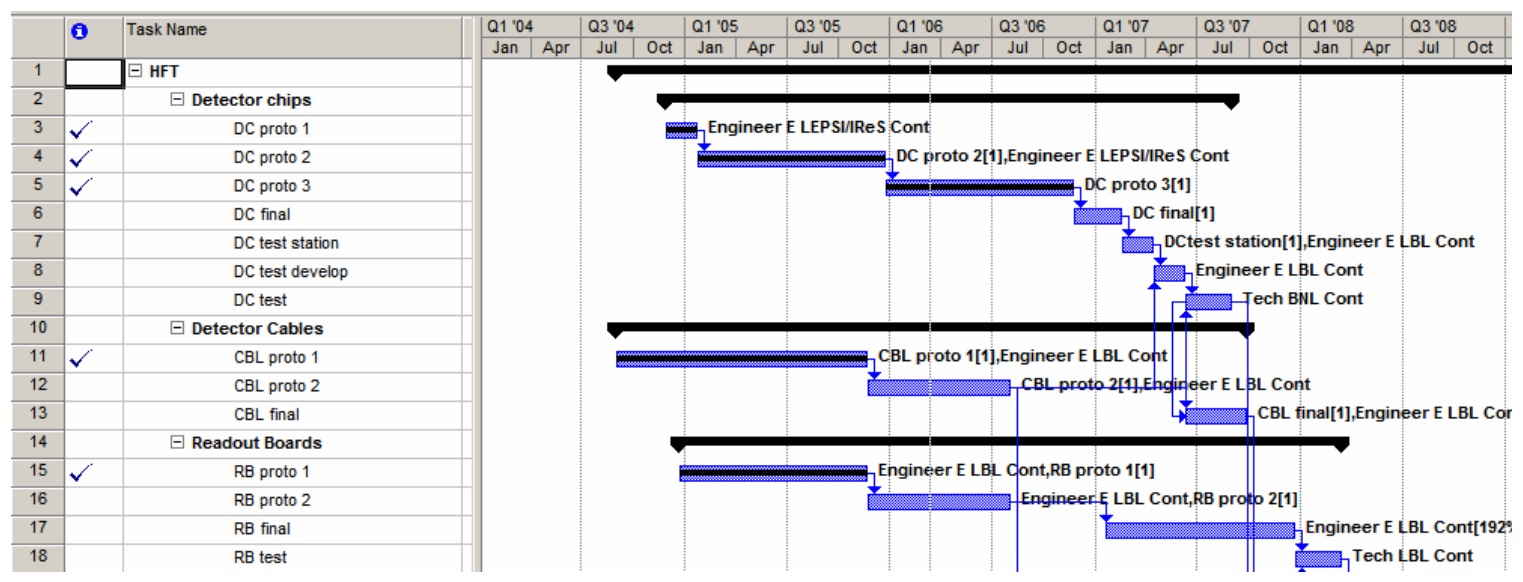

Figure 66: Detail from the Gantt Chart that tracks the full enterprise of HFT activities.

The HFT requires a substantial amount of $R \& D$ work due to the innovative new technology that is an integral part of the project. The items needing further development include the Si chips, the readout and DAQ electronics, the mechanical arms to hold and insert the detector, the beam pipe, and the calibration and tracking software. The R\&D activities will begin in FY06 but the bulk of the R\&D activities will take place in FY07 and FY08. During FY06, the engineering labor will be contributed by the Berkeley Lab, while in FY07 and 08, we anticipate that one mechanical engineer and one electrical engineer will be funded by the R\&D dollars associated with the HFT project.

The contributed engineering estimates shown in the tables, below, have not been negotiated with LBL or BNL management and are subject to further refinement and the availability of funds.

Significant R\&D activities to be studied in FY06 include:

- QA and test of MimoSTAR II chips from Strasbourg

- Conceptual design of readout boards for the Strasbourg chips

- Prototype ladders to support the chips

- DAQ interface prototype

- Develop Al clad cable technology

- Conceptual design of the kinematic mounts to hold the ladders

- Live beam tests in STAR using MimoSTAR II chip

- $\quad$ R\&D for the MimoSTAR III chip 
Estimated expenses in FY06:

- $\sim 150 \mathrm{~K}$ Procurements

- $\sim 150 \mathrm{~K}$ Engineering

- $\sim 500 \mathrm{~K}$ Contributed Engineering

Significant R\&D activities to be studied in FY07 include:

- QA and test of MimoSTAR III chips from Strasbourg

- Prototype readout boards for the Strasbourg chips

- Prototype ladders to support the chips

- Conceptual design of the kinematic mounts to hold the ladders

- Live beam tests in STAR using MimoSTAR II chips

- Collaborative design of the beam pipe

Estimated R\&D expenses in FY07:

- $\sim 670 \mathrm{~K}$ Procurements

- $\sim 250 \mathrm{~K}$ Engineering

- $\sim 1000 \mathrm{~K}$ Contributed Engineering

Significant activities to be studied in FY08 include:

- QA and test of the MimoSTAR IV chips from Strasbourg

- Live beam tests in STAR using MimoSTAR III and prototype kinematic mounts

- Integration studies for the support of the HFT and Cone modifications

- Development of alignment and calibration techniques

- Design and test of the thin walled beam pipe and analysis of risk

- Develop and test interface to STAR DAQ

- $\mathrm{R} \& D$ for the UltraSTAR Chip

Estimated R\&D expenses in FY08:

- $\sim 800 \mathrm{~K}$ Procurements

- $\sim 900 \mathrm{~K}$ Engineering

- $\sim 1000 \mathrm{~K}$ Contributed Engineering 


Item
BP procur
CA fab
CBL final
CBL proto 1
CBL proto 2
CC fab
CONE procur
COOL procur
DAQ Purch
DC final
DC proto 1
DC proto 2
DC proto 3
DCtest station
Insertion parts
Installation procur
KM final
KM proto
L final
L proto
LS final
LS proto
M fab
Monitor purch
Power
RB final
RB proto 1
RB proto 2
slow control Purch
Software Purch
Software training
TT board
U CBL final
U CBL final
U CBL proto 1
U CC design
U CC fab
U COOL mod
U DAQ final
U DAQ proto
U DC Final
U DC Proto 2
U DC test batch 1
U DT test
U L final
U L proto
U LS final
U LS proto
U M measure
U RB final
U RB proto 1

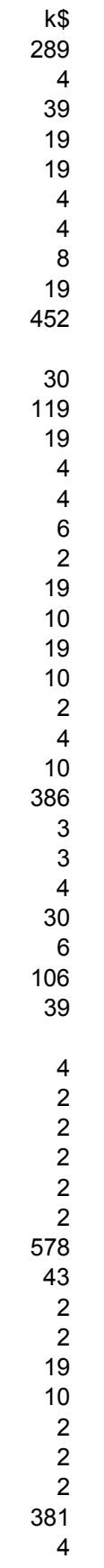

1009
Description

Beam Pipe Procurement

$\$ 288,750.00$

Detector insertion carriage fabrication

$\$ 3,850.00$

Ladder flex cable electronic substrate final fabrication

$\$ 38,500.00$

Ladder flex cable electronic substrate prototype 1

Ladder flex cable electronic substrate prototype 2

$\$ 19,250.00$

$\$ 19,250.00$

$\$ 3,850.00$

$\$ 3,850.00$

$\$ 7,700.00$

$\$ 19,250.00$

Support cone alterations for detector support

Cooling system procurement

DAQ hardware costs, PCs

Detector chip final fabrication

$\$ 451,687.50$

Detector chip prototype 1

Detector chip prototype 2

Detector chip prototype 3

Detector chip test station

Beam pipe and detector support insertion parts

$\$ 29,700.00$
$\$ 118,800.00$

$\$ 19,250.00$

$\$ 3,850.00$

$\$ 3,850.00$

$\$ 5,775.00$

Kinematic mount structures, fabrication

$\$ 1,925.00$

$\$ 19,250.00$

$\$ 9,625.00$

$\$ 19,250.00$

$\$ 9,625.00$

$\$ 1,925.00$

$\$ 3,850.00$

$\$ 9,625.00$

$\$ 385,825.44$

$\$ 3,080.00$

$\$ 3,080.00$

$\$ 3,850.00$

$\$ 30,030.00$

$\$ 5,775.00$

$\$ 105,875.00$

$\$ 38,500.00$

$\$ 0.00$

$\$ 3,850.00$

$\$ 1,925.00$

$\$ 1,925.00$

$\$ 1,925.00$

$\$ 1,925.00$

$\$ 1,925.00$

$\$ 577,500.00$

$\$ 42,900.00$

$\$ 1,925.00$

$\$ 1,925.00$

$\$ 19,250.00$

$\$ 9,625.00$

$\$ 1,925.00$

$\$ 1,925.00$

$\$ 1,925.00$

$\$ 381,363.84$

$\$ 3,850.00$

Ultra readout board prototype

$\$ 1,008,661.63$

Table 20: Estimated cost of the HFT components. The total cost for the hardware components is $\$ 3.75 \mathrm{M}$. This does not include engineering labor but it does include approximately $\$ 1 M$ in manufacturing labor to be spent in the Mechanical shops at BNL and LBL. 
The estimated costs for the HFT components and hardware are shown in Table 20. The cost table includes only purchases and manufacturing expenses. The total hardware cost is $3.75 \mathrm{M} \$$; this includes $\sim 1 \mathrm{M} \$$ manufacturing labor but does not include the engineering labor or the contributed labor.

The electronic costs shown in Table 20 are primarily for procurements from outside vendors while mechanical fabrication costs are primarily for LBNL and BNL shops. All costs shown include overhead multipliers and contingency multipliers. The contingency multiplier for the detector silicon is 1.5 , and for all other items the multiplier is 1.75 . The cost for detector silicon and ladders reflect the plan to make 2 copies of the prototype detector (with MimoSTAR IV chips) and 4 copies of the final HFT detector (with UltraSTAR chips). The pricing of the readout electronics includes $100 \%$ spares.

The MS-Project files yield a rolled-up labor summary that consumes 13.5 FTEs of engineering labor over the life of the project, 7.5 FTEs of technical labor, and 3 FTEs of management and management support. The labor costed to the project is $3 \mathrm{M} \$$, and the re-directed labor required is estimated to cost $1 \mathrm{M} \$$ at BNL and $2 \mathrm{M} \$$ at LBL. The activities at BNL include, for example, technicians to install the support cones and beam pipe. The cost for labor at BNL and LBL includes $75 \%$ contingency on wages.

The total estimated cost of the project is $7 \mathrm{M}$ for $\mathrm{R} \& \mathrm{D}$ and construction activities. The re-directed labor adds an additional $3 \mathrm{M} \$$ to the total project cost.

Studying the profile of funds for the HFT project is the next step. Currently, the profile of funds shown in the Micro-Soft-Project cost model is not a perfect match to the availability of funds. Refining this cost model is homework for the future and will appear in our Conceptual Design Report. Our goal is to match the profile of funds, and the completion date for the project, which was proposed by Brookhaven National Laboratory in the "Mid-Term Strategic Plan for the Relativistic Heavy Ion Collider"136. See Table 21.

\begin{tabular}{|c|c|c|c|}
\hline FY 2007 & FY 2008 & FY 2009 & FY 2010 \\
\hline $1 \mathrm{M}$ & $0.8 \mathrm{M}+0.3 \mathrm{M}$ & $2.5 \mathrm{M}$ & $2.5 \mathrm{M}$ \\
\hline R\&D & R\&D + PED & Construction & Construction \\
\hline
\end{tabular}

Table 21: Proposed Funding Profile for the HFT that appears in the BNL Mid Term Plan ${ }^{136}$

The HFT project was recently reviewed by the Technical Advisory Committee for RHIC detector upgrades. A copy of the cost and schedule talk that was presented to the committee is attached in "Appendix V - Cost and Schedule Presentation" and the report of the committee is attached in "Appendix VI - Report of the Technical Committee". 


\section{Summary}

Probing charm quark flow and thermal equilibration at RHIC may prove to be the final step towards the discovery of a Quark Gluon Plasma. Furthermore, measuring the energy lost by high transverse momentum heavy $(\mathrm{c}, \mathrm{b})$ quarks while traversing the medium will help disentangle between energy loss scenarios in cold nuclear matter and in partonic matter. The HFT is designed to tackle both tasks by precisely measuring open charm hadron yields, spectra and elliptic flow $\left(\mathrm{v}_{2}\right)$ as well as tagging the electrons produced by high transverse momentum beauty hadrons. The design requirements are fulfilled by having two thin ( $\leq 0.28 \%$ radiation length) layers of Active Pixel Sensors (APS) with a resolution of $10 \mu \mathrm{m}$ at the front surface of the detector. APS technology is the only option that fits these requirements without compromising the efficiency or the readout speed. Indeed, an APS can be thinned down to $50 \mu \mathrm{m}$ and their low power consumption allows us to use air-cooling. The mechanical support will be carefully designed so that the detector can be easily retracted. This feature allows the detector to be externally aligned, repaired and upgraded. By combining cutting edge sensor and readout technologies with a flexible and robust mechanical design, the HFT will provide the high precision data on heavy flavor hadrons that are crucial to understand the nature of the medium formed in $\mathrm{Au}+\mathrm{Au}$ collisions at RHIC. 


\section{Appendix I - MIMOSTAR II Specifications}

\begin{tabular}{|l|l|}
\hline Technology & AMS 0.35 opto \\
\hline Metal layers & 4 \\
\hline Epitaxial effective thickness & $\sim 12 \mu \mathrm{m}$ \\
\hline Diode size & $\begin{array}{l}1.7 \mu \mathrm{m} \times 1.7 \mu \mathrm{m} \\
2.4 \mu \mathrm{m} \times 2.4 \mu \mathrm{m}\end{array}$ \\
\hline Diode pitch & $30 \mu \mathrm{m}$ \\
\hline Signal (5 GeV electrons) & $700-800 \mathrm{e}^{-}$ \\
\hline Noise & $10 \mathrm{e}^{-}$ \\
\hline Pixels & $128 \times 128$ \\
\hline Control & $\mathrm{JTAG}$ \\
\hline Readout rate & $2 \mathrm{MHz}$ and $10 \mathrm{MHz}$ \\
\hline Operational temperature & $<40 \mathrm{C}$ \\
\hline
\end{tabular}




\section{Appendix II - MIMOSTAR II Users Guide}

This page intentionally left blank. See the next page. 


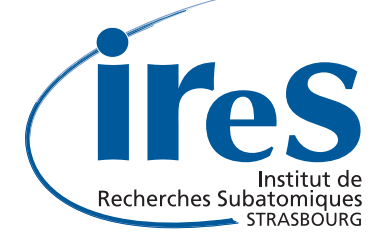

\section{MimoStar2 User Manual}

MimoStar2 User Manual

C. Colledani, W. Dulinski, H. Himmi, Ch. Hu, I.Valin Institut de Recherches Subatomiques IN2P3-CNRS / ULP Strasbourg - France 


\begin{tabular}{|l|l|l|}
\hline Document history \\
\hline Version & Date & Description \\
\hline 1.1 & January 2006 & Bias synthetic block diagram added \\
\hline 1.0 & August 2005 & MimoStar2 First Version \\
\hline 0.0 & & Based on MimoStar1 Version \\
\hline
\end{tabular}

\begin{tabular}{|l|l|l|}
\hline \multicolumn{2}{|l|}{ MimoStar chip } \\
\hline Version & Date & Description \\
\hline 2 & Submitted:June 05 & AMS 035 Version \\
\hline 1 & Submitted July 04 & TSMC 025 Version \\
\hline
\end{tabular}




\section{$\operatorname{Mimo\star 2}$}

1 Introduction

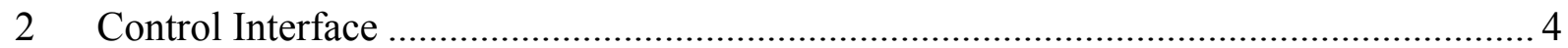

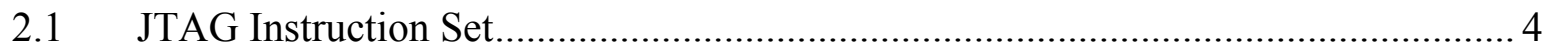

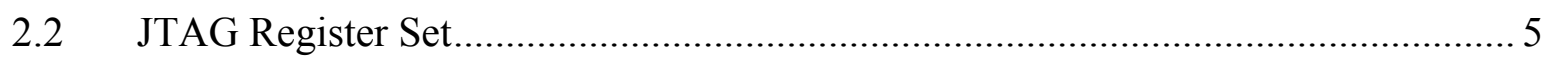

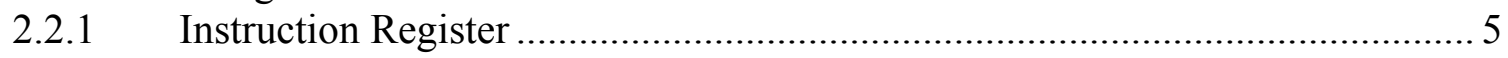

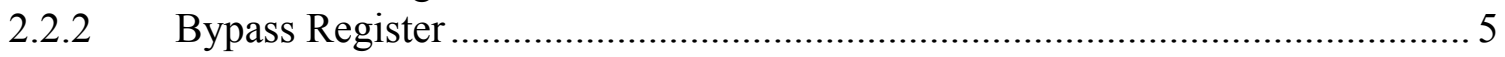

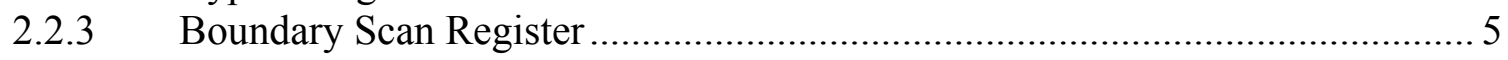

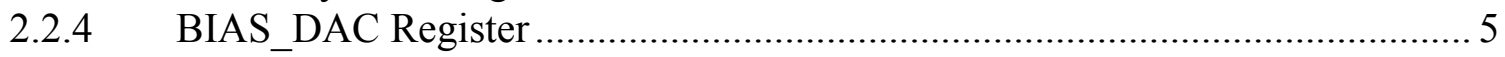

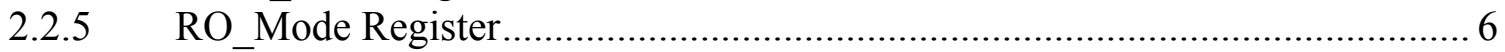

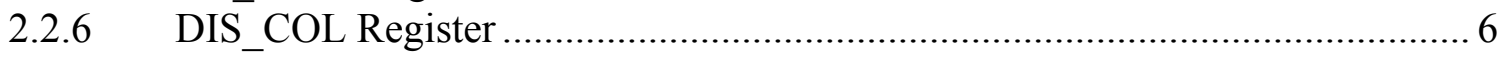

2.2.7 DEV_ID Register ............................................................................. 6

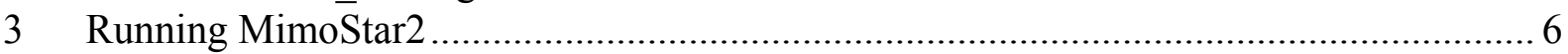

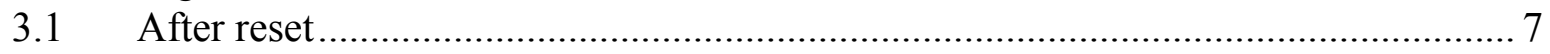

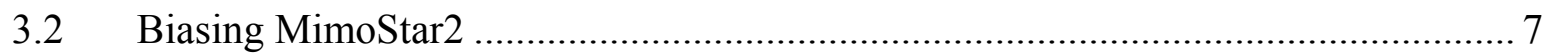

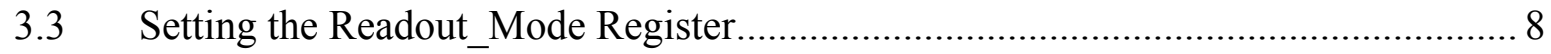

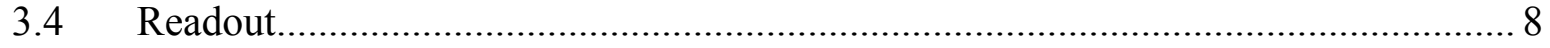

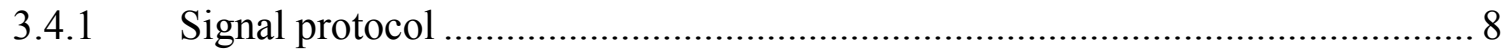

3.4.2 Successive frames and resynchronisation ................................................ 8

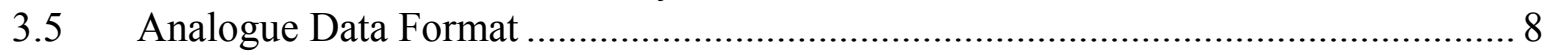

3.5.1 Normal data format ........................................................................... 8

3.5.1.1 Parallel Mode ................................................................................... 9

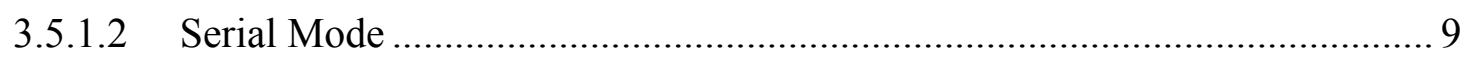

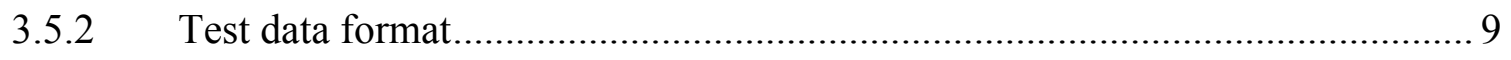

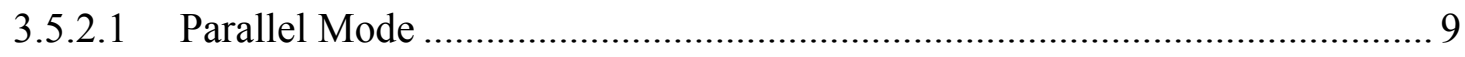

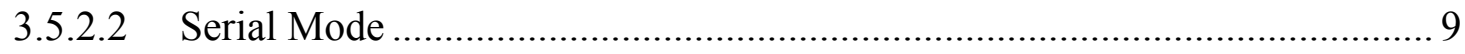

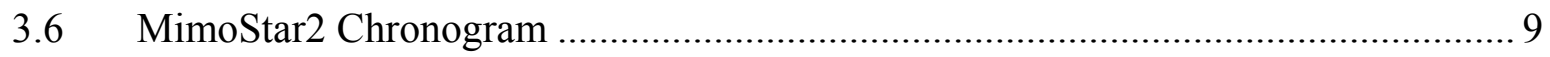

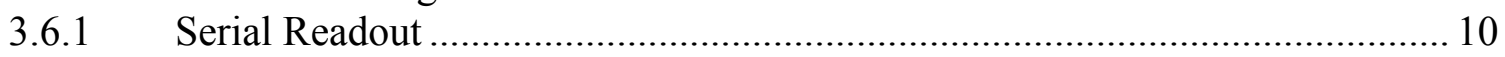

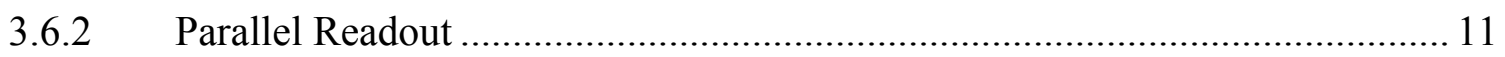

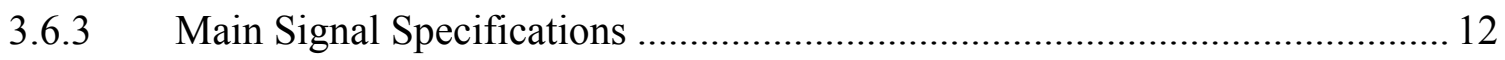

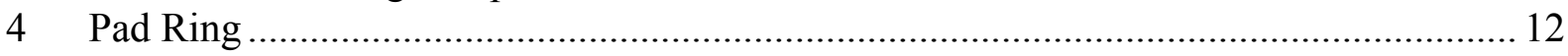

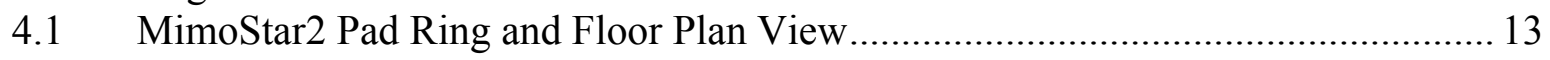

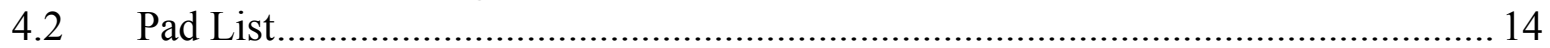




\section{$\operatorname{Mimo\star 2}$}

\section{Introduction}

Mimostar2, an enhanced version of MimoStar1, has been designed in C35B4O1, the AMS $0.35 \mu \mathrm{m}$ opto process. Like MimoStar1, it is a Monolithic Active Pixel Sensor prototype dedicated to vertex particle tracking in a future update of the STAR vertex detector. The matrix is composed by $128 \times 128$ pixels of $30 \mu \mathrm{m}$ pitch and based on self biased diode architectures. It is organised in 2 matrices, or subframes, of 128 lines x 64 columns, accessed in parallel during the readout. Each matrix contains a different pixel architecture, a so called "standard pixel" already designed and tested and a new structure which should meet the radiation tolerance and the low leakage current requirements. Actually Mimostar2 is a downsized prototype which emulates the final circuit. This one is foreseen with 640 x 640 pixels organised in 10 subframes of 640 lines x 64 columns.

The addressing of each subframe is sequential and starts from the upper left pixel up to the lower right pixel. Analogue data are extracted via a selectable set of analogue buffers. One can choose between the parallel outputs or the serial output.

- Parallel output

Two types of output voltage buffers are provided; unipolar and differential Each subframe has its dedicated buffer running at a readout speed from 5 up to $10 \mathrm{MHz}$.

- Serial output

The serialised, multiplexed, data of the 2 pixel subframes are driven out via a differential current output buffer. Its readout speed should be from 50 up to $100 \mathrm{MHz}$.

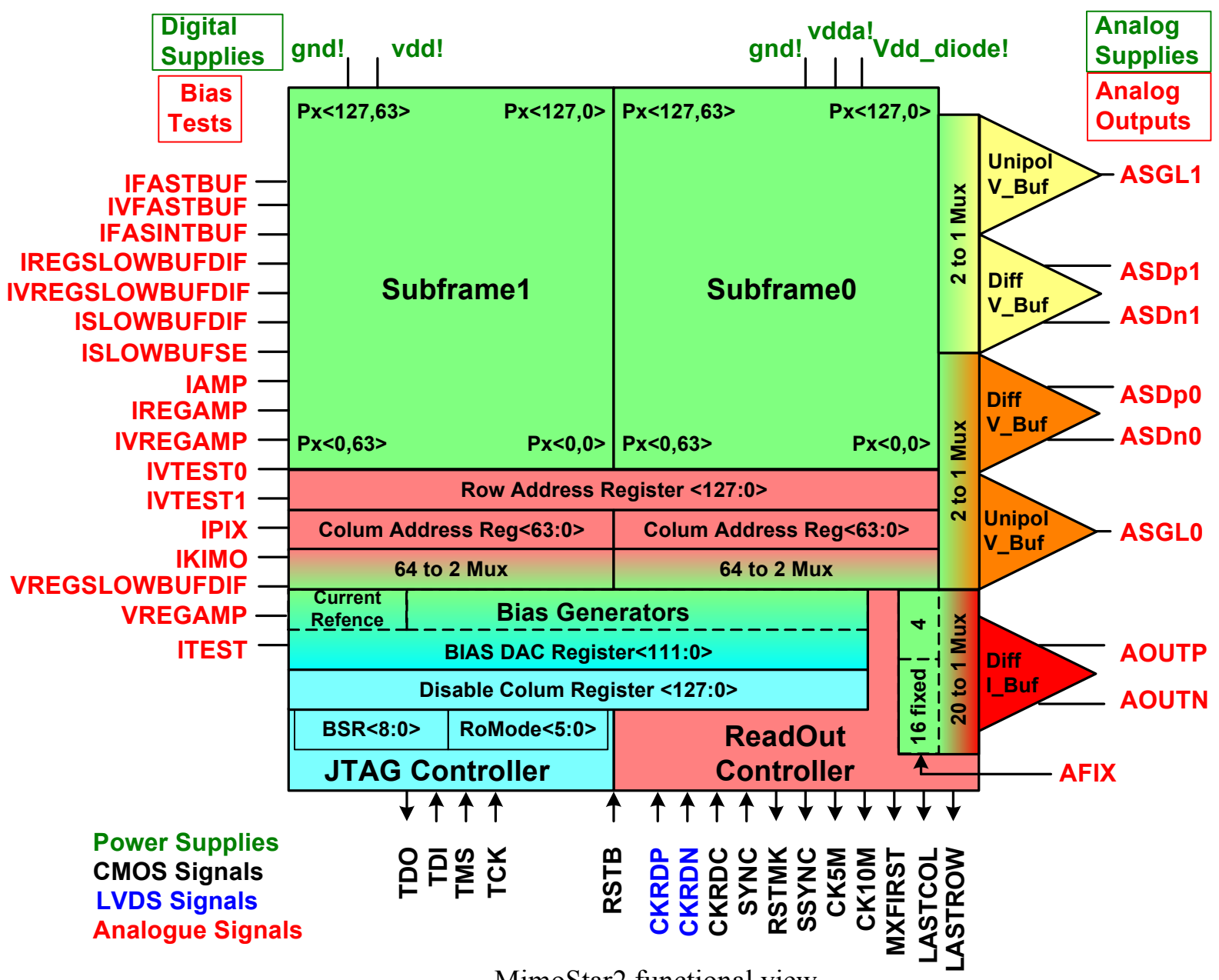

MimoStar2 functional view

Does not correspond to the floorplan; neither for the core, neither for the pad ring 


\section{$\operatorname{Mimo\star 2}$}

MimoStar2 is very simple to operate:

- Power On Reset or Reset on RSTB pad

- Setup of the chip

It is performed with programmable registers accessed via an embedded slow control interface. It consists to:

- Load the DACs which bias the analogue blocks

- Select the readout clock source among the LVDS or the CMOS input

- Select the serial or the parallel readout

- For parallel readout, select differential or single ended analogue output buffer

- The default setup after power on reset is

- CMOS input readout clock

- $10 \mathrm{MHz}$ readout clock

- Parallel differential output buffer

- Readout of the chip

- The readout starts when the input "SYNC" token signal is sampled by the readout clock. It happens at the first rising edge of the "10 MHz equivalent" clock which follows the SYNC falling edge.

- After a latency of 4 "10 MHz equivalent" clock cycles, the analogue signals appear on the selected output(s) buffer(s)

- Digital maker outputs are available for the control of the readout process

- Pixels are sequentially read out in a specific order explained later in the document

- Successive pixel frames are read until the readout clock is stopped

A frame resynchronisation can be performed at any time by setting up the "SYNC" token again.

\section{Control Interface}

The control interface of MimoStar2 complies with the Boundary Scan, JTAG, IEEE 1149.1 Rev 1999 standard. It allows the access to the internal registers of the chip like the bias register and the readout mode selection register.

On Power-On-Reset, an internal reset for the control interface is generated. The finite state machine of the Test Access Port (TAP) of the controller enters in the Test-Logic-Reset state and the ID register is selected.

\section{Remarks on the Differential Current Output Buffers:}

MimoStar2 has been designed in order to be fully adjustable via the control interface. Nevertheless the AFIX voltage level still needs to be fixed via a pad (see pad listing table).

\subsection{JTAG Instruction Set}

The Instruction Register of the JTAG controller is loaded with the code of the desired operation to perform or with the code of the desired data register to access.

\begin{tabular}{|c|c|c|c|}
\hline Instruction & 5 Bit Code $_{16}$ & Selected Register & Notes \\
\hline EXTEST & 01 & BSR & JTAG mandatory instruction \\
\hline HIGHZ & 02 & BYPASS & JTAG optional instruction \\
\hline INTEST & 03 & BSR & JTAG optional instruction \\
\hline CLAMP & 04 & BYPASS & JTAG optional instruction \\
\hline SAMPLE_PRELOAD & 05 & BSR & JTAG mandatory instruction \\
\hline BIAS GEN & 10 & BIAS register & User instruction \\
\hline DIS COL & 11 & Disable Columns & User instruction \\
\hline ID_CODE & 12 & $(1)$ & Reserved - JTAG optional instruction \\
\hline NU1 & 13 & $(1)$ & Reserved, Not Used \\
\hline NU2 & 14 & $(1)$ & Reserved, Not Used \\
\hline NU3 & 15 & $(1)$ & Reserved, Not Used \\
\hline NU4 & 16 & $(1)$ & Reserved, Not Used \\
\hline RO_MODE & 17 & Read Out Mode & User instruction \\
\hline BYPASS & $1 \mathrm{~F}$ & BYPASS & JTAG mandatory instruction \\
\hline
\end{tabular}

(1) Instruction codes implemented but not the corresponding registers. To be fixed in the next version. 


\section{$\operatorname{Mimo\star 2}$}

\subsection{JTAG Register Set}

JTAG registers are implemented with a Capture/Shift register and an Update register. JTAG standard imposes that the last significant bit of a register is downloaded/shifted first.

\begin{tabular}{|l||c|l||l||}
\hline \hline Register Name & Size & Access & Notes \\
\hline INSTRUCTION REG & 5 & R/W & Instruction Register \\
\hline BYPASS & 1 & R Only & \\
\hline BSR & 10 & R/W & \\
\hline BIAS_GEN & 112 & R/W & Previous value shifted out during write \\
\hline RO_MODE & 6 & R/W & Previous value shifted out during write \\
\hline DIS_COL & 128 & R/W & \\
\hline ID_REG, NU1, NU2, NU3, NU4 & 0 & - & Not implemented. For future use \\
\hline
\end{tabular}

\subsubsection{Instruction Register}

The Instruction register is a part of the Test Access Port Controller defined by the IEEE 1149.1 standard. The Instruction register of MimoStar2 is 5 bits long. On reset, it is set with the ID_CODE instruction. When it is read the 2 last significant bits are set with the markers specified by the standard, the remaining bits contain the current instruction.

\begin{tabular}{|l|l|l|l|l|}
\hline $\mathbf{X}$ & $\mathbf{X}$ & $\mathbf{X}$ & $\mathbf{1}$ & $\mathbf{0}$ \\
\hline
\end{tabular}

\subsubsection{Bypass Register}

The Bypass register consists of a single bit scan register. It is selected when its code is loaded in the Instruction register, during some actions on the BSR and when the Instruction register contains an undefined instruction.

\subsubsection{Boundary Scan Register}

The Boundary Scan Register, according with the JTAG instructions, tests and set the IO pads. The MimoStar2 BSR is 9 bits long and allows the test of the following input and output pads

\begin{tabular}{|l||l||l|l|l||}
\hline Bit \# & Corresponding Pad & Type & Signal & Notes \\
\hline \hline 9 & LVDS CkRdP/CkRdN & Inputs & CkRd & Resulting CMOS signal after LVDS Receiver \\
\hline \hline 8 & CMOS CkRd & Inputs & CkRd & \\
\hline \hline 7 & CMOS Sync & Inputs & Sync & \\
\hline \hline 6 & SSync & Output & SSync & \\
\hline \hline 5 & Ck5M & Output & Ck5M & \\
\hline 4 & Ck10M & Output & Ck10M & \\
\hline 3 & RstMk & Output & RstMk & \\
\hline 2 & LastRow & Output & LastRow & \\
\hline 1 & LastCol & Output & LastCol & \\
\hline 0 & MxFirst & Output & MxFirst & \\
\hline \hline
\end{tabular}

\subsubsection{BIAS_DAC Register}

The BIAS_DAC register is 112 bits large; it sets simultaneously the 14 DAC registers.

As show bellow these 8-bit DACs set voltage and current bias.

After reset the register is set to 0 , a value which fixes the minimum power consumption of the circuit.

The current values of the DACs are read while the new values are downloaded during the access to the register.

An image of the value of each DAC can be measured on its corresponding test pad.

\begin{tabular}{|l|l|l|l||l||}
\hline $\begin{array}{l}\text { Bit } \\
\text { range }\end{array}$ & DAC \# & $\begin{array}{l}\text { DAC Internal } \\
\text { Name }\end{array}$ & DAC purpose & $\begin{array}{l}\text { Corresponding } \\
\text { Test Pad }\end{array}$ \\
\hline $111-104$ & DAC13 & IKIMO & External circuit monitoring & IKIMO \\
\hline $105-96$ & DAC12 & I4PIX & Pixel source follower bias & IPIX \\
\hline \hline $95-88$ & DAC11 & V4TEST1 & Test Level, emulates a pixel output & IVTEST1 \\
\hline \hline $87-80$ & DAC10 & V4TEST0 & IDEM & IVTEST0 \\
\hline $79-72$ & DAC9 & V4REG1 & Voltage regulator voltage bias of the & IVREGAMP \\
\hline
\end{tabular}




\section{Mimo 2}

\begin{tabular}{|c|c|c|c|c|}
\hline & & & column amplifier (G3 \&5) & \\
\hline $71-64$ & DAC8 & I4REG1 & Idem & IREGAMP \\
\hline 63- 56 & DAC7 & I4AMP & Bias of the group of column amplifiers & IAMP \\
\hline $55-48$ & DAC6 & I4BUF10SE & $\begin{array}{l}\text { Bias of the single ended parallel voltage } \\
\text { Output Buffer }\end{array}$ & ISLOWBUFSE \\
\hline $47-40$ & DAC5 & I4BUF10DIF & $\begin{array}{l}\text { Bias of the differential parallel voltage } \\
\text { Output Buffer }\end{array}$ & ISLOWBUFDIF \\
\hline $39-32$ & DAC4 & V4REG2 & $\begin{array}{l}\text { Voltage regulator bias of the differential } \\
\text { parallel Output Buffer }\end{array}$ & $\begin{array}{l}\text { IVREGSLOWBUFDIF } \\
\text { F }\end{array}$ \\
\hline $31-24$ & DAC3 & I4REG2 & Idem & IREGSLOWBUFDIFF \\
\hline $23-16$ & DAC2 & I4FASTINTBUF & Bias of the fast Intermediate Buffer & IFASTINTBUF \\
\hline $15-8$ & DAC1 & V4FASTBUF & $\begin{array}{l}\text { Bias of the differential current Output } \\
\text { Buffer }\end{array}$ & IVFASTBUF \\
\hline $7-0$ & DAC0 & I4FASTBUF & Idem & IFASTBUF \\
\hline
\end{tabular}

\subsubsection{RO_Mode Register}

The RO_Mode register is 6 bits large; it allows the user to select the type of readout for the chip.

- Test mode versus normal mode

- Parallel analogue outputs versus serial output

- Amplification gain of 3 versus 5 for the serial analogue output buffer

\begin{tabular}{|c|c|c|c|c|}
\hline Bit \# & Bit Name & Purpose & & Default value \\
\hline 5 & SelLVDS & $\begin{array}{l}\text { Select LVDS versus CMOS input readout clock } \\
\text { pad }\end{array}$ & 0 & CMOS input \\
\hline 4 & SelMux & $\begin{array}{l}\text { On MxFirst output, select MuxFirst signal or } \\
\text { First Pixel of First Frame signal }\end{array}$ & 1 & $\begin{array}{l}\text { MuxFirst Signal, active } \\
\text { See } \S 3.4 \text { Readout }\end{array}$ \\
\hline 3 & EnaGain3 & $\begin{array}{l}\text { Select gain } 3 \text { for the serial differential output } \\
\text { buffer }\end{array}$ & 0 & Gain 5 \\
\hline 2 & Ena10MHz & $\begin{array}{l}\text { Select between the parallel readout or the serial } \\
\text { readout }\end{array}$ & 1 & $\begin{array}{l}10 \mathrm{MHz} \text { Clock selected } \\
\rightarrow \text { Parallel analogue outputs }\end{array}$ \\
\hline 1 & SelPSgl & $\begin{array}{l}\text { Select between differential or single ended } \\
\text { parallel analogue output }\end{array}$ & 0 & $\begin{array}{l}\text { Differential voltage output } \\
\text { buffer }\end{array}$ \\
\hline 0 & EnaTstCol & $\begin{array}{l}\text { Test Mode: Select the } 2 \text { Test Levels, IVTEST1 } \\
\text { and IVTEST0, which emulate a pixel output }\end{array}$ & 0 & Normal mode \\
\hline
\end{tabular}

\subsubsection{DIS_COL Register}

The DIS_COL register is 128 bit wide. The purpose of this register is to disable the column current sources if a short circuit is suspected on a specific column. During the readout, even if a current source is disabled the corresponding column is selected, i.e. no columns are skipped. Obviously, the signal of the corresponding pixel has not signification.

The default value of the DIS_COL register is 0 ; it means that all current sources can be activated by the readout logic. Setting a bit to 1 disables the corresponding current source. In MimoStarl, the column $<127>$ is on the left hand side while column $<0>$ is on the right hand side. The organisation of the chip in 2 subframes of 64 columns has no matter to do with the DIS_COL register.

\begin{tabular}{|lr|}
\hline $127($ Msb) & 0 (Lsb) \\
\hline \hline DisCol $<127>$ & DisCol $<0>$ \\
\hline
\end{tabular}

\subsubsection{DEV_ID Register}

The Device Identification register is not implemented yet. When selected by the ID_CODE instruction or after a reset no real data are shifted, a 0 value takes place on TDO, the JTAG serial output of the chip.

\section{Running MimoStar2}

The following steps describe how to operate Mimostar2 


\section{$\operatorname{Mimo\star 2}$}

\subsection{After reset}

On RSTB active low signal:

- All BIAS registers are set to the default value, i.e. 0

- DIS_COL is set to 0, i.e. all columns are selected

- RO_Mode is set to the binary value 10100 (see default setting)

- JTAG state machine is in the Test-Logic-Reset state

- JTAG ID_CODE instruction is selected

Then the bias register has to be loaded.

The same for the RO_MODE and DIS_COL registers if the running conditions differ from defaults.

Finally the readout can be performed either in normal mode or in test mode.

\subsection{Biasing MimoStar2}

The BIAS_DAC register has to be loaded before operating MimoStar2.

The 14 DACs constituting this register are built with the same 8 bits DAC current generator which has a $1 \mu \mathrm{A}$ resolution. Specific interfaces like current mirror for current sourcing or sinking and resistors for voltages, customise each bias output. The following table shows the downloaded codes which set the nominal bias.

\begin{tabular}{|c|c|c|c|c|c|c|c|}
\hline \multirow{2}{*}{$\begin{array}{l}\text { Internal } \\
\text { DAC } \\
\text { Name }\end{array}$} & \multicolumn{3}{|c|}{ Simulation } & \multirow{2}{*}{$\begin{array}{l}\text { Resol } \\
\text { ution }\end{array}$} & \multirow[t]{2}{*}{ Range } & \multicolumn{2}{|c|}{ Experimental } \\
\hline & $\begin{array}{l}\text { Code }_{16}- \\
\text { Code }_{10}\end{array}$ & \begin{tabular}{|lr|}
\multicolumn{2}{|l|}{ DacInterna } \\
I & current- \\
$\mu \mathrm{A}$ & \\
\end{tabular} & $\begin{array}{l}\text { Output } \\
\text { value }\end{array}$ & & & \begin{tabular}{l||} 
Code $_{16}-\mid$ \\
Code $_{10}$
\end{tabular} & $\begin{array}{l}\text { Output } \\
\text { value }\end{array}$ \\
\hline IKIMO & $64-100$ & 100 & $1 \mathrm{~V}$ & $10 \mathrm{mV}$ & From 0 up to $2.55 \mathrm{~V}$ & 64-100 & $1 \mathrm{~V}$ \\
\hline I4PIX & $1 \mathrm{E}-30$ & 30 & $30 \mu \mathrm{A}$ & $1 \mu \mathrm{A}$ & From 0 up to $255 \mu \mathrm{A}$ & \begin{tabular}{|l|}
$\mathrm{E}-30$ \\
\end{tabular} & $30 \mu \mathrm{A}$ \\
\hline V4TEST1 & C3-195 & 195 & $1.95 \mathrm{~V}$ & $10 \mathrm{mV}$ & From 0 up to $2.55 \mathrm{~V}$ & C3-195 & $1.95 \mathrm{~V}$ \\
\hline V4TEST0 & B9-185 & 185 & $1.85 \mathrm{~V}$ & $10 \mathrm{mV}$ & From 0 up to $2.55 \mathrm{~V}$ & B9-185 & $1.85 \mathrm{~V}$ \\
\hline V4REG1(Note 1) & $23-35$ & 35 & $2.95 \mathrm{~V}$ & $10 \mathrm{mV}$ & From 3.3 down to $0.75 \mathrm{~V}$ & 32-50 & $2.80 \mathrm{~V}$ \\
\hline I4REG1 & $21-33$ & 33 & $33 \mu \mathrm{A}$ & $1 \mu \mathrm{A}$ & From 0 up to $255 \mu \mathrm{A}$ & $21-33$ & $33 \mu \mathrm{A}$ \\
\hline I4AMP & $64-100$ & 100 & $100 \mu \mathrm{A}$ & $1 \mu \mathrm{A}$ & From 0 up to $255 \mu \mathrm{A}$ & $64-100$ & $100 \mu \mathrm{A}$ \\
\hline I4BUF10SE & $32-50$ & 50 & $50 \mu \mathrm{A}$ & $1 \mu \mathrm{A}$ & From 0 up to $255 \mu \mathrm{A}$ & $32-50$ & $50 \mu \mathrm{A}$ \\
\hline I4BUF10DIF & $14-20$ & 20 & $20 \mu \mathrm{A}$ & $1 \mu \mathrm{A}$ & From 0 up to $255 \mu \mathrm{A}$ & $14-20$ & $20 \mu \mathrm{A}$ \\
\hline V4REG2(Note2) & $23-35$ & 35 & $2.95 \mathrm{~V}$ & $10 \mathrm{mV}$ & From 3.3 down to $0.75 \mathrm{~V}$ & $23-35$ & $2.95 \mathrm{~V}$ \\
\hline I4REG2 & $21-33$ & 33 & $33 \mu \mathrm{A}$ & $1 \mu \mathrm{A}$ & From 0 up to $255 \mu \mathrm{A}$ & $21-33$ & $33 \mu \mathrm{A}$ \\
\hline I4FASTINTBUF & $64-100$ & 100 & $100 \mu \mathrm{A}$ & $1 \mu \mathrm{A}$ & From 0 up to $255 \mu \mathrm{A}$ & $64-100$ & $100 \mu \mathrm{A}$ \\
\hline V4FASTBUF & $5 C-92$ & 92 & $0.92 \mathrm{~V}$ & $10 \mathrm{mV}$ & From 0 up to $2.55 \mathrm{~V}$ & $5 \mathrm{C}-92$ & $0.92 \mathrm{~V}$ \\
\hline I4FASTBUF & $28-40$ & 40 & $40 \mu \mathrm{A}$ & $1 \mu \mathrm{A}$ & From 0 up to $255 \mu \mathrm{A}$ & $28-40$ & $40 \mu \mathrm{A}$ \\
\hline
\end{tabular}

Note1: Vref1 = V4REG1 - 1V; Note2: Vref2 =V4REG2 - 1V

Bias synthetic block diagram

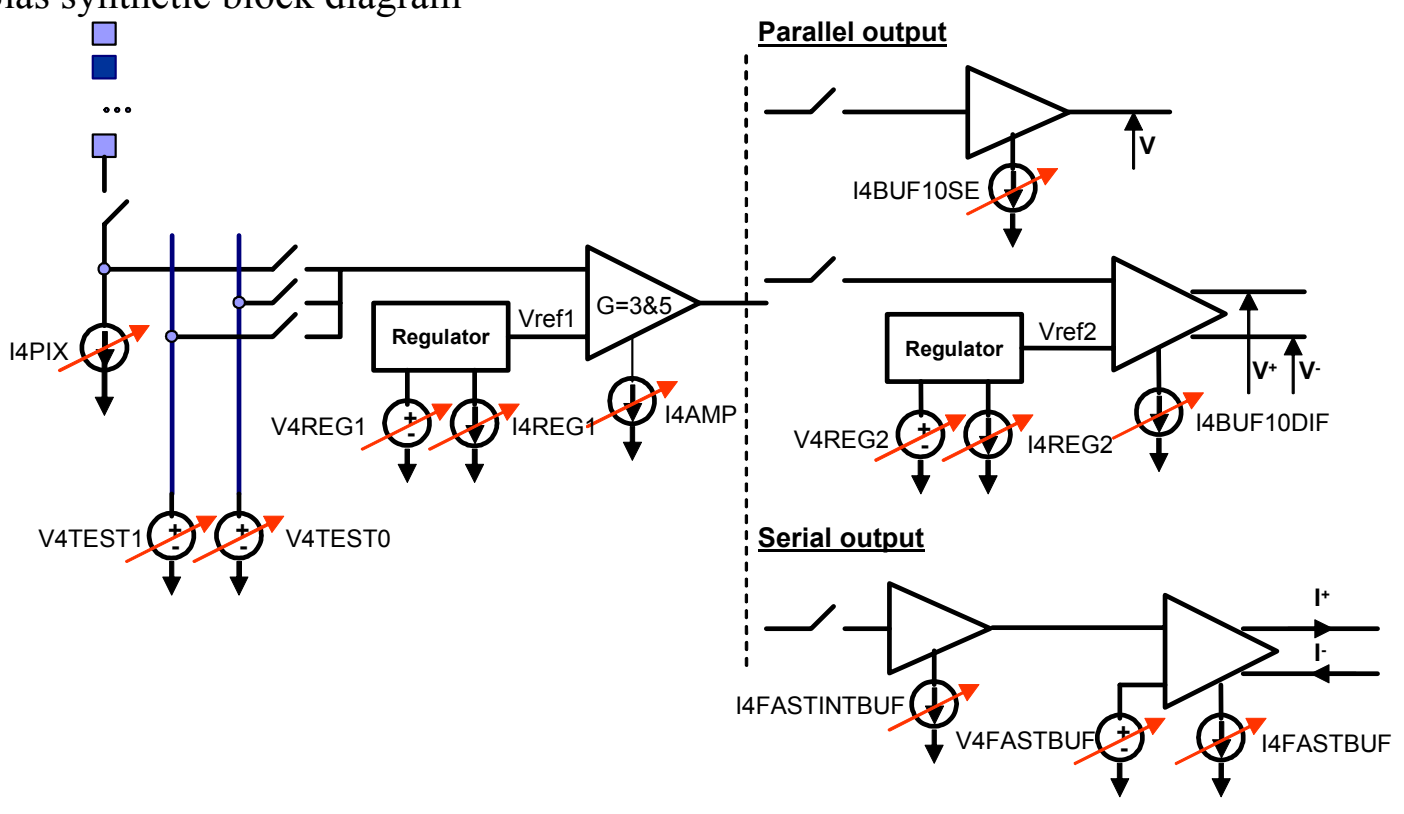




\section{$\operatorname{Mimo\star 2}$}

\subsection{Setting the Readout_Mode Register}

If the desired operating mode does not correspond to the default one, set the Readout_Mode register following the $\$ 2.2 .5$ informations.

\subsection{Readout}

\subsubsection{Signal protocol}

Ones JTAG registers have been loaded, the readout of MimoStar2 may initiate with the following signal protocol:

- The readout clock is started. This allows the CK10M output pad to generate a $10 \mathrm{MHz}$ clock. This clock follows the input clock with a $1 / 10$ ratio if the $100 \mathrm{MHz}$ is selected.

- The SYNC signal is set.

- The readout starts at the first rising edge of CK10M of after SYNC signal disappears.

- Signal markers allow the readout monitoring and the analogue data sampling:

- RstMk maker confirms the internal reset of the readout logic.

- SSync marker shows that the readout starts.

- 4 extra CK10M clock cycles, after SYNC sampling, are necessary before the first pixel analogue signal appears on the selected output(s).

- The MxFirst digital signal helps for a better sampling of the analogue output signals. The way it acts is set by the RO Mode[4] bit.

- RO_Mode $[\overline{4}]=0$ : MxFirst is active only on the first pixel oft the first frame

- RO_Mode[4] = 1: MxFirst is active on each pixel change on the parallel analogue output i.e. it is a10 $\mathrm{MHz}$ periodic signal.

Used with the $100 \mathrm{MHz}$ serial mode (see serial data format bellow), its period shows when one pixel index has been read in all the subframes $(2$ real +8 virtual $)$.

- LastCol is active when the last column of the current row is selected

- LastRow is active when the last row of the frame is selected

- $\mathrm{Ck} 5 \mathrm{MHz}$ output shows the internal clock running as long as input clock (10 or $100 \mathrm{MHz})$ is running.

\subsubsection{Successive frames and resynchronisation}

Successive pixel frames are read until the readout clock is stopped.

A frame resynchronisation can be performed at any time by setting up the "SYNC" token again.

\subsection{Analogue Data Format}

Two types of signal can be generated in serial or in parallel mode

- Normal pixel signal

- Test signal.

This gives 4 different formats of data

- Normal Serial Format

- Normal Parallel Format

- Test Parallel Format

- $\quad$ Test Serial Format

\subsubsection{Normal data format}

In order to improve the readout speed Mimostar2 is organized in subframes, i.e. 2 subframes for this current prototype and 10 for the foreseen full-size version.

During the readout, the 2 subframes of Mimostar 2 are accessed in parallel. For each subframe the addressing is done row by row, each pixel is accessed sequentially from the left side to right side. Each row contains 2 dummy pixels, and 64 active pixels. During the readout one can use the adjustable level of the 2 dummy pixels as a pattern recogniser. If the pixel coordinate format is specified as $\mathrm{Px}<\mathrm{Line}$, Column $>$, then for each subframe, the upper left pixel is $\mathrm{Px}<127,63>$ while the lower right is $\mathrm{Px}<0,0>$ and the dummy pixels of each beginning row are named Dp1 and Dp0. 


\section{$\operatorname{Mimo\star 2}$}

\subsubsection{Parallel Mode}

There is one output buffer per subframe: thus the Normal Parallel Mode data stream format for each output is:

$\mathrm{Dp} 1, \mathrm{Dp} 0, \mathrm{Px}<127,63>, \mathrm{Px}<127,62>$, . . ., $\mathrm{Px}<127,0>$

$\mathrm{Dp} 1, \mathrm{Dp} 0, \mathrm{Px}<126,63>, . \mathrm{Px}<126,62>, . . . \mathrm{Px}<126,0>$

$\cdot \cdot \cdot \cdot \cdot \cdot \cdot \cdot \cdot \cdot \cdot \cdot \cdot \cdot \cdot \cdot \cdot \cdot \cdot \cdot \cdot \cdot \cdot \cdot \cdot \cdot \cdot \cdot$

$\mathrm{Dp} 1, \mathrm{Dp} 0, \mathrm{Px}<1,63>, . \mathrm{Px}<1,62>, . . ., \mathrm{Px}<1,0>$

$\mathrm{Dp} 1, \mathrm{Dp} 0, \mathrm{Px}<0,63>, . \mathrm{Px}<0,62>, . . ., \mathrm{Px}<0,0>$

\subsubsection{Serial Mode}

The serial mode consists to read successively one pixel of each subframe and then turning back to the first subframe in order to read its next pixel. Even if Mimostar2 is a downsized prototype of 2 subframes the serial readout strategy has been maintained for 10. This implies for the serial format 8 dummy values on 10 analogue data. These dummy values are fixed via the AFIX pad.

For Mimostar2 the left hand side subframe is named Sf1 and the right hand side is Sf0 while the dummy values generated in place of the 8 non-existing subrames are named from Dv7 to Dv0.. Thus the normal data stream in serial mode has the following format:

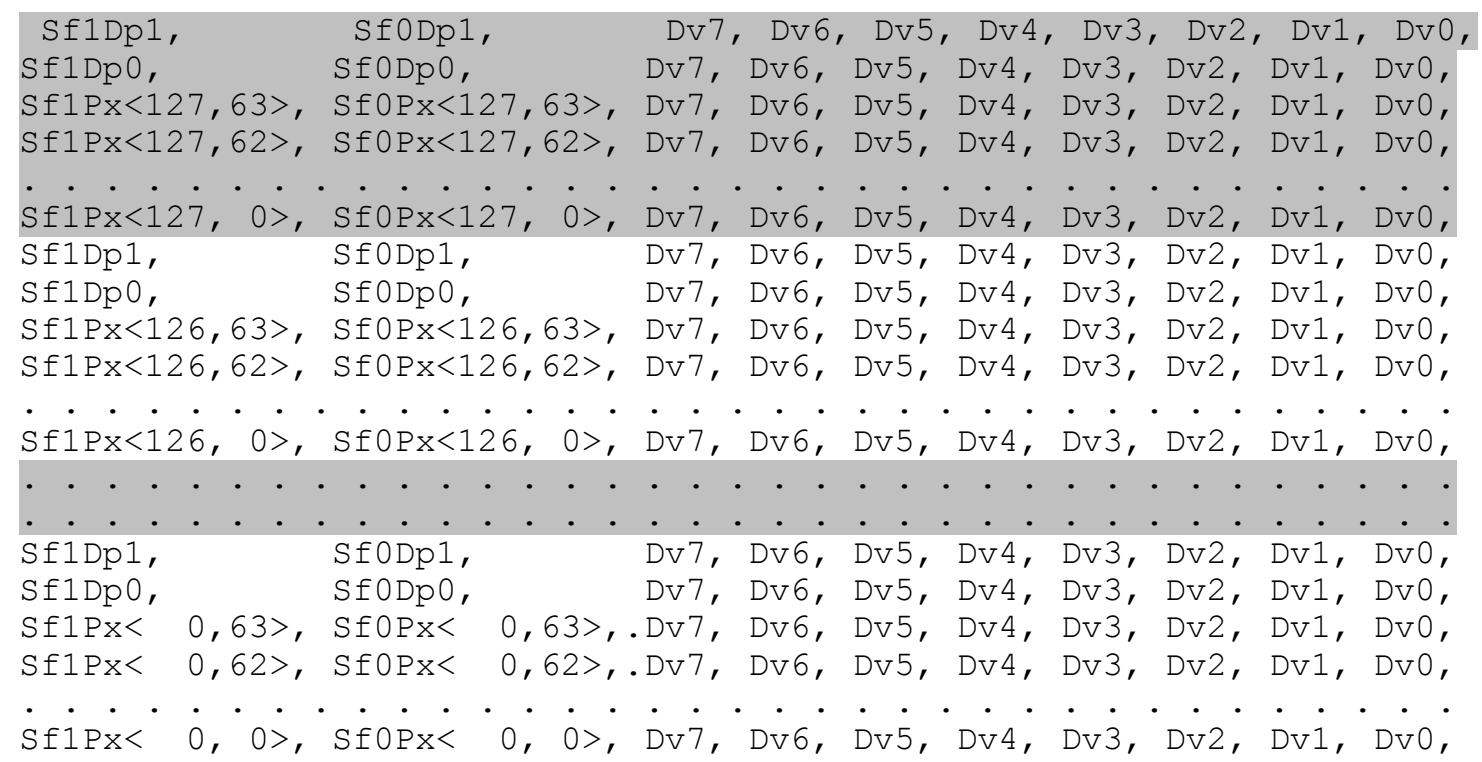

\subsubsection{Test data format}

During the test mode the pixel matrix is not connected to the multiplexing electronic. In place of it, two test levels V4TEST1 (V1), V4TEST0 (V0) are available. They emulate two pixel level outputs. Actually these levels correspond to those of Dummy Pixel 1 and Dummy Pixel 0. They are adjustable via 2 DACs. Even and odd columns are alternatively connected to one of them. This pattern allows seeing the output signal changing and emulates the readout shift from one column of pixel to the other column of pixel.

\subsubsection{Parallel Mode}

There is one output buffer per subframe: thus the Test Parallel Mode data stream format per output is:

Subframe 1: V1, V0, V0, V1, V1, V0, V0, V1 . . .

Subframe 0: v0, V1, V1, v0, V0, V1, V1, V0...

\subsubsection{Serial Mode}

Test data stream in serial mode has the following format:

Sflv1, Sfovo, Sf1vo, Sfov1, Sflvo, Sfov1, Sfov1, sfovo,

SflV1, Sf0V0, SflV0, Sf0V1, SflV0, Sf0V1, Sf0V1, Sf0V0.. .

\subsection{MimoStar2 Chronogram}

The following chronograms describe typical access to Mimostar2; Reset, JTAG download sequence and then the readout of the chip. This one starts with the initialisation phase followed by the successive row readouts as showed in the zoom. 


\section{$\operatorname{Mimo\star 2}$}

\subsubsection{Serial Readout}

Figure 1 show a typical readout in serial mode. After Reset and JTAG settings, one can see the readout oft the first pixel row readout followed by successive rows readout marked by the LastCol signal. Finally the LastRow signal active high indicates that the readout of the last row of the frame is performed.

Figure 2 zoom on the readout start. One can see that it starts after a latency of 4 CK10M cycles following the sampling of SYNC low by this clock. Mxfirst goes active and the analogue signal generated in respect with the serial format.

Figure 3 zoom on the transition between 2 consecutive rows. The 2 dummy pixels of each subframes are clearly showed.

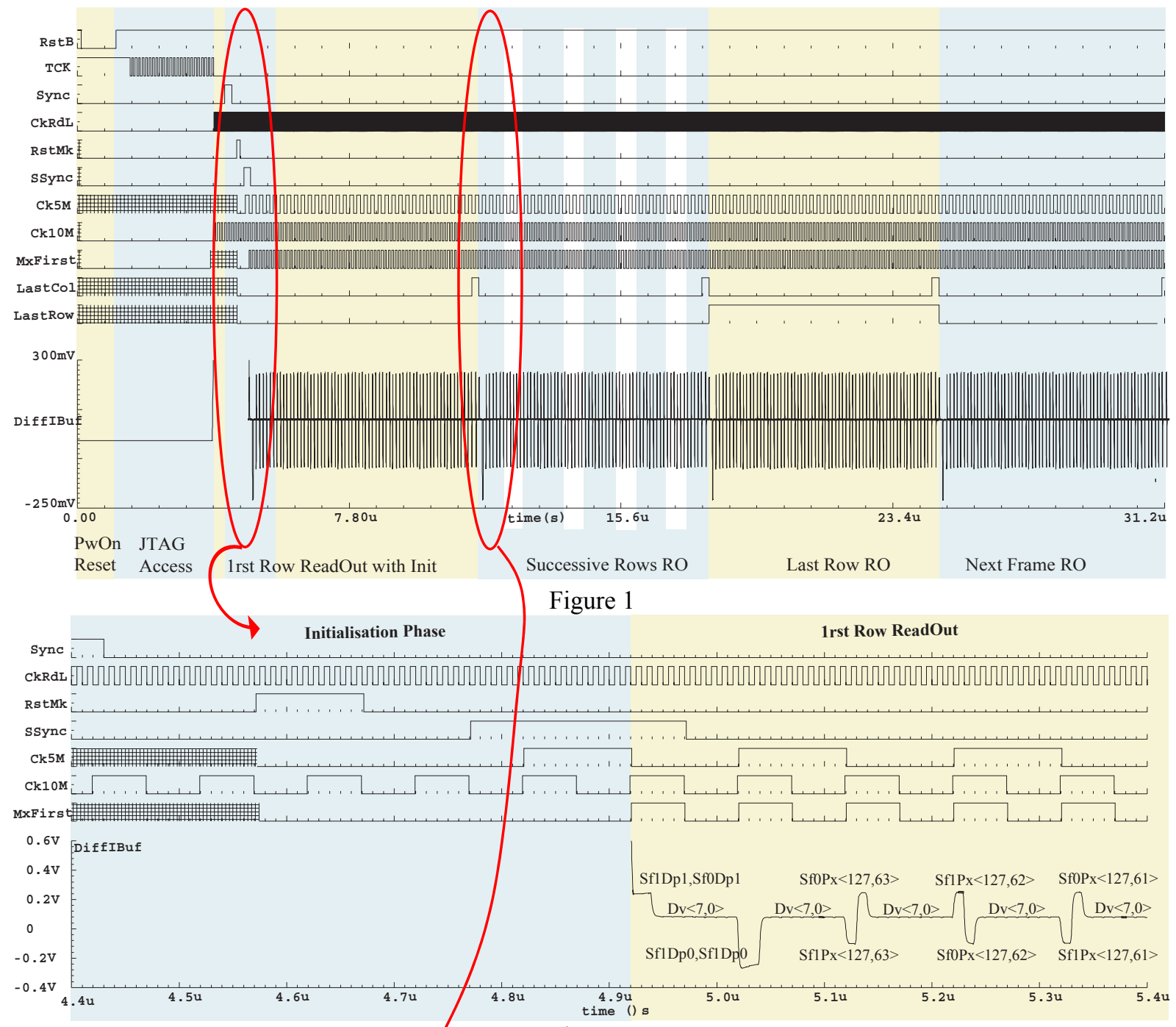

Figure 2

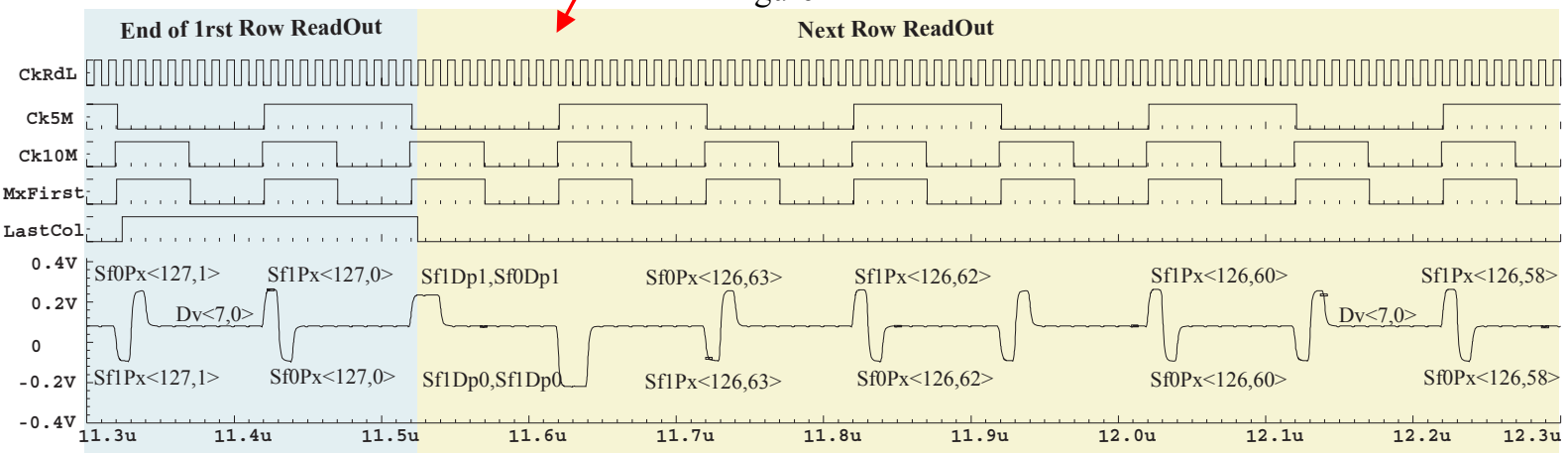

Figure 3 


\section{$\operatorname{Mimo\star 2}$}

\subsubsection{Parallel Readout}

Figure 1 show the beginning of a typical readout in parallel mode. After Reset and JTAG settings, one can see the readout oft the first pixel row readout followed the successive one indicated by the LastCol signal.. The parallel analogue outputs are showed. One can distinguish the 2 dummy pixels at the beginning of each row readout.. This is clearer in the zoom of figure 2
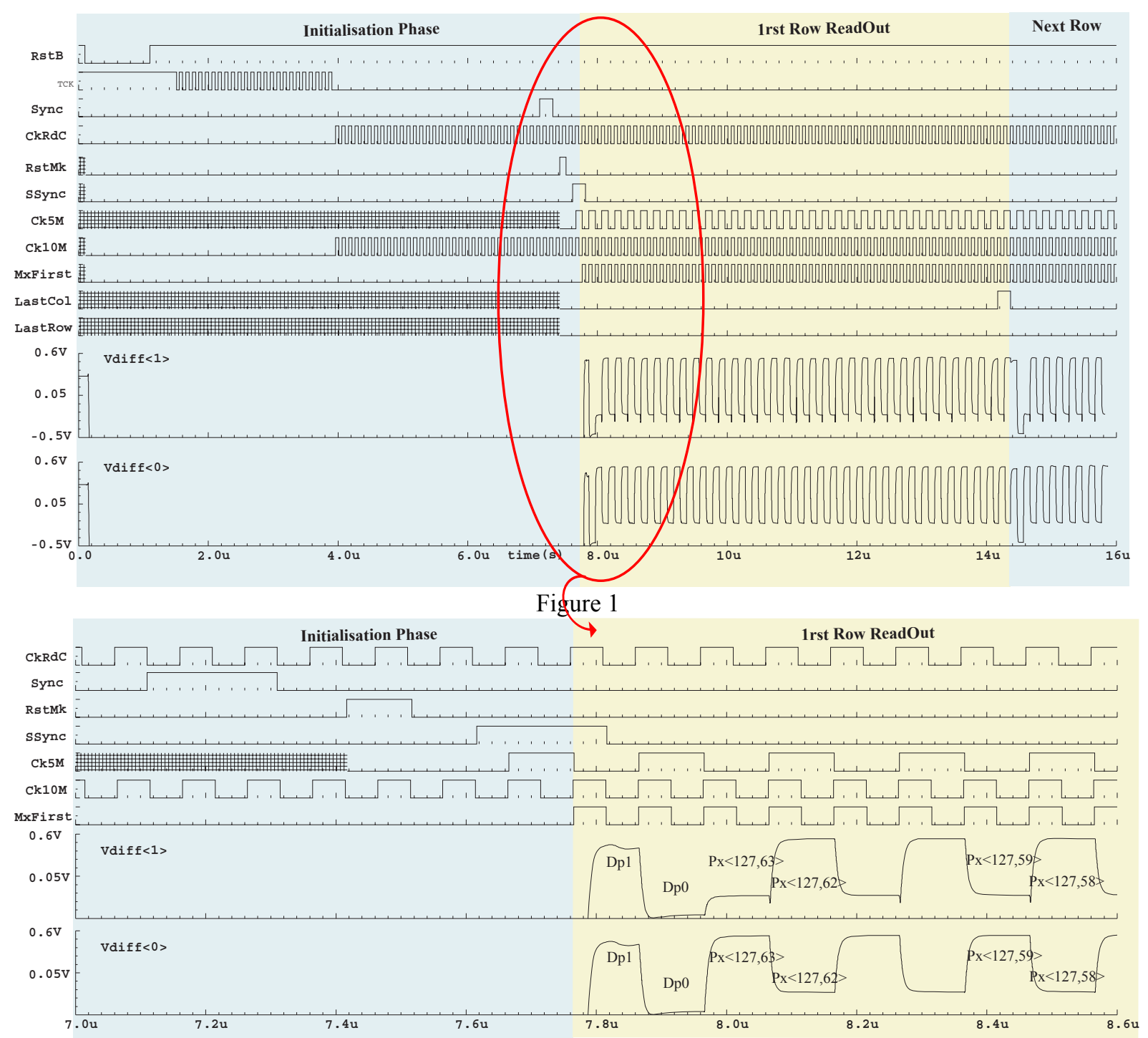

Figure 2 


\section{$\operatorname{Mimo\star 2}$}

\subsubsection{Main Signal Specifications}

\begin{tabular}{|c|c|c|c|}
\hline & Parameter & Typical Value & Notes \\
\hline INIT & RSTB Pulse Width & $>1 \mu \mathrm{S}$ & Active Low, Asynchronous Power on Reset \\
\hline \multirow{3}{*}{ JTAG } & TCK Frequency & $10 \mathrm{MHz}$ & Boundary Scan Clock \\
\hline & TMS Setup/Hold Time & $\sim 10 \mathrm{nS}$ & Boundary Scan Control Signal \\
\hline & TDI Setup/Hold Time & $\sim 10 \mathrm{nS}$ & Boundary Scan Serial Data In \\
\hline \multirow[t]{4}{*}{ READOUT } & CKRD Frequency & Up to $100 \mathrm{MHz}$ & Readout Clock LVDS signal \\
\hline & CKRD Frequency & Up to $10 \mathrm{MHz}$ & Readout Clock CMOS signal \\
\hline & CKRD Duty Cycle & $50 \%$ & \\
\hline & SYNC Setup/Hold Time & $5 \mathrm{nS}$ & $\begin{array}{l}\text { Chip Initialisation, CMOS signal. Starts after } \\
\text { falling edge on 1rst CKRD sampling } \\
\end{array}$ \\
\hline \multirow{6}{*}{$\begin{array}{l}\text { Differential } \\
\text { Current } \\
\text { Buffer (1) }\end{array}$} & Input Dynamic range & 0.7 up to $1.2 \mathrm{~V}$ & \\
\hline & Rise time & $5 \mathrm{nS}$ & \multirow{2}{*}{$\begin{array}{l}\text { @ } 10-90 \% \text {, for fully input dynamic range } \\
\text { Simulated with } Z_{\text {load }}=2 * 100 \text { Ohm and } 2 * 5 \mathrm{pF}\end{array}$} \\
\hline & Fall time & $5 \mathrm{nS}$ & \\
\hline & Bandwidth & $245 \mathrm{MHz}$ & (a) $-3 \mathrm{~dB}$ \\
\hline & Transconductance gain & $5.8 \mathrm{mS}$ & \\
\hline & Output Current Range & $-2.2 ; 2.2 \mathrm{~mA}$ & \\
\hline AFIX & Bias value & $1.5 \mathrm{~V}$ & Serial Output Buffer Dummy Data \\
\hline \multirow{4}{*}{$\begin{array}{l}\text { Differential } \\
\text { Voltage } \\
\text { Buffer }\end{array}$} & Rise time & $10 \mathrm{nS}$ & \multirow[t]{2}{*}{ @ $10-90 \%$} \\
\hline & Fall Time & $10 \mathrm{nS}$ & \\
\hline & Bandwidth & $40 \mathrm{MHz}$ & (a) $-3 \mathrm{~dB}$ \\
\hline & Capacitance load & $1 \mathrm{pF}$ & \\
\hline \multirow{3}{*}{$\begin{array}{l}\text { Unipolar } \\
\text { Buffer (2) }\end{array}$} & Rise/ fall time & $6 \mathrm{nS}$ & \multirow{2}{*}{$\begin{array}{l}\text { @ } 10-90 \% \text {, Full analogue chain simulated with } \\
\text { load capacitance of } 10 \mathrm{pF}\end{array}$} \\
\hline & Fall Time & $7 \mathrm{nS}$ & \\
\hline & Capacitance load & $10 \mathrm{pF}$ & \\
\hline
\end{tabular}

Note 1: The differential current output buffer can be modelled as an ideal current source. Its performances in terms of raising and falling times are limited by its load's time constant $\left(\mathrm{R}_{\text {load }} \mathrm{x} \mathrm{C}_{\text {load }}\right)$

Note 2: Simple source follower

\section{Pad Ring}

The pad ring of Mimlostar2 is build with

- Pads full custom designed for some of the analogue signals and power supplies

- $\quad$ Pads from the AMS library for the digital signals and power supplies

The pad ring is split in 6 functional independent parts

- CMOS JTAG and Read Out Control

- LVDS Read Out Drivers

- Analogue Core Supplies

- Read Out Analogue Outputs

- Test Structure pads, Mimostar1 independent

- Bias Test

Each part has its own supply pads. 


\section{$\operatorname{Mimo\star 2}$}

\subsection{MimoStar2 Pad Ring and Floor Plan View}

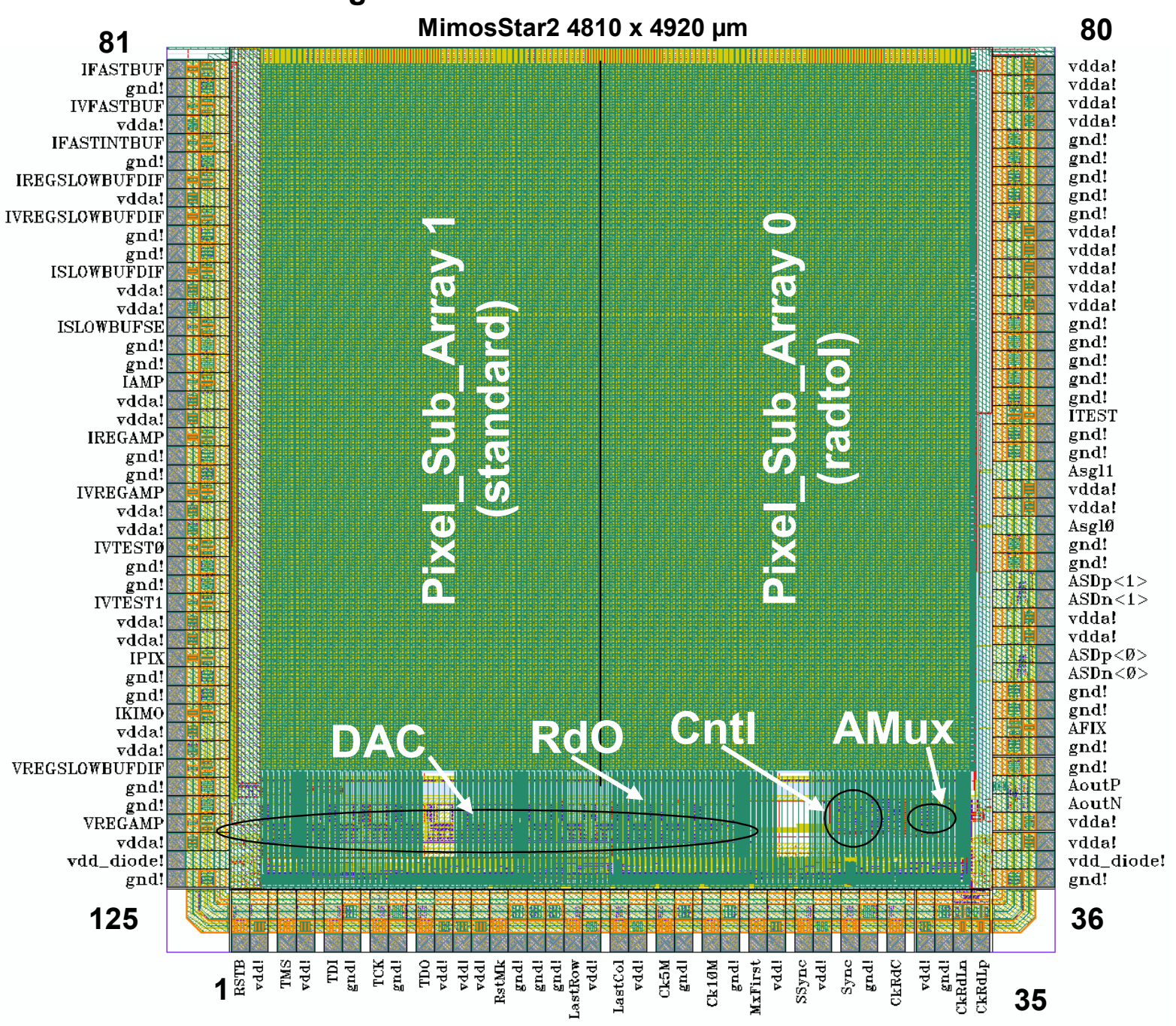

Total Pads $=125$ - Supply Pads: Gnd $=42$, Vdd $=9$, AVdd $=28$

\section{Foundry submission information}

Mimostar2 has been designed in AMS C35B4O1 CMOS $0.35 \mu \mathrm{m}$ epitaxial and opto process with 2 poly and 4 metal layers.

The Process Design Kit V3.60 has been provided by CMP

CAD tools are CADENCE DFII 5.0 with DIVA and ASSURA rules

The chip has been submitted in a Multi Chip Run via CMP the 25 June 2005 in the run \# A35C5-4. 


\section{$\operatorname{Mimo\star 2}$}

\subsection{Pad List}

The bonding of the power supply pads specified in red colour is mandatory

\begin{tabular}{|c|c|c|c|c|}
\hline \multicolumn{5}{|c|}{ Horizontal Bottom Side } \\
\hline Pad & Name & \begin{tabular}{|l|} 
Pad General Function \\
\end{tabular} & PadType & Function for the chip \\
\hline 1 & RSTB & Schmitt-Trigger Input Buffer, Pull Up & $\mid \overline{\text { ISUP }}$ & $\begin{array}{l}\text { Asynchronous Active Low } \\
\text { Reset }\end{array}$ \\
\hline 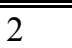 & VDD & Pad supplying the output buffers & VDD3OP & $3.3 \mathrm{~V}$ \\
\hline 3 & TMS & CMOS Input Buffer, Pull Up & ICUP & \begin{tabular}{|l} 
JTAG Control Signal \\
\end{tabular} \\
\hline 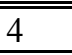 & VDD & Core logic and periphery cells supply & VDD3RP & 3.3 V periphery cells only \\
\hline 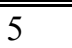 & TDI & CMOS Input Buffer, Pull Up & ICUP & JTAG Serial Data In \\
\hline 6 & GND & Pad supplying the output buffers & GND3OP & Ground \\
\hline 7 & TCK & CMOS Clock Input Buffer, 2 mA & ICCK2P & JTAG Clock \\
\hline 8 & GND & Core logic and periphery cells supply & VDD3RP & Ground periphery cells only \\
\hline 9 & TDO & \begin{tabular}{|l} 
Tri-State Output Buffer, $4 \mathrm{~mA}$ \\
\end{tabular} & BT4P & JTAG Serial Data Out \\
\hline 10 & VDD & Core logic and periphery cells supply & VDD3RP & $3.3 \mathrm{~V}$ periphery \& core \\
\hline 11 & VDD & Core logic and periphery cells supply & VDD3RP & 3.3 V periphery \& core \\
\hline 12 & VDD & Pad supplying the output buffers & VDD3OP & $3.3 \mathrm{~V}$ \\
\hline 13 & RstMk & \begin{tabular}{|l} 
Tri-State Output Buffer, $2 \mathrm{~mA}$ \\
\end{tabular} & BT2P & Readout Reset Marker \\
\hline 14 & GND & Core logic and periphery cells supply & VDD3RP & Ground periphery \& core \\
\hline 15 & GND & Core logic and periphery cells supply & VDD3RP & Ground periphery \& core \\
\hline 16 & GND & Pad supplying the output buffers & GND3OP & Ground \\
\hline 17 & LastRow & \begin{tabular}{|l} 
Tri-State Output Buffer, $2 \mathrm{~mA}$ \\
\end{tabular} & BT2P & Last Row Maker \\
\hline 18 & \begin{tabular}{|l|l} 
VDD \\
\end{tabular} & Core logic and periphery cells supply & VDD3RP & 3.3 V periphery cells only \\
\hline 19 & LastCol & Tri-State Output Buffer, $2 \mathrm{~mA}$ & BT2P & Last Column Marker \\
\hline 20 & VDD & Pad supplying the output buffers & VDD3OP & $3.3 \mathrm{~V}$ \\
\hline 21 & CK5M & Tri-State Output Buffer, $2 \mathrm{~mA}$ & BT2P & $5 \mathrm{MHz}$ Clock Out \\
\hline 22 & GND & Core logic and periphery cells supply & GND3RP & \begin{tabular}{|l} 
Ground periphery cells only \\
\end{tabular} \\
\hline 23 & CK10M & Tri-State Output Buffer, $2 \mathrm{~mA}$ & BT2P & $10 \mathrm{MHz}$ Clock Out \\
\hline 24 & GND & Pad supplying the output buffers & GND3OP & Ground \\
\hline 25 & MxFirst & Tri-State Output Buffer, $2 \mathrm{~mA}$ & BT2P & Subframes odd pixel selection \\
\hline$\overline{26}$ & \begin{tabular}{|l|l} 
VDD \\
\end{tabular} & Core logic and periphery cells supply & VDD3RP & 3.3 V periphery cells only \\
\hline 27 & SSnc & \begin{tabular}{|l} 
Tri-State Output Buffer, $2 \mathrm{~mA}$ \\
\end{tabular} & BT2P & Readout Synchro. Start Marker \\
\hline 28 & VDD & Pad supplying the output buffers & VDD3OP & $3.3 \mathrm{~V}$ \\
\hline 29 & Sync & CMOS Input Buffer & ICP & Readout Input token \\
\hline 30 & GND & Core logic and periphery cells supply & GND3RP & Ground periphery cells only \\
\hline 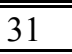 & CkRdC & CMOS Input Buffer, Pull Down & ICDP & \begin{tabular}{|l} 
Readout Clock Signal \\
\end{tabular} \\
\hline 32 & VDD & LVDS Pad Supply & AVDDALLP & 3.3V for LVDS Pad \\
\hline 33 & GND & LVDS Pad Ground & AGNDALLP & Ground for LVDS Pad \\
\hline 34 & CkRdLn & LVDS In- & Full Custom & Readout Clock Signal \\
\hline 35 & CkRdLp & LVDS In+ & Full Custom & \\
\hline
\end{tabular}




\section{$\operatorname{Mimo\star 2}$}

\begin{tabular}{|c|c|c|c|c|}
\hline \multicolumn{5}{|c|}{ Vertical Right Hand Side } \\
\hline Pad & Name & Pad General Function & PadType & Function for the chip \\
\hline 36 & GND & & AGNDALLP & Analogue Core Ground \\
\hline 37 & Vdd-Diode & Direct Pad, no protections & Full Custom & Pixel Diode Bias, 3.3V \\
\hline 38 & \begin{tabular}{|l} 
VDDA \\
\end{tabular} & Analogue Supply & AVDDALLP & Analogue Core Supply \\
\hline 39 & VDDA & Analogue Supply & AVDDALLP & Diff. Buffer Voltage Supply \\
\hline 40 & AoutN & \multirow[t]{2}{*}{ Empty pad with embedded buffer } & Full Custom & \multirow{2}{*}{$\begin{array}{l}\text { Serial Differential Current } \\
\text { Output Buffer }\end{array}$} \\
\hline 41 & AoutP & & Full Custom & \\
\hline 42 & GND & Analogue Gnd & AGNDALLP & Diff. Buffer Ground Supply \\
\hline 43 & GND & Analogue Gnd & AGNDALLP & Analogue Pad Ring Gnd \\
\hline 44 & $\overline{\mid A F I X}$ & Analog I/O pad, $0 \Omega$ serial resistor. & APRIOP & $\begin{array}{l}\text { Serial OutputBuffer Dummy } \\
\text { Data }\end{array}$ \\
\hline 45 & GND & Analogue Pad Gnd & AGNDALLP & Analogue Pad Ring Gnd \\
\hline 46 & GND & Analogue Pad Gnd & AGNDALLP & Analogue Pad Ring Gnd \\
\hline 47 & ASDn $<0>$ & \multirow[t]{2}{*}{ Empty pad with embedded buffer } & Full Custom & \multirow[t]{2}{*}{ Differential parallel output $<0>$} \\
\hline 48 & ASDp $<0>$ & & Full Custom & \\
\hline 49 & VDDA & Analogue Core Supply & AVDDALLP & Analogue Pad Ring Supply \\
\hline 50 & VDDA & Analogue Core Supply & AVDDALLP & Analogue Pad Ring Supply \\
\hline 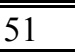 & ASDn $<1>$ & Analogue voltage Out- & Full Custom & \multirow[t]{2}{*}{ Differential parallel output $<1>$} \\
\hline$\overline{52}$ & ASDp $<1>$ & Analogue voltage OutP+ & Full Custom & \\
\hline 53 & GND & Analogue Pad Gnd & AGNDALLP & Analogue Pad Gnd \\
\hline 54 & GND & Analogue Pad Gnd & \begin{tabular}{|l} 
AGNDALLP \\
\end{tabular} & Analogue Pad Gnd \\
\hline$\overline{55}$ & Asgl0 & Empty pad with embedded buffer & Full Custom & Analogue Single Ended Output \\
\hline 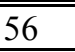 & VDDA & Analogue Core Supply & AVDDALLP & VDDA \\
\hline 57 & VDDA & Analogue Core Supply & |AVDDALLP & VDDA \\
\hline$\overline{58}$ & Asg10 & Empty pad with embedded buffer & Full Custom & Analogue Single Ended Output \\
\hline 59 & GND & Analogue Pad Gnd & AGNDALLP & Analogue Pad Gnd \\
\hline 60 & GND & Analogue Pad Gnd & AGNDALLP & Analogue Pad Gnd \\
\hline 61 & ITEST & Analog I/O pad, $0 \Omega$ serial resistor. & APRIOP & Internal Current Ref Source Test \\
\hline 62 & GND & Analogue Pad Gnd & AGNDALLP & Analogue Pad Gnd \\
\hline$\overline{63}$ & GND & Analogue Pad Gnd & AGNDALLP & Analogue Pad Gnd \\
\hline 64 & GND & Analogue Pad Gnd & AGNDALLP & Analogue Pad Gnd \\
\hline 65 & GND & Analogue Pad Gnd & AGNDALLP & Analogue Pad Gnd \\
\hline 66 & GND & Analogue Pad Gnd & AGNDALLP & Analogue Pad Gnd \\
\hline 67 & VDDA & Analogue Core Supply & AVDDALLP & VDDA \\
\hline 68 & VDDA & Analogue Core Supply & AVDDALLP & VDDA \\
\hline 69 & VDDA & Analogue Core Supply & AVDDALLP & $\begin{array}{l}\text { VDDA } \\
\end{array}$ \\
\hline 70 & VDDA & Analogue Core Supply & AVDDALLP & VDDA \\
\hline 71 & VDDA & Analogue Core Supply & |AVDDALLP & VDDA \\
\hline$\overline{72}$ & GND & Analogue Pad Gnd & AGNDALLP & Analogue Pad Gnd \\
\hline 73 & GND & Analogue Pad Gnd & AGNDALLP & Analogue Pad Gnd \\
\hline 74 & GND & Analogue Pad Gnd & $\begin{array}{l}\text { AGNDALLP } \\
\end{array}$ & Analogue Pad Gnd \\
\hline 75 & GND & Analogue Pad Gnd & AGNDALLP & Analogue Pad Gnd \\
\hline 76 & GND & Analogue Pad Gnd & AGNDALLP & Analogue Pad Gnd \\
\hline 77 & VDDA & Analogue Core Supply & AVDDALLP & VDDA \\
\hline 78 & VDDA & Analogue Core Supply & AVDDALLP & VDDA \\
\hline$\overline{79}$ & VDDA & Analogue Core Supply & AVDDALLP & $\begin{array}{l}\text { VDDA } \\
\end{array}$ \\
\hline 80 & VDDA & Analogue Core Supply & AVDDALLP & VDDA \\
\hline
\end{tabular}




\section{$\operatorname{Mimo\star 2}$}

\begin{tabular}{|c|c|c|c|c|}
\hline \multicolumn{5}{|c|}{ Vertical Left Hand Side } \\
\hline Pad & Name & Pad General Function & PadType & Function for the chip \\
\hline 81 & IFASTBUF & Analog I/O pad, $0 \Omega$ serial & APRIOP & DAC Test Purpose Only \\
\hline 82 & GND & Analogue Pad Gnd & AGNDALLP & Analogue Pad Gnd \\
\hline 83 & IVFASTBUF & Analog I/O pad, $0 \Omega$ serial & APRIOP & DAC Test Purpose Only \\
\hline 84 & $\begin{array}{ll}\text { VDDA } \\
\end{array}$ & Analogue Pad Supply & AVDDALLP & VDDA \\
\hline 85 & IFASTINTBUF & Analog I/O pad, $0 \Omega$ serial & APRIOP & DAC Test Purpose Only \\
\hline 86 & GND & Analogue Pad Gnd & AGNDALLP & Analogue Pad Gnd \\
\hline 87 & IREGSLOWBUFDIF & Analog I/O pad, $0 \Omega$ serial & APRIOP & DAC Test Purpose Only \\
\hline 88 & VDDA & Analogue Pad Supply & AVDDALLP & VDDA \\
\hline 89 & IVREGSLOWBUFDIF & Analog I/O pad, $0 \Omega$ serial & APRIOP & DAC Test Purpose Only \\
\hline 90 & GND & Analogue Pad Gnd & AGNDALLP & Analogue Pad Gnd \\
\hline 91 & GND & Analogue Pad Gnd & AGNDALLP & Analogue Pad Gnd \\
\hline 92 & ISLOWBUFDIF & Analog I/O pad, $0 \Omega$ serial & APRIOP & DAC Test Purpose Only \\
\hline 93 & VDDA & Analogue Pad Supply & AVDDALLP & VDDA \\
\hline 94 & VDDA & Analogue Pad Supply & AVDDALLP & VDDA \\
\hline 95 & ISLOWBUFSE & Analog I/O pad, $0 \Omega$ serial & APRIOP & DAC Test Purpose Only \\
\hline$\overline{96}$ & GND & Analogue Pad Gnd & AGNDALLP & Analogue Pad Gnd \\
\hline 97 & GND & Analogue Pad Gnd & AGNDALLP & Analogue Pad Gnd \\
\hline 98 & IAMP & Analog I/O pad, $0 \Omega$ serial & APRIOP & DAC Test Purpose Only \\
\hline 99 & VDDA & Analogue Pad Supply & AVDDALLP & VDDA \\
\hline 100 & VDDA & Analogue Pad Supply & AVDDALLP & VDDA \\
\hline 101 & IREGAMP & Analog I/O pad, $0 \Omega$ serial & APRIOP & DAC Test Purpose Only \\
\hline 102 & GND & Analogue Pad Gnd & AGNDALLP & Analogue Pad Gnd \\
\hline 103 & GND & Analogue Pad Gnd & AGNDALLP & Analogue Pad Gnd \\
\hline 104 & IVREGAMP & Analog I/O pad, $0 \Omega$ serial & APRIOP & DAC Test Purpose Only \\
\hline 105 & VDDA & Analogue Pad Supply & AVDDALLP & VDDA \\
\hline 106 & VDDA & Analogue Pad Supply & AVDDALLP & VDDA \\
\hline 107 & IVTEST0 & Analog I/O pad, $0 \Omega$ serial & APRIOP & DAC Test Purpose Only \\
\hline 108 & \begin{tabular}{|l|l} 
GND \\
\end{tabular} & Analogue Pad Gnd & AGNDALLP & Analogue Pad Gnd \\
\hline 109 & GND & Analogue Pad Gnd & AGNDALLP & Analogue Pad Gnd \\
\hline 110 & IVTEST1 & Analog I/O pad, $0 \Omega$ serial & APRIOP & DAC Test Purpose Only \\
\hline 111 & VDDA & Analogue Pad Supply & AVDDALLP & VDDA \\
\hline 112 & VDDA & Analogue Pad Supply & AVDDALLP & VDDA \\
\hline 113 & IPIX & Analog I/O pad, $0 \Omega$ serial & APRIOP & DAC Test Purpose Only \\
\hline 114 & GND & Analogue Pad Gnd & AGNDALLP & Analogue Pad Gnd \\
\hline 115 & GND & Analogue Pad Gnd & AGNDALLP & Analogue Pad Gnd \\
\hline 116 & IKIMO & Analog I/O pad, $0 \Omega$ serial & APRIOP & Gen Purpose DAC Output \\
\hline 117 & VDDA & Analogue Core Supply & AVDDALLP & VDDA \\
\hline 118 & VDDA & Analogue Core Supply & AVDDALLP & VDDA \\
\hline 119 & VREGSLOWBUFDIF & Analog I/O pad, $0 \Omega$ serial & APRIOP & DAC Test Purpose Only \\
\hline 120 & GND & Analogue Pad Gnd & AGNDALLP & Analogue Pad Gnd \\
\hline 121 & GND & Analogue Pad Gnd & AGNDALLP & Analogue Pad Gnd \\
\hline 122 & VREGAMP & Analog I/O pad, $0 \Omega$ serial & APRIOP & DAC Test Purpose Only \\
\hline 123 & VDDA & Analogue Core Supply & AVDDALLP & VDDA \\
\hline 124 & \begin{tabular}{|l|} 
VDD_DIODE \\
\end{tabular} & Direct Pad, no protections & Full Custom & Pixel Diode Bias, 3.3 V \\
\hline 125 & \begin{tabular}{|l} 
GND \\
\end{tabular} & Analogue Pad Gnd & AGNDALLP & $\begin{array}{l}\text { Analogue Pad Gnd } \\
\end{array}$ \\
\hline
\end{tabular}




\section{Appendix III - HFT Prototype Read Out}

The design for the STAR prototype HFT read out is based on the daughter board designed by Fred Bieser and Robin Gareus and the synchronous cluster identifier and data compression scheme proposed by Leo Greiner. The advantages of this design are:

- The daughter board hardware will provide the full data rate and functionality required for the prototype $4 \mathrm{~ms}$ HFT.

- Much of the required VHDL firmware is running, tested and understood.

- The cluster identifier runs at the same speed as digitization providing immediate data compression. Only cluster center addresses are passed on for data storage.

- Data digitization and compression takes the same $4 \mathrm{~ms}$ for all events independent of data.

- The cluster identifier and data compression fits well in the FPGA environment. It requires few resources and can be implemented with simple straightforward VHDL coding.

- The design is triggered and fits the standard DAQ design.

- All the hits for an event are stored directly for that event. There are no complications with frame boundaries or hits for an event located in different frames. This is important because file handling software used in STAR data analysis does not have to be altered to accommodate the HFT.

- The latency is $4 \mathrm{~ms}$, but the dead time is $1 \mathrm{~ms}$ matching the new TPC system.

- The HFT data size for an event is $90 \mathrm{kB}$, significantly less than the $2 \mathrm{MB}$ for TPC central collision event.

This document first provides a brief description of the system. Then, this section is followed with a diagram and a list, which describes the elements of the design. Finally, a section on a proposed data storage structure with expected data loads is discussed.

\section{System Design}

An HFT ladder has 10 MIMOSTAR-4 chips with $640 \times 640$ pixels on each chip. Each chip is divided in half with two parallel analogue, differential current output buffers. The chips are continuously clocked at $50 \mathrm{MHz}$, rastering repeatedly through all the pixels connecting them to the output buffer. Current to voltage buffers at the end of the ladder drive the analogue signals over twisted pairs (about a meter length) that are connected eventually to the daughter boards. The daughter boards contain an 8 channel ADC, two SRAM chips and an FPGA. The daughter boards will handle digitization, zero suppression and data compression. For each event, they will generate a list of hits giving the address of the pixels at the cluster centers. They will also be able to operating in slow diagnostic mode providing amplitudes for all the pixels. The daughter board with its 8 ADC channels can handle 4 MIMOSTAR-4 chips. Using 3 daughter boards per ladder leaves 4 channels unused. 
The daughter boards function in the following manner. A common $50 \mathrm{MHz}$ clock that drives the MIMOSTAR-4 chip drives the ADCs at 50 MSPS digitizing one pixel after the other. Each ADC channel writes a 10 bit digital amplitude to a circular memory buffer repeatedly cycling through the $640 \times 320$ pixels on half of a MIMOSTAR- 4 chip. The ADCs have 12-bit capability, so we may use more than 10 bits.

The baseline voltage of the pixels varies significantly from pixel to pixel - much more than the amplitude of the minimum ionizing signal. To extract a signal the pixel must be digitized twice, once before and once after the particle hits the pixel. The signal is obtained from the difference in the two values - a method called Correlated Double Sampling (CDS). CDS is accomplished in this design by taking the difference between the old value saved on the circular buffer and the current ADC value. This is done before overwriting the circular buffer with the new ADC value. The CDS value or hit amplitude is then checked against a high and low threshold and the result is clocked into a shift register, again with the same common clock.

The shift register is long enough to contain 2 rows of the MAPS plus an additional 3 pixels into the third row. The purpose of the shift register is to provide a simple fast way to slide a 3 by 3 window over every pixel of the MAPS to test for clusters. Ports on the nine desired shift register cells are permanently connected to the cluster sensor (see Figure 1). Demanding a high threshold in the center pixel plus at least one low threshold in at least one neighbor pixel should be sufficient to find the center reliably. This has been checked with existing data and found to be a suitable approach. In addition to finding the center pixel, the purpose is to be able to detect minimum ionizing hits with $98 \%$ efficiency while limiting false hits from noise to a few 10 s of hits per cycle through the detector. Since the noise is essentially random, requiring two pixels above threshold instead of just one significantly reduces accidentals. This will also be a good filter against single hot pixels. However, if a hot pixel map is required, it can be stored in another bit on the circular buffer and this can be interrogated during CDS.

If the cluster test is positive, then the address of the pixel gets recorded into a FIFO whenever the trigger enables the FIFO. By timing from the trigger, a fixed width acceptance window with a fixed delay is generated. The window width is just the time required to cycle through all the pixels once. This allows all the hits associated with the trigger event to be recorded into the FIFO. With this continuous raster scan view a time window provides the simple selection of event hits. There is no complication of event hits spanning separate frames.

Once all of the hits for the event are recorded into the FIFO then the FIFOs for all the channels are read, assembled, and cleared by the mother board and sent over the RORC/DLL to the STAR DAQ system. The event acceptance window for an event is the same for all channels and can be generated at a single location. As soon as the acceptance window is closed the FIFOs are ready for reading. 


\section{Block Diagram}

The system shown in the Figure 1 will have a dead time of a little more than $4 \mathrm{~ms}$, but this can be significantly reduced with a reactively simple addition. Instead of one FIFO per channel, we will use 5. Each FIFO for the channel will have a separate trigged acceptance window generator. This approach will result in some data duplication, but it has the advantage that each event will be self contained, greatly simplifying file handling during data analysis. Data duplication is not an issue because the data volume is relatively small. 


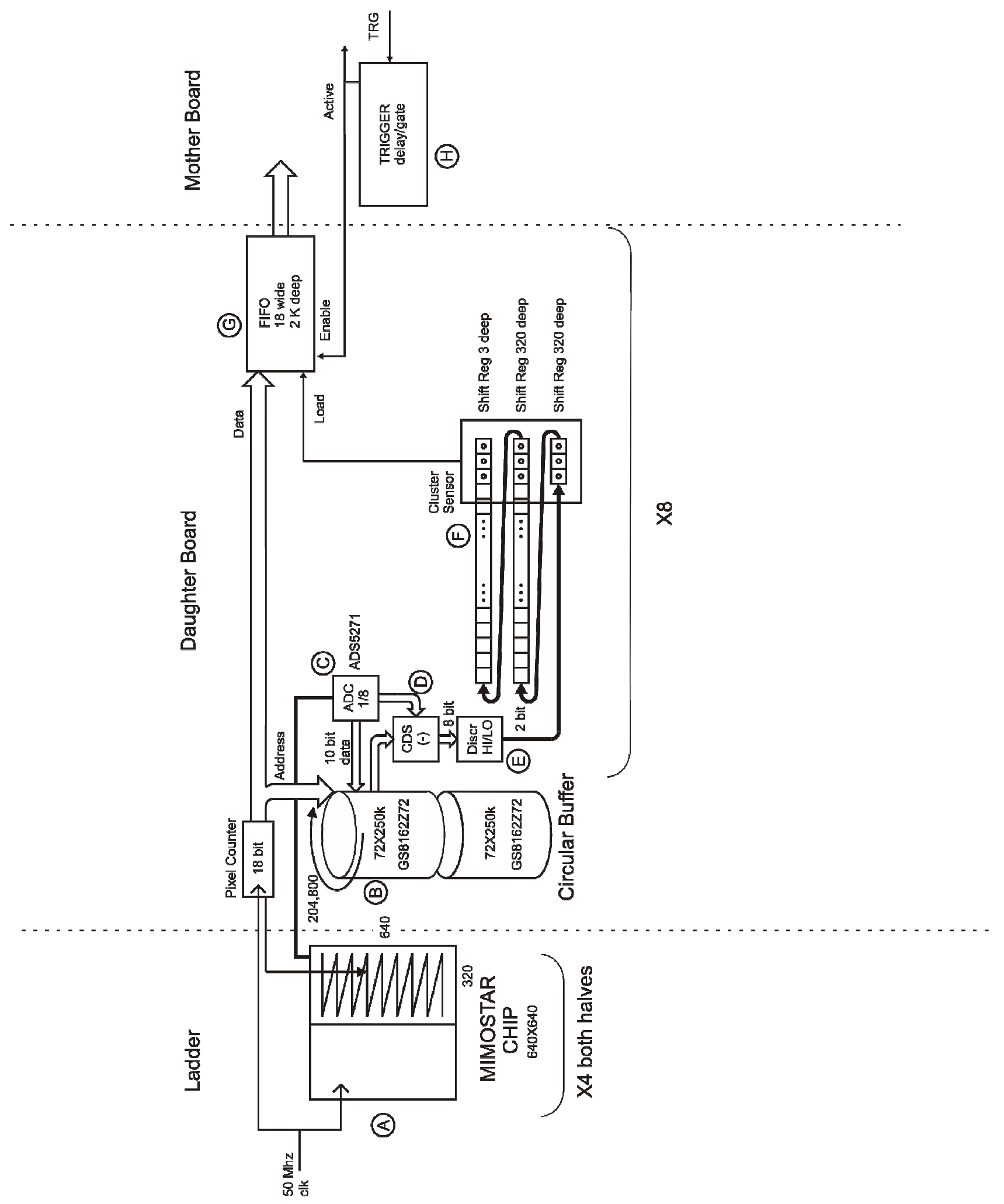

Fig. 1. Schematic for the readout system 
A. MIMOSTAR MAPS, $640 \times 640$ pixels. Continuous parallel raster scan readout of the two halves at $50 \mathrm{MHz}$ pixel rate.

B. Two SRAM memory chips, 72 bits wide and $256 \mathrm{~kb}$ deep, rated at 150-333 MHz, operated as a circular buffer addressed by the pixel counter looping over $320 \times$ $640=204,800$ locations. Up to 18 bits available in the memory for each pixel, but only 10 are required for the ADC value plus a possible $11^{\text {th }}$ bit used as a hot pixel marker. Two memory chips to handle 4 MIMOSTAR chips. VHDL code for memory control currently operating. GSI Technology http://gsitechnology.com/18MbBurst.htm

C. ADC chip with 8 parallel channels of 12 bit ADCs. Each ADC channel continuously digitizes the signals from one half of a MIMOSTAR chip at 50 MSPS. The output of each channel is serial LVDS. The ADCs are currently operating on the daughter board, but not at the required data rate. The firmware used was adapted by Robin Gareus from code for another FPGA and is based on work appearing in: http://direct.xilinx.com/bvdocs/appnotes/xapp774.pdf. Higher speed grades of the FPGA may provide speed sufficient for deserialization at our desired data rate. The ADC on the daughter board is an ADS5270 capable of 40 MSPS. The ADS5271 operates at 50 MSPS - see Texas Instruments' web site http://focus.ti.com/docs/prod/folders/print/ads5270.html

D. Correlated Double Sampling (CDS) is accomplished at full readout speed by reading the old pixel $\mathrm{ADC}$ value from the circular buffer and subtracting it from the current new ADC value. The new ADC value is written back to the circular buffer overwriting the old value.

E. A digital discriminator viewing the CDS value returns either 0, over low threshold or over high threshold and delivers the result to a shift register.

F. A cluster detector following Leo Greiner's design uses shift registers to check for clusters centered on each pixel at the full chip readout speed. The signal to noise is supposed to be sufficient to identify clusters just by a single pixel over high threshold. So, a simple center pixel over high threshold with an adjacent vertical or horizontal neighbor over low threshold may provide more than enough sensitivity to cleanly identify cluster centers. The goal is to be $98 \%$ efficient for minimum ionizing with less than a few tens of false hits per half chip. All 9 pixels (the center pixel and all its adjacent pixels) are available for a more complicated sensor algorithm, but the proposed simple scheme should be adequate. There may be sufficient filtering to forgo using a hot pixel map. Note, however, the proposed daughter board architecture lends its self to using a hot pixel map. There is just the added complication of uploading the map to the circular buffer. In the figure the shift registers are shown as 3 separate items, but in practice they may well be implemented as a single register ported as required. The cluster sensor/ shift registers, digital discriminator (E), CDS (D), and ADC channel $(\mathrm{C})$ are repeated 8 times. This should be well within the resources of the Xilinx Vertex-II FPGA, XC2V1000 current used on the daughter board http://www.xilinx.com/products/silicon solutions/fpgas/virtex/virtex ii platform fpgas/product table.htm. The selected FPGA has a total of $720 \mathrm{~kb}$ Block RAM and $160 \mathrm{~kb}$ distributed RAM. The shift registers require only $10 \mathrm{~kb}$. 
G. When the cluster sensor detects a cluster it loads (if trigger enabled) the center pixel address (the pixel counter + a fixed offset) into a FIFO for export. This is the only data that gets exported. The FIFO shown is 18 bits wide as required to contain the pixel address for half the chip. Note the selected data loaded into the FIFO is the same as the address used to access the circular buffer. The expected number of hits on the inner ladders at $10^{27} \mathrm{~Hz} / \mathrm{cm}^{2}$ luminosity is $\sim 200$ for half a chip, so the $2 \mathrm{k}$ deep FIFO is generous. This FIFO is repeated 8 times for a total Block RAM requirement of $288 \mathrm{~kb}$. This is well within the $720 \mathrm{~kb}$ available on the current daughter board. The system shown will operate triggered with a $4 \mathrm{~ms}$ dead time. As discussed, a faster $1 \mathrm{~ms}$ dead time system will require 5 times as many FIFOs or $1440 \mathrm{~kb}$ which is over the XC2V1000 limit. A bigger FPGA, the XC2V3000, however would suffice or the FIFO depth could be reduced by half which is still 5 times the expected load.

H. The trigger, signal, which arrives $1 \mu$ s after the event collision of interest, is delayed and stretched setting the recording window for storing cluster center addresses into the FIFO. The delay is set to 322 clock cycles less the 1 micro second trigger delay. This total delay is the time required for the first hit pixel of the event to ripple through the shift register to the center of the cluster sensor. The stretch time (acceptance window width) is 204,800 clock cycles, the time required for the last potentially struck pixel of the event to reach the center of the cluster sensor. These numbers can be changed to exclude pixels in the edge rows being counted as cluster centers. In this scheme all the clusters for the triggered collision are recorded. The dead time is roughly $4 \mathrm{~ms}$ the time required to read through all the pixels once. Some additional dead time may result in transferring the FIFO data down to the STAR DAQ system. Additional collisions occur during the $4 \mathrm{~ms}$ read time and these background clusters are included. A simple variation on this scheme can be used to reduce the dead time so that the HFT can be included with every TPC trigger. This is done by adding four more FIFOs and trigger stretchers for each ADC/cluster finder channel. When a FIFO is accepting cluster addresses from one event and a new trigger occurs, the next available FIFO/trigger stretcher can process data. The acceptance windows of the two FIFOs will partially overlap, but each event will carry all the clusters associated with the event. The addition greatly simplifying STAR data analysis.

In the design outlined some event building and trigger handling gets done on the mother board. This differs from the current ladder prototype design where there are no FPGAs on the mother board. All data communication is handled by the daughter board FPGA. The advantage in this approach of using the daughter board FPGA for all the functions is the simplification of VHDL code development. Having all the code in one place for debugging and maintenance is certainly desirable. More thought will be given to following this example. Perhaps the trigger functions and event building can also be accomplished in the daughter board FPGA. 
In any case additional, work is required on for the data connection to the outside. Currently the connection is via Robin Gareus' PCI connection protocol and his slow connection code scsn. We want to use instead the RORC/DLL connection that has become the STAR standard. We are considering implementing a USB connection to LabVIEW, which provides a more portable operation for testing and debugging.

\section{Data rates, zero suppression, data structure}

The data reduction achieved in the daughter board with this design is significant. For a half a chip, data rate is $65 \mathrm{MB} / \mathrm{s}$ before CDS and $50 \mathrm{MB} / \mathrm{s}$ after CDS. After cluster identification, the rate depends on hit density and luminosity. At a luminosity of $10^{27}$ $\mathrm{Hz} / \mathrm{cm}^{2}$, the data rate for the $1 / 2$ chip in the inner radius is $0.5 \mathrm{MB} / \mathrm{s}$ which translates to an event rate of $1 \mathrm{kHz}$. This is a reduction of over 100 for the inner, high exposure chips. The reduction is more for the outer layer.

$18 \mathrm{~b}$ wide

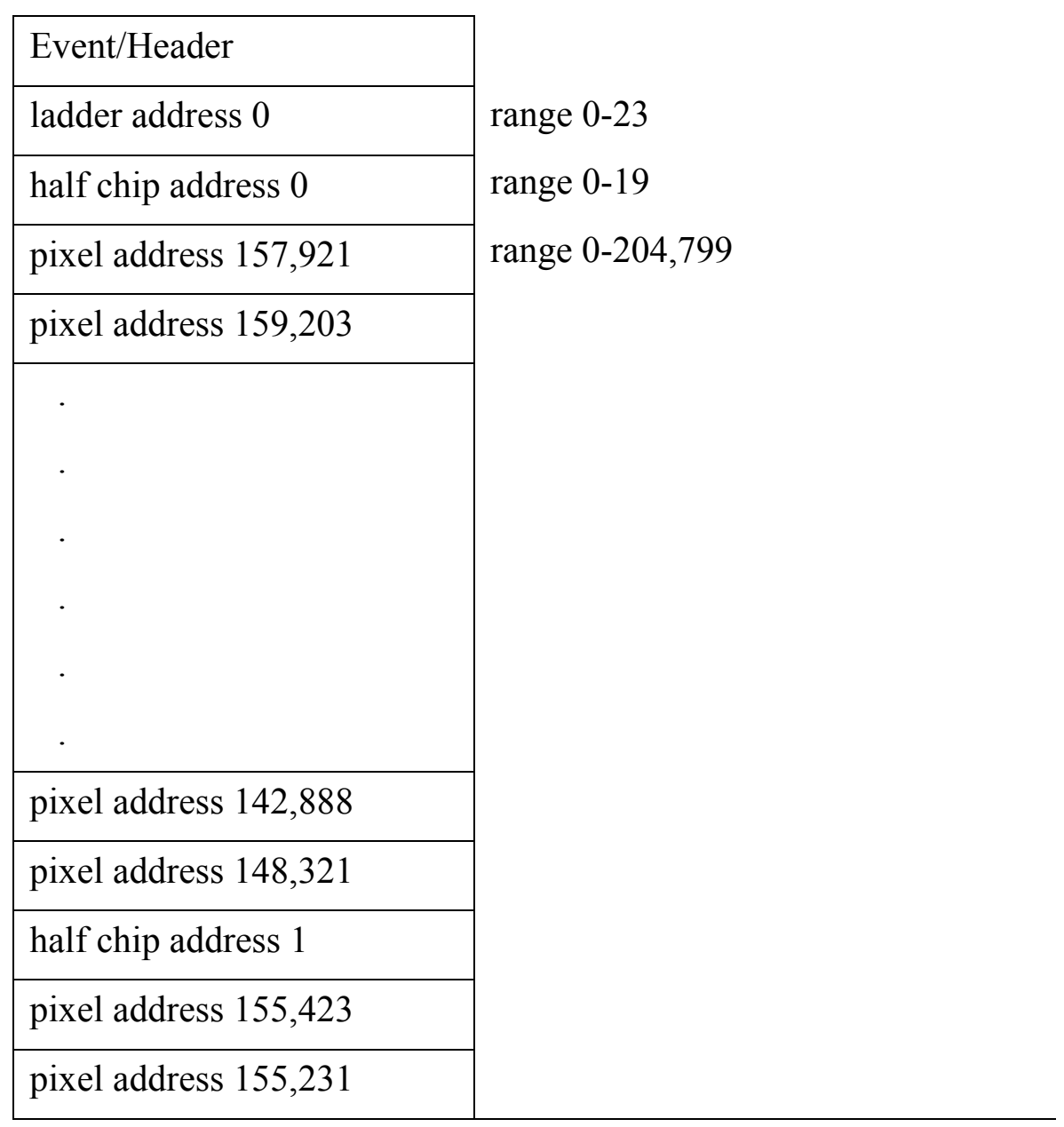




\begin{tabular}{|l|}
\hline$\cdot$ \\
$\cdot$ \\
$\cdot$ \\
\hline \\
\hline ladder address 1 \\
\hline half chip address 0 \\
\hline$\cdot$ \\
\hline$\cdot$ \\
\hline
\end{tabular}

Fig. 2. Data structure for HFT

The total data volume depends on the data structure used. A proposed data structure with example population is shown in Fig. 2. Half chip addresses and ladder addresses are distinguished from pixel addresses by adding constants to make them larger than the maximum pixel address of 204,799. If it is desirable to format the data into 3 bytes instead of 18 bits, then the additional bits may be used to distinguish the 3 address types directly.

As calculated in Table 1, the HFT event size of $90 \mathrm{kB}$ is significantly smaller than the $\mathrm{TPC}$, which has an event size of $2 \mathrm{MB}$ for central $\mathrm{Au}+\mathrm{Au}$. This proposed data format of ordered hits can be compared with one that saves the hit addresses. The event storage size for the address case is $12 \mathrm{MB}$, over 100 times larger than the proposed scheme.

\begin{tabular}{|l|l|}
\hline Item & Value \\
\hline bits/address & 18 \\
\hline inner ladders & 6 \\
\hline outer ladders & 18 \\
\hline half chips per ladder & 20 \\
\hline ave hits/half chip, inner, $\mathrm{L}=10^{27} \mathrm{~Hz} / \mathrm{cm}^{2}$ & 200 \\
\hline ave hits/half chip, outer, $\mathrm{L}=10^{27} \mathrm{~Hz} / \mathrm{cm}^{2}$ & 40 \\
\hline Event Size & $90 \mathrm{kB}$ \\
\hline Data Rate at $1 \mathrm{KHz}$ event rate & $90 \mathrm{MB} / \mathrm{sec}$ \\
\hline
\end{tabular}

Table 1. Readout numbers for the HFT 


\section{Appendix IV - Production Schedule}

\begin{tabular}{|l|l|l|}
\hline Sensor & Item & Date \\
\hline MIMOSTAR-1 & Begin design & Summer 2003 \\
\hline \multirow{4}{*}{ MIMOSTAR-2 } & Production & 2004 \\
\hline \multirow{4}{*}{ MIMOSTAR-3 } & Beam Test \#1 at DESY & November 2005 \\
\cline { 2 - 3 } & Beam Test \#2 at DESY & June/July 2006 \\
\cline { 2 - 3 } & Install in STAR & Fall 2006 \\
\cline { 2 - 3 } & Sent to production & June 2006 \\
\cline { 2 - 3 } & Probe tests & September 2006 \\
\cline { 2 - 3 } & Dicing and thinning & October 2006 \\
\cline { 2 - 3 } & Mounting on a ladder & December 2006 \\
\hline MIMOSTAR-4 & Back from foundry & August 2007 \\
\cline { 2 - 3 } & Dicing and thinning & September 2007 \\
\cline { 2 - 3 } & Testing of chips & October 2007 to Early 2008 \\
\hline \multirow{2}{*}{ Ultimate } & Early exploration & 2006 \\
\cline { 2 - 3 } & Production version & 2009 \\
\hline
\end{tabular}

From "Summary of the STAR meeting at IReS-Strasbourg on January 18-20, 2008", M. Winter 


\section{Appendix V - Cost and Schedule Presentation}

Cost and Schedule estimates presented to the Technical Advisory Committee for RHIC detector upgrades on March 15, 2006. 

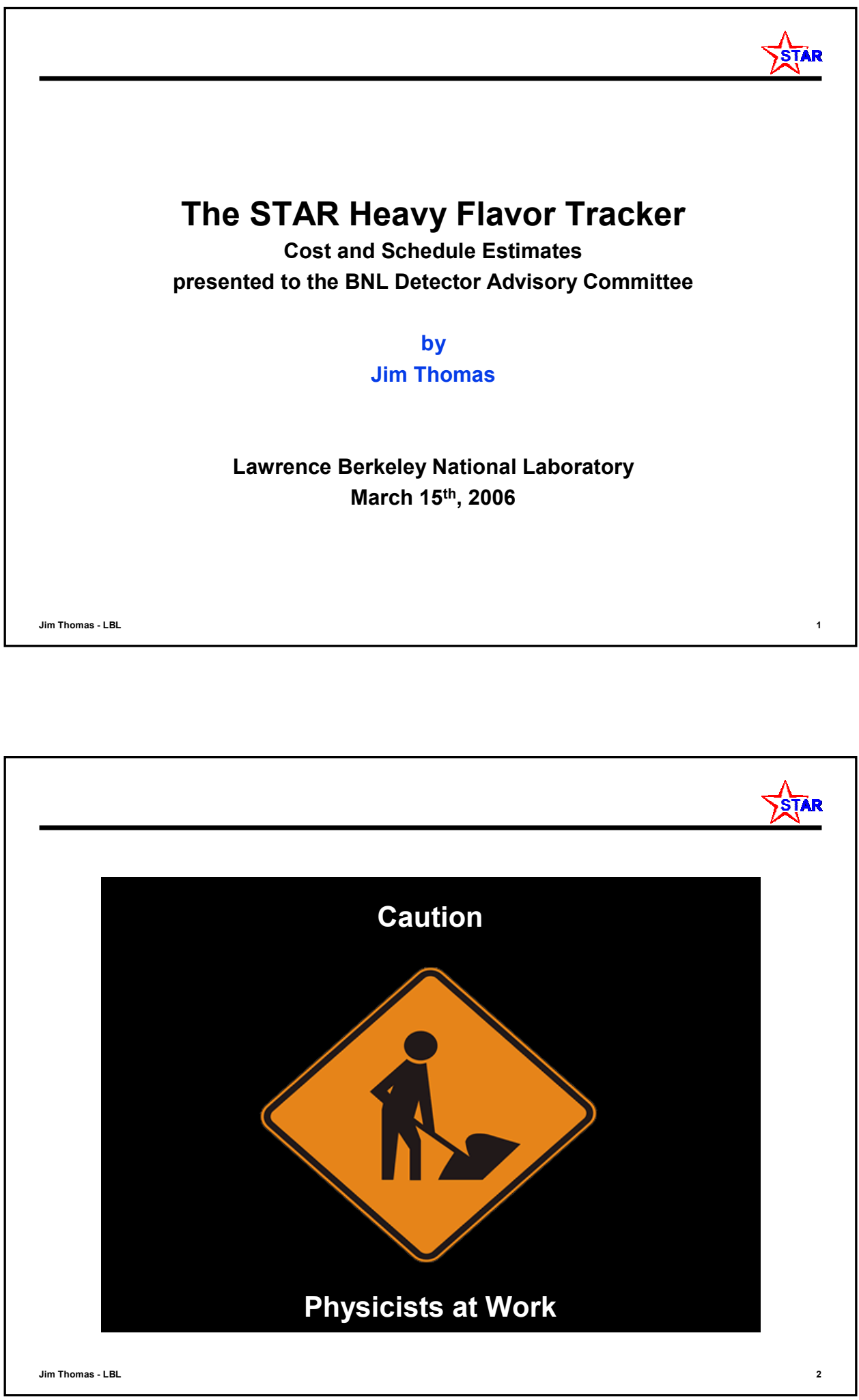
Driven by the availability of CMOS Active Pixel Sensors
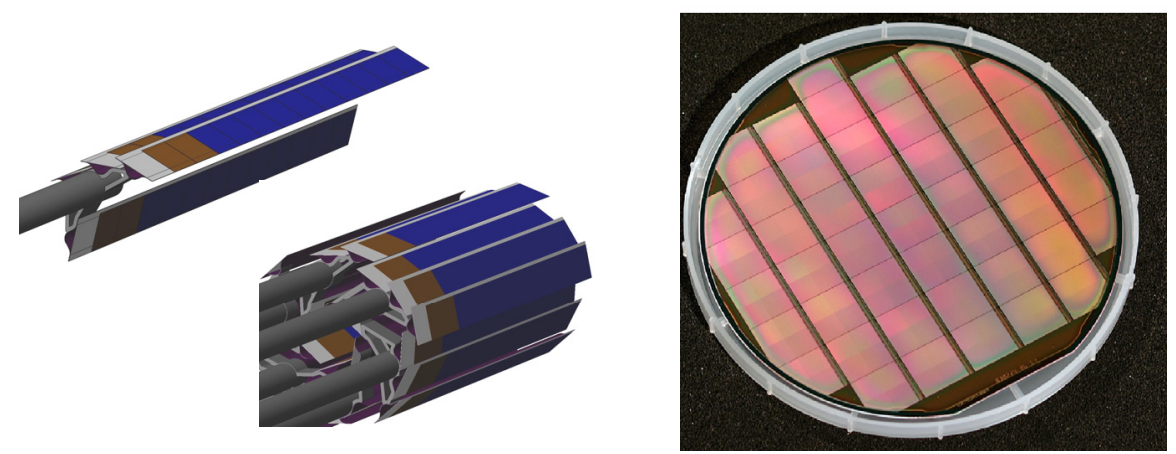

\begin{tabular}{|c|c|c|c|c|c|c|c|}
\hline 1999 & 2001 & 2003 & 2004 & 2005 & 2006 & 2007 & 2009 \\
\hline Mimosa-1 & Mimosa-4 & Mimosa-8 & MimoSTAR-1 & MimoSTAR-2 & MimoSTAR-3 & MimoSTAR-4 & UltraSTAR \\
\hline
\end{tabular}

\section{The Availability of Funds}

- The BNL Mid-Term Plan includes funding for the HFT

Proposed HFT Profile

$\begin{array}{ccccc}06 & 07 & 08 & 09 & 10 \\ 300 \mathrm{~K} & 1 \mathrm{M} & 800 \mathrm{~K}+300 \mathrm{~K} & 2.5 \mathrm{M} & 2.5 \mathrm{M} \\ \text { R\&D } & \text { R\&D } & \text { R\&D+Const } & \text { Const } & \text { Const }\end{array}$

- The proposed schedule of funds makes Mimosa-8 technology available in time to complete the project

- The project has the $200 \mu \mathrm{sec}$ readout chip as the final goal

- The R\&D profile allows us to complete the development of the MimoSTAR chips and to readout data with a $4 \mathrm{msec}$ frame rate

- Do extensive R\&D and testing with MimoSTAR-4 chips

- Mount them in STAR

- Use the real beam pipe, real beam rates, real background

- Use the real mechanical insertion device

- The Construction Profile allows us to complete the development of the Mimosa-8 style chips and readout with $200 \mu \mathrm{sec}$ frame rate

- The final detector will be based on UItraSTAR chips 
- Challenging HFT technologies

- The Silicon Chips

- Further refinement of on-chip electronics

- Readout Electronics

- speed, heat dissipation, compatibility with STAR DAQ

- The Mechanical Arms to insert the detector

- Alignment and stability

- Calibration, Tracking \& Software

- New levels of precision

- The beam pipe

- Smaller than ever before ... Operation and robustness

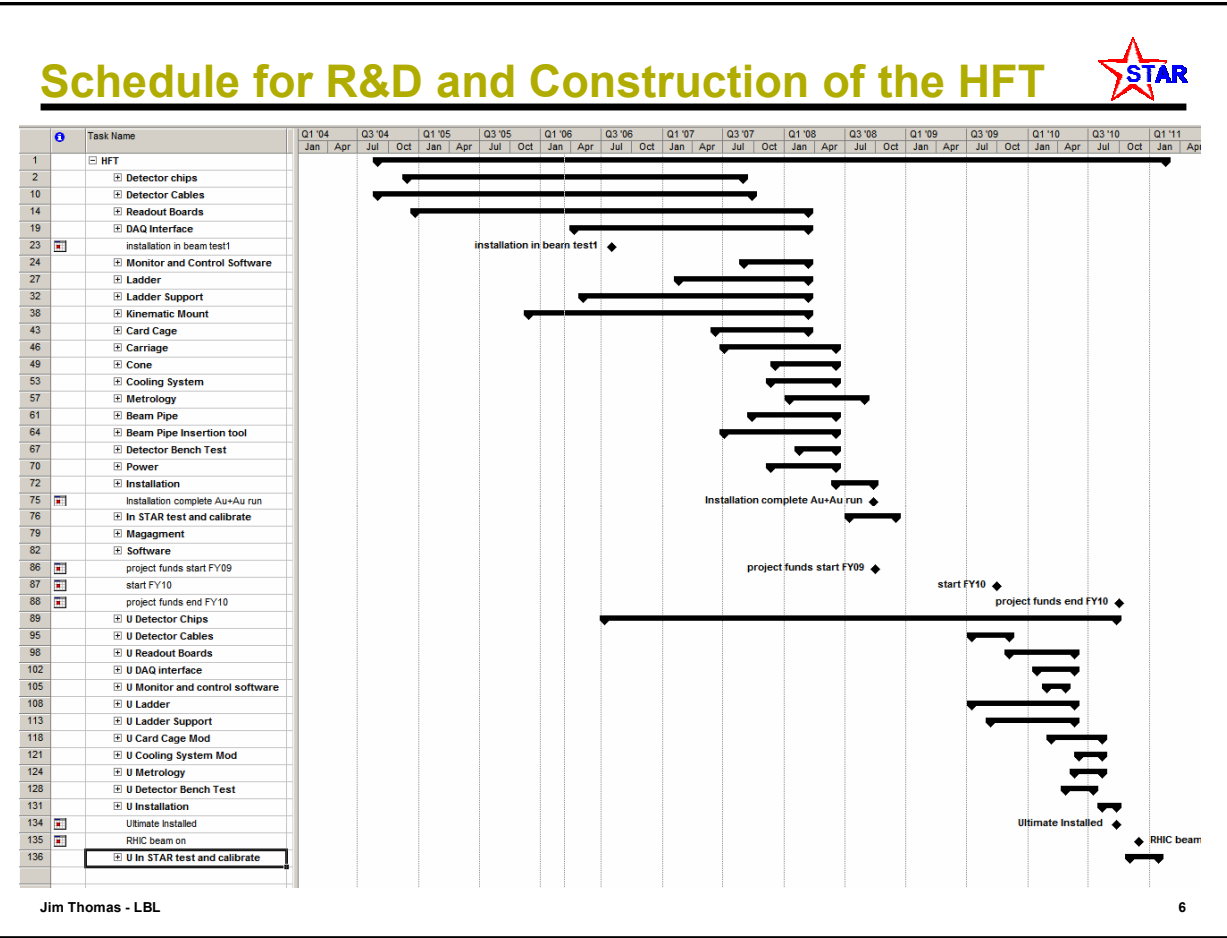




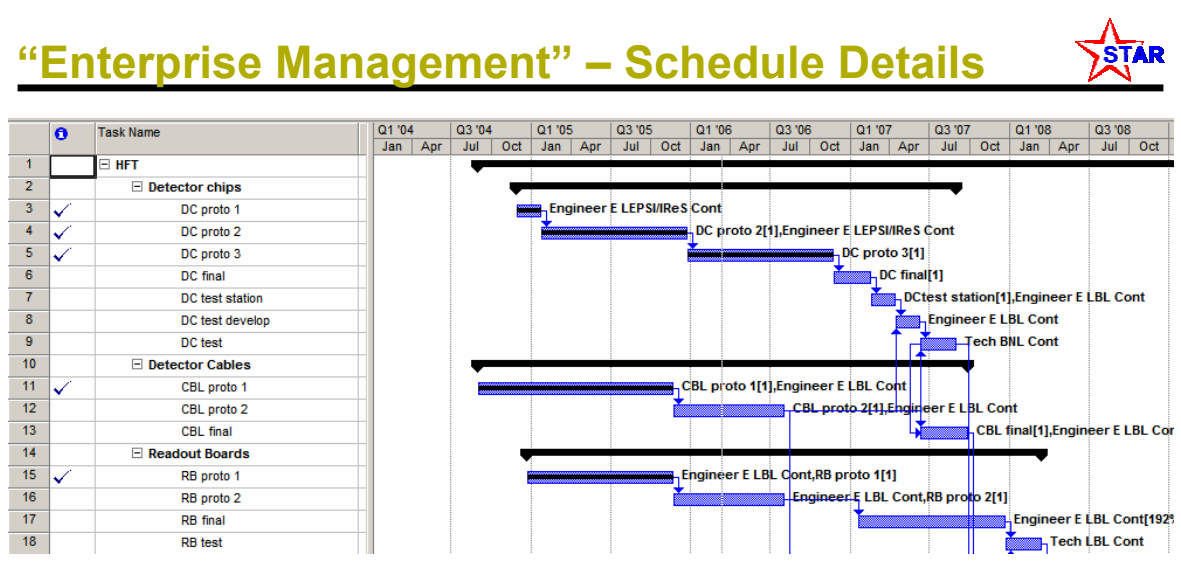

- The Gantt Chart tracks the full enterprise of activities

- not a 'project' Gantt chart because it tracks more than the 'project'

- some pre-conceptual design activities are included and complete

- we do not track, or cost out, the IRES contributions

- For the most part, it's a conventional project with R\&D and construction activities tracked across time

- Engineering, technical, and contributed labor are included

\section{IRES/IPHC-Strasbourg Contributions}

- Six years of Experience developing CMOS Active Pixel Sensors

- Long term goal of developing sensors for the ILC, CBM and STAR

- Outstanding staff and students

- 10 Engineers

- 3 Physicists

- 7 Ph.D. students

- Commitment to the project

- MimoSTAR-1, -2, -3 have consumed 2 physicists and 3 engineers over the past 2 years

- Financial equivalent value of their contribution

- Approximately \$2 Million so far

Strasbourg Contributions: Not costed to the Project 
- MimoSTAR-1

- MimoSTAR-2

- MimostAR-3

- MimoSTAR-4

Approximately one

generation each year

- We pay partial cost for R\&D and test runs

- Share mask costs

- Share space on the wafers

- We pay full cost for production runs

- For a non-production run, LBL has be paying $30 \mathrm{~K}-50 \mathrm{~K}$ per run

- IRES has been absorbing or sharing the remainder of the costs

\section{Silicon Production Costs for Chips

\begin{tabular}{|l|l|}
\hline Chips per ladder & 10 \\
\hline Ladders per Detector & 24 \\
\hline Number of Detector Copies & 4 \\
\hline Number of working chips & 960 \\
\hline Yield & $60 \%$ \\
\hline Total chips & 1600 \\
\hline Total wafers & 27 \\
\hline Wafer Cost Each & $7.2 \mathrm{k \$}$ \\
\hline Wafer Costs & $195 \mathrm{k} \$$ \\
\hline Mask Cost & $220 \mathrm{k} \$$ \\
\hline Total & $415 \mathrm{k} \$$ \\
\hline
\end{tabular}

8 inch wafers

60 chips/wafer

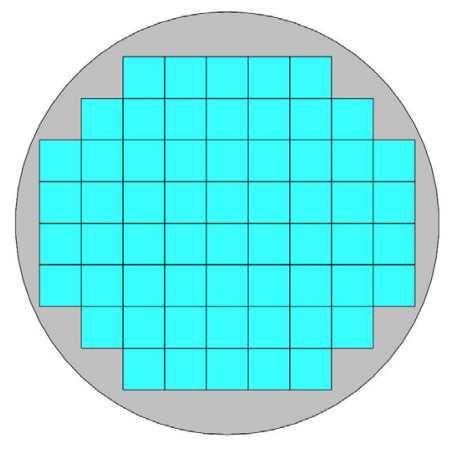




\section{Contingency Levels used on the Gantt Chart}

- Contingency on Si

- $50 \%$ on fabrication costs

- Contingency on Engineering

- $50 \%$ for the first time a component is designed

- $25 \%$ if there has been a prior (significant) R\&D test

- pertains to Ultra Chip

- Contingency on Hardware procurements

$-75 \%$

- Contingency on Fabricated elements (in house)

$-75 \%$

- MimoSTAR IV chips (4 msec readout)

- two copies for R\&D work

- UltraSTAR Chips (200 $\mu \mathrm{sec}$ readout)

- four copies for the MIE Detector

- Readout electronics

- $100 \%$ spares for the MIE Detector 


\begin{tabular}{|l|l|l|l|}
\hline & Base Rate & $\begin{array}{l}\text { With } \\
\text { OverHead }\end{array}$ & $\begin{array}{l}\text { With 75\% } \\
\text { Contingency }\end{array}$ \\
\hline Engineering & $65.60 / \mathrm{hr}$ & $132.00 / \mathrm{hr}$ & $231 / \mathrm{hr}$ \\
\hline Technical & $43.75 / \mathrm{hr}$ & $88.00 / \mathrm{hr}$ & $154 / \mathrm{hr}$ \\
\hline
\end{tabular}

\section{Cost of Materials}

\begin{tabular}{|c|c|c|c|c|c|c|c|}
\hline & Resource Name & Cost & Baseline Cost & Variance & Actual Cost & Standard & Cost Per Use \\
\hline & $\square$ Type: Material & $\$ 2,745,841.78$ & $\$ 0.00$ & $\$ 2,745,841.78$ & $\$ 170,830.00$ & & $\$ 2,818,441.78$ \\
\hline 41 & BP procur & $\$ 288,750.00$ & $\$ 0.00$ & $\$ 288,750.00$ & $\$ 0.00$ & $\$ 0.00$ & $\$ 288,750.00$ \\
\hline 36 & CA fab & $\$ 3,850.00$ & $\$ 0.00$ & $\$ 3,850.00$ & $\$ 0.00$ & $\$ 0.00$ & $\$ 3,850.00$ \\
\hline 21 & CBL final & $\$ 38,500.00$ & $\$ 0.00$ & $\$ 38,500.00$ & $\$ 0.00$ & $\$ 0.00$ & $\$ 38,500.00$ \\
\hline 19 & CBL proto 1 & $\$ 19,250.00$ & $\$ 0.00$ & $\$ 19,250.00$ & $\$ 19,250.00$ & $\$ 0.00$ & $\$ 19,250.00$ \\
\hline 20 & CBL proto 2 & $\$ 19,250.00$ & $\$ 0.00$ & $\$ 19,250.00$ & $\$ 0.00$ & $\$ 0.00$ & $\$ 19,250.00$ \\
\hline 22 & CBL rework & $\$ 0.00$ & $\$ 0.00$ & $\$ 0.00$ & $\$ 0.00$ & $\$ 0.00$ & $\$ 3,850.00$ \\
\hline 35 & $\mathrm{CC}$ fab & $\$ 3,850.00$ & $\$ 0.00$ & $\$ 3,850.00$ & $\$ 0.00$ & $\$ 0.00$ & $\$ 3,850.00$ \\
\hline 37 & CONE procur & $\$ 3,850.00$ & $\$ 0.00$ & $\$ 3,850.00$ & $\$ 0.00$ & $\$ 0.00$ & $\$ 3,850.00$ \\
\hline 38 & COOL procur & $\$ 7,700.00$ & $\$ 0.00$ & $\$ 7,700.00$ & $\$ 0.00$ & $\$ 0.00$ & $\$ 7,700.00$ \\
\hline 27 & DAQ Purch & $\$ 19,250.00$ & $\$ 0.00$ & $\$ 19,250.00$ & $\$ 0.00$ & $\$ 0.00$ & $\$ 19,250.00$ \\
\hline 17 & $\mathrm{DC}$ final & $\$ 451,687.50$ & $\$ 0.00$ & $\$ 451,687.50$ & $\$ 0.00$ & $\$ 0.00$ & $\$ 451,687.50$ \\
\hline 15 & DC proto 1 & $\$ 0.00$ & $\$ 0.00$ & $\$ 0.00$ & $\$ 0.00$ & $\$ 0.00$ & $\$ 26,400.00$ \\
\hline 16 & DC proto 2 & $\$ 29,700.00$ & $\$ 0.00$ & $\$ 29,700.00$ & $\$ 29,700.00$ & $\$ 0.00$ & $\$ 29,700.00$ \\
\hline 48 & DC proto 3 & $\$ 118,800.00$ & $\$ 0.00$ & $\$ 118,800.00$ & $\$ 118,800.00$ & $\$ 0.00$ & $\$ 118,800.00$ \\
\hline 18 & DCtest station & $\$ 19,250.00$ & $\$ 0.00$ & $\$ 19,250.00$ & $\$ 0.00$ & $\$ 0.00$ & $\$ 19,250.00$ \\
\hline 42 & Insertion parts & $\$ 3,850.00$ & $\$ 0.00$ & $\$ 3,850.00$ & $\$ 0.00$ & $\$ 0.00$ & $\$ 3,850.00$ \\
\hline 40 & Installation procur & $\$ 3,850.00$ & $\$ 0.00$ & $\$ 3,850.00$ & $\$ 0.00$ & $\$ 0.00$ & $\$ 3,850.00$ \\
\hline 34 & $\mathrm{KM}$ final & $\$ 5,775.00$ & $\$ 0.00$ & $\$ 5,775.00$ & $\$ 0.00$ & $\$ 0.00$ & $\$ 5,775.00$ \\
\hline 33 & KM proto & $\$ 1,925.00$ & $\$ 0.00$ & $\$ 1,925.00$ & $\$ 0.00$ & $\$ 0.00$ & $\$ 1,925.00$ \\
\hline 31 & $L$ final & $\$ 19,250.00$ & $\$ 0.00$ & $\$ 19,250.00$ & $\$ 0.00$ & $\$ 0.00$ & $\$ 19,250.00$ \\
\hline 30 & L proto & $\$ 9,625.00$ & $\$ 0.00$ & $\$ 9,625.00$ & $\$ 0.00$ & $\$ 0.00$ & $\$ 9,625.00$ \\
\hline 49 & LS final & $\$ 19,250.00$ & $\$ 0.00$ & $\$ 19,250.00$ & $\$ 0.00$ & $\$ 0.00$ & $\$ 19,250.00$ \\
\hline
\end{tabular}




\begin{tabular}{|c|c|c|c|c|c|c|c|}
\hline 32 & LS proto & $\$ 9,625.00$ & $\$ 0.00$ & $\$ 9,625.00$ & $\$ 0.00$ & $\$ 0.00$ & $\$ 9,625.00$ \\
\hline 39 & $M$ fab & $\$ 1,925.00$ & $\$ 0.00$ & $\$ 1,925.00$ & $\$ 0.00$ & $\$ 0.00$ & $\$ 1,925.00$ \\
\hline 28 & Monitor purch & $\$ 3,850.00$ & $\$ 0.00$ & $\$ 3,850.00$ & $\$ 0.00$ & $\$ 0.00$ & $\$ 3,850.00$ \\
\hline 26 & Power & $\$ 9,625.00$ & $\$ 0.00$ & $\$ 9,625.00$ & $\$ 0.00$ & $\$ 0.00$ & $\$ 9,625.00$ \\
\hline 25 & RB final & $\$ 385,825.44$ & $\$ 0.00$ & $\$ 385,825.44$ & $\$ 0.00$ & $\$ 0.00$ & $\$ 385,825.44$ \\
\hline 23 & RB proto 1 & $\$ 3,080.00$ & $\$ 0.00$ & $\$ 3,080.00$ & $\$ 3,080.00$ & $\$ 0.00$ & $\$ 3,080.00$ \\
\hline 24 & RB proto 2 & $\$ 3,080.00$ & 50.00 & $\$ 3,080.00$ & $\$ 0.00$ & $\$ 0.00$ & $\$ 3,080.00$ \\
\hline 29 & slow control Purch & $\$ 3,850.00$ & $\$ 0.00$ & $\$ 3,850.00$ & $\$ 0.00$ & $\$ 0.00$ & $\$ 3,850.00$ \\
\hline 43 & Software Purch & $\$ 30,030.00$ & $\$ 0.00$ & $\$ 30,030.00$ & $\$ 0.00$ & $\$ 0.00$ & $\$ 30,030.00$ \\
\hline 45 & Software training & $\$ 5,775.00$ & $\$ 0.00$ & $\$ 5,775.00$ & $\$ 0.00$ & $\$ 0.00$ & $\$ 5,775.00$ \\
\hline 50 & $\pi$ board & $\$ 105,875.00$ & $\$ 0.00$ & $\$ 105,875.00$ & $\$ 0.00$ & $\$ 0.00$ & $\$ 105,875.00$ \\
\hline 62 & U CBL final & $\$ 38,500.00$ & $\$ 0.00$ & $\$ 38,500.00$ & $\$ 0.00$ & $\$ 0.00$ & $\$ 38,500.00$ \\
\hline 64 & U CBL final & $\$ 0.00$ & $\$ 0.00$ & $\$ 0.00$ & $\$ 0.00$ & $\$ 0.00$ & $\$ 38,500.00$ \\
\hline 61 & U CBL proto 1 & $\$ 3,850.00$ & $\$ 0.00$ & $\$ 3,850.00$ & $\$ 0.00$ & $\$ 0.00$ & $\$ 3,850.00$ \\
\hline 63 & U CBL proto 1 & $\$ 0.00$ & $\$ 0.00$ & $\$ 0.00$ & $\$ 0.00$ & $\$ 0.00$ & $\$ 3,850.00$ \\
\hline 73 & U CC design & $\$ 1,925.00$ & $\$ 0.00$ & $\$ 1,925.00$ & $\$ 0.00$ & $\$ 0.00$ & $\$ 1,925.00$ \\
\hline 74 & U CC fab & $\$ 1,925.00$ & $\$ 0.00$ & $\$ 1,925.00$ & $\$ 0.00$ & $\$ 0.00$ & $\$ 1,925.00$ \\
\hline 75 & U COOL mod & $\$ 1,925.00$ & $\$ 0.00$ & $\$ 1,925.00$ & $\$ 0.00$ & $\$ 0.00$ & $\$ 1,925.00$ \\
\hline 68 & U DAQ final & $\$ 1,925.00$ & $\$ 0.00$ & $\$ 1,925.00$ & $\$ 0.00$ & $\$ 0.00$ & $\$ 1,925.00$ \\
\hline 67 & U DAQ proto & $\$ 1,925.00$ & $\$ 0.00$ & $\$ 1,925.00$ & $\$ 0.00$ & $\$ 0.00$ & $\$ 1,925.00$ \\
\hline 58 & U DC Final & $\$ 577,500.00$ & $\$ 0.00$ & $\$ 577,500.00$ & $\$ 0.00$ & $\$ 0.00$ & $\$ 577,500.00$ \\
\hline
\end{tabular}

- Engineering labor

$$
\sim 13.5 \text { FTEs }
$$

- Technical labor

$$
\sim 7.5 \text { FTEs }
$$

- Management \& Management support

$$
\sim 3 \text { FTEs }
$$

- Costed Labor

$$
\text { - Project } \quad 3 \mathrm{M}
$$

\section{- Contributed labor}

- BNL $\sim 1 \mathrm{M}$

- LBL $\quad \sim 2 \mathrm{M}$ 
- Significant activities to be studied in FY06 include:

- QA \& test of MimoSTAR II chips from Strasbourg

- Conceptual design of readout boards for the Strasbourg chips

- Prototype ladders to support the chips

- DAQ interface prototype

- Develop Al clad cable technology

- Conceptual Design of the kinematic mounts to hold the ladders

- R\&D for MimoSTAR III chip

- Expenses

$\sim$ 150K Procurements

$\sim 150 \mathrm{~K}$ Engineering

$\sim 600 \mathrm{~K}^{*}$ Contributed Engineering

* not realistic, needs refinement and buy-in from LBL management

- Significant activities to be studied in FY07 include:

- QA \& test of MimoSTAR III chips from Strasbourg

- Prototype readout boards for the Strasbourg chips

- Prototype ladders to support the chips

- Conceptual Design of the kinematic mounts to hold the ladders

- Live Beam tests in STAR using MimoSTAR II chips

- Initial discussion of the beam pipe

- Expenses

$\sim$ 670K Procurements

$\sim 250 \mathrm{~K}$ Engineering

$\sim 1500 K^{*}$ Contributed Engineering

* not realistic, needs refinement and buy-in from LBL management 


\section{R\&D Activities by Year}

- Significant activities to be studied in FY08 include:

- QA \& test of the MimoSTAR IV chips from Strasbourg

- Live Beam tests in STAR using MimoSTAR III chips and prototype kinematic mounts

- Integration studies for the support of the HFT and Cone modifications

- Development of alignment and calibration techniques

- Design and test of the thin walled beam pipe in STAR

- Develop and test interface to STAR DAQ

- R\&D for the Ultra Chip

- Expenses

$\sim 800 \mathrm{~K}$ Procurements

$\sim$ 900K Engineering

$\sim 1150 \mathrm{~K}^{*}$ Contributed Engineering

* not realistic, needs refinement and buy-in from LBL management

\section{Schematic view of Installation Dates $\quad$ SSTAR}

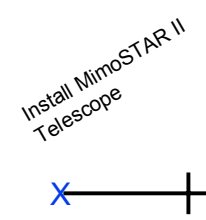

06
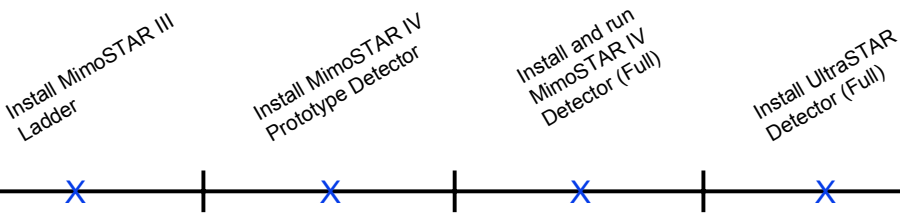

08

09

10

Installation is typically done in the summer of each year 
- Cable for Ultra Chips

3Q '09

- Ultra Chip testing complete

1Q'10

- Readout Boards complete

2Q'10

- DAQ Interface

2Q'10

- Ladder and Ladder Support

2Q'10

- Metrology

$3 Q$ '10

- Installation in STAR

$4 Q^{\prime} 10$

- RHIC Beam On

$1 \mathrm{Q} ` 11$

\section{Profile of Funds - "Houston, we have a problem" "\$NTAR}

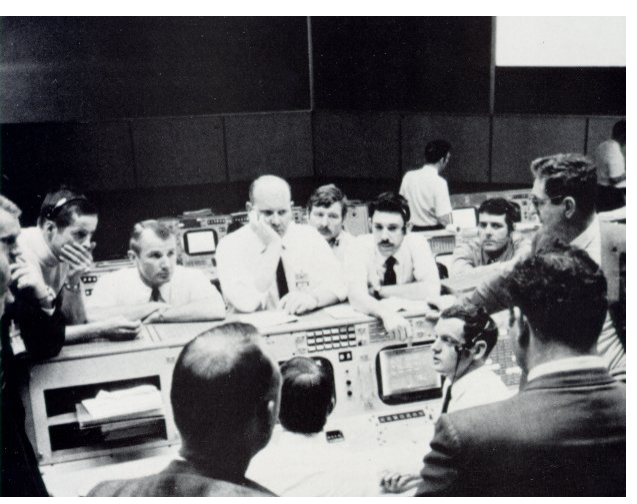

- In the spirit of being honest about our work ...

- The MS Project file is overly ambitious

- We are trying to do to more engineering studies than can be sustained in the R\&D era

- The LBL base program (to say the least) cannot support this much contributed effort

- However, the integral of funds is about right

- I believe the profile problem can be solved by delaying some of the mechanical design but I don't have that charted for you today 
- We have a compelling Scientific Program

- We have innovative, new, technology that works

- IRES/IPHC has made large contributions to the Enterprise

- There are interesting challenges in putting the technology to work

- A vigorous $R \& D$ program is appropriate

- There is a good conceptual-project management plan

- The plan needs work ... but there is light at the end of the tunnel

\section{Ready for a Professional Project Engineer S STAR}

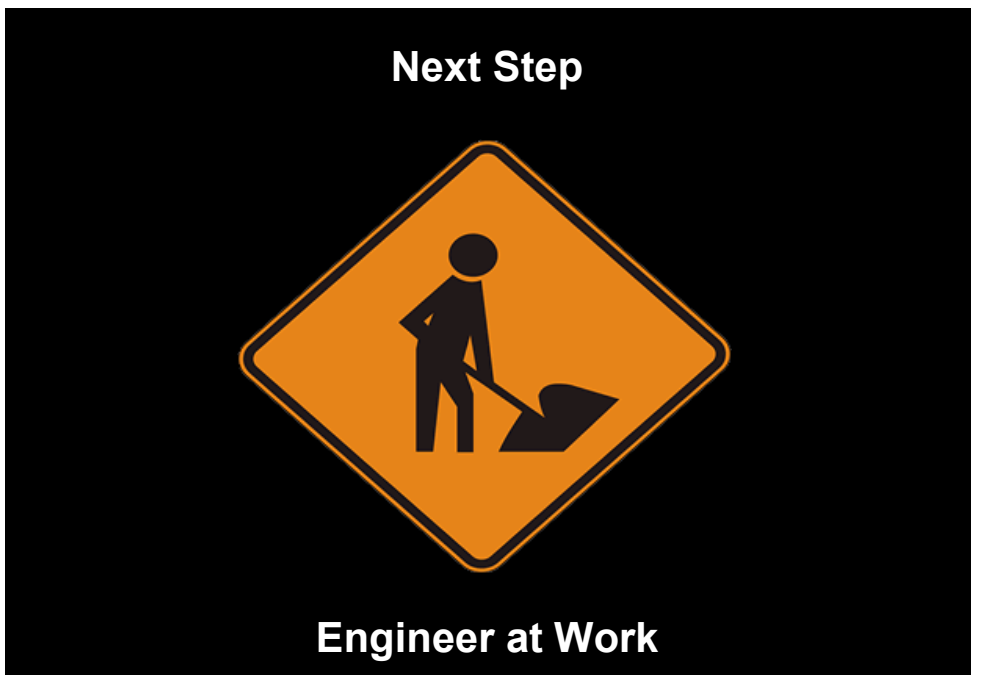




\title{
14 Appendix VI - Report of the Technical Committee
}

\author{
[ Excerpts from the report of the Technical Advisory Committee ]
}

\author{
Report of the Technical Advisory Committee for RHIC Detector Upgrades \\ March 14-16, 2006
}

\begin{abstract}
Committee members: M. Cooper (LANL), C. Haber (LBNL), B. Mecking (JLab), $J$. Proudfoot (ANL), V. Radeka (BNL), R. VanBerg (U. Penn, not present at the review), J. Va'vra (SLAC)
\end{abstract}

\section{Introduction}

This committee was convened by BNL to provide advice to the Laboratory on four proposed upgrades to the PHENIX and STAR detectors:

- PHENIX Forward Vertex Tracker (FVTX)

- PHENIX Nose Cone Calorimeter (NCC)

- STAR Heavy Flavor Tracker (HFT)

- STAR Integrated Tracking System (INT)

These upgrades play a prominent role in the future physics program for RHIC, as described in the "Mid-Term Strategic Plan for the Relativistic Heavy Ion Collider", submitted by BNL to DOE on Feb. 14, 2006. The committee was asked to assess each in the light of the proposed physics goals, and advise the Laboratory as to their feasibility and status of development, the soundness of the proposed costs and schedules, and their readiness to proceed as DOE construction projects on the proposed time scales.

The proposed upgrades are not all at the same stage of development. The two PHENIX upgrades (FVTX and NCC) are well advanced in their scientific and technical development, and are being proposed as Major Item of Equipment (MIE) projects to be funded in FY2008, with a Total Project Cost of less than \$5M each. The two STAR projects are in a more developmental stage. The HFT relies on a newly-emerging technology for silicon pixel detectors, requiring substantial R\&D effort in the years 20062008. The INT involves an optimization of the overall tracking configuration in STAR that is presently still under study. The STAR collaboration is aiming for both of these upgrades to begin construction as MIE projects in FY 2009.

The committee notes that both PHENIX and STAR have specified thin-walled beryllium beam pipes with a diameter of $3 \mathrm{~cm}$ in conjunction with the proposed vertex detectors. We recommend that this challenging aspect of the upgrade program be undertaken as a joint effort with the experts in the Collider-Accelerator Department, and with oversight by the Laboratory. 
The committee's findings and recommendations for the four proposals are given in the following sections.

\section{STAR Heavy Flavor Tracker and Integrated Tracking}

STAR has proposed a precision vertex tracking device, the Heavy Flavor Tracker, using a new technology for fine-grained pixel detectors. This uses CMOS Active Pixel Sensors (APS) technology, with the potential for $30 \mu \mathrm{m}$ pixel size on silicon sensors only $50 \mu \mathrm{m}$ thick. The technology is being developed at the IReS Laboratory in Strasbourg, France, as well as at LBNL. The proposed HFT would utilize two layers of such sensors, at $2 \mathrm{~cm}$ and $5 \mathrm{~cm}$ radii from the collision axis. The readout rate is relatively slow $(4 \mathrm{msec}$ per frame, with possible upgrade to $0.2 \mathrm{msec}$ ), resulting in multiple-event pile-up, but the occupancy is very small, as the detector will have 108 pixels. The detector would rely on additional tracking information to make the connection from TPC tracks to the correct hit segments in the pixel layers. The existing Silicon Strip Detector (SSD) may be used for an intermediate space point. However, as part of its Integrated Tracking upgrade (INT), STAR is proposing a new 3-layer set of silicon strip detectors to surround the HFT.

In addition to the intermediate layers of silicon strip detectors to surround the HFT, the Integrated Tracking upgrade aims to provide new tracking capability in the forward direction, at angles subtended by the End Cap Electromagnetic Calorimeter, in the pseudorapidity range $\eta \sim 1-2$, where the TPC tracking is not adequate to resolve the charge sign of high-energy electrons from $\mathrm{W}_{ \pm}$decays. In the present proposal, the forward tracking elements would consist of four discs of silicon strips close to the collision region, and larger-area detectors downstream using gas detectors with segmented readout based on GEM technology. The collaboration is still in the process of simulating the physics measurements and optimizing the configuration for the Integrated Tracking upgrades.

\section{Heavy Flavor Tracker}

\section{Physics Motivation}

The proposal to add a heavy-flavor tracker (HFT) to the STAR detector will significantly enhance the capabilities of STAR in the mid-rapidity range. The detection of a displaced secondary vertex will cleanly identify the production of heavy flavors from the topology of the event alone. For example, D-meson decays can be identified without the need for kaon identification and without combinatorial background.

Heavy flavor measurements are important for the RHIC program to clarify the properties of the dense medium created in heavy-ion collisions. Important issues to be addressed are the questions regarding the extent to which thermalization, flow, and energy loss for heavy quarks differ from what has been observed for the light flavors.

In addition to its role in heavy-flavor identification, the low-mass HFT will also play an important role in reducing the background in vector meson mass spectra (measured in e+e-decays) due to contributions from photon conversions. 


\section{Detector Concept and Technology}

The proposed APS technology is the most promising choice for high granularity, low radiation length, and low power dissipation vertex tracking in an environment such as RHIC.

The proposed design configuration maximizes the solid angle coverage while keeping the number of detector layers and the area of silicon at a minimum. It is assumed that the small beam pipe diameter will be consistent with reliable machine operation. The overall concept and implementation plan is well thought out.

This is a cutting edge technology, and it will be used for the first time on a fairly large scale in a large physics experiment. If successful, it will have a significant impact on future experiments.

\section{Technical Issues}

The basic sensor cell (pixel), while based on "standard" CMOS process, depends critically on some properties of the process usually not specified by the foundry. For example, the dark current at the femto-amp level is not important for most CMOS applications. While the broad-based and outstanding R\&D program at IRES is commendable, one will have to capture a good point in the continuing development progress and an available CMOS process to focus on the design of the device to be used for HFT.

The decision on how thin the sensors need to be should be based on the yield, and ease of handling of the sensors, as well as on minimizing multiple scattering, taking into account other materials necessary in the detector assembly.

The mechanical design concept makes use of advanced composite materials. The present design is mostly motivated by the desire to make possible installation and removal of the detector in a short time. Design variations which would improve the position stability of the detector components with respect to each other might be considered. A well thought out design for the readout was presented.

\section{Cost, Schedule, Manpower}

- This is a well planned project which recognizes that it has limitations in the available manpower and funding, particularly with respect to mechanical engineering.

- The overall listed manpower ( 25 FTE integrated over a five year period) will not be sufficient.

- Given the cutting edge technology, the proposed schedule does not contain sufficient float.

- The cost estimate has been made with realistic contingency figures.

- Silicon fabrication costs are not a major part of the project cost, and allowing for four detector copies is justified. 


\section{$\underline{\text { Recommendations }}$}

1. This R\&D effort should proceed. The proposed schedule for realizing a working detector is very tight, and, as the STAR project manager noted at this review, the funding profile shown in BNL's Mid-Term Plan for RHIC is not well matched to the current plan for R\&D activities. BNL and STAR should work with DOE to make realistic plans for this valuable project.

2. The STAR/HFT team should consider a third detector layer in coordination with the Intermediate Tracker, and analyze various aspects of an integrated design concept. 


\section{References}

${ }^{1}$ F. Karsch,. Nucl. Phys. A699, 199c (2002).

${ }^{2}$ K.H. Ackermann et al., Phys. Rev. Lett. 86, 402 (2001).

${ }^{3}$ J. Adams et al., Phys. Rev. Lett. 91, 172302 (2003).

${ }^{4}$ C. Adler et al., Phys. Rev. Lett. 90, 082302 (2003).

${ }^{5}$ J. Adams et al. (STAR Collaboration), Nucl. Phys. A 757, 102 (2005).

${ }^{6} \mathrm{~J}$. D. Bjorken, FERMILAB-Pub-82/59-THY.

${ }^{7}$ X. N. Wang, M. Gyulassy, I. Vitev, and B. Zhang, nucl-th/0302077.

${ }^{8}$ D. Schiff, R. Baier, and B. Zakharov, Ann. Rev. Nucl. Part. Sci., 50, 37 (2000).

${ }^{9}$ J. Ashman et al., Phys. Lett. B206, 364 (1988).

${ }^{10}$ J. Ashman et al., Nucl. Phys. B328, 1 (1989).

${ }^{11}$ B.L. Combridge, Nucl. Phys. B151, 429 (1979).

${ }^{12} \mathrm{~B}$. Mueller, nucl-th/0404015.

${ }_{14}^{13}$ R. Vogt, hep-ph/0203151.

${ }^{14}$ K.J. Eskola, V.J. Kolhinen and R. Vogt, Nucl. Phys. A696, 729 (2001).

${ }^{15}$ P. Levai, B. Mueller, and X.N. Wang, Phys. Rev. C51, 3326 (1995) : P. Levai and R. Vogt, Phys. Rev. C56, 2707 (1997).).

${ }^{16}$ K. Adcox et al., Phys. Rev. Lett. 88, 192303 (2002).

${ }^{17}$ J. Adams et al. (STAR Collaboration), Phys. Rev. Lett. 94, 062301 (2005); nucl-ex/0407006.

${ }^{18}$ T. Sjostrand et al., Computer Physics Commun. 135, 238 (2001). PYTHIA 6.152 was used with the parameter settings: MSEL $=1$, CTEQ5M1. Bottom contribution was estimated to be $20-30 \%$ in $\mathrm{p}_{\mathrm{T}}=2-3$ $\mathrm{GeV} / \mathrm{c}, 40-50 \%$ in 3-4 GeV/c to the total non-photonic electrons, and negligible at lower $\mathrm{p}_{\mathrm{T}}$.

${ }^{19}$ R. Vogt, Private Communication.

${ }^{20}$ S. Batsouli et al., Phys. Lett. B557, 26 (2003).

${ }^{21}$ X. Dong, "Open Charm Production at RHIC" QM2005, nucl-ex/0509038.

${ }^{22}$ J. Adams et al. (STAR Collaboration), Phys. Rev. Lett. 95, 122301 (2005).

${ }^{23}$ J. Adams et al. (STAR Collaboration)., Phys. Rev. Lett. 92, 052302 (2004).

${ }^{24}$ K. Schweda et al., J. Phys. G 30, S693 (2004).

${ }^{25}$ J. Castillo et al., J. Phys., G 30, S1207 (2004).

${ }^{26}$ J. Adams et al., (STAR Collaboration) Phys. Lett. B 616, 8 (2005)

${ }^{27}$ E. Shuryak, Phys. Lett. B78, 150 (1978).

${ }^{28}$ R. Rapp and J. Wambach, Adv. Nucl. Phys. 25, 1 (2000).

${ }^{29}$ R. Rapp, Phys. Rev. C 63, 054907 (2001); R. Rapp, hep-ph/0201101; R. Rapp and J. Wambach, hep$\mathrm{ph} / 9909229$.

${ }^{30}$ U. Heinz and P.F. Kolb, Nucl. Phys. A702, 269c (2002).

${ }^{31}$ H. Sorge, Phys. Rev. Lett. 78, 239 (1997); H. Sorge, Phys. Rev. Lett. 82, 2048 (1999).

${ }^{32}$ C. Adler et al., (STAR Collaboration), Phys. Rev. Lett. 87, 262301 (2001).

${ }^{33}$ C. Adler et al., (STAR Collaboration), Phys. Rev. Lett. 89, 132301 (2002).

${ }^{34}$ P. Huovinen et al., Phys. Lett. B503, 58 (2001).

${ }^{35}$ M. Kaneta, J. Phys. G: Nucl. Part. Phys. 30, S1217 (2004).

${ }^{36}$ V. Greco, C.M. Ko, and R. Rapp, Phys. Lett. B595, 202 (2004)

${ }^{37}$ J. Stachel, Nucl. Phys. A654, 119c (1999).

${ }^{38}$ P. Braun-Munzinger, D. Magestro, K. Redlich, and J. Stachel, Phys. Lett. B518, 41 (2001).

${ }^{39}$ P. Braun-Munzinger, K. Redlich, and J. Stachel, nucl-th/0304013.

${ }^{40}$ E. Schnedermann, J. Sollfrank, and U. Heinz, Phys. Rev. C48, 2462 (1993).

${ }^{41}$ P. Braun-Munzinger and J. Stachel, Phys. Lett. B490, 196 (2000).

${ }^{42}$ P. Braun-Munzinger and J. Stachel, Nucl. Phys. A690, 119c (2001).

${ }^{43}$ A. Andronic, P. Braun-Munzinger, K. Redlich, and J. Stachel,. Phys. Lett. B571, 36 (2003).

${ }^{44} \mathrm{http}: / / \mathrm{www}-\mathrm{h} 1 . d e s y . d e / p s f i l e s / c o n f p a p / E P S 2003 / H 1$ prelim-02-076.ps

$45 \mathrm{http}: / / \mathrm{www}-$ zeus.desy.de/physics/phch/conf/eps03/564/paper.pdf

${ }^{46}$ T.Sjöstrand, Comp. Phys. Commun. 82, 74 (1994). 
${ }^{47}$ A. Andronic et al., Phys. Lett. B571, 36 (2003).

${ }^{48}$ M. Djordjevic, M. Gyulassy, and S. Wicks, Phys. Rev. Lett. 94, 112301(2005).

${ }^{49}$ Y.L. Dokshitzer and D.E. Kharzeev, Phys. Lett. B519, 199(2001).

${ }^{50}$ N. Armesto, A. Dainese, C.A. Salgado, U. Wiedemann, Phys. Rev. D71, 054027(2005).

${ }^{51}$ S. S. Adler et al. (STAR Collaboration),., Phys. Rev. Lett. 91, 072301 (2003).

${ }^{52}$ S. S. Adler et al. (STAR Collaboration)., Phys. Rev. Lett. 91, 241803 (2003).

${ }^{53}$ C. Adler et al. (STAR Collaboration)Phys. Rev.., Lett. 90, 082302 (2003).

${ }^{54}$ M. Gyulassy and M. Plümer, Nucl. Phys. A527, 641c (1991).

${ }^{55}$ Y. L. Dokshitzer and D. E. Kharzeev, Phys. Lett. B519, 199 (2001).

${ }^{56} \mathrm{H}$. Buesching, (Phenix Collaboration), QM05 proceedings.

${ }^{57}$ J. Dunlop, (STAR Collaboration), QM05 proceedings.

${ }^{58} \mathrm{X}$. Dong, charm production overview, QM05 proceedings.

${ }^{59}$ J. Adams, et al. (STAR Collaboration), Phys. Rev. Lett. 91 (2003) 072304.

${ }^{60}$ S.S. Adler et al. (Phenix Collaboration), Phys. Rev. Lett. 91, 072301 (2003).

${ }^{61}$ M. Djordjevic, M. Gyulassy, R. Vogt, and S. Wicks, nucl-th/0507019.

${ }^{62}$ H. van Hees, V. Greco, and R. Rapp, nucl-th/0508055.

${ }^{63}$ K. Abe, et al. (Belle Collaboration), Phys. Rev. Lett. 89, 142001(2002).

${ }^{64}$ S. Baek, P. Ko, J. Lee, and H.S. Song, J. Korean Phys. Soc. 33, 97 (1998).

${ }^{65}$ P.L.Cho and A.K. Leibovich, Phys. Rev. D54, 6690(1996).

${ }^{66}$ F. Yuan, D.F. Aiao, and K.T. Chao, Phys. Rev. D56, 321(1997).

${ }^{67}$ B.L. Ioffe and D.E. Kharzeev, Phys. Rev. D69, 014016(2004).

${ }^{68}$ M. Djordjevic, M. Gyulassy, R. Vogt, S. Wicks, nucl-th/0507019.

${ }^{69}$ D.E. Kharzeev and K. Tuchin, Nucl. Phys. A753, 316(2005).

${ }^{70}$ R. Rapp, Phys. Rev. C63, 054907 (2001).

${ }^{71}$ X. Dong, P. Sorensen, N. Xu, in preparation, Sept. 2005.

${ }^{72}$ A. Marín et al., J. Phys. G: Nucl. Part. Phys. 30, S709 (2004).

${ }^{73}$ D. Adamova et al. (CERES Collaboration), Phys. Rev. Lett. 91 (2003) 042301.

74 R. Rapp, Phys. Rev. C63, 054907 (2001).

${ }^{75}$ R. Rapp, Pramana 60, 675 (2003).

${ }^{76}$ G. Bunce, N. Saito, J. Soffer, and W. Vogelsang, Annu. Rev. Nucl. Part. Sci. 49, 303 (1999).

${ }^{77}$ M. Karliner and R.W. Robinett, Phys. Lett. B 324, 209 (1994).

${ }^{78}$ I. Bojak and M. Stratmann Phys. Rev. D 67, 034010 (2003).

${ }^{79}$ M. Gluck, E. Reya, M. Stratmann, and W. Vogelsang Phys. Rev. D 63, 094005 (2001).

${ }^{80}$ K.H. Ackerman et al., Nucl. Instr. and Meth. A 499, 624 (2003).

${ }^{81}$ ALICE Technical Design Report of the Inner Tracking System, CERN/LHCC 99-12, ALICE TDR 4 (1999).

${ }^{82}$ L. Ray and R.S. Longacre, nucl-ex/0008009 (2000).

${ }^{83}$ GEANT -- Dectector Description and Simulation Tool, The CERN computing center. CERN Program Library Long Writeup W5013, and Technical Report: http://www.star.bnl.gov/STAR/comp/simu/newsite/gstar/Manual/manual_index.html

${ }^{84}$ L. Arnold, J. Baudot, D. Bonnet, et. al., "The STAR silicon strip detector (SSD)", Nucl. Instr.. and Meth., A 499, 652,(2003).

${ }^{85}$ Particle Data Group, Phys. Lett. B., Volume 592, Issues 1-4, 15 July 2004, p245.

${ }^{86}$ Karimäki, Nucl. Instrum. Methods A410, 284 (1998).

${ }^{87} \mathrm{http}: / / w w w . s t a r . b n l . g o v / p r o t e c t e d /$ spectra/macross/glauber/flow/FlowGlauberCalculations.htm

${ }^{88} \mathrm{~W}$. Fischer, RHIC-II workshop, http://www.bnl.gov/panic05\%5Fsatellite/

${ }^{89}$ Particle Data Book, Phys. Lett. B592, 1 (2004).

${ }^{90}$ Technical report, http://www-rnc.lbl.gov/ wieman/HitDensityMeasuredLuminosity.htm

${ }^{91}$ Technical report, http://www-rnc.lbl.gov/ wieman/TrackDensityApr2004.htm

92 Jim Thomas, Private Communication.

${ }^{93}$ K. Abe, A. Arodzero, C. Baltay, J.E. Brau, M. Breidenbach, P.N. Burrows et al., "Design and performance of the SLD vertex detector: a 307 Mpixel tracking system," Nucl. Instr. and Meth., A 400, 287 (1997).

${ }^{94}$ J. Janesick, Scientific Charge-Coupled Devices, SPIE Press, Bellingham, WA, 2001. 
${ }^{95}$ K.D. Stefanov, “CCd-based vertex detector for the future linear collider”, Nucl. Instru. and Meth. A 549, 93 (2005).

${ }^{96}$ C.J.S. Damerell, "Charge coupled devices as particle tracking detectors," Rev. Sci. Instr., vol. 69, pp. 1549-1573, 1998.

${ }^{97}$ J. Kemmer and G. Lutz, Nucl. Instr. and Meth. A 253, 365 (1987).

${ }^{98}$ G. Lutz, "DEPFET development at the MPI semiconductor laboratory", Nucl. Instr. and Meth. A 549, $103,(2005)$.

${ }^{99} \mathrm{http} / / /$ ireswww.in2p3.fr/ires/recherche/capteurs/

${ }^{100}$ E. R. Fossum, "CMOS Image Sensors: Electronic Camera-On-A-Chip", IEEE Trans. On Electron Devices, 44, 1689 (1977).

${ }^{101}$ R. Turchetta et al., Nucl. Instr. and Meth. A 458, 677 (2001).

${ }^{102}$ M. Winter et al., Proc. of the 8th ICATPP, Como Italy (2003).

${ }^{103}$ MIMOSA: Standing for Minimum Ionizing particle MOS Active pixel sensor.

${ }^{104}$ M. Devaux et al., Nucl. Instr. and Meth. A 512, 71 (2003).

${ }^{105}$ W. Dulinski et al., Proc. IEEE Nuclear Science Symposium, Portland OR. Trans. Nucl. Sci. (2003).

${ }^{106} \mathrm{http}: / /$ www.lbnl.leog.org/thinned_mimosa_testing.htm

${ }^{107}$ Technical report, http://ireswww.in2p3.fr/ires/recherche/capteurs/index.html

${ }^{108}$ H. S. Matis, F. Bieser, S. Kleinfelder, G. Rai, F. Retiere, H.G. Ritter, K. Singh, S.E. Wurzel, H.H. Wieman, and E. Yamamoto, IEEE Trans. on Nucl. Sci. 50, 1020 (2003).

${ }^{109}$ S. Kleinfelder, H. Bichsel, F. Bieser, H. S. Matis, G. Rai, F. Retiere, H. Weiman, and E. Yamamoto, Proceedings of the SPIE, Hard X-Ray and Gamma Ray Detector Physics IV, July 2002, Vol. 4784, pp 208.

${ }^{110}$ H.S. Matis, F. Bieser, Y. Chen, R. Gareus, S. Kleinfelder, M. Oldenburg, F. Retiere, H.G. Ritter, H.H. Wieman, S.E. Wurzel, E Yamamoto, Nucl. Instr. and Meth. A549, 130 (2005).

${ }^{111}$ S. Kleinfelder, S. Li, F. Bieser, R. Gareus, L. Greiner, J. King, J. Levesque, H.S. Matis*, M. Oldenbur, H.G. Ritter, F. Retiere, A. Rose, K. Schweda, A. Shabetai, E. Sichtermann, J.H. Thomas, H.H. Wieman, H. Bichsel, LBNL 59121. Submitted to the Proceedings of Pixel 2005, Bonn, Germany (2005).

${ }^{112}$ S. Kleinfelder, F. Bieser, Y. Chen, R. Gareus, H.S. Matis, M. Oldenburg, F. Retiere, H.G. Ritter, H.H. Wieman, E. Yamamoto, IEEE Trans. Nucl. Sci. 51, 2328 (2004).

${ }^{113}$ N.H. Xuong, A.-C. Milazzo, P. LeBlanc, F. Duttweiler, J. Bouwer, S. Peltier, M. Ellisman, P. Denes, F. Bieser, H.S. Matis, H. Wieman, S. Kleinfelder, In "Sensors and Camera Systems for Scientific, Industrial, and Digital Photography Applications V", SPIE 5301 242, (2004).

${ }^{114} \mathrm{Technical}$ report, http://www-lepsi.in2p3.fr/ huch/MimoStar181203.pdf

115 Technical report, http://www-rnc.lbl.gov/ wieman/RadiationLengthsProposal.htm

$116 \mathrm{http}: / /$ www-rnc.lbl.gov/ wieman/radiationdoseinapsDulinskiValue.htm

$117 \mathrm{http}: / /$ doc.cern.ch/yellowrep/2001/2001-006/p1.pdf

$118 \mathrm{http} / / / \mathrm{www}$. lbnl.leog.org/writeup.htm

119 Technical report, http://www-rnc.lbl.gov/ wieman/StiffnessLadder1.htm

${ }^{120}$ TV Holography System at LBNL, courtesy of Eric Andersen and the ATLAS project.

${ }^{121}$ Technical report, $h \mathrm{ttp}: / /$ www.lionprecision.com/modular/probes.html

${ }^{122}$ Technical report, http://www.lbnl.leog.org/writeup.htm

${ }^{123}$ Technical report, http://www.lbnl.leog.org/ir prelim writeup.htm

${ }^{124}$ Technical report, http://www.lbnl.leog.org/cable constraints.htm

${ }^{125}$ Technical report, http://www.lbnl.leog.org/crossing lines measurement first look.htm

${ }^{126}$ Steve Peggs and Steve Tepikian, Private Communication.

${ }^{127} \mathrm{~S}$. Tepikian,. Twiss functions and beam sizes of the RHIC intersection. RHIC/AP25 (1994).

${ }^{128}$ Technical report, http://www-rnc.lbl.gov// wieman/BeamPipeRadiusLimitsRHICAP25.htm

${ }^{129}$ Technical report, http://www-rnc.lbl.gov/ wieman/WolframFisherApertureNote.htm

${ }^{130}$ Technical report, http://www-rnc.lbl.gov/ wieman/BeamPipeVacuumShortAns.htm

${ }^{131}$ John O'Hanlon, A User's Guide to Vacuum Technology. John Wiley and Sons, second edition (1989).

${ }^{132}$ Seth Wieman, Private Communication.

${ }^{133}$ Technical report, http://www-rnc.lbl.gov/ wieman/TopThinPipeAnalysis.htm

${ }^{134}$ Sandro Bravar, Private Communication.

${ }^{135}$ Dan Weiss, Private Communication 30-Nov-2004. 
136 "Mid-Term Strategic Plan: 2006-2011 for the Relativistic Heavy Ion Collider at Brookhaven National Laboratory", Prepared by Brookhaven National Laboratory with the RHIC Scientific Community for the U.S. Department of Energy February 14, 2006. 